\title{
A Methodology to Assess the Value of Integrated Hydropower and Wind Generation \\ by
}

\author{
Mitch A. Clement \\ B.A., Wheaton College, 2000 \\ B.S., University of Colorado, 2010
}

\author{
A thesis submitted to the \\ Faculty of the Graduate School of the \\ University of Colorado in partial fulfillment \\ of the requirements for the degree of \\ Master of Science \\ Department of Civil, Environmental and Architectural Engineering


This thesis entitled:

A Methodology to Assess the Value of Integrated Hydropower and Wind Generation written by Mitch A. Clement

has been approved for the Department of Civil, Environmental and Architectural Engineering

Edith Zagona

Balaji Rajagopalan

JoAnn Silverstein

Date

The final copy of this thesis has been examined by the signatories, and we find that both the content and the form meet acceptable presentation standards of scholarly work in the above mentioned discipline. 
Clement, Mitch A. (M.S. Civil Engineering)

A Methodology to Assess the Value of Integrated Hydropower and Wind Generation Thesis directed by Professor Edith Zagona

Installed wind generation capacity has increased at a rapid rate in recent years. Wind generation provides numerous economic, social and environmental benefits, but it also carries inherent variability and uncertainty, which can increase the need for additional balancing reserves, generation resources that can adjust their output rapidly to keep power supply in balance with demand. Hydropower is an inexpensive and flexible generating resource that has been considered one of the best resources to provide the necessary balancing reserves for wind. Hydropower's flexibility and capacity are limited, however, by non-power constraints associated with environmental and water management objectives that have not been fully accounted for in previous wind integration studies. We present a methodology to evaluate hydropower and wind integration using the RiverWare river system and hydropower modeling tool. The model represents both the physical characteristics of the hydropower system and accounts for realistic non-power policy constraints. An economic evaluation is provided that includes the value of both energy and ancillary services. In addition, operational outputs include the ability to satisfy all policy constraints. The methodology is applied to a test case integrated hydropower and wind generation system including five hydropower projects in a run-of-river configuration for a range of wind penetration levels and hydrologic conditions.

Results show that wind at low penetrations adds economic value to the system. As the installed capacity increases, additional wind generation has diminishing returns, primarily due to increased reserve requirements. Increased wind capacity also causes increases the number of policy constraint violations. Non-power constraints have a significant impact on total system value, but that relative impact varies depending on system conditions. Complex interactions between policy and the physical system result in a highly non-linear response of the system to changes in wind penetration. Utilization of goal programming makes it possible to capture these effects that would 
be missed without a realistic representation of both the integrated physical system and its operating policy. This methodology can be used to provide an improved representation of hydropower systems in future wind integration studies. 


\section{Acknowledgements}

I would like to thank my advisor, Edie Zagona, for providing this research opportunity. This project would not have been possible without her guidance throughout the process.

Tim Magee at CADSWES provided invaluable support throughout this project both on technical matters and in analyzing the broader implications of the work. I am grateful to benefit from his experience and expertise. I would also like to thank my other committee members, Balaji Rajagopalan and JoAnn Silverstein for their time and valuable input on this thesis.

I am very grateful to the Hydro Research Foundation and the Department of Energy for providing me with a fellowship to fund this research.

Joe Taylor from Mid-Columbia Central is responsible for providing Mid-C request data used in this research. In addition, he provided insight into Mid-C operations and policy that influenced this work and facilitated the modeling of realistic operating conditions.

Much of the groundwork for this project was laid by a previous project sponsored by Oak Ridge National Laboratory to model wind integration in the Mid-Columbia system. I thank Brennan Smith of ORNL for his contribution and guidance through that project.

Bri-Mathias Hodge and Michael Milligan of the National Renewable Energy Laboratory provided insight on wind modeling and facilitated the acquisition of wind forecast data that made possible the development of the wind forecast model in this research.

Finally, I would like to thank my wife, Katie, and our daughters, Anna, Josie and Ruthie, for providing endless support, encouragement and patience throughout this process. 


\section{Contents}

\section{Chapter}

1 Introduction 1

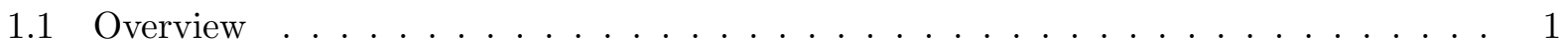

1.2 Existing Work on Hydropower and Wind Integration . . . . . . . . . . . . . 5

1.3 Significance of this Research . . . . . . . . . . . . . . . . . . . 10

2 Modeling and Methodology 15

2.1 Conceptual Model . . . . . . . . . . . . . . . . . . . . . . . 15

2.1 .1 Model Overview . . . . . . . . . . . . . . . . . 15

2.1 .2 Ancillary Services . . . . . . . . . . . . . . . . . . 19

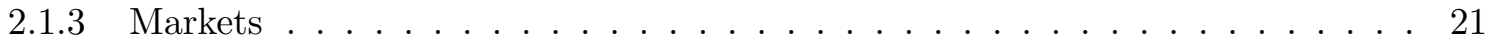

2.2 Run Sequence . . . . . . . . . . . . . . . . . . . . . . 23

2.3 Hydropower Modeling in RiverWare . . . . . . . . . . . . . . . . . 28

2.3.1 RiverWare Optimization - Preemptive Linear Goal Programming . . . . . . . 28

2.3.2 Power Modeling . . . . . . . . . . . . . . . . 30

2.3 .3 Ancillary Services . . . . . . . . . . . . . . . . . . . . 30

2.3.4 Economic Modeling . . . . . . . . . . . . . . . . . . . 34

2.3.5 Other Physical Processes _. . . . . . . . . . . . . . . . . 39

2.4 Test System . . . . . . . . . . . . . . . . . . . . . 40

2.4 .1 Physical System Description . . . . . . . . . . . . . . . . 40 


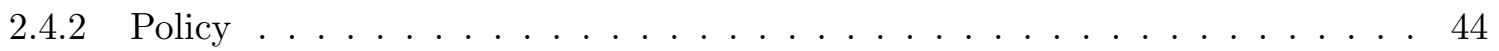

2.4 .3 Input Data . . . . . . . . . . . . . . . . . . . 52

2.4 .4 Scenario Descriptions . . . . . . . . . . . . . . . . . 62

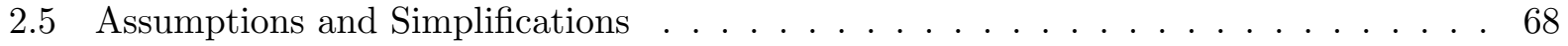

3 Wind Generation Modeling $\quad 70$

3.1 Actual Wind Generation Data . . . . . . . . . . . . . . . . 71

3.2 Wind Generation Forecast . . . . . . . . . . . . . . . . . . . . 74

3.2.1 Existing Statistical Wind Forecast Methodologies . . . . . . . . . . . . . . . 74

3.2.2 Analysis of Observed Wind Generation Forecast Error . . . . . . . . . . . . 75

3.2.3 Day-ahead Wind Forecast Model . . . . . . . . . . . . . . . . . 78

3.2.4 Wind Forecast Models for Two and Three Days Ahead . . . . . . . . . . . . 83

4 Assessing the Value of Integrated Hydropower and Wind Generation 86

4.1 Introduction $\ldots \ldots \ldots \ldots \ldots \ldots$

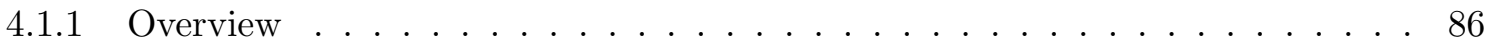

4.1.2 Existing Wind and Hydropower Integration Studies _ . . . . . . . . . . 87

4.1.3 Significance of this Research . . . . . . . . . . . . . . . . 88

4.2 Modeling and Methodology . . . . . . . . . . . . . . . . 89

4.2 .1 Conceptual Model . . . . . . . . . . . . . . . . . . . . 89

4.2.2 Hydropower Modeling in RiverWare . . . . . . . . . . . . . . . . . . . . 90

4.2 .3 Run Sequence . . . . . . . . . . . . . . . . . . . . 93

4.3 Wind Modeling . . . . . . . . . . . . . . . . . . . . . 94

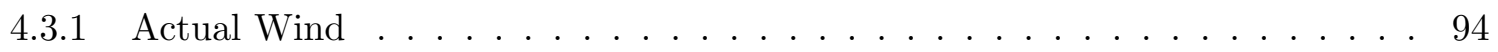

4.3 .2 Wind Forecast . . . . . . . . . . . . . . 96

4.4 Test Case . . . . . . . . . . . . . . . . . . . . . . . . 99

4.4 Physical Hydropower System . . . . . . . . . . . . . . . . . . 99

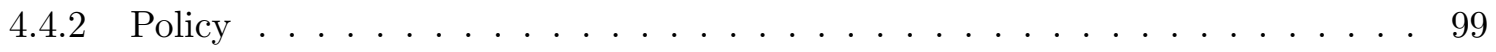




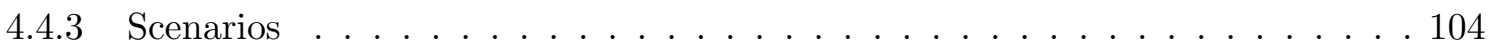

4.4 .4 Input Data . . . . . . . . . . . . . . . . . . 106

4.5 Discussion of Results . . . . . . . . . . . . . . . . . . . . . 107

4.5.1 Impact of Non-power Policy . . . . . . . . . . . . . . . . . . . 107

4.5.2 Isolated Effects of Wind Forecast Error, Variability and Energy . . . . . . . . 116

4.5.3 Sensitivities to System Characteristics . . . . . . . . . . . . . . 117

4.6 Conclusions . . . . . . . . . . . . . . . . . . . . . . 122

5 Conclusions and Recommendations 124

5.1 Summary . . . . . . . . . . . . . . . . . . . . . . 124

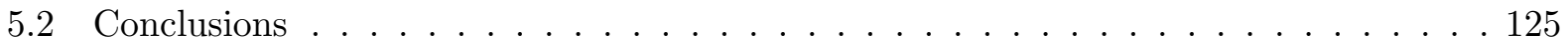

5.3 Future Work . . . . . . . . . . . . . . . . . . . . 129

$\begin{array}{ll}\text { Bibliography } & 133\end{array}$

\section{Appendix}

A Plant Power Tables 138

B Wind Modeling Supplemental Analysis $\quad 144$

B.1 Wind Variability Analysis . . . . . . . . . . . . . . . . . . . . . . 144

B.2 Wind Forecast Model Analysis . . . . . . . . . . . . . . . . . . . . 145

$\begin{array}{llr}\text { C Additional Model Results and Analysis } & 148\end{array}$

C.1 Policy Effects on System Economic Value . . . . . . . . . . . . . . . . 148

C.2 Impacts of Flow Constraints on Upstream Reservoir Operations . . . . . . . . . . . 151

C.3 Constraint Violations . . . . . . . . . . . . . . . . . . . . . . . . 153

C.4 Effects of Wind Forecast Error, Variability and Energy . . . . . . . . . . . . 155

C.5 Effects of Reserve Requirements . . . . . . . . . . . . . . . . . 161 
C.6 Summer Scenario with Spring Prices . . . . . . . . . . . . . . 163

C.7 Effects of Load Levels and Transmission Limits . . . . . . . . . . . . . . . . . . . 165

C.8 Market Depth Effects . . . . . . . . . . . . . . . . . . . . . . 168

C.9 Effects of Wind Timing and Correlation with Prices _ . . . . . . . . . 168 


\section{Tables}

\section{Table}

2.1 Reservoir storage and elevation-volume parameters . . . . . . . . . . . . . . 42

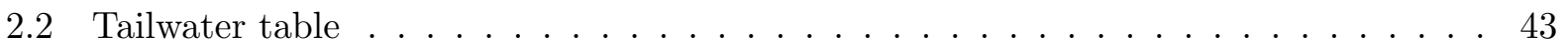

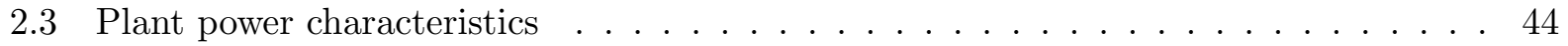

2.4 Load input data for base scenarios . . . . . . . . . . . . . . . . 53

2.5 Load forecast model parameters . . . . . . . . . . . . . . . . 54

2.6 Incremental reserve requirments, spring base scenario . . . . . . . . . . . . . 61

2.7 Load input data for alternative load level scenarios . . . . . . . . . . . . . 67

3.1 Statistics of normalized observed wind generation forecast error over a range of lead

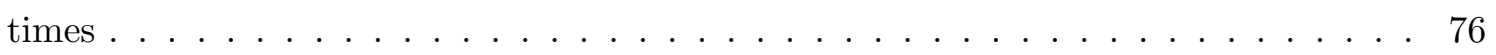

3.2 ARMA model fitting AIC values . . . . . . . . . . . . . . . 80

3.3 Wind forecast error model Box-Cox and $\operatorname{ARMA}(2,1)$ parameter values . . . . . . 80

3.4 Statistics of observed and model forecast percent error distributions . . . . . . . . 82

3.5 Parameter values for additional error models for two day ahead and three day ahead wind generation forecasts $\ldots \ldots \ldots \ldots \ldots$

3.6 Statistics of observed and model 48 hour and 72 hour wind generation forecast error 85

4.1 Wind forecast error model Box-Cox and $\operatorname{ARMA}(2,1)$ parameter values . . . . . . . 98

4.2 Statistics of observed and model forecast error distributions . . . . . . . . . . . 98 
4.3 Correlation of hydro generation with day-ahead energy prices for various wind timing

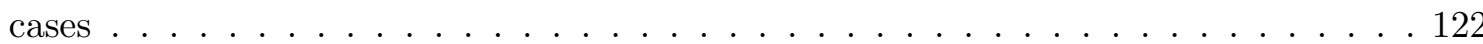

A.1 Plant power table for Reservoir $1 \mathrm{~B} \ldots \ldots \ldots \ldots$

A.2 Plant power table for Reservoir $1 \mathrm{C} \ldots \ldots \ldots$. . . . . . . . . . . . . . . . . . .

A.3 Plant power table for Reservoir 1D . . . . . . . . . . . . . . . . . . . . 141

A.4 Plant power table for Reservoir $1 \mathrm{E} \ldots \ldots$. . . . . . . . . . . . . . . . 142

A.5 Plant power table for Reservoir $1 \mathrm{~F} \ldots \ldots \ldots$. . . . . . . . . . . . . . . . . . . .

B.1 Site ID numbers for WWSIS sites used for wind variability analysis to scale wind data variability . . . . . . . . . . . . . . . . . . . . . 144

B.2 10-minute wind generation data statistics for BPA observed data from 2010 and selected $W W S I S$ sites from $2006 \ldots \ldots \ldots \ldots \ldots$

B.3 Hourly wind generation data statistics for selected WWSIS sites (2006) and BPA

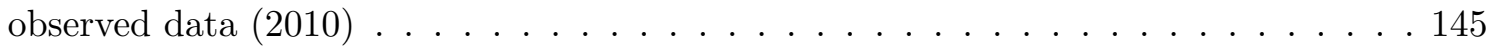

C.1 Standard deviation of total system value across multiple traces for each hydrologic

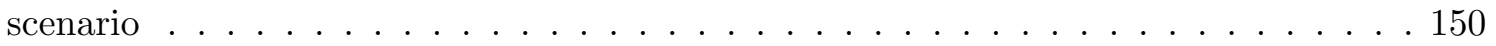




\section{Figures}

\section{Figure}

2.1 Conceptual Organizational Model: Hybrid of a vertically integrated utility and formal markets . . . . . . . . . . . . . . . . . . . . . 17

2.2 Diagram of the run sequence $\ldots \ldots \ldots \ldots \ldots \ldots \ldots$

2.3 Diagram of the two-stage optimization sequence $\ldots \ldots \ldots \ldots$

2.4 RiverWare model workspace showing 5 hydropower projects in a cascading reservoir

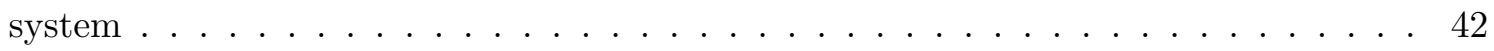

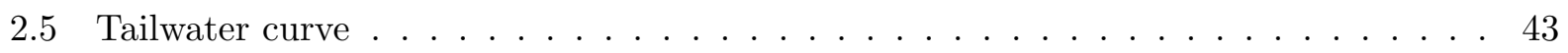

2.6 Sample energy value curve and price curve . . . . . . . . . . . . 57

2.7 Time series of actual wind and the No Variability wind for the same scenario at $30 \%$

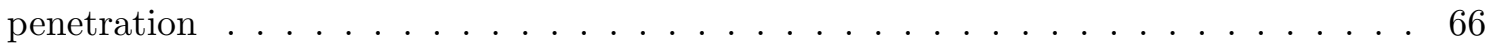

3.1 Histograms of observed wind generation forecast error at a range of lead times $\quad \ldots \quad$. 77

3.2 Histograms and QQ-Normal plots of observed wind forecast error and Box-Cox trans-

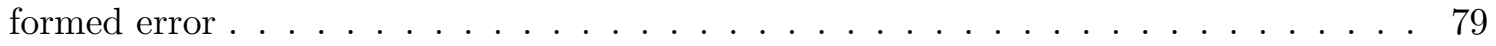

3.3 Histograms of observed wind generation forecast error and 100 traces of modeled

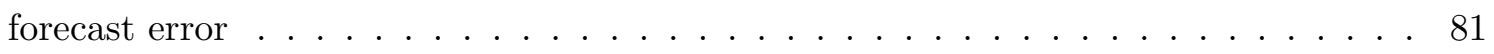

3.4 Sample trace of model forecast wind generation and actual generation $\ldots$. . . . . 83

3.5 Histograms of observed and model 48 hour and 72 hour wind generation forecast error 84

4.1 Histograms of observed and model wind generation forecast error . . . . . . . . . . 98 
4.2 RiverWare model workspace showing 5 hydropower projects in a cascading reservoir

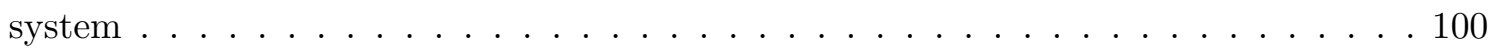

4.3 Total system value for full policy and simplified policy cases, 3 scenarios . . . . . . 109

4.4 Components of the total system value for the spring and fall base scenarios . . . . 113

4.5 Power-related constraint violations . . . . . . . . . . . . . . . . . . 114

4.6 Non-power constraint violations . . . . . . . . . . . . . . . . . . . 115

4.7 Reservoir $1 \mathrm{~B}$ spill and wind forecast error $\ldots \ldots \ldots \ldots$

4.8 Effects of isolated wind energy, variability and forecast error, spring scenario _ . . 117

4.9 Real-time market impacts on total system value and spill . . . . . . . . . . . 119

4.10 Comparison of different load following requirements for spring scenario . . . . . . 121

B.1 Scatter plots of observed wind forecasts vs. actual wind and forecast error vs. actual

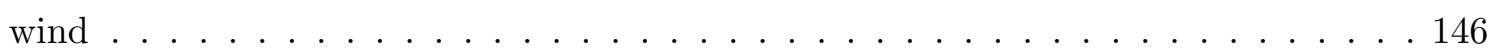

B.2 Histograms of observed and modeled wind forecast percent error . . . . . . . . . 147

C.1 Variance in total system value with full and reduced policy, spring scenario . . . . 149

C.2 Variance in total system value with full and reduced policy, summer scenario . . . 149

C.3 Variance in total system value with full and reduced policy, fall scenario . . . . . . 150

C.4 Time series plot illustrating flow requirements at Reservoir $1 \mathrm{~F}$ forcing operations upstream in the system . . . . . . . . . . . . . . . . . 152

C.5 Summary of constraint violations for base scenarios . . . . . . . . . . . . . 154

C.6 Unmet load violations for base scenarios . . . . . . . . . . . . . . . . . 154

C.7 Spring scenario effects of wind forecast error, variability and energy . . . . . . 156

C.8 Results for No Wind Forecast Error cases with full and reduced load following re-

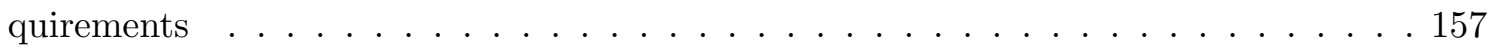

C.9 Constraint violations for No Wind Forecast Error cases with full and reduced load

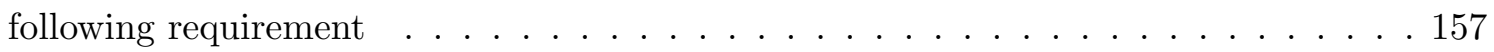

C.10 Summer scenario effects of wind forecast error, variability and energy . . . . . . . 159 
C.11 Fall scenario effects of wind forecast error, variability and energy . . . . . . . . 160

C.12 Load following reserve requirement and average wind generation vs. installed wind

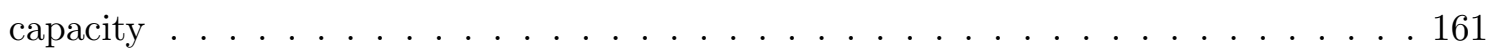

C.13 Senstivity of system total value to three levels of contingency reserve requirements . 162

C.14 Results from the summer scenario with spring energy prices . . . . . . . . . . 164

C.15 Load level effects for the spring scenario . . . . . . . . . . . . . . 165

C.16 Transmission and load combinations for the spring and summer scenarios $\ldots 167$

C.17 Market depth effects on total value, hydro generation and ancillary services . . . . 168

C.18 Effects of shifting wind timing on total system value and total hydro generation . . . 169 


\section{Chapter 1}

\section{Introduction}

\section{$1.1 \quad$ Overview}

Installed wind generation capacity has increased at a rapid rate in recent years. In 2008 the total global installed capacity was over 120,000 MW, more than double the installed capacity in 2005. ${ }^{1}$ The U.S. has just under 50,000 MW of installed wind capacity as of the second quarter of 2012. ${ }^{2}$ In the Pacific Northwest, the Bonneville Power Administration (BPA) Balancing Authority Area has over 4700 MW of wind generation capacity online as of May 2012 and plans to have over $6000 \mathrm{MW}$ by 2013. This is an increase from less than $800 \mathrm{MW}$ in $2006 .^{3}$ Much of this increase in wind generating capacity is being motivated by renewable portfolio standards (RPS) now in place in 37 states. $^{4}$ California's RPS, for example, requires $33 \%$ of all generation for investor owned utilities and electric service providers to come from renewable resources by $2020 .^{5}$

Wind is generally considered a non-dispatchable resource. ${ }^{6}$ It cannot be scheduled in the same manner as other generating resources to follow load ${ }^{7}$ or energy market patterns. The dependency of wind generation on weather conditions causes it to be inherently variable and uncertain in nature. The variability here refers to the fluctuations in wind generation, the ramping up and down, at

\footnotetext{
${ }^{1}$ http://www.gwec.net/index.php?id=13

${ }^{2}$ http://www.awea.org/learnabout/industry_stats/index.cfm

36000 MW would represent approximately $30 \%$ of BPA peak generation. http://www.bpa.gov/corporate/WindPower/index.cfm; http://www.bpa.gov/corporate/about_BPA/Facts/FactDocs/ BPA_Facts_2011.pdf

${ }^{4}$ http://www.dsireusa.org/summarytables/rrpre.cfm, last accessed September 8, 2012

${ }^{5}$ http://www.cpuc.ca.gov/PUC/energy/Renewables/overview

6 The Midwest ISO (MISO) market does include a Dispatchable Intermittent Resource product where wind resources can get paid to reduce output to mitigate oversupply (EPRI, 2011).

${ }^{7}$ In the context of power systems, load refers to energy demand.
} 
various time scales that are independent of load fluctuations. The uncertainty comes from imperfect wind forecasts.

In order to maintain stability in the electric grid, energy supply and demand must remain equal at all times within a balancing authority area (BA). ${ }^{8}$ There is no energy storage within the power grid, so supply and demand must continually be balanced on a moment-by-moment basis. This requires sufficient balancing reserves in the system, generating resources that can increase or decrease their output rapidly to keep power supply in balance with demand. ${ }^{9}$

System load also has inherent variability and uncertainty, and system operators are accustomed to dealing with this variability and uncertainty in scheduling and operating generating resources. Wind and load variability are generally uncorrelated, so at low levels of wind penetration $^{10}$ wind variability has relatively little impact on net load ${ }^{11}$ variability. The same is true for forecast error. However, on a per-unit basis, the variability and forecast error associated with wind have a larger magnitude than that of load. As wind penetration increases, therefore, net load variability and uncertainty can have a much larger magnitude, increasing the importance of maintaining sufficient flexible generating resources which can ramp up or down quickly to maintain the balance between power supply and demand. (Milligan et al., 2009)

Hydropower has often been considered one of the best balancing resources to couple with wind generation. Hydropower generators have high ramp rates. Combining this capability with automatic generation control (AGC) enables hydropower resources to respond quickly to fluctuations in system supply and demand. A hydro generator can go from zero to maximum generating capacity in a matter of minutes, a much shorter time frame than other resources. It can typically gener-

\footnotetext{
8 The North American Electric Reliability Corporation (NERC) defines a Balancing Authority Area as The collection of generation, transmission, and loads within the metered boundaries of the Balancing Authority. The Balancing Authority is the entity that must assure that the balance between electricity supply and demand is maintained at all times (http://www.nerc.com/files/Glossary_12Feb08.pdf). NERC sets the reliability standards for BAs based Area Control Error (ACE), the MW imbalance between supply and demand at each point in time. See NERC, 2007.

${ }^{9}$ In some cases major load centers are utilized as balancing reserves as well by increasing or decreasing their power demand. The use of load as a balancing resource is not explored in the research we present here.

${ }^{10}$ Wind penetration is generally defined as the percentage of wind generating capacity in a system. The specifics of how this quantity is calculated vary with different systems. Section 3.1 addresses how this quantity is defined for our study.

${ }^{11}$ Net load is total system load less wind generation.
} 
ate at reduced capacity with less efficiency loss than thermal generating resources. Hydropower generation has a low marginal cost, and the fuel supply for hydropower generation, water, is a renewable resource. Most conventional hydropower facilities also have the capability to store energy in the form of impounded water. This allows for a certain level of control over the timing of hydro generation. The amount of storage capacity available varies, however, depending on the facility's physical characteristics as well as its operational policy. ${ }^{12}$ (de Almeida et al., 2005; Acker, 2011a) Pumped storage hydropower facilities (PSH) provide the added benefit of being able to use excess energy to pump water for storage in an upper reservoir to be used later for generation in times of increased energy demand or higher energy prices thus providing additional balancing reserves for systems with variable generation. ${ }^{13}$ Hydropower, therefore, has an increasingly valuable role as part of a sustainable energy portfolio by not only producing renewable energy itself, but also by making it possible to incorporate larger amounts of other renewable generation resources, such as wind, with the balancing reserves it provides.

Unlike other power generating resources, however, hydropower facilities are generally designed and operated to serve multiple objectives. In addition to power generation, these objectives may include:

- Flood control

- Environmental objectives including wildlife habitat protection and fisheries management

- Water storage for agricultural, municipal and industrial uses

- Navigation

- Water quality

\footnotetext{
${ }^{12}$ High levels of wind penetration also have implications for the total system inertia which affects the requirements for primary frequency control reserves. Hydropower has the added benefit of contributing to higher system inertia, reducing the need for additional reserves. This benefit of hydropower is not explored in the work we present here. The reader is referred to Eto et al. (2010) for more information on the role of system inertia in maintaining sufficient frequency control reserves.

13 Only conventional hydropower is considered in the analysis we present, though the methodology could be extended to systems with PSH.
} 


\section{- Recreation}

The specific uses and objectives of hydropower facilities and their relative priorities are unique to each system, but one common characteristic is that these non-power, water objectives generally have a higher priority in system operating policy than the power objectives. That is to say that the water management objectives cannot be compromised for power generation purposes. The common effect of these non-power policies is that they restrict the allowable outflows from the hydropower facility, which has the effect of reducing system flexibility. So although from a technical perspective hydropower can provide a significant amount of flexible generating resources for balancing wind generation, the true system flexibility is effectively reduced by the non-power policy that governs operations of the system. While the hydropower system may still maintain a fair amount of operational flexibility within the constraints of the non-power policy, it is necessary to account for the reduced flexibility from the non-power constraints when evaluating the actual capacity of the hydropower system for balancing integrated wind generation.

Integrating hydropower and wind generation has valuable benefits for increasing the amount of renewable energy generation online. Operating hydropower systems to balance increased variability in net load, however, has impacts that must be considered as well. These include increased starts and stops for generating units as well as increases in ramping. This increases the wear on generating units which can shorten their expected life and increase maintenance requirements. In addition, generators may spend more time running off peak efficiency to ensure that sufficient incremental and decremental reserve capacity is online to balance variability in net lead. These operational impacts translate into economic costs for the hydropower system. ${ }^{14}$

In many regions, formal markets are now in place for both energy and ancillary services. ${ }^{15}$ Ancillary services include the reserves that are used to even out any imbalances between energy supply (generation) and demand (load). For hydropower systems that participate in these markets, the ancillary services they provide can often represent a significant percentage of total system

\footnotetext{
14 Mechanisms for compensating hydropower systems for these increased costs vary depending on power purchase agreements for the specific generating resources and market structures within the region.

15 The terms ancillary services and balancing reserves are used interchangeably throughout this text.
} 
revenue. If a higher level of reserves must be committed to support wind generation in the system, it may reduce the amount of capacity that is available to bid into the ancillary services market. In addition, the need to balance forecasted wind generation may shift the timing of hydropower generation in relation to energy prices and thus have an effect on the economic value of the energy generated by hydropower. These economic impacts are heavily dependent on the characteristics of each system including the market structures that are in place, the nature of the generating resources, and the organizational structure and contract agreements of the integrated hydropower and wind generating entities. These are effects that need to be considered when evaluating hydropower and wind integration.

The methodology we present here evaluates the integration of hydropower and wind generation in the context of realistic hydropower modeling including realistic non-power policy. The study uses the RiverWare hydropower modeling tool to optimize coordinated hydropower and wind generation within the policy constraints. Outputs evaluated include the economic value of energy and ancillary services from the integrated system as well as operational outputs such as power generation and reservoir outflows. In addition, the ability of the system to satisfy all policy constraints is evaluated. The methodology is applied to a test system for a range of wind penetrations with varied hydrologic conditions and varied levels of policy constraints. A sensitivity analysis is also performed on select system parameters to observe the response of the system to changes in key assumptions and system characteristics.

\subsection{Existing Work on Hydropower and Wind Integration}

With the rapid growth in wind generation and the push for more installed wind capacity to help meet RPS requirements, much work has been done to try to assess both the technical feasibility of integrating high levels of wind generation and the economic impacts of large scale wind integration. Parsons et al. (2006) provide a summary of wind integration studies carried out

in the United States by various utilities and independent system operators (ISOs). These studies evaluate the cost impacts of increased reserve requirements in the system due to the increased 
variability and uncertainty in net load inherent with added wind generation. Summarized results show integration costs ranging from $\$ 0.45 / \mathrm{MWh}$ to $\$ 4.97 / \mathrm{MWh}$. DeCesaro and Porter (2009) update the list of studies and include results that show integration costs as high as $\$ 8.84 / \mathrm{MWh} .{ }^{16}$ Results show that wind integration costs vary significantly with the level of wind penetration modeled and can be dependent on modeling assumptions within each system. It should be noted that while hydropower is included as one of the generating resources in many of these studies, and at times serves as the primary balancing resource, it is not the specific focus of these studies.

A number of studies have evaluated the benefits of utilizing the flexibility of hydropower systems to balance the variability and uncertainty of wind generation. Belanger and Gagnon (2002) provide one of the earlier studies considering the use of hydropower to balance wind generation in the Hydro Quebec system. Their analysis identifies the increased hydropower resources that would be required to balance variable wind generation as a "hidden cost" of wind. They also consider the impact of wind integration on stream flows in the hydropower system identifying reduced minimum flows and increased hourly fluctuations as potential impacts with higher levels of wind penetration. In another early study Jaramillo et al. (2004) calculate the firm power that could be provided by a single wind farm coupled with a single hydropower reservoir. Their analysis does not, however, consider the operation of the generating resources to meet a time-varying load. A case study in Finland presented by Holttinen and Koreneff (2012) shows that even run-of-river hydropower with limited storage has the ability to balance most wind forecast error and can reduce the imbalance cost from wind forecast error in the Nordic market.

In addition to studies that evaluate the benefits of hydropower for wind integration, another set of works focus on the development of algorithms to coordinate the scheduling of hydropower generators with wind and other generating resources. Angarita and Usaola (2007) develop an approach to optimize the combined operation of hydropower and wind resources that bid into an energy market. The objective is to maximize the combined profit of the hydropower and wind resources based on the price of electricity, the value of water and a penalty for energy imbalance.

\footnotetext{
16 The studies referenced in these reports can be found at http://www.uwig.org/opimpactsdocs.html.
} 
The hydropower resource is used to meet the imbalance from the wind forecast error only when the imbalance penalty would be greater than the value of the water used for generation to make up the imbalance. Results show the value of coordinated operations increases as the imbalance penalty increases. This strategy is extended in Angarita et al. (2009) to assess the value of combined bidding and operations of coordinated hydro and wind resources. In the latter work, wind is treated as a stochastic parameter, and the optimal energy bid is determined by calculating the optimal operational strategy for each wind outcome and weighting it by the probability of that outcome. The mathematical formulation of the optimal bidding strategy includes limits on the minimum and maximum outflow at each reservoir due to technical and external (policy) constraints. The algorithm is applied for a scheduling horizon of five hours.

Matevosyan and Söder (2007) present a two-stage planning algorithm to optimize coordinated hydropower and wind bidding into a spot market with transmission constraints while accounting for uncertainty in wind forecasts. The hydro resources are assumed to have transmission priority but can reduce their bid in the "re-planning" stage when congestion is expected. The wind farm pays the hydro producer for reducing production from its optimal uncoordinated hydro schedule in order to relieve congestion, and the hydro producer retains the water to generate at a later time. A case study demonstrates increased revenue for both the hydro producer and the wind farm and reduced wind curtailment when applying the coordinated planning algorithm. In Matevosyan et al. (2009) uncertainty in energy prices is included in the methodology as well as the option to bid into the regulating energy market.

Benitez et al. (2008) present a nonlinear constrained optimization model to schedule coal, gas and hydropower generators with added wind generation to minimize the cost of generation while meeting demand. The approach is applied to a case study of the Alberta Electric System Operator system, and the results show that increasing hydropower capacity can reduce the required output from an additional peak generator.

A methodology is developed by Karki et al. (2010) to coordinate hydropower and wind generation to meet energy demand. The method first determines if wind generation output is below 
a set coordinating threshold and commits available hydropower units if it is below the threshold. Additional available hydropower units are used as peaking units if the combined base generation, wind and hydro are still less than the load. Application of the methodology to a system with a single reservoir with six hydro units and a single wind farm shows that coordinating hydro units with wind can improve system adequacy when there is sufficient water. If the system is water limited, coordinating hydro units with wind reduces system adequacy as there is no longer sufficient peaking capacity available from hydropower units to prevent loss of load.

A methodology to optimize the scheduling of run-of-river hydropower plants using model predictive control to smooth the variability of wind generation is developed by Hug-Glanzmann (2011b). One significant feature of this methodology is that it directly models the routing of water through the cascading reservoir system using a linearized and discretized form of the Saint Venant equations. Constraints for the model include minimum and maximum values for river levels, hydropower releases and hydropower ramp rates. This model for the hydropower system is then combined with a natural gas plant, a coal plant, a nuclear plant, a wind plant and a storage device in a hybrid approach that optimizes weighted economic, environmental and quality of service objectives (Hug-Glanzmann, 2011a).

Additional studies have examined the potential benefits of coupling pumped-storage hydropower with variable wind generation (see for example Black and Strbac, 2006; Bueno and Carta, 2006; Kaldellis et al., 2010; Troy et al., 2010; Tuohy and O'Malley, 2009). The analysis we present, however, is restricted to conventional hydropower without pumping.

While utilizing hydropower to balance variable renewable generation can provide benefits to the overall power system, it also has impacts on the hydro system which must be considered. A case study of the Grant County Public Utility District hydropower resources on the Columbia River in Washington by Acker et al. (2012a) indicates that wind generation forecast error could lead to violations of system reserve capacity requirements and non-power flow constraints. The study suggests, however, that these potential violations could often be handled by system operators during operations or through real-time transactions. Piekutowski et al. (2012) discuss the impact 
of high levels of wind generation on hydro equipment based on a case study of the Tasmania power system. Balancing variable wind generation can lead to more frequent starts and stops of hydro units which increases wear and tear on electrical and mechanical components. Running hydro units off peak efficiency in order to maintain sufficient reserves can also cause problems from vibration and cavitation. Complete analysis of hydropower and wind integration should account for such factors.

Three large-scale studies on wind integration that have been completed in recent years also warrant mentioning here. In 2010 the National Renewable Energy Laboratory completed the Western Wind and Solar Integration Study (GE Energy, 2010) and its sister study the Eastern Wind Integration and Transmission Study (EnerNex, 2010). The WWSIS evaluates the feasibility and economic impacts of large scale renewable generation in the entire Western Interconnection. It specifically evaluates renewable integration scenarios with as much as $30 \%$ wind and $5 \%$ solar in the WestConnect footprint, which includes five western states, with $20 \%$ wind and $3 \%$ solar in the remaining six states of the Western Interconnection. With the large amount of hydropower resources in the western United States, modeling hydropower as a balancing resource for variable renewable generation is a significant part of the study. The analysis shows a significant increase in operating costs for the entire system if the flexibility of hydropower is removed. This is done by scheduling hydropower resources as a flat block of power instead of allowing it to be economically dispatched with the other generating resources. A significant cost increase is also shown if hydropower is scheduled based on load alone without renewables and thus its flexibility is not used to help balance the variability of the renewable generation. Hydropower modeling is not as significant a component in the EWITS, which evaluates scenarios with as much as $30 \%$ wind generation in the Eastern Interconnection. Results of the study did show, however, that increased hydropower storage could reduce the need for some of the additional transmission that would be required to accommodate high levels of wind generation.

The third major study is the IEA ${ }^{17}$ Wind Task 24 Integration of Wind and Hydropower

\footnotetext{
17 International Energy Agency
} 
Systems Final Technical Report (Acker, 2011a,b). This study brings together participants from seven different countries from North America, Europe and Australia to investigate wind integration in electric systems with large amounts of hydropower generation. The work is carried out as a set of case studies by each of the participating entities which analyze benefits, challenges and impacts of wind integration in systems with varied characteristics in their market structures, hydropower systems and electrical systems. Results from the case studies are summarized in Acker et al. (2012b) and indicate that in many cases existing hydropower systems can provide the balancing reserves necessary for integrating large amounts of wind generation. Case studies from the U.S. suggest that at wind penetrations around $20 \%$ operational difficulties related to meeting flow constraints and scheduling reserves could emerge for hydropower systems balancing wind.

\subsection{Significance of this Research}

Significant improvements have been made in wind and hydropower integration studies in recent years. There is still a need for improvement, however, particularly in the hydropower modeling. The IEA Wind Task 24 Final Report acknowledges the limitations of production cost models that are typically used in wind integration studies. The report calls for "improvements in how they model hydropower operation, water balances, and constraints." (Acker, 2011a, p. 100) The report also recognizes the effects that non-power constraints can have in limiting hydropower system flexibility in balancing integrated wind generation. Among its participants, however, it found non-power constraints to have a significant impact on reducing system flexibility only within the United States.

In the WWSIS hydropower operations and the water in the hydro facilities are not modeled directly. Generation schedules are constrained to meet historic average monthly generation totals while remaining within historic monthly minimum and maximum levels (GE Energy, 2010). While

this approach may be useful for a large scale study like the WWSIS, it does not capture the impacts on operations at the individual system or plant level, nor does it account for restrictions on capacity from varying storage levels or operational constraints. In a companion study by Acker 
and Pete (2012) that carries out a detailed analysis of the hydropower component of the WWSIS, it is recognized that modeling assumptions at times lead to an overestimation of true hydropower capacity, particularly in low flow years, and thus at times hydro generation is modeled at levels that are higher than what was realistically feasible. The report concludes with this statement:

[T] his study also revealed that while the hydro system can be reasonably modeled, there are several modeling limitations related to capturing non-power regulations and constraints that often govern hydro flexibility and availability. The need for production cost models to capture or incorporate these factors is apparent particularly at the individual hydro plant level and especially as wind and solar integration studies like the WWSIS become increasingly comprehensive and consider large penetrations of renewables. (p. 9-5)

In an additional summary of the impact of high wind penetrations on hydropower in the WWSIS, Hodge et al. (2011) state, "It is important to note that the operation of hydro units is often strongly influenced by non-power considerations. To more accurately establish the effects of high wind penetrations on hydro system operations, these non-power constraints must be modeled on a unit-by-unit basis." (p. 8)

The research we present here contributes to this end by developing a methodology, described in detail in Chapter 2, that assesses the value of wind generation integrated with hydropower that not only models the physical processes of the hydropower system but also accounts for the limitations resulting from both power-related and non-power policy constraints. Previous hydropower and wind coordination algorithms typically include hydropower constraints on minimum and maximum storage volumes and minimum and maximum plant discharges (see Angarita et al., 2009; Benitez et al., 2008; Matevosyan et al., 2009; Karki et al., 2010; Hug-Glanzmann, 2011b). In general, however, these constraint values are static and represent the physical characteristics of the system. One exception is in Angarita et al. (2009), which includes time dependent minimum and maximum release amounts corresponding to external constraints. Also Hug-Glanzmann (2011a,b) constrains river elevations to minimum and maximum values and includes the minimization of deviations from a reference value as an environmental objective in the weighted objective function. In the strictly run-of-river model (no storage) developed there these are the equivalent of 
environmental flow constraints and objectives.

The methodology we present here utilizes the pre-emptive linear goal programming optimization solver in RiverWare to model hydropower operations with a set of prioritized policy constraints and objectives based on realistic policies that govern the operation of actual hydropower systems, including licensing constraints, environmental constraints, water management and power objectives. This approach accounts for the fact that not all policy constraints are of equal importance. For example target environmental flow constraints may not be satisfied if it would require violating license minimum or maximum storages (pool elevations), but environmental flow constraints will be satisfied before optimizing power generation. The methodology allows for the evaluation of the effective capacity and flexibility of a hydropower system that must be considered by system operators when coordinating hydropower resources with variable and uncertain wind generation.

In addition to realistic modeling of hydropower operations and policy, the methodology also produces an economic evaluation of integrated wind and hydropower that not only accounts for the value of energy from the integrated system but also for the value of ancillary services provided by the hydropower resources. As mentioned previously the increased variability and uncertainty in net load inherent with increased wind penetration levels requires an increase in operating reserves or ancillary services (see, for example Ela et al., 2011; Acker, 2011a). With the relatively recent development of markets for ancillary services in many regions, ancillary services can carry an explicit economic value in addition to their value in maintaining grid stability. A significant portion of hydropower revenue can result from providing ancillary services, and thus ancillary services should be accounted for when determining the total value of the hydropower system. Our methodology provides a means for assessing the impact of wind generation on the value of ancillary services within the system.

The end value of integrated hydropower and wind generation is specific to each power system and is dependent on numerous factors including, but not limited to, the wind penetration level, the physical characteristics of the hydropower system, geographic distribution of wind resources, weather patterns, hydrologic conditions, the mix of thermal generation resources, available trans- 
mission, demand patterns, market structures and hydropower operational policy. Any of these factors can vary widely from one system to another. In consideration of the specificity of the value of wind generation to each system, the goal of our study is not to quantify a specific value for integrated wind generation. The point is rather to demonstrate the importance of modeling realistic hydropower operations, including non-power constraints, when considering the value of integrated hydropower and wind generation. In addition the sensitivity of the value of hydropower and wind integration to various conditions and system parameters is evaluated. To do this, our proposed methodology is applied to a sample hydropower system with integrated wind generation, described in Section 2.4, under a range of scenario conditions and values for select model parameters. By comparing effects on economic outputs, operational conditions and constraint satisfaction from modifying various factors and scenario conditions, conclusions are drawn about key modeling assumptions and system characteristics and their effect on the evaluation of hydropower and wind integration.

The installed capacity of renewable generating resources continues to grow. This is motivated by a combination of factors including the need to meet higher power demands, economic incentives and state RPS requirements to provide clean energy. These renewable resources provide numerous economic, social and environmental benefits. At the same time, adding more variable and uncertain generation such as wind pushes power systems closer to their limits of operational flexibility. It is important to understand both the economic and technical limits to the benefits of added wind generation. With reduced margins in operations, it becomes increasingly important to accurately model the full operational flexibility and limitations of balancing resources in the system. When hydropower is being modeled as a balancing resource, it is also critical to account for the fact that these resources are not operated in isolation but are part of a larger, integrated environmental and water management system. The methodology we present provides a framework to evaluate the benefits and limitations, both economic and operational, of integrating hydropower and wind generation. It allows for a more reasonable assessment of the effects of wind generation on hydropower operations and makes it possible to improve on many of the approximations and assumptions made 
about hydropower resources in previous wind integration studies. These improvements can then be used to provide a better representation of power system characteristics for future wind integration studies. 


\section{Chapter 2}

\section{Modeling and Methodology}

This chapter describes the proposed methodology for assessing the value of integration hydropower and wind generation. First the conceptual model is presented followed by a description of the run sequence used to simulate the scheduling and operation of actual hydropower systems. This is followed by the details of how the conceptual model is implemented in RiverWare. Then a description is given of the test system, input data and scenarios modeled to evaluate the effects of wind integration on the hydropower system under a range of conditions.

\subsection{Conceptual Model}

\subsubsection{Model Overview}

The manner in which a hydropower system is scheduled and operated is dependent to a significant degree on the structure of the larger system of which it is a part. The organizational structure of the larger system is highly dependent on the type of market structure which exists in the BA in which the system is located. In this study we do not attempt to model all possible market or organizational structures, so it is necessary to define the organizational model that is assumed for the purposes of this work. It is also necessary to identify the reasons for modeling systems assuming a particular organizational structure and the limitations of that organizational model. The most complete representation of integrated hydropower and wind generation would come from modeling a complete BA including all generation resources, load centers and transmission in addition to modeling the complete hydropower system. Such a model is beyond the scope of this study. Still, 
in order to maintain realism, the methodology should capture the interactions of multiple generation sources and system load, assuming that net load is balanced across a range of resources as opposed to simply modeling a single hydropower plant balancing a single wind farm (Milligan et al., 2011). The methodology must also be able to capture the effects of varying levels of wind generation on the system.

The organizational model we employ here is a hybrid of a traditional, vertically integrated utility $^{1}$ and a formal market. The system modeled is a sub-system of a BA such as a large-scale utility which owns multiple hydropower resources. These resources must be dispatched to meet local load and reserve requirements. Additional capacity can be utilized to bid into formal markets for energy and ancillary services. Such a mixed model is representative of organizational structures in parts of the Western Electric Coordinating Council (WECC) where power producers must meet load obligations in their own BA but also have the opportunity to participate in the California Independent System Operator (CAISO) market (Loose, 2011). Independent system operator (ISO) and regional transmission operator (RTO) markets have been identified as mechanisms to improve conditions for integrating large amounts of wind generation by providing sub-hourly energy markets that redispatch every five to fifteen minutes (Milligan et al., 2009). A schematic diagram of the organizational model we employ is shown in Figure 2.1.

In our model, participation in the market occurs in only one direction. Energy and ancillary services can be sold into the market when the hydro system has excess capacity, but they cannot be purchased from the market to help meet local requirements. Generally, the hydropower system we model has sufficient capacity to meet the local load requirements, so there is no "need" for the utility to purchase energy from the market when scheduling generation. ${ }^{2}$ The potential "need"

\footnotetext{
${ }^{1}$ A vertically integrated utility is a utility that owns both generating and transmission resources and has a load within its boundaries that it is obligated to meet with those resources. It will typically try to manage its resources to minimize cost while meeting its load. This is distinct from BAs with formal markets where individual power producers do not have their own load that they must meet. In BAs with markets, power producers bid energy into the market and the ISO schedules the generation from multiple power producers to meet the load.

2 While the utility we model has sufficient resources to meet its own load, there could be circumstances in which the economically optimal schedule would include purchasing energy and reserving capacity in the hydropower system. The CAISO market does support static hourly scheduled interchanges with external BAs (see http://www.caiso.com/2476/2476ecfa5f550.pdf); however, the use of such resources in scheduling the system is not explored in our research. The effects of co-optimizing local resources with energy purchases could be evaluated in
} 
for external resources purchased from the market would occur during real-time operations when actual wind and load deviate from the forecast. Currently the CAISO market only allows dynamic scheduling $^{3}$ of imports into its BA but not dynamic scheduling of exports. In order to represent the unavailability of these real-time resources (dynamically scheduled exports) from the market, in our study all resources to meet local requirements must come from within the utility being modeled.

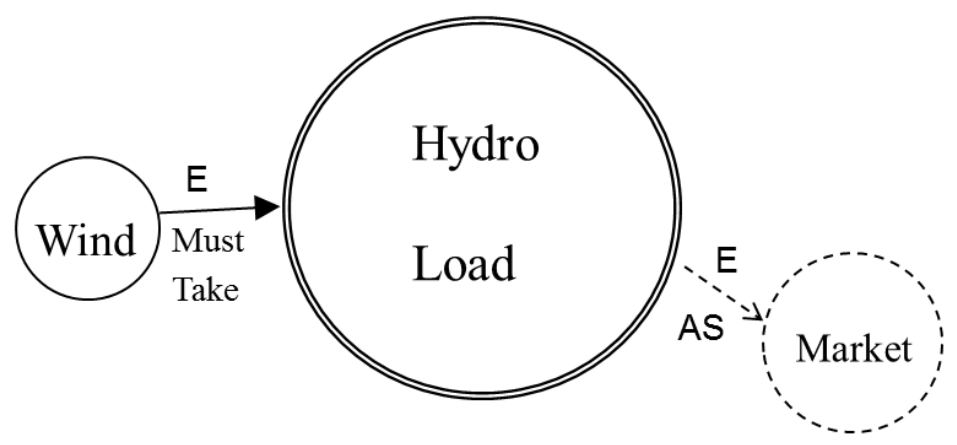

Figure 2.1: Conceptual Organizational Model: Hybrid of a traditional vertically integrated utility (the double-lined circle) with must take wind contracts and formal markets

The utility is assumed to have must-take contracts with a set of geographically dispersed wind farms. Wind is incorporated into the model as negative load. One important note is that no explicit economic value is assigned to the energy and reserves used to meet the local load and reserve requirements. Meeting the local load and reserve requirements is treated as a constraint for the system. Only the energy and ancillary services sold into the market are given an explicit economic value. ${ }^{4}$ Wind generation that is integrated as negative load reduces the amount of hydro capacity that must be utilized to serve load and potentially allows more energy to be sold into the market, which is the source of the value added from wind integration.

future research.

3 Dynamic scheduling refers to the intra-hour scheduling of resources (generation imports and exports) between BAs, typically at 4-second intervals. The CAISO market allows for dynamic scheduling of imports into its BA. The participation of our utility in the ancillary services market fits into this category. CAISO does not allow dynamic scheduling of exports. These are the resources our utility would potentially utilize from the market if available to balance real-time deficiencies in capacity due to wind and load forecast error. See http://www.caiso.com/2476/2476ecfa5f550.pdf.

4 Adding an economic value to the energy generated to meet local load could easily be done within the existing methodology and is only a matter have having appropriate price data; however, this would not affect the optimization solution or the relative results between scenarios. 
Many previous wind integration studies have evaluated the integration cost for wind (see Parsons et al., 2006). The baseline scenarios for such studies typically include the equivalent energy that would be provided by the added wind generation but as either a constant flat block or as flat blocks that represent the average wind generation over a constant interval such as 6 or 24 hours. Milligan and Kirby (2009) address the drawbacks of such an approach in establishing a baseline. Modeling a constant flat block of energy tends to result in more generation during peak prices resulting in an unrealistically high value for the base case scenario, and thus the cost of wind integration is overestimated. The shorter blocks reduce this problem but introduce unrealistic step changes in generation when transitioning from one block to the next.

The IEA Wind Task 24 Final Report states that wind integration studies should not only consider the integration cost due to the increased balancing requirements that come with increased variability and uncertainty. Rather a cost-benefit analysis should be carried out which considers the overall impact of wind integration on the power system (Acker, 2011a). In this regard, in the approach we present here, the net value of wind is assessed as opposed to the integration cost alone. The baseline for each scenario is simply the No Wind case. Wind is not assigned a purchase cost in our model. ${ }^{5}$ Such a cost is specific to each system and depends on who owns the wind generating resources and the power purchase agreements that are in place. Rather the output returns the net value of added wind generation (without subtracting a purchase cost) by comparing the total value from a Base case without added wind generation to the same scenario with wind generation. This theoretically represents the maximum price the utility would be willing to pay to integrate the additional wind generation. No attempt is made in our analysis to determine how the net value from integrating additional wind generation should be allocated among the various entities. ${ }^{6}$ This is again dependent on the contract arrangements within a given system. The results do provide a breakdown of the various components of value in the system: the market value of energy, the

\footnotetext{
${ }^{5}$ A purchase cost for wind could easily be incorporated into the methodology if the appropriate price data are available.

${ }^{6}$ Zima-Bockarjova et al. (2010) develop an algorithm to calculate how profits should be shared from a coordinated hydropower and wind bidding strategy. Milligan et al. (2011) address this issue in the context of cost allocation.
} 
market value of ancillary services and the value of energy in storage. This allows for an assessment of how wind integration impacts each of these value components. Section 2.4.4.3 describes how the individual impacts of energy, variability and forecast error are isolated from the total net value of added wind generation.

\subsubsection{Ancillary Services}

The classification and nomenclature of ancillary services or operating reserves vary in different regions and parts of the world (Ela et al., 2011). It is therefore necessary to clarify how ancillary services terminology is used in our model. Five separate ancillary services are defined in our model: Regulation up, regulation down, load following reserve, spinning reserve and non-spinning reserve. Details about how these ancillary services are incorporated into the RiverWare model are given in Section 2.3.3. Regulation, including both regulation up and down, represents the reserves used to balance rapid fluctuations (sub-seconds to minutes) in net load. In actual operations these reserves are deployed by automatic generation control (AGC). In our model regulation up, the capacity to increase generation to balance an increase in load, is quantified separately from regulation down, the capacity to decrease generation in order to balance a decrease in load. This is consistent with the classification in the CAISO market. ${ }^{7}$ Other regions, such as the $\mathrm{PJM}^{8}$ market and the MISO ${ }^{9}$ market, combine regulation up and regulation down into a single, bi-directional reserve. Our model accounts for both the regulation capacity, the amount of capacity that is reserved for regulation, and the regulation deployment energy, the amount of actual generation (or actual reduction of generation in the case of regulation down) when regulation capacity is actually deployed to balance total generation with load. The need for regulation is assumed to come from fluctuations in the load. Wind does not typically contribute to an increase in net load variability in the second-tosecond time scale at which regulation is deployed. Wind tends to contribute to variability on the time scale of minutes to hours (Ela et al., 2010). The requirement for regulation in our model,

\footnotetext{
${ }^{7}$ http://www.caiso.com/market/Pages/ProductsServices/Default.aspx

${ }^{8}$ http://www.pjm.com/markets-and-operations/ancillary-services.aspx

${ }^{9}$ https://www.midwestiso.org/MarketsOperations/RealTimeMarketData/Pages/AncillaryMarketMCP.aspx
} 
therefore, does not change as the amount of wind penetration changes.

Spinning reserve and non-spinning reserve together make up the contingency reserve. This represents the reserve capacity that can be deployed in the case of a major contingency event such as the unplanned outage of a major generating source or loss of a major transmission line. Spinning reserve represents the reserve that is on-line, synchronized and can ramp up rapidly to provide the full reserve capacity, whereas non-spinning reserve represents the reserve that can be brought on-line, synchronized and run at full capacity within a short amount of time, typically ten minutes. A certain percentage, typically $50 \%$, of contingency reserve is required to be composed of spinning reserve (WECC, 2008). One note is that regulation and spinning reserve are classified separately in our model. That is to say that any reserve capacity that is designated as regulation up cannot also be designated as a portion of the spinning reserve. The amount of contingency reserve within a BA must be at least as much as the largest single possible contingency (WECC, 2008). The contingency reserve requirment also is not affected by the level of wind penetration (Holttinen et al., 2008); however, the amount of contingency reserve that must be provided by the hydropower system is a key assumption that has a significant impact on the results of wind integration modeling. Scenarios are modeled with the contingency reserve requirement set at different levels as part of the sensitivity analysis.

Load following reserve is similar to regulation in that it is used to balance deviations in the net load from the scheduled generation but at a longer time-scale, tens of minutes to a few hours. It is typically at this time scale that wind variability and forecast error have the greatest impact on the system; therefore load following is the main ancillary service that is directly impacted by increased wind penetration. ${ }^{10}$ Load following reserve, as it is used in our model, represents what may be a combination of several classes of reserves in other systems including following reserves, balancing reserves and ramping reserves depending on the nomenclature within each system. Section 2.4.3.5

\footnotetext{
${ }^{10}$ In some systems, the amount of regulating reserve is increased in addition to the load following reserve based on the level of wind penetration, variability and uncertainty (see, for example, Holttinen et al., 2008). In the work we present here it is assumed that all increased reserve capacity for balancing wind generation is incorporated into the load following reserve.
} 
details how the load following requirement is set in our model.

\subsubsection{Markets}

The energy and ancillary services markets in our model are based on components of the CAISO market. In day-ahead energy markets, power producers submit bids which consist of a quantity of energy they are willing to commit to produce and the corresponding price they are willing to take for that energy. For day-ahead markets, bids are typically at hourly intervals. For the CAISO day-ahead energy market, bidding for a given day opens one week prior to the day of generation and closes the day prior to the day of actual generation. ${ }^{11}$ Once the market closes, the ISO (or equivalent market operator in other markets) ranks the bids for each hour by price. Bids are awarded beginning with the lowest price and proceeding through higher prices until the amount of energy in bids awarded matches the forecasted load for the given hour. When the awarded bids match the demand, the market is said to have "cleared." Once the market has cleared, the ISO releases the generation schedule for each hour, which lists the generating units and the amount of energy they are committed to provide (corresponding to the bid awarded). ${ }^{12}$ Once the generation schedule for the hour is published, each power producer with an awarded bid (committed unit) is required to produce that energy at the scheduled hour or face penalties if they are unable to provide the committed generation. All generation from the day-ahead market for a given hour is compensated at the highest awarded bid price for the hour, the market clearing price (MCP). The term 'day-ahead energy' as used throughout this text refers to energy committed by the hydropower system to the day-ahead market.

The CAISO day-ahead market sets hourly market-clearing prices for energy and ancillary services. ${ }^{13}$ It also schedules unit commitments for the following day. The model we present here includes hourly prices for energy and each ancillary service. The optimization solution schedules

\footnotetext{
${ }^{11}$ http://www.caiso.com/market/Pages/MarketProcesses.aspx

12 CAISO day-ahead market results are published at 1:00 p.m. of the day prior to generation.

13 The CAISO market also includes products for residual unit commitment, congestion revenue rights and convergence bidding which are not included in our model. http://www.caiso.com/market/Pages/ProductsServices/Default.aspx
} 
generation and reserves across the hydro system to maximize the combined revenue from energy and ancillary services based on the hourly prices and the available capacity after meeting all local requirements. In select scenarios a modified real-time market is also included in the model. The CAISO real-time market operates in five minute intervals. The hourly time step model we present approximates this by averaging real-time prices and energy over each hour.

The marketable ancillary services in our model include regulation up, regulation down, spinning reserve and non-spinning reserve, consistent with the ancillary services products in the CAISO market. In our model, ancillary services can only be sold into the day-ahead market, though CAISO also has a real-time market for ancillary services. Load following reserve is not modeled as a marketable ancillary service but is used only as a local resource to balance deviations in the net load from the scheduled generation. Ancillary services are compensated for both the capacity sold into the market and for the energy generation in the case that the ancillary services are deployed. The capacity is compensated at the marginal price for the given ancillary services. In our model the deployment energy is compensated at the real-time marginal energy price. For regulation down, the deployment is a reduction in generation from the schedule and thus a loss of immediate revenue from energy using the day-ahead marginal energy price as the unit cost of the reduction in generation; however, the water that would have been utilized for that generation remains in the reservoir and can be used to produce revenue by generating at a later time.

In reality, only small amounts of energy can be bid into a market before displacing more costly generating sources and thus reducing the market clearing price. Likewise, high levels of wind generation can result in a decrease in energy prices. Jónsson et al. (2010) show significant reductions in the day-ahead electricity prices in northern Europe's Nord Pool market when forecasts predict high levels of wind generation. Klinge Jacobsen and Zvingilaite (2010) also demonstrate increased price volatility in areas with high wind penetration in Denmark. Ignoring the impacts on energy prices due to large levels of energy being sold into the market would overestimate the value of added wind generation. In the methodology we present here, the impact of wind generation on energy prices is not modeled directly, that is to say that there is no a priori correlation between 
wind generation and energy prices. Rather, to model the effect on prices, it is assumed that the hourly marginal price of energy decreases by a set percentage for each additional $50 \mathrm{MWh}$ of energy that are sold at each time step. In this manner the model evaluates the impact on market prices of additional energy from any source, not exclusively wind generation. This also prevents the optimization solution from flooding the market with energy at any given time step. (Details on how the energy market is modeled are given in Section 2.3.4.) Alternative scenarios evaluate the impact of various levels of market depth by adjusting the parameter which sets the amount by which prices decrease for each additional amount of energy sold into the market. A similar reduction in market prices for ancillary services is modeled to prevent unrealistic flooding of the ancillary services market. Perfect forecasts are assumed for all price inputs in our model. (Matevosyan et al. (2009) present an algorithm for a coordinated hydropower and wind bidding strategy which incorporates the stochastic nature of energy markets.)

\section{$2.2 \quad$ Run Sequence}

The modeling methodology we present is intended to simulate the nature of actual hydropower operations. Hydropower resources are scheduled based on net load forecasts, hydrology, and system constraints. Then during real-time operations, generation must be adjusted from the schedule to meet actual conditions as they deviate from the forecast. In our model this is represented by a twostage optimization, a one week scheduling run using load and wind generation forecasts, followed by a 24 hour operations run using actual load and wind inputs. This sequence is illustrated in Figure 2.2.

The purpose of a one-week scheduling run is to ensure that the system is looking far enough into the future that it does not optimize near-term operations at the expense of compromising its ability to meet system requirements in the coming week. Operations for the current day must be planned not only to optimize for current conditions but also in anticipation of conditions in future days. A target ending storage constraint is included in the scheduling run for a similar reason. It prevents the optimization solution from draining all water from the hydropower system to maximize 


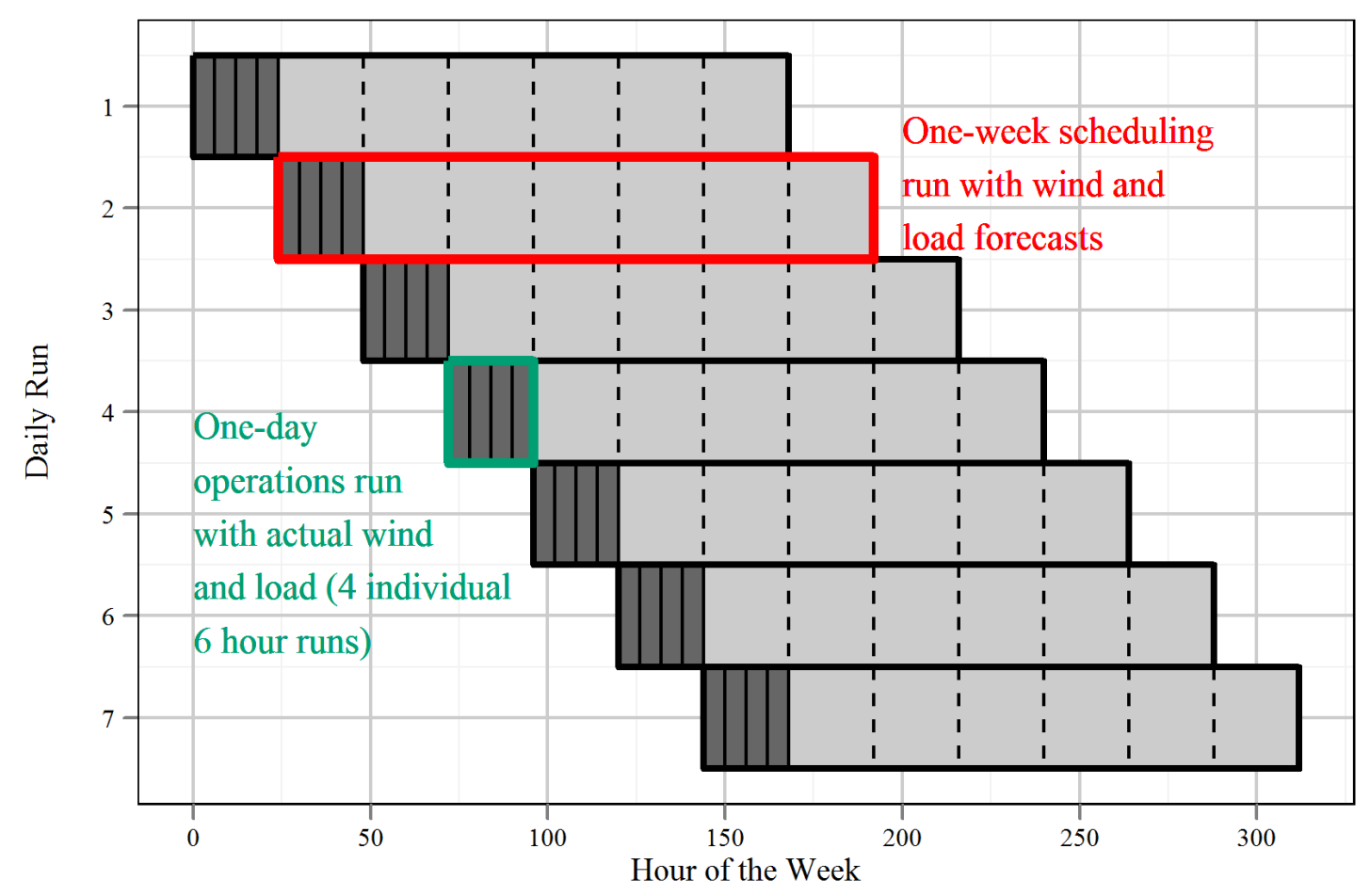

Figure 2.2: Diagram of the run optimization sequence: A one-week scheduling run followed by a 24-hour operations run divided into 4 individual 6-hour runs

revenue in the current week. The system must balance the objective to maximize revenue with the need to maintain sufficient operational flexibility at the end of the run to meet any conditions that may arise in the following week. The ending storage constraints are used to accomplish this. The optimization objective of the scheduling run is to maximize the total value of energy and ancillary services after meeting all non-power constraints, target ending storage constraints and all local load and reserve requirements. The energy sold into the market from the first 24 hours of the scheduling run represents energy committed in the day-ahead market (referred to further as 'day-ahead energy').

Total system outputs from the scheduling run include day-ahead energy and ancillary services sold into the market at each hour for the first 24 hours of the run. In addition to these system-wide outputs, individual hydro plant outputs include the releases, generation and reserves (ancillary 
services) for each of those 24 hours. These outputs represent the schedules for these plants for the next 24 hours. Storage volumes for each reservoir at the end of each six hour block are also taken as target scheduled storage values.

These outputs from the scheduling run become inputs for the 24 hour operations run. ${ }^{14}$ Other inputs to the operations run include actual load and actual wind generation, replacing the forecasts used in the scheduling run. Hydrologic inputs remain the same for the scheduling run and the operations run. A perfect hydrologic forecast is assumed over the one-week period. The hydrologic forecast is another potential source of significant uncertainty for some systems, the effects of which are not explored in the work we present here. The objective of the operations run is to maximize the value of stored water at the end of the run period after meeting all non-power constraints, load and reserve requirements, and target ending storage values.

The energy and ancillary services committed in the day-ahead market from the scheduling outputs are included as part of the load and reserve requirements that must be met by the hydro system in the operations run. The load following reserve requirement is removed representing the use of reserves to balance any error from the load or wind generation forecast. A soft constraint forces the system to attempt to meet the power and ancillary services demand with the scheduled power and reserves at each hydro project. This is in place to prevent the system from completely re-optimizing with the new inputs and simulates the loss of efficiency that can occur when the system must adjust operations to meet real-time conditions that deviate from the schedule. For select scenarios, a real-time energy market is included in the operations run, and the objective to maximize the value of stored water is replaced with an objective to maximize the value of real-time energy while meeting target ending elevation constraints.

Hydropower resources possess a significant amount of flexibility to adjust short-term schedules and real-time operations to meet changing conditions based on updated forecasts and operator judgment; however, modeling the operations run with a single optimization solution would overestimate this flexibility by simulating perfect forecast knowledge over the entire 24 hour time horizon.

\footnotetext{
${ }^{14}$ Note that the scheduling run and corresponding operations run begin on the same time step (hour).
} 
In order to prevent modeling perfect forecast knowledge yet still capture reasonable system flexibility, the 24 hour operations run is divided into four individual six hour optimization runs. With this approach, a perfect forecast is still modeled within each six-hour block. The re-positioning of the system within this six hour block, within the constraints imposed on the system, approximates the flexibility of the hydropower resources. The system has no knowledge, however, of conditions for the following six hour block, and therefore must re-adjust itself to meet the new conditions at the start of each new six hour run.

Outputs from the operations run include the economic values of day-ahead energy and ancillary services sold at each time step as well as operational data such as generation, releases, storage, and pool elevations for each reservoir at each time step. At the end of the 24-hour operations run, the model advances to the start of the next day. Updated load and wind forecasts are imported into the model, and the process repeats with a new one-week scheduling run. The reservoir storages from the end of the operations run become the initial conditions for the succeeding scheduling run. A diagram showing the flow of the two stage optimization sequence is shown in Figure 2.3. 


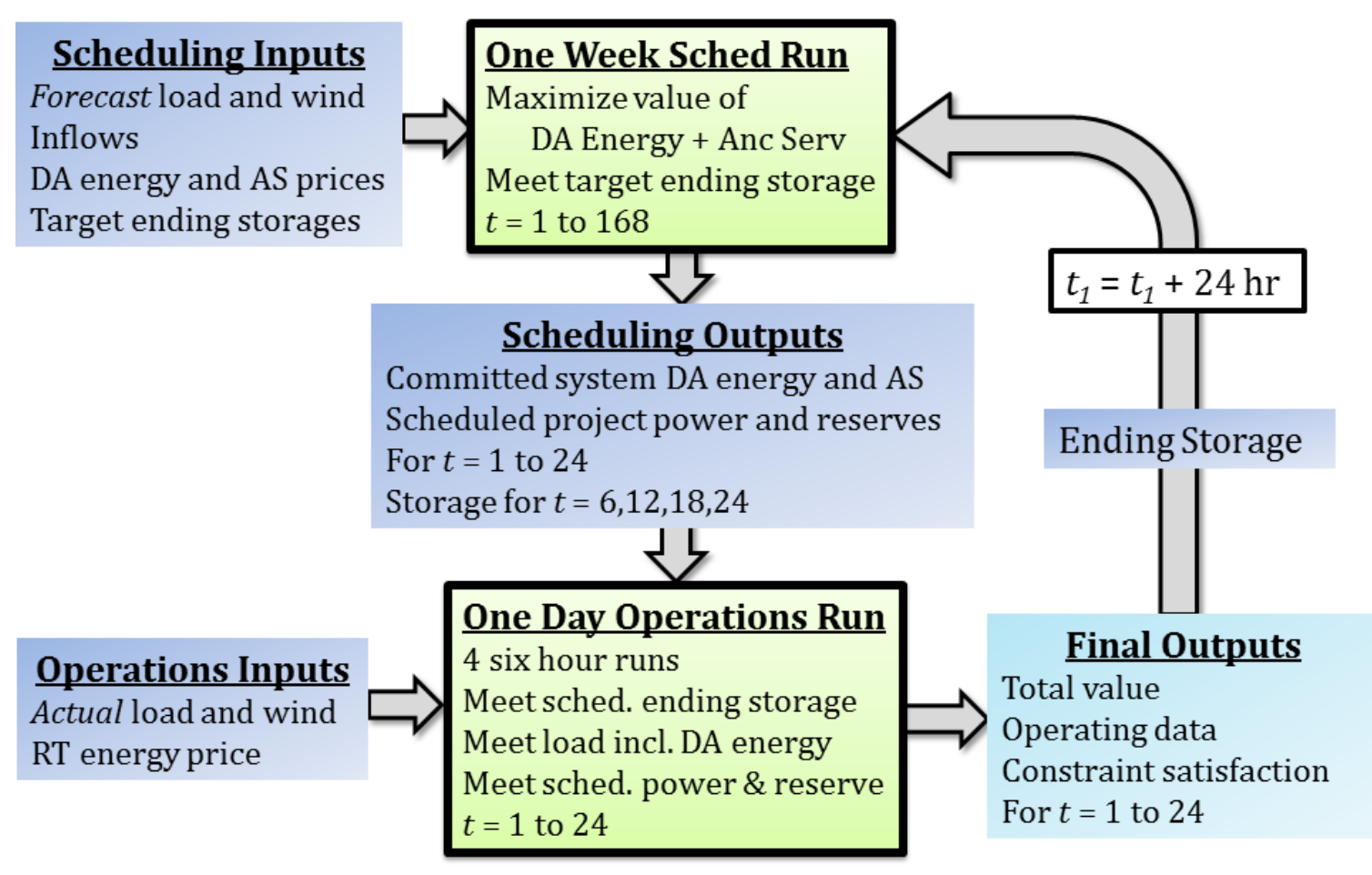

Figure 2.3: Diagram of the two-stage optimization sequence: A one-week scheduling run followed by a 24 -hour operations run divided into 4 individual 6 -hour runs 


\subsection{Hydropower Modeling in RiverWare}

Hydropower modeling for our study is carried out using RiverWare, a general tool for river and reservoir system modeling developed at the Center for Advanced Decision Support for Water and Environmental Systems (CADSWES) at the University of Colorado. A specific model in RiverWare is created by combining objects such as power reservoirs and river reaches on a workspace. The user then selects appropriate methods, physical process algorithms, for each object and provides parameter values for the object and selected methods. RiverWare has been implemented for planning and operations by agencies such as the Tennessee Valley Authority, the U.S. Bureau of Reclamation, and the U.S. Army Corps of Engineers. For a more complete general description of RiverWare and examples of its uses the reader is referred to Zagona et al. (2001). A detailed description of the physical process algorithms and modeling capabilities of RiverWare can be found in the online RiverWare Documentation.$^{15}$

\subsubsection{RiverWare Optimization - Preemptive Linear Goal Programming}

Our study utilizes RiverWare's optimization solution - a pre-emptive linear goal program. The user creates prioritized goals, which are constraints and objectives that represent the operating policy of the system. Then a linear program is solved at each priority level. When the goal is written as a soft constraint (or multiple soft constraints), the objective function of the linear program at that priority is to minimize the deviations from the constraint values. In the case that not all soft constraints at a given priority level can be satisfied, the optimization minimizes the maximum deviation, fixes the satisfaction variable for that constraint, then re-solves the linear program until all constraints at that priority are satisfied or all deviations are minimized. (This is known as a repeated minimax solution. Other solution approaches exist in RiverWare when multiple soft constraints are unsatisfied at a given priority level, but only the repeated minimax approach is used in our study.) Once a soft constraint has been satisfied at a higher priority, it will not be violated

\footnotetext{
${ }^{15}$ http://cadswes.colorado.edu/PDF/RiverWare/documentation/
} 
to meet a lower priority soft constraint or objective. Additional information about optimization in RiverWare, including the automatic formulation of physical constraints, is provided by Eschenbach et al. (2001).

Each optimization solution in RiverWare is followed by a post-optimization rule-based simulation. Rule-based simulation in RiverWare uses rules, logical "if-then" statements, to set selected input values. With the combination of input data and the values set by rules, each object is fully determined, and the model can solve for all output values. For example, if storage in a reservoir is known for the previous time step and all inflows are given as an input for the current time step, then if the releases at the current time step are set by a rule, the storage at the reservoir object can be solved for at the current time step.

One important distinction between the optimization solution and the post-optimization simulation is that the optimization returns a single global solution for all variables at all time steps in the run whereas the post-optimization simulation solves the system on a sequential, time step by time step basis. The optimization solution does not return the solution values to workspace. Rather the post-optimization rule-based simulation sets select values from the optimization solution and solves for the remaining values in the system. In doing so, minor adjustments are made to correct for approximation errors in the optimization solution. For example the power calculation in the optimization solution is based on a fixed operating head, but the post-optimization solution is able to use an iterative process to solve for power based on the operating head at each time step. The post-optimization rules used in our model set the optimization solution values for turbine release, spill and ancillary services. All other values, such as power generation are solved for in the post-optimization rule-based simulation based on the user-selected methods. ${ }^{16}$

\footnotetext{
${ }^{16}$ In certain cases, the rules set power values instead of turbine release in order to allow ancillary services values to be set correctly. The associated turbine release is then solved for through an iterative process.
} 


\subsubsection{Power Modeling}

Our study uses the plantEfficiencyCurve method in RiverWare to model power generation at each hydropower project. Power, $P_{r, t}$, at a given reservoir $r$ and time $t$ is modeled as a function of operating head, $O H_{r, t}$, and turbine release, $Q_{r, t}^{T}$.

$$
P_{r, t}=f\left(O H_{r, t}, Q_{r, t}^{T}\right)
$$

Plant data are entered in a plant power table in blocks of constant operating head with varying turbine release and corresponding power values. The result is a set of piecewise linear curves, one for each operating head. Linear interpolation between the data points is used to calculate power. In order to maintain linearity in the formulation of the optimization problem, a single value is assumed for operating head, $O H_{r}^{\text {opt }}$, at each reservoir, and thus a single piecewise linear curve is

used to calculate power, $P_{r, t}^{o p t}$, at each plant in the optimization solution. The single operating head value is set by the user based on the expected operating head at that reservoir during the run.

$$
P_{r, t}^{o p t}=f\left(O H_{r}^{o p t}, Q_{r, t}^{T}\right)
$$

In the post-optimization rule-based simulation, an iterative solution calculates the operating head as the difference in the forebay elevation and the tailwater elevation (a function of the outflow). This operating head value is then used to calculate power generation based on the linear interpolation represented by equation 2.1. This removes the approximation error introduced by assuming a constant operating head in the optimization solution.

\subsubsection{Ancillary Services}

For the mathematical formulation in RiverWare, ancillary services are divided into two classes: incremental reserves, which include regulation up, spinning reserve, non-spinning reserve and load following reserve, and decremental reserves, which include regulation down and load following reserves. Note that load following reserve, as we use it here, is considered to be a bidirectional reserve and carries the same amount of incremental and decremental reserve capacity. In general 
the incremental and decremental reserve capacities at a given hydropower plant $r$ and time step $t$ are constrained by

$$
\begin{gathered}
R U_{r, t}+S R_{r, t}+N R_{r, t}+L F_{r, t} \leq H C_{r, t}-P_{r, t} \\
R D_{r, t}+L F_{r, t} \leq P_{r, t}
\end{gathered}
$$

In equations 2.3 and 2.4, $R U_{r, t}, R D_{r, t}, S R_{r, t}, N R_{r, t}$ and $L F_{r, t}$ represent regulation up capacity, regulation down capacity, spinning reserve, non-spinning reserve and load following reserve respectively at reservoir $r$ and time $t . P_{r, t}$ is the power generated at reservoir $r$ at time $t . H C_{r, t}$ is the hydro capacity, the maximum potential generation at time $t$, and is defined in equation 2.6. In the optimization goal set, additional constraints can be added that further limit the incremental reserve capacity based on flow restrictions which effectively reduce the hydro capacity (see Section 2.4.2.3). Likewise, projects may have minimum generation requirements, in which case the constraint for decremental reserves becomes

$$
R D_{r, t}+L F_{r, t} \leq P_{r, t}-P_{r, t}^{\min }
$$

where $P_{r, t}^{\min }$ is the minimum generation requirement.

The hydro capacity at reservoir $r$ and time $t$ is based on a linear interpolation from the plant power table (see Section 2.3.2) and is function of the maximum turbine release, $Q_{r, t}^{T \max }$, and the operating head corresponding to the maximum turbine release, $O H_{r, t}^{H C}$.

$$
H C_{r, t}=f\left(O H_{r, t}^{H C}, Q_{r, t}^{T \max }\right)
$$

The maximum turbine release, however, is a function of the operating head, so this must be calculated through an iterative procedure:

$$
\begin{gathered}
Q_{r, t}^{T \max }=f\left(O H_{r, t}^{H C}\right) \\
T W_{r, t}^{H C}=f\left(T W_{r, t}^{B}, Q_{r, t}^{T \max }+Q_{r, t}^{S}\right) \\
O H_{r, t}^{H C}=\frac{P E_{r, t-1}+P E_{r, t}}{2}-T W_{r, t}^{H C}
\end{gathered}
$$


Equation 2.7 represents a linear interpolation based on the highest turbine release value for each operating head in the plant power table. $T W_{r, t}^{H C}$ is the tailwater elevation used in the hydro capacity calculation. It is a function of a tailwater base value, $T W_{r, t}^{B}$, which in the case of the model we use here is the pool elevation of the downstream reservoir, and the total outflow from the reservoir, $Q_{r, t}^{T \max }+Q_{r, t}^{S}$. The specific calculation of $T W_{r, t}^{H C}$ depends on the tailwater method selected by the user (see Section 2.3.5). $Q_{r, t}^{S}$ is the spill from the reservoir. The operating head for the hydro capacity calculation is then the difference between the pool elevation (forebay elevation), which is calculated as the mean of the ending elevations from the previous time step and the current time step, and the tailwater elevation from equation 2.8. This operating head is then used to update equation 2.7 for the iterative calculation. In the optimization solution, equations 2.6 to 2.8 are replaced by piecewise linear approximations based on approximation points set by the user.

If incremental reserve capacity is actually deployed to generate energy, then this quantity of energy should be removed from the reserve capacity to avoid double counting of the reserves. For example, if the system is carrying $100 \mathrm{MW}$ of reserve capacity for regulation up, and it is called on to actually generate $25 \mathrm{MW}$ of regulation up energy to balance an increase in load, then the regulation up (non-generating) capacity should be reduced to $75 \mathrm{MW}$. If the regulation up reserve capacity remains at $100 \mathrm{MW}$ while adding the $25 \mathrm{MW}$ of regulation up deployment energy, then this would represent carrying $125 \mathrm{MW}$ of regulation up capacity and would overestimate the burden of the reserve requirements on the system. For ancillary services that are deployed on time scales shorter than the one-hour time step used in our model, such as regulation, the deployment of the reserve cannot be modeled directly. Instead a deployment fraction is used to represent the fraction of the reserve that is deployed in actual generation on average over the time step. A deployment fraction is used for regulation up, regulation down, spinning reserve and non-spinning reserve. A deployment fraction is not used for load following. ${ }^{17}$ The total generation at a given time step is then the "scheduled" power plus the power associated with the deployment of ancillary services.

\footnotetext{
${ }^{17}$ Load following is treated differently in our model. Instead of modeling a deployment fraction, the load following reserve requirement is removed in the operations run thus releasing the reserve capacity set aside in the scheduling run to be used as necessary.
} 
Note that the deployment of regulation down is a decrease in generation. An expression can then be written for the scheduled power at each time step.

$$
P_{r, t}^{s c h e d}=P_{r, t}-R U_{r, t}^{D F} R U_{r, t}+R D_{r, t}^{D F} R D_{r, t}-S R_{r, t}^{D F} S R_{r, t}-N R_{r, t}^{D F} N R_{r, t}
$$

In equation $2.10, P_{r, t}$ is the total power generation at reservoir $r$ at time $t . P_{r, t}^{s c h e d}$ is the "scheduled" generation without ancillary services deployment. $R U_{r, t}^{D F}$ is the regulation up deployment fraction at reservoir $r$ and time $t$ (a user input value at each time step), and thus the combined term, $R U_{r, t}^{D F} R U_{r, t}$, is the average power associated with regulation up deployment over time step $t$; likewise for the remaining ancillary services.

The constraints for reserve capacity (equations 2.3 and 2.4) are then rewritten as follows to account for the deployment energy:

$$
\begin{gathered}
P_{r, t}^{s c h e d}+R U_{r, t}+S R_{r, t}+N R_{r, t}+L F_{r, t} \leq H C_{r, t} \\
P_{r, t}^{s c h e d}-R D r, t-L F_{r, t} \geq 0
\end{gathered}
$$

An alternative form for writing these constraints is

$$
\begin{gathered}
P_{r, t}+\left(1-R U_{r, t}^{D F}\right) R U_{r, t}+\left(1-S R_{r, t}^{D F}\right) S R_{r, t}+\left(1-N R_{r, t}^{D F}\right) N R_{r, t}+L F_{r, t}+R D_{r, t}^{D F} R D_{r, t} \leq H C_{r, t} \\
\quad P_{r, t}-\left(1-R D_{r, t}^{D F}\right) R D_{r, t}-L F_{r, t}-R U_{r, t}^{D F} R U_{r, t}-S R_{r, t}^{D F} S R_{r, t}-N R_{r, t}^{D F} N R_{r, t} \geq 0
\end{gathered}
$$

Each ancillary service product is then aggregated over all power reservoirs in the system to give total system capacity, $X_{S, t}$, for each type of reserve at time $t$. Here the subscript $S$ refers to a system-wide total quantity as opposed to the reserve quantity for an individual reservoir.

$$
X_{S, t}=\sum_{r=1}^{R} X_{r, t}, \forall X \in\{R U, R D, L F, S R, N R\}
$$

A system-wide requirement term, $X_{S, t}^{r e q}$, is added for each ancillary service with the value at each time step set by the user. This allows for the following constraint to be included in the 
operational policy: ${ }^{18}$

$$
X_{S, t} \geq X_{S, t}^{r e q}, \forall X \in\{R U, R D, L F, S R, N R\}, \forall t \in T
$$

\subsubsection{Economic Modeling}

Economic modeling includes the value of energy and ancillary services (regulation up, regulation down, spinning reserve and non-spinning reserve) sold into the market. Only energy and ancillary services in excess of those required to meet local load and reserve requirements can be sold into the market. Two new variables are introduced to model the economic value of energy: energy sell, $E_{S, t}^{\text {sell }}$, and unmet load, $U_{S, t}^{L}$. Again the subscript $S$ refers to a system-wide total quantity.

$$
\begin{aligned}
& E_{S, t}^{\text {sell }}= \begin{cases}E_{S, t}^{\text {sched }}-L_{S, t}^{\text {net },} & E_{S, t}^{\text {sched }}>L_{S, t}^{\text {net }} \\
0, & E_{S, t}^{\text {sched }} \leq L_{S, t}^{\text {net }}\end{cases} \\
& U_{S, t}^{L}= \begin{cases}L_{S, t}^{n e t}-E_{S, t}^{s c h e d}, & E_{S, t}^{\text {sched }} \leq L_{S, t}^{n e t} \\
0, & E_{S, t}^{s c h e d}>L_{S, t}^{n e t}\end{cases}
\end{aligned}
$$

$L_{S, t}^{n e t}$ is the net system load (load minus wind generation) in units of energy and is an input value at each time step. Theoretically net system load can be positive or negative (if wind generation is greater than the local load). In order to maintain compatibility with existing RiverWare methods, negative load values are set to zero. ${ }^{19}$ The remaining terms in equations 2.17 and 2.18 are variables in the optimization solution. $E_{S, t}^{\text {sched }}$ is the scheduled power from all reservoirs multiplied by the time step length (one hour here) to convert to units of energy.

$$
E_{S, t}^{s c h e d}=\sum_{r=1}^{R}\left(P_{r, t}^{s c h e d} \cdot 1 h r\right)
$$

\footnotetext{
${ }^{18}$ While equation 2.16 is the conceptual form of this constraint, the actual formulation in the model follows equation 2.31 in order to properly incorporate it into the economic modeling of ancillary services based on equation 2.27 .

${ }^{19}$ In our test case, negative loads occur for a limited number of time steps in the scheduling run for the highest wind penetration scenarios. This results in a somewhat more conservative (lower) energy bid than if the full negative load were included. In the operations run, once the committed day-ahead energy is included in the load, the net load is positive, and the wind generation simply acts to reduce the hydro capacity that must be used to meet the load. Effectively the wind energy introduced at these negative load time steps gets shifted into additional hydro capacity that can be used for generation at a later time step, and thus the overall impact of setting negative load values to zero on the final results is minimal.
} 
In the optimization solution this is defined as

$$
L_{S, t}^{n e t}+E_{S, t}^{s e l l}-U_{S, t}^{L} \leq E_{S, t}^{s c h e d}
$$

The variables $E_{S, t}^{\text {sell }}, E_{S, t}^{\text {sched }}$ and $U_{S, t}^{L}$ are constrained in the formulation of the optimization problem to be non-negative. If this non-negative constraint were not already included, we would write a soft policy constraint

$$
U_{S, t}^{L}=0, \forall t \in T
$$

Because the non-negativity constraint is already included, the soft policy constraint to meet the load is written as

$$
U_{S, t}^{L} \leq 0, \forall t \in T
$$

The total value of energy sold at a given time step is based on a piecewise linear function. For each additional block of energy sold, the unit value $(\$ / \mathrm{MWh})$ decreases to represent the effect that bidding additional energy with a low marginal cost (e.g. wind energy or hydropower) has on reducing the market clearing price. The total energy sold at each time step is the sum of the energy in each block.

$$
E_{S, t}^{\text {sell }}=\sum_{b=1}^{N_{b}} E_{b, t}^{\text {sell }}
$$

where $b$ is the block number, $N_{b}$ is the total number of blocks of energy, and $E_{b, t}^{\text {sell }}$ is the total energy sold in block $b$ at time $t$. The unit price of each block of energy at each time step, $P R_{b, t}^{E}$ $(\$ / \mathrm{MWh})$, is input by the user. The value of energy, $V_{S, t}^{E}$, for the total system at time $t$ is then calculated as

$$
V_{S, t}^{E}=\sum_{b=1}^{N_{b}}\left(P R_{b, t}^{E} E_{b, t}^{s e l l}\right)
$$

The modeling of ancillary services capacity sold into the market is similar to that of energy sold into the market.

$$
X_{S, t}^{\text {sell }}= \begin{cases}X_{S, t}-X_{S, t}^{r e q}, & X_{S, t}>X_{S, t}^{r e q} \\ 0, & X_{S, t} \leq X_{S, t}^{r e q}\end{cases}
$$




$$
\begin{aligned}
& U_{S, t}^{X}= \begin{cases}X_{S, t}^{r e q}-X_{S, t}, & X_{S, t} \leq X_{S, t}^{r e q} \\
0, & X_{S, t}>X_{S, t}^{r e q}\end{cases} \\
& X \in\{R U, R D, S R\}
\end{aligned}
$$

The $X_{S, t}^{r e q}$ terms are analogous to the load term in the energy formulation and are user inputs. The remaining terms are optimization variables. The following constraint is written in the optimization formulation:

$$
X_{S, t}^{r e q}+X_{S, t}^{\text {sell }}-U_{S, t}^{X} \leq X_{S, t}
$$

The formulation for non-spinning reserve is slightly different. This modification is to account for the fact that, while non-spinning reserve is one of the products in the CAISO market, the NERC reserve requirements are set for contingency reserve, $C R_{S, t}$, (the combined total of spinning and non-spinning reserve) and spinning reserve as a required fraction of the total contingency reserve. A requirement is not set directly for non-spinning reserve (see Section 2.4.3.5).

$$
\begin{gathered}
C R_{S, t}=S R_{S, t}+N R_{S, t} \\
N R_{S, t}^{\text {sell }}= \begin{cases}C R_{S, t}-C R_{S, t}^{r e q}-S R_{S, t}^{\text {sell }}, & C R_{S, t}-S R_{S, t}^{\text {sell }}>C R_{S, t}^{r e q} \\
0, & C R_{S, t}-S R_{S, t}^{\text {sell }} \leq C R_{S, t}^{r e q}\end{cases} \\
U_{S, t}^{C R}= \begin{cases}C R_{S, t}^{r e q}-C R_{S, t}, & C R_{S, t} \leq C R_{S, t}^{r e q} \\
0, & C R_{S, t}>C R_{S, t}^{r e q}\end{cases}
\end{gathered}
$$

Once again the non-negativity constraint applies for the optimization variables, so the following soft constraint to meet the reserve requirements is included in the policy set: ${ }^{20}$

$$
U_{S, t}^{X} \leq 0, \forall X \in\{R U, R D, S R, C R\}, \forall t \in T
$$

The value for ancillary services includes not only the capacity that is sold into the market but also the compensation for energy that is generated when deploying the ancillary services.

$$
V_{S, t}^{X}=\left(\sum_{b=1}^{N_{b}} P R_{b, t}^{X} X_{b, t}^{s e l l}\right)+P R_{t}^{X, D e p l} X_{S, t}^{D F} X_{S, t}^{\text {sell }}, X \in\{R U, S R, N R\}
$$

\footnotetext{
${ }^{20}$ Note that the system requirement here is based on contingency reserve, $C R$ and not on non-spinning reserve in accordance with NERC regulations.
} 
$V_{S, t}^{X}$ is the total value for the ancillary service, $X$, at time $t$. The term in parenthesis is analogous to the value term for energy (equation 2.24) but for the relevant ancillary service product (capacity only). The remaining term represents the value of the deployment energy. $P R_{t}^{X, D e p l}$ is the unit value $(\$ / \mathrm{MWh})$ of deployment energy for ancillary service $X$ at time $t$, and $X_{S, t}^{D F}$ is the deployment fraction, both user inputs. (See Sections 2.4.3.4and 2.4.3.6 for details on how these values are set in the test case we model in this study.) $X_{S, t}^{\text {sell }}$ is the amount of reserve capacity sold into the market for the given ancillary service. For regulation down, deployment is a reduction in generation and thus corresponds to a decrease in revenue, but the hydropower system is still compensated for the regulation down capacity, and the water remains in the reservoirs to utilize for generation at a later time.

$$
V_{S, t}^{R D}=\left(\sum_{b=1}^{N_{b}} P R_{b, t}^{R D} R D_{b, t}^{\text {sell }}\right)-C_{t}^{R D, D e p l} R D_{S, t}^{D F} R D_{S, t}^{\text {sell }}
$$

$C_{t}^{R D, D e p l}$ is the unit cost $(\$ / \mathrm{MWh})$ of regulation down deployment, similar to $P R_{t}^{X, D e p l}$ in equation 2.32. The total value for ancillary services at time $t$ is then the sum of the total value of each individual ancillary service.

$$
V_{S, t}^{A S}=V_{S, t}^{R D}+V_{S, t}^{R U}+V_{S, t}^{S R}+V_{S, t}^{N R}
$$

Note that, for each ancillary service, a single deployment fraction is used for the entire system, so it is assumed that the deployment fraction for the local reserve requirement and for the reserve capacity sold into the market for a given ancillary service will be the same at time $t$. (In general the deployment fraction varies with time.) The assumption that the deployment fraction is the same for the market and local reserves is a simplification and may not always be true, particularly when the market is within a different BA than the hydropower system. Also, our model assumes that the deployment fraction is the same for all reservoirs. This is also a simplification. In actual practice, AGC deploys ancillary services across a BA based on real-time conditions and individual generator characteristics. In the absence of specific data regarding AGC deployment of ancillary services at individual generating units, deploying each ancillary service in quantities proportional to the scheduled capacity is taken as a reasonable approximation, particularly when averaged over 
a one-hour time step. In addition, the deployment energy is a relatively small fraction of total generation. ${ }^{21}$ Shifting this already small fraction by a small amount between reservoirs would have relatively little impact on the model results. Sensitivity to the deployment of ancillary services and the unit value of deployment energy are not evaluated here. These are areas that could be explored in future research.

The energy in storage at the end of the run is also given an economic value. The energy in storage at a given reservoir, $E I S_{r, t}$, is a measure of the generating potential of the water stored in that reservoir as it passes through that project as well as all remaining downstream projects. It is the product of the storage at that reservoir, $S_{r, t}$, and the sum of the average power coefficients, $\overline{P C}_{r}$, for all downstream reservoirs. The power coefficient represents the energy generation per each unit of water that passes through the plant. (For notation purposes, the reservoirs are assumed to be numbered in order from upstream to downstream; therefore, the reservoir with subscript $r+1$ is the reservoir downstream from reservoir $r$. Likewise the reservoir with subscript $r-1$ is the reservoir upstream of reservoir $r$.)

$$
E I S_{r, t}=S_{r, t} \sum_{d=r}^{R} \overline{P C}_{r}
$$

For the post-optimization simulation, $\overline{P C}_{r}$ is simply calculated as the average of the power coefficient over all time steps in the simulation. For the optimization run, this must be approximated at the beginning of the run. It is done so based on initial pool elevations at the given reservoir and the downstream reservoir, $P E_{r, 0}$ and $P E_{r+1,0}$ respectively, and an estimated turbine release, ${ }^{22}$ $Q_{r}^{T, e s t}$, which are then used to calculate estimated tailwater, $T W_{r}^{e s t}$, operating head, $O H_{r}^{e s t}$, and power, $P_{r}^{e s t}$, as shown in equations 2.36 to 2.39 . The tailwater and power calculations are based on linear interpolations from the tailwater table and plant power table respectively.

$$
T W_{r}^{e s t}=P E_{r+1,0}+f\left(Q_{r}^{T, e s t}\right)
$$

\footnotetext{
${ }^{21}$ In the test case we model, ancillary services deployment energy is typically less than $2 \%$ of total hydro generation and never exceeds the combined minimum generation requirement from all reservoirs.

${ }^{22}$ An estimated turbine release of $80 \mathrm{kcfs}$ is used at all reservoirs in our test case for the power coefficient approximation.
} 


$$
\begin{gathered}
O H_{r}^{e s t}=P E_{r, 0}-T W_{r}^{e s t} \\
P_{r}^{e s t}=f\left(O H_{r}^{e s t}, Q_{r}^{T, e s t}\right) \\
\overline{P C}_{r}^{\text {opt }}=\frac{P_{r}^{e s t}}{Q_{r}^{T, e s t}}
\end{gathered}
$$

The ending EIS is multiplied by a unit price for energy in storage, $P R_{t}^{E I S}$ (a user input), to give the ending energy in storage value, $V_{S, \text { final }}^{\text {EIS }}$.

$$
V_{S, \text { final }}^{E I S}=P R_{\text {final }}^{E I S} \sum_{r=1}^{R} E I S_{r, \text { final }}
$$

\subsubsection{Other Physical Processes}

Additional physical processes modeled include the reservoir mass balance

$$
S_{r, t}=S_{r, t-1}+\left(Q_{r, t}^{\text {in }}-Q_{r, t}^{\text {out }}\right) \cdot 1 h r
$$

where $S_{r, t}$ is the storage at the end of time step $t$, and $Q_{r, t}^{i n}$ and $Q_{r, t}^{\text {out }}$ are the average total inflow and outflow during time step $t$. The total outflow is comprised of the turbine release and spill.

$$
Q_{r, t}^{\text {out }}=Q_{r, t}^{T}+Q_{r, t}^{S}
$$

All spill in the system is modeled as regulated spill. The spill flow rate, $Q_{r, t}^{S}$, is not dependent on reservoir pool elevation as would be the case if modeling an unregulated spillway. Flood conditions are not being modeled in our study, and it is assumed that all of the reservoirs have spill gates sufficient to pass normal flows even in the high flow season. Storage values for each reservoir are converted to pool elevations by linear interpolation from the user-input elevation-volume table (see Section 2.4.1). Additional losses and gains, such as evaporation, unregulated hydrologic inflows, seepage and diversions, are considered to be negligible in our study and are not included, though the functionality exists in RiverWare to include these processes within the proposed modeling framework.

Routing in RiverWare is incorporated through a reach object between reservoirs. Time-lag routing is used in our model. Generally the routing from one reservoir, $r-1$, to the next reservoir, 
$r$, is defined by

$$
Q_{r, t}^{i n}=Q_{r-1, t-\text { lagtime }}^{\text {out }}
$$

When the lag time does not fall evenly on a time step interval and assuming a one-hour time step, the routing becomes

$$
Q_{r, t}^{\text {in }}=(1-\alpha) Q_{r-1, t-l a g^{\prime}}^{\text {out }}+\alpha Q_{r-1, t-l a g^{\prime}-1}^{\text {out }}
$$

where $l a g^{\prime}$ is the lag time in hours rounded down to the next lowest integer value and

$$
\alpha=\text { lagtime }-l a g^{\prime}
$$

The tailwater method in RiverWare utilized for our study is TWbaseValuePlusLookupTable. This method begins with a tailwater base value, which can be linked to another object in the model (in the case of our model, the pool elevation of the next reservoir downstream). An additional tailwater elevation value is then added to the base value and is a function of the reservoir outflow. This value is linearly interpolated from a user-input tailwater table (see Section 2.4.1 and Table $2.2)$.

$$
T W_{r, t}=T W_{r, t}^{B}+f\left(Q_{r, t}^{\text {out }}\right)
$$

\section{$2.4 \quad$ Test System}

\subsubsection{Physical System Description}

The described methodology is applied to a test case run-of-river hydropower system representative of systems in the Pacific Northwest. In order to provide a realistic model, physical characteristics of the hydropower facilities are based on the physical characteristics of actual hydropower projects. While the system characteristics are similar enough to existing facilities to provide realism, sufficient modifications have been introduced in both the physical characteristics and the operating policy so that specific modeling results shown here should not be used to infer conclusions about the specific response of any particular system to wind integration.

The test system includes five hydropower reservoirs on a single river. A single inflow to the system is modeled. Additional tributaries or local hydrologic inflows are not included, though this 
capability does exist within the RiverWare modeling framework. The lag time between all reservoirs is assumed to be one hour. The cascading reservoir system has limited storage, approximately two days of typical flow, but the size of the individual reservoirs varies significantly (see Table 2.1) which has implications for how they must be operated. For example, Reservoir 1D, the third power reservoir in the system, has very little storage and thus very limited flexibility in its operations. It is therefore operated essentially as a run-of-river hydropower plant. For this reason, it is assumed to not have AGC capabilities and is excluded from providing ancillary services. All ancillary services, therefore, must be provided by the remaining four hydropower projects. The limited amount of storage has another important implication for modeling realistic policy in the system. Much of the non-power policy is specific to the final reservoir in the system which must re-regulate the flows passing through the hydropower system; however, because the system has such limited storage and operates much like a run-of-river system, the policy constraints on the final reservoir directly impact flow requirements and operations throughout the system.

A storage reservoir is upstream of the hydropower system and is the source of inflows to the hydropower system. In the scenarios we model here, the storage reservoir is assumed to not be owned or operated by the hydropower utility, and its operations are not optimized together with the coordinated hydropower system. This represents realistic operating conditions where operators of run-of-river hydropower systems must often manage flows from upstream reservoirs and hydropower plants that are not coordinated with their own. A screenshot of the RiverWare model workspace showing the hydropower system model is presented in Figure 2.4.

Reservoir elevation-storage relationships are all based on a quadratic function.

$$
\text { Storage }=a_{0}+a_{1} P E+a_{2} P E^{2}
$$

$P E$ is the pool elevation in feet, and the storage is in 1000 acre-feet. Parameter values and other reservoir characteristics are shown in Table 2.1.

The reservoirs in the system are assumed to be level reservoirs and are assumed to be contiguous. The backwater from one reservoir reaches the dam of the upstream reservoir; therefore the 


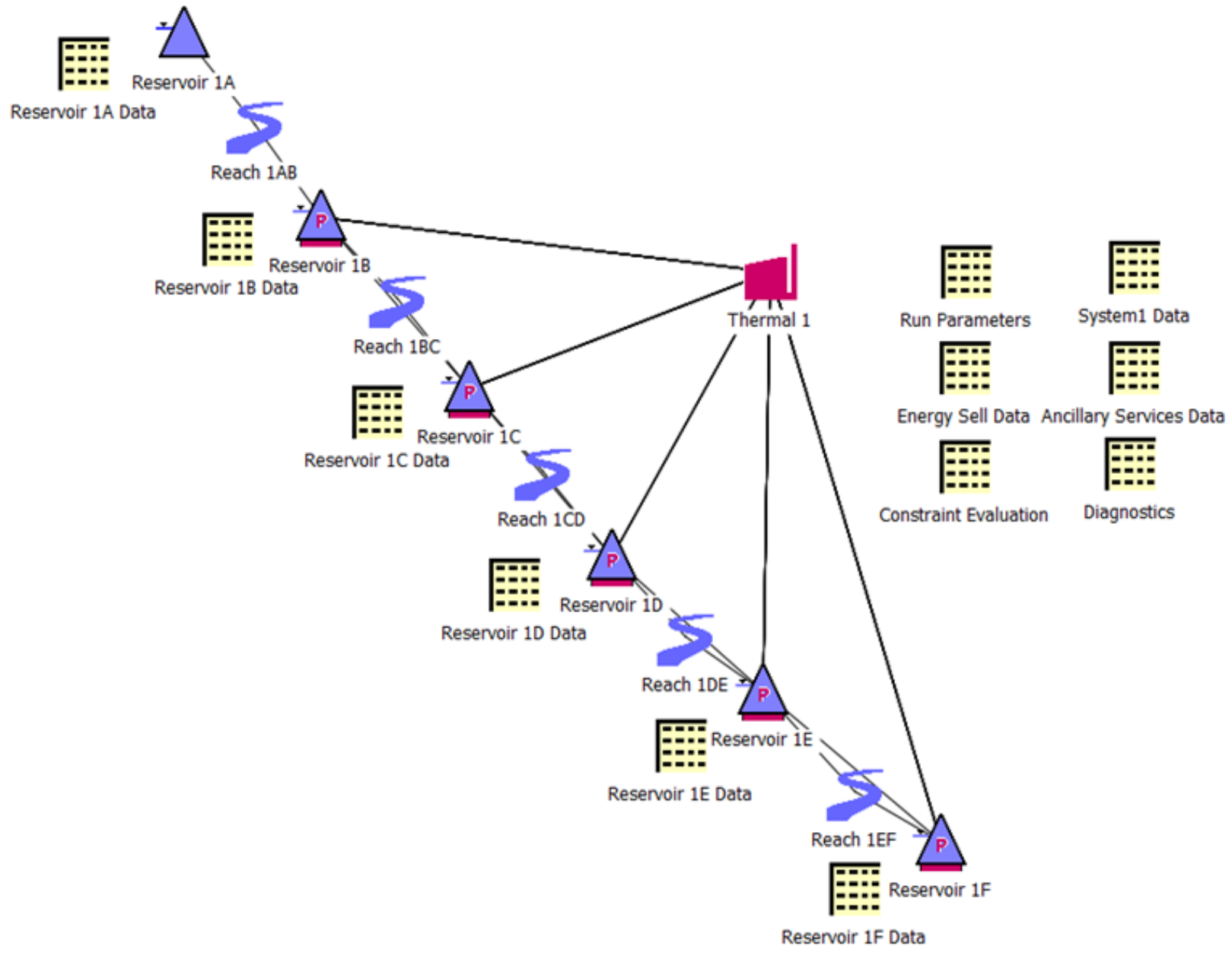

Figure 2.4: RiverWare model workspace showing 5 hydropower projects in a cascading reservoir system; Reservoir $1 \mathrm{~A}$ is a storage reservoir and is included here only to provide the inflows to the hydropower system.

Table 2.1: Reservoir storage and elevation-volume parameters

\begin{tabular}{cccrrrr}
\hline & Min Pool & Max Pool & & & & $\begin{array}{r}\text { Max Storage } \\
\text { Reservoir }\end{array}$ \\
Elev (ft) & Elev (ft) & $a_{0}$ & $a_{1}$ & $a_{2}$ & (1000-ft) \\
\hline 1A & 748 & 830 & 85406.64 & 275 & 0.215 & 5270.14 \\
1B & 484 & 495 & 46434.96 & 200 & 0.215 & 115.34 \\
1C & 416 & 420 & -1347.84 & 3 & 0.015 & 38.16 \\
1D & 321 & 325 & 540.88 & 605 & 0.015 & 12.76 \\
1E & 273 & 285 & 1607.97 & 25 & 0.070 & 168.72 \\
1F & 192 & 200 & 3056.64 & 28 & 0.115 & 56.64 \\
\hline
\end{tabular}


tailwater base value (equation 2.46) is the pool elevation of the downstream reservoir. The same tailwater curve is used for all reservoirs in the system and is shown in Figure 2.5 and in tabular form in Table 2.2. The tailwater value interpolated from the tailwater table is added to the downstream pool elevation to establish the tailwater elevation. For the final downstream reservoir, the tailwater base value is constant, and the tailwater elevation is simply a function of the outflow.

Table 2.2: Tailwater table: Tailwater values here are added to the tailwater base value.

\begin{tabular}{rr||rr}
\hline $\begin{array}{r}\text { Outflow } \\
(1000 \mathrm{cfs})\end{array}$ & $\begin{array}{r}\text { Tailwater } \\
\text { Value }(\mathrm{ft})\end{array}$ & $\begin{array}{r}\text { Outflow } \\
(1000 \mathrm{cfs})\end{array}$ & $\begin{array}{r}\text { Tailwater } \\
\text { Value }(\mathrm{ft})\end{array}$ \\
\hline 0 & 0.00 & 100 & 4.10 \\
10 & 0.00 & 120 & 5.30 \\
20 & 0.20 & 150 & 7.00 \\
30 & 0.40 & 200 & 9.60 \\
40 & 0.80 & 250 & 12.00 \\
50 & 1.20 & 300 & 14.25 \\
70 & 2.20 & 400 & 18.25 \\
\hline
\end{tabular}

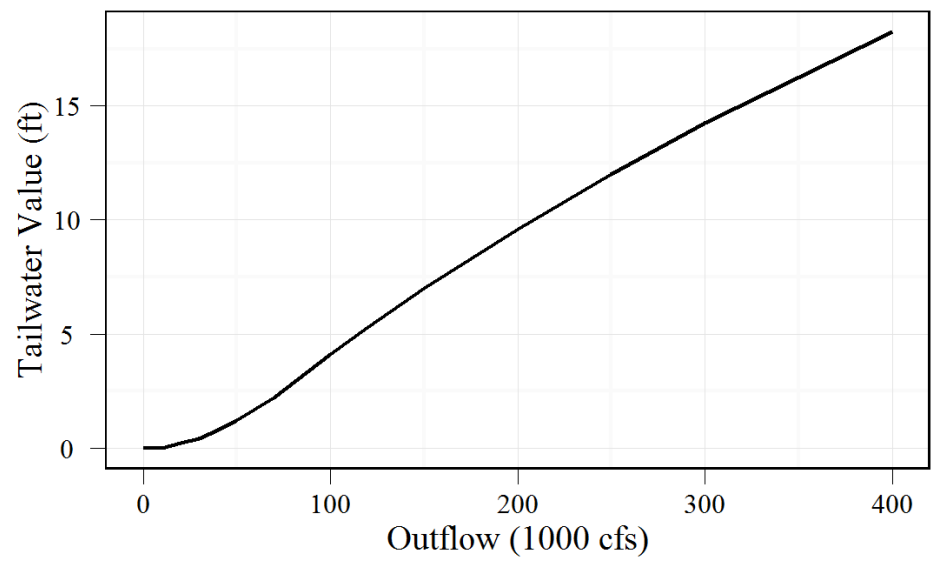

Figure 2.5: Tailwater curve used for all reservoirs

The points in the plant power table used to establish the piecewise linear power curve for each plant at each operating head are based on the power calculation.

$$
P=\eta \gamma H Q_{T}
$$


where $\eta$ is the overall plant efficiency, $\gamma$ is the specific weight of water, $H$ is the head and $Q_{T}$ is the turbine release. The efficiency is assumed to be a function of operating head and turbine release and has the form

$$
\eta=a_{0}+a_{1} H+a_{2} Q_{T}
$$

For each operating head in the plant power table, three turbine release and power points are set: zero flow, best efficiency and maximum capacity. A summary of plant power characteristics is shown in Table 2.3. Complete plant power tables for each reservoir are shown in Appendix A.

Table 2.3: Plant power charateristics

\begin{tabular}{|c|c|c|c|}
\hline Reservoir & $\begin{array}{l}\text { Best Efficiency } \\
\text { Flow (1000 cfs) }\end{array}$ & $\begin{array}{r}\text { Max Turbine } \\
\text { Capacity (1000 cfs) }\end{array}$ & $\begin{array}{r}\text { Max Generation } \\
\text { Capcity (MW) }\end{array}$ \\
\hline $1 \mathrm{~B}$ & 123.5 & 200 & 1016 \\
\hline $1 \mathrm{C}$ & 115.0 & 200 & 1286 \\
\hline $1 \mathrm{D}$ & 123.5 & 200 & 660 \\
\hline $1 \mathrm{E}$ & 112.3 & 180 & 1065 \\
\hline \multirow[t]{2}{*}{$1 \mathrm{~F}$} & 115.0 & 200 & 1164 \\
\hline & & Total Capacity & 5191 \\
\hline
\end{tabular}

\subsubsection{Policy}

Operational policies, in particular non-power constraints, are specific to each hydropower system and are dependent on the primary and secondary non-power purposes for which the dams may have been built (e.g. flood control, water storage and delivery, navigation). The policy implemented in our model is not intended to represent the actual policy of any single hydropower system. Rather it is designed to represent a sample, by no means exhaustive, of realistic power and non-power constraints that must be met by actual hydropower systems. The policies modeled here are most representative of policy constraints in the Pacific Northwest. Constraints are based on policies such as those coming from biological opinions and other agreements, such as the Hanford Reach Fall Chinook Protection Program Executed Agreement (2004), in place primarily to improve fish survival rates in the hydropower system. The general order of the policy priorities is given 
below. Note that all physical constraints that are part of standard RiverWare physical process algorithms (e.g. mass balance) are automatically included as hard constraints. The constraints described below are those included in the policy set designed specifically for this system.

- Licensing constraints (minimum and maximum pool elevations) and other imposed physical constraints (transmission)

- Non-power policy constraints (environmental flows)

- System power requirements (minimum generation, meeting local load, local reserve requirements)

- Lower priority non-power constraints

- Economic objective

The following is a description of each of the policy constraints and objectives given in their priority order. Some constraints are grouped together here though in the model, they are at separate priority levels. Also, additional constraints are included in the model policy set to improve the behavior of the optimization solution which are not included here. Only those which reflect actual operational policy are included.

\subsubsection{Licensing and Transmission Constraints}

License minimum and maximum pool elevations: These represent FERC ${ }^{23}$ licensing requirements and should never be violated.

$$
\begin{aligned}
& P E_{r, t} \geq P E_{r}^{\text {min }} \\
& P E_{r, t} \leq P E_{r}^{\text {max }} \\
& \forall r \in R, \forall t \in T
\end{aligned}
$$

${ }^{23}$ Federal Energy Regulatory Commission 
Transmission limits: This represents the transmission constraint between the hydropower system and the market BA and effectively limits the amount of energy and ancillary services that can be sold into the market. It is assumed that there are no transmission constraints within the hydropower system's BA. The transmission constraint is modeled as a static value throughout the run.

$$
E_{S, t}^{\text {sell }}+\left(R U_{S, t}^{\text {sell }}+S R_{S, t}^{\text {sell }}+N R_{S, t}^{\text {sell }}\right) \cdot 1 h r \leq E_{S, t}^{\text {MaxTrans }}, \forall t \in T
$$

For scenarios which include a real-time energy market, the real-time energy sold is included in this constraint as well. For base scenarios, the value of $E_{S, t}^{M a x T r a n s}$ is set to $1500 \mathrm{MW}$ for all $t$. Alternative scenarios evaluate the sensitivity to this value.

\subsubsection{Non-power Constraints}

Minimum outflows: This constraint sets the highest priority minimum outflow at all times at all reservoirs for environmental purposes. Other seasonal outflow constraints are more restrictive.

$$
Q_{r, t}^{\text {out }} \geq Q_{r}^{\min }, \forall r \in R, \forall t \in T
$$

The value of $Q_{r}^{\min }$ is $10 \mathrm{kcfs}$ for all but the final reservoir, $1 \mathrm{~F}$, where it is $40 \mathrm{kcfs}$.

Minimum spill for fish passage: Releasing water through the spillways can improve the survival of juvenile fish migrating downstream by allowing them to pass through the spillways as opposed to through the turbines. During late spring and summer, minimum spill is required to improve fish survival.

$$
Q_{r, t}^{S} \geq Q_{r, t}^{S \min }, \forall r \in R, \forall t \in \text { SpillSeason }
$$

Minimum seasonal environmental flows at Reservoir 1F: A number of the environmental constraints are specific to Reservoir $1 \mathrm{~F}$, which is the final reservoir to regulate flows after water passes through the hydropower system. During specific seasons, the minimum outflow from this reservoir may be required to be higher for environmental purposes such as fish survival (see 
the Hanford Reach Agreement).

$$
Q_{F, t}^{o u t} \geq Q_{F, t}^{E n v}, \forall t \in \text { EnvironmentalFlowSeasons }
$$

Maximum spill for total dissolved gas: High levels of spill can result in total dissolved gas (TDG) at supersaturation levels in the water. High TDG concentrations can result in significant fish mortality; therefore in hydropower systems that must deal with potentially lethal levels of TDGs, spill is managed to maintain TDG concentrations within specified limits (Bonneville Power Administration et al., 2009). The management of TDG levels is modeled in our test case in a simplified manner by using a maximum spill limit as a proxy for maximum allowable TDG levels.

$$
Q_{r, t}^{S} \leq Q_{r, t}^{S \max }, \forall r \in R, \forall t \in T
$$

Maximum daily outflow fluctuations at Reservoir 1F: This constraint which limits the amount by which the outflow at the final reservoir can change within a calendar day is based on restrictions on flows in the Hanford Reach Agreement to improve survival of juvenile salmon.

$$
Q_{F}^{\text {DailyMax }}-Q_{F}^{\text {DailyMin }} \leq \Delta Q_{F}^{\text {DailyMax }}
$$

Spawning season daylight flow restrictions: This constraint limits the outflow at Reservoir $1 \mathrm{~F}$ to a narrow range $(55-70 \mathrm{kcfs})$ from 6:00 a.m. to 6:00 p.m. in portions of October and November. It is based on spawning period flow restrictions in the Hanford Reach Agreement which are in place to assure that spawning salmon construct their redds (nests) and lay their eggs at appropriate levels in the river bed to improve their survival.

$$
\begin{aligned}
& Q_{F, t}^{\text {out }} \geq Q_{F}^{\text {SpawnMin }} \\
& Q_{F, t}^{\text {out }} \leq Q_{F}^{\text {SpawnMax }} \\
& 6: 00 \leq t \leq 18: 00
\end{aligned}
$$

Maximum hourly tailwater delta at Reservoir 1F: The change in the tailwater elevation at the final reservoir across one hour is constrained to be less than a maximum value in 
order to maintain favorable environmental conditions.

$$
\left|T W_{F, t}-T W_{F, t-1}\right| \leq \Delta T W_{F}^{\max }, \forall t \in T
$$

\subsubsection{Power-related Constraints}

Minimum generation: Each hydropower project in the system is required to maintain a minimum generation level. This is typically required to provide station service. Scheduled power is the variable used in the constraint to prevent the optimization solution from utilizing ancillary services deployment energy to meet the constraint.

$$
P_{r, t}^{s c h e d} \geq P_{r}^{\text {min }}, \forall r \in R, \forall t \in T
$$

Target/Scheduled ending storage: This constraint serves both power-related and nonpower purposes. In the scheduling run, this constraint prevents the optimization solution from draining the reservoirs to maximize revenue. In the operations run, this constraint forces the system to try to meet the storage levels set in the scheduling run within a tolerance. The target storage at the end of the one-week scheduling run is the same for all runs. The scheduled ending storage at the end of each six-hour operations run is one of the outputs from the scheduling run that is passed to the operations run. The tolerance term allows for a certain level of flexibility in the hydropower system to be utilized to meet changing conditions.

$$
\begin{gathered}
S_{r, f \text { final }}^{S C H}=S_{r}^{\text {target }}, \forall r \in R \\
S_{r, \text { final }}^{O P S} \geq S_{r, 6 h r}^{S C H}-S_{r}^{\text {tol }}, \forall r \in R
\end{gathered}
$$

Reserve restrictions due to other constraints: This set of constraints accounts for the fact that policy constraints limit the reserve capacity at each hydropower reservoir to a greater extent than the physical constraints alone. It further restricts decremental reserves due to minimum generation requirements, a modification of equation 2.4, and it restricts incremental reserves at Reservoir $1 \mathrm{~F}$ due to the higher priority flow constraints. Also, because the operations at Reservoir 
$1 \mathrm{~F}$ are significantly restricted due to flow constraints, it is constrained to carry no more than a specified percentage of the total system quantity of any single reserve.

$$
\begin{gathered}
R D_{r, t}+L F_{r, t} \leq P_{r, t}^{s c h e d}-P_{r}^{m i n}, \forall r \in R, \forall t \in T \\
R U_{F, t}+S R_{F, t}+N R_{F, t}+L F_{F, t} \leq H C_{F, t}^{\prime}-P_{F, t}^{s c h e d}, \forall t \in T \\
X_{F, t} \leq F_{F}^{\max } X_{t}, \forall X \in\{R U, R D, L F, S R, N R\}, \forall t \in T
\end{gathered}
$$

$H C_{F, t}^{\prime}$ is the effective hydro capacity at Reservoir $1 \mathrm{~F}$ when considering all non-power constraints. It is a function of the most restrictive maximum flow constraint that is in place at time $t . F_{F}^{\max }$ is the maximum fraction of any ancillary service that can be carried by Reservoir 1F.

Meet reserve requirements: In the scheduling run the reserve requirement represents the quantity of each ancillary service product the hydropower system must provide within its own BA. In the operations run, any ancillary services that are committed in the day-ahead market during the scheduling run are included in the reserve requirement for this constraint as well. In the policy set, this is actually written as a constraint on the Unmet Reserve Requirement. Requiring the unmet quantity to be zero is the equivalent of meeting the requirement (see equation 2.26). This is done so that the economic evaluation is formulated properly (equation 2.27).

$$
U_{S, t}^{X} \leq 0, \forall X \in\{R U, R D, L F, S R, C R\}, \forall t \in T
$$

Note that contingency reserve, $C R$, is used in this constraint in place of non-spinning reserve. Contingency reserve is simply the combined total of spinning reserve and non-spinning reserve. This is in accordance with WECC policy which places requirements on the total contingency reserve within a $\mathrm{BA}$ and then requires that a certain percentage of that total contingency reserve come from spinning reserve (WECC, 2008). See Section 2.4.3 for a description of how each of the reserve requirements are calculated.

Meet load: Hydropower generation is required to be greater than or equal to the net load. In the scheduling run, the net load includes only the net within the BA. Additional generation beyond the net load is assumed to be bid into the day-ahead energy market. For the operations 
run, energy committed in the day-ahead market is also included in the net load. Like the constraints for reserve requirements, this is actually written as a constraint on unmet load (see equation 2.20).

$$
U_{S, t}^{L} \leq 0, \forall t \in T
$$

Scheduled power and reserves: Additional soft constraints are added for each power reservoir only in the operations run to try to force the system to meet total system energy and reserve requirements by using the scheduled energy and reserves at each hydropower plant as nearly as possible. This is to prevent the system from completely re-optimizing during actual operations with perfect forecast knowledge and is intended to capture the loss of efficiency that can occur when operations must be adjusted from the optimized schedule to meet changing real-time conditions. Generation is allowed to adjust at each plant within the limits of the balancing reserves that are scheduled at that plant. ${ }^{24}$ Any deviations from this schedule will be minimized by the repeated minimax solution.

$$
\begin{gathered}
P_{r, t}^{O P S} \geq P_{r, t}^{S C H}-\left(L F_{r, t}^{S C H}+R D_{r, t}^{S C H}\right), \forall r \in R, \forall t \in T \\
Q_{r, t}^{T, O P S} \leq Q_{r, t}^{T, S C H}+\frac{L F_{r, t}^{S C H}+R U_{r, t}^{S C H}}{\overline{P C}_{r}^{o p t}}, \forall r \in R, \forall t \in T
\end{gathered}
$$

The less than or equal to constraint must be based on turbine release instead of power directly in order to maintain convexity in the optimization solution. The constraints for scheduled reserves are similar.

$$
\begin{gathered}
X_{r, t}^{O P S} \geq X_{r, t}^{S C H}-X_{t o l}, \forall r \in R, \forall t \in T \\
X_{r, t}^{O P S} \leq X_{r, t}^{S C H}+X_{t o l}, \forall r \in R, \forall t \in T \\
\forall X \in\{R U, R D, S R, N R\}
\end{gathered}
$$

\footnotetext{
${ }^{24}$ Note that regulation up and down are included in the balancing reserves in this constraint only to represent a small amount additional flexibility beyond the scheduled load following reserves. At times the necessary adjustments cannot be met by scheduled load following reserves alone. The system can utilize additional capacity up to the amount of the regulation reserves at a given reservoir if additional capacity is available after meeting the initial load and reserve requirements. It is not, however, using any of the actual regulation capacity set to meet the reserve requirement in equation 2.67 .
} 
The ancillary services tolerance value, $X_{t o l}$, is a user input and is set to $3 \mathrm{MW}$ for all ancillary services in our model. There is no load following requirement in the operations run to represent the use of scheduled load following reserve to cover any net load forecast error.

\subsubsection{Lower priority non-power constraints}

Target recreation levels at Reservoirs 1B and 1E: A lower priority non-power constraint is included to maintain specified reservoir levels in the two largest reservoirs for recreational purposes. In this case the recreation constraints are at a priority below the power constraints. It is noted that in many systems, constraints on recreational pool elevations or recreational flows may take precedence over power objectives for specific seasons.

$$
P E_{r, t} \geq P E_{r, t}^{R e c}, \forall t \in T, \forall r \in\{1 B, 1 E\}
$$

\subsubsection{Economic objective}

At the final priority is the economic objective. For the scheduling run this includes maximizing the combined total value of energy and ancillary services sold in the day-ahead market. For the operations run, the objective is to maximize the value of energy in storage at the end of each six-hour period. This objective does have the effect of trying to keep as much water upstream as possible in order to place the hydropower system in the best position for the following run; however, the system is already constrained to meet minimum scheduled ending storage values at each reservoir (see equation 2.63), so the EIS objective has a limited effect on the solution in our test case model.

Scheduling objective: Maximize combined energy and ancillary services value

$$
\text { Maximize }: V_{\text {Total }}=\sum_{t=1}^{T}\left(V_{S, t}^{E}+V_{S, t}^{A S}\right)
$$

Operations objective: Maximize ending energy in storage value

$$
\text { Maximize }: V_{S, \text { final }}^{E I S}=P R_{\text {final }}^{E I S} \sum_{r=1}^{R} E I S_{r, \text { final }}
$$


In select scenarios, a real-time market is included in the operations run. In the real-time market scenarios, the objective for the operations run is to maximize the value of energy in the real-time market. A real-time ancillary services market is not considered in our analysis.

\section{Alternative operations objective: Maximize real-time energy value}

$$
\text { Maximize }: V_{\text {Total }}^{R T}=\sum_{t=1}^{T} V_{S, t}^{E, R T}
$$

\subsubsection{Input Data}

In order to create realistic scenarios, all model inputs for load, hydrology, wind and prices come from observed time-synchronized data sets from select weeks in $2010 .^{25}$ Milligan et al. (2011) discuss the importance of using time synchronized data for wind integration studies. This allows for correlations between factors such as weather conditions, hydrologic conditions, load and energy prices to be accounted for. A description of all input data sources for our test case is given here as well as how any data are manipulated for use in the model.

\subsubsection{Load and Load Forecast Data}

Load data are based on Mid-Columbia hourly aggregated non-federal request data. It is important to note that the Mid-Columbia request data are not a 'true load' but rather a combination of generation requests from a set of utilities that use utilize their share of the Mid-Columbia system to meet a portion of their own load. One result of this is that the Mid-Columbia raw data display greater intra-hour variability than typical load for a BA, so a five hour rolling average is applied to smooth the data. Also, the data tend to be more highly correlated with hydrologic conditions than would be the case for most loads. This can be considered a reasonable approximation, however, of a utility which owns a mix hydro and thermal generating sources and shifts more of its load to the hydropower system during periods of high flows and more towards the thermal system in periods of lower flows. The correlation of load with hydrologic conditions is also explored in the sensitivity

\footnotetext{
${ }^{25}$ There are exceptions to this in certain select alternative scenarios where some input data are intentionally not time-synchronized in order to evaluate the sensitivity to factors such as the timing of wind generation in relation to prices.
} 
analysis by scaling the load for different scenarios. The base scenarios use $85 \%$ of the smoothed Mid-Columbia requests as the local load for the hydropower system. Table 2.4 shows a summary of the local load data for the three Base scenarios assuming a maximum hydropower generation value of $4000 \mathrm{MW}$.

Table 2.4: Load input data for base scenarios

\begin{tabular}{rrrr}
\hline Average Load & $\begin{array}{r}\text { Average Load } \\
\text { (MW) }\end{array}$ & $\begin{array}{r}\text { Peak Load Max Gen }) \\
(\text { MW })\end{array}$ \\
\hline Spring & 2225 & 55.6 & 2833 \\
Summer & 2814 & 70.4 & 3019 \\
Fall & 1693 & 42.3 & 2395 \\
\hline
\end{tabular}

Synthetic load forecast error data (without wind) are generated for the day-ahead forecast using an $\operatorname{ARMA}(2,1)$ model for the percent error. The forecast error models for the two, three and four day ahead forecasts use AR(1) models that also include cross-correlation terms for the forecast percent error from the same hour and the previous hour in the previous day's forecast. All lead times beyond four days use the four day forecast. The models for each lead time are fit independently based on an assumed mean absolute percent error. The MAPE for the day-ahead forecast is $2.5 \%$, a typical value for day-ahead load forecast error (Taylor and McSharry, 2007), and increases as the lead time increases. A similar model is developed by de Mello et al. (2011) to generate synthetic load and wind forecasts. In that work, however, cross-correlations are between real-time, hour-ahead and day-ahead forecasts. Also, all parameters are jointly optimized for all lead times to match standard deviation, autocorrelation and cross-correlation statistics. The load forecast models used in our study are described in equations 2.77 to 2.79 . In these equations $\epsilon_{t}^{L d}$ is the load forecast percent error at time $t$ at a lead time of $d$ in days, $\alpha_{1}^{d}$ and $\alpha_{2}^{d}$ are the fitted lag-1 and lag-2 auto-regressive coefficients for forecast lead time $d, \beta_{1}^{d}$ is the fitted moving average coefficient, and $\gamma_{1}$ and $\gamma_{2}$ are the fitted cross-correlation coefficients. $L_{S, t}^{F d}$ is the system load forecast (MW) at time $t$ for lead time $d$ in days, and $L_{S, t}$ is the actual system load (before subtracting wind generation) at time $t$. The parameter values for the load forecast error models 
are shown in Table 2.5.

Day-ahead load forecast percent error:

$$
\epsilon_{t}^{L 1}=\alpha_{1}^{1} \epsilon_{t-1}^{L 1}+\alpha_{2}^{1} \epsilon_{t-2}^{L 1}+N_{t}^{L 1}\left(0, \sigma^{L 1}\right)+\beta_{1}^{1} N_{t-1}^{1}\left(0, \sigma^{L 1}\right)
$$

Two, three and four day ahead load forecast percent error:

$$
\begin{gathered}
\epsilon_{t}^{L d}=\alpha_{1}^{d} \epsilon_{t-1}^{L d}+N_{t}^{L d}\left(0, \sigma^{L d}\right)+\gamma_{1}^{L d} \epsilon_{t}^{L d-1}+\gamma_{2}^{L d} \epsilon_{t-1}^{L d-2}, d \in\{2,3,4\} \\
L_{S, t}^{F d}=\epsilon_{t}^{L d} L_{S, t}
\end{gathered}
$$

Table 2.5: Load forecast model parameters

\begin{tabular}{rrrrr}
\hline Lead Time & 1 Day & 2 Days & 3 Days & $4-7$ Days \\
\hline$\alpha_{1}$ & 0.439 & 0.254 & 0.162 & 0.158 \\
$\alpha_{2}$ & 0.099 & $\mathrm{NA}$ & $\mathrm{NA}$ & $\mathrm{NA}$ \\
$\beta_{1}$ & 0.736 & $\mathrm{NA}$ & $\mathrm{NA}$ & $\mathrm{NA}$ \\
$\gamma_{1}$ & $\mathrm{NA}$ & 0.656 & 0.391 & 0.376 \\
$\gamma_{2}$ & $\mathrm{NA}$ & 0.141 & 0.629 & 0.588 \\
$\sigma$ & 0.015 & 0.025 & 0.008 & 0.008 \\
$\mathrm{MAPE}$ & 0.025 & 0.030 & 0.0325 & 0.035 \\
\hline
\end{tabular}

The magnitude of the load forecast error is relatively small, particularly when compared to the wind generation forecast error. While the general distribution of load forecast error is significant because it contributes to the reserve requirement calculation (see Section 2.4.3.5), the hydropower model is relatively insensitive to the specific trace of load forecast error. Only a single trace of load forecast error is used, therefore, for each scenario whereas multiple traces of wind forecast error are modeled. It is also important to note that it is the net load forecast (load forecast less wind forecast) that is used as an input to the model, so within the model there is no distinction as to whether a change in the net load forecast comes from the load forecast or the wind forecast.

\subsubsection{Hydrologic Data}

Inflow data for the hydropower system (modeled as outflow from Reservoir 1A) are taken from Grand Coulee Dam hourly outflow data. ${ }^{26}$ This represents the fact that operators of run-of-

\footnotetext{
${ }^{26}$ http://www.nwd-wc.usace.army.mil/ftppub/water_quality/10data/
} 
river hydropower systems often have to manage variable flows resulting from releases from upstream hydropower dams. A perfect hydrologic forecast is assumed, however, for the one-week planning horizon.

\subsubsection{Wind Data}

Wind generation inputs for the model are based on scaling observed wind generation data from Bonneville Power Administration. ${ }^{27}$ Synthetic wind generation forecasts are produced using an $\operatorname{ARMA}(2,1)$ model and a Box-Cox transformation of the forecast error for the day-ahead forecast. For two-day-ahead and three-day-ahead forecasts, additional error is added by separate ARMA models. The three-day-ahead forecast is used for all lead times of three to seven days. Chapter 3 provides a detailed description of the of the wind generation data and wind forecast model used for this study. Multiple traces of wind generation forecasts are generated for each actual wind generation time series. All other inputs to the model remain constant when running the multiple wind generation forecast traces for a given scenario.

Four different wind penetration levels are modeled for each scenario: $10 \%, 20 \%, 30 \%$ and 40\%, which correspond to installed wind capacities of 444 MW, 1000 MW, 1714 MW and 2667 MW respectively. The $40 \%$ wind penetration case is recognized as a relatively extreme penetration scenario and is included in our study only to evaluate system behavior at the upper limits of wind integration.

\subsubsection{Price Data}

The initial market clearing prices for energy and ancillary services (regulation down, regulation up, spinning reserve, and non-spinning reserve) are taken from observed CAISO MCPs. ${ }^{28} 29$ Day-ahead hourly prices correspond to the same time periods in 2010 as the raw load, wind and hydrologic data. For the scenarios which include a real-time energy market, the average hourly

\footnotetext{
${ }^{27}$ http://transmission.bpa.gov/Business/Operations/Wind/default.aspx

28 http://www.caiso.com/market/Pages/ReportsBulletins/ReportsBulletinsArchive/Default.aspx

29 http://oasis.caiso.com/mrtu-oasis $/$ ?doframe=true\&serverurl=http $\% 3 a \% 2 f \% 2 f f r p t p 09 \% 2$ eoa $\% 2$ ecaiso $\% 2$ ecom $\% 3$ a $8000 \&$ volume $=$ OASIS
} 
real-time MCPs from the same dates and hours in 2008 are used. ${ }^{30}$ These initial data are set as the block 1 prices $\left(P R_{1, t}^{E}\right.$ see Section 2.3.4). Then each block of additional energy (or ancillary service capacity) sold reduces the market clearing price by a fixed percentage (a parameter value). In energy markets, all energy generation bids that are awarded are compensated at the same market clearing price. In order to simulate this, $P R_{b, t}^{E}$, which represents the unit price $(\$ / \mathrm{MWh})$ of each new block $b$ of energy sold at time $t$, is reduced such that the average price across all used blocks decreases by this fixed percentage. $R^{E}$ represents the reduction factor, the fixed percentage by which the MCP decreases for each block.

$$
\frac{\sum_{c=1}^{b} P R_{c, t}^{E}}{b}=\left(1-R^{E}\right) \frac{\sum_{c=1}^{b-1} P R_{c, t}^{E}}{b-1}
$$

For example, if the initial unit price is $\$ 100 / \mathrm{MWh}$, and the MCP is assumed to decrease $1 \%$ for each additional block of energy, then the unit price for the second block would be $\$ 98 / \mathrm{MWh}$ so that the average across both blocks is $\$ 99 / \mathrm{MWh}$. This is defined by the following equation:

$$
P R_{b, t}^{E}=\max \left(0.01, P R_{1, t}^{E}\left(1-R^{E}\right)^{b-2}\left(1-b R^{E}\right)\right), b \geq 2
$$

The lower limit for the price of a given block is set at $\$ 0.01 / \mathrm{MWh}$ to prevent the optimization solution from using the linear piecewise power segments out of order if the price were zero or negative. In reality energy prices can become negative, primarily in the real-time market when there is an excess of energy in the system. It can be assumed that if the model is selling energy at $\$ 0.01 / \mathrm{MWh}$, it represents a situation in which the hydropower system is being forced to produce power at negative prices due to physical and/or policy constraints. A sample enregy value curve and corresponding price curve are shown in Figure 2.6.

For energy, the prices are set for 50 MWh blocks. For day-ahead energy, the modeled MCP decreases by $1 \%$ for each additional $50 \mathrm{MWh}$ sold $\left(R^{E}=0.01\right) \cdot{ }^{31}$ The real-time market is assumed to have less depth than the day-ahead market, and thus the reduction factor is set to $5 \%$ and

\footnotetext{
${ }^{30}$ http://oasishis.caiso.com/index.jsp

31 This is based on an a simplification and approximation of results observed by Jónsson et al. (2010) when evaluating the impact of wind generation on energy prices in the Nord Pools Elspot market.
} 
(a)

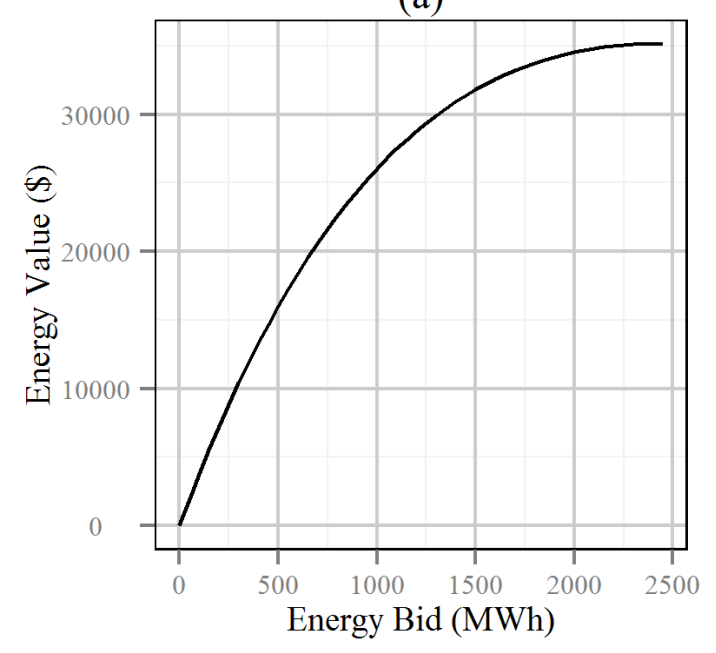

(b)

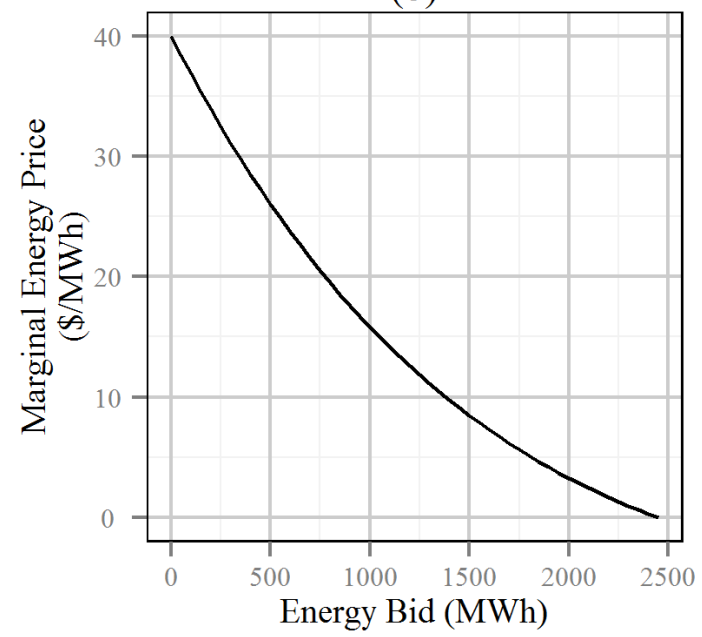

Figure 2.6: (a) Sample energy value curve and (b) corresponding price curve representing the market depth for energy with an initial (block 1) MCP of $\$ 40 / \mathrm{MWh}$ and reduction factor $\left(R^{E}\right)$ of $1 \% / 50 \mathrm{MWh}$. Plot (b) represents the curve described by equation 2.81 and is the derivative of (a).

$10 \%$ per $50 \mathrm{MWh}$ for different scenarios. The prices for ancillary services are based on $10 \mathrm{MW}$ blocks, and the reduction factors are $4 \%$ per $10 \mathrm{MW}$ for regulation down and 5\% per $10 \mathrm{MW}$ for regulation up, spinning reserve, and non-spinning reserve. It is recognized that the shape of the price curve for a given product can vary considerably from one market to another dependent on a number of factors, particularly the mix of available generation resources and their associated marginal costs. The parameter values we use here are only intended as a simplified example of the effect of increased generation on market prices. The sensitivity to the price curve parameter for the day-ahead energy market is tested by evaluating alternative scenarios in which this parameter value is varied, including a scenario which assumes unlimited market depth (no change in prices with increased generation).

The unit value of ancillary services deployment energy for regulation up, spinning reserve and non-spinning reserve $\left(P R_{t}^{X, D e p l}\right.$ see Equation 2.32) is set equal to the price of the first block of real-time energy at time step $t$. For regulation down, the unit cost of deployment energy, $C_{t}^{R D, D e p l}$, is set equal to the price of the first block of day-ahead energy at time step $t$. The unit value of 
energy in storage, $P R_{t}^{E I S}$, is set equal to the daily average price of the first block of day-ahead energy. Note that the EIS unit value does not affect the optimization solution, but it does impact the relative contribution of the EIS value to the total system value. The total system value is evaluated in post-processing of the model outputs; therefore different assumptions about the value of $E I S$ can be tested by simply scaling the final $E I S$ value. ${ }^{32}$

\subsubsection{Reserve Requirements}

One of the primary effects of increased wind penetration on power systems is its impact on reserve requirements for the system. In our study, reserve requirements are calculated outside the model based on existing methods described below and imported as input data. There is no attempt to optimize the amount of local reserves within the model. (See Morales et al., 2009; Ortega-Vazquez and Kirschen, 2009; Leite da Silva et al., 2011, for alternative methods to establish reserve quantities which optimize their value in the system.)

Contingency Reserve: The contingency reserve requirement we use here is based on the WECC requirement that a reserve sharing group or BA that is not part of a reserve sharing group carry a contingency reserve that is equal to the greater of the largest single potential contingency in the BA, such as the sudden loss of the largest generating unit, or the sum of $3 \%$ of load and $3 \%$ of generation (WECC, 2008). For base scenarios the contingency reserve requirement in our model is set to $500 \mathrm{MW}$, which is assumed to represent the portion of the single largest contingency in the BA that must be covered by the hydropower system. ${ }^{33}$ Alternative scenarios also test contingency reserve requirements of $250 \mathrm{MW}$ and $1000 \mathrm{MW}$. The WECC standard also states that at least half of the required contingency reserve must be spinning reserve.

$$
S R_{S, t}^{r e q}=0.5 C R_{S, t}^{r e q}
$$

\footnotetext{
${ }^{32}$ While modifying the EIS unit value obviously changes the total system value, it was observed that this had relatively little impact on general trends and relative results between scenarios.

33 The $3 \%$ load plus $3 \%$ generation calculation typically results in a contingency reserve requirement under 200 MW for our model.
} 
Regulating Reserve: There are not formal regulatory requirements on regulating reserves. Rather NERC sets maximum allowable area control error (ACE) based on deviations from the standard frequency of $60 \mathrm{~Hz}$ (NERC, 2007). Each BA must then determine the amount of regulating reserves it should maintain in order to assure that it will meet the NERC standards. The WWSIS uses a regulation requirement of $1 \%$ of peak load within each transmission area (GE Energy, 2010). The EWITS assumes 1\% of total load to be approximately equal to three times the standard deviation of load variability at the regulating time scale and uses this as the basis for the regulation requirement. The variability of wind at this time scale is assumed to be negligible and thus does not impact the regulation requirement (EnerNex, 2010). The regulating reserve requirement we use here follows the EWITS approach of using $1 \%$ of load. ${ }^{34}$

$$
R U_{S, t}^{r e q}=R D_{S, t}^{r e q}=0.01 L_{S, t}
$$

Load Following Reserve: Load following reserve does not have an external requirement. The method we use in this study for determining the load following requirement is based on methods used in Acker et al. (2012b); Holttinen et al. (2008) and Robitaille et al. (2012). The total load following reserve requirement, $L F_{S, t}^{r e q}$, has two components. It is scheduled to assure that there is sufficient capacity, both up and down, to balance longer time scale variability in net load. It is also used in the day-ahead scheduling run to assure that there is sufficient capacity scheduled to cover net load forecast error.

For the load variability component, $L F_{N L \Delta}^{r e q}$, the requirement is based on the distribution of hourly net load changes $\left(L_{S, t}^{n e t}-L_{S, t-1}^{n e t}\right)$. The requirement is set equal to the absolute value of the hourly net load change corresponding to a set exceedence probability. ${ }^{35}$ We use an exceedence probability of 0.995 . The variability component of the load following requirement is evaluated for each calendar month.

\footnotetext{
${ }^{34}$ The EWITS regulating reserve requirement also includes a term for wind forecast error. In our study, all wind forecast error is assumed to be covered by load following reserve, and thus this additional term is not included in the regulation requirement.

${ }^{35}$ It is also common to use 10-minute or 15-minute net load changes. Only hourly load data are available here. Also some methodologies use a multiple of standard deviations, often 3 , in place of exceedence probability, but this can underestimate the magnitude of extreme events due to the non-Gaussian distribution (Acker et al., 2012b)
} 
Reserve Capacity for Net Load Variability:

$$
P\left(\left|L_{S, t}^{n e t}-L_{S, t-1}^{n e t}\right| \leq L F_{N L \Delta}^{r e q}\right)=0.995
$$

The forecast error component, $L F_{N L F E}^{r e q}$, is similar. In the absence of empirical forecast data, one hundred traces of day-ahead load and wind forecasts are generated for each month using the synthetic forecast generators described in Sections 3.2 and 2.4.3.1 and observed wind and load for that month. The load forecast error, $\epsilon_{t}^{L}$, and wind forecast error, $\epsilon_{t}^{W}$, (both in MW) are then calculated and combined into the net load forecast error. The reserve requirement is again calculated as the forecast error value (absolute) corresponding to an exceedence probability of 0.995 .

Reserve Capacity for Net Load Forecast Error:

$$
P\left(\left|\epsilon_{t}^{L}+\epsilon_{t}^{W}\right| \leq L F_{N L F E}^{r e q}\right)=0.995
$$

Assuming that these two components of load following are uncorrelated, the combined requirement is calculated by taking the square root of the sum of the squares of the individual requirements.

$$
L F_{S, t}^{r e q}=\sqrt{\left(L F_{N L \Delta}^{r e q}\right)^{2}+\left(L F_{N L F E}^{r e q}\right)^{2}}
$$

The load following reserve requirement is removed in the operations run to simulate the use of the reserve capacity to meet the actual load as it deviates from the forecast. The load following reserve requirement is a critical parameter value in terms of how it impacts the results of wind integration studies. The values used for load following reserve in our model are heavily influenced by the assumptions that generation is being scheduled based on day-ahead forecasts and at an hourly time step. In order to evaluate the sensitivity to these assumptions, alternative scenarios are run in which load following reserve requirment is reduced. These scenarios use exceedence probabilities of 0.99 and 0.98 when establishing the load following reserve requirement. This could also be taken as a proxy for modeling an improved wind forecast where the reduced forecast error results in a reduction in the forecast error component of the reserve requirement. 
A summary of the incremental reserve requirements for the spring base scenario is shown in Table 2.6. Total reserve requirements for the summer and fall scenarios are within $4 \%$ of these values. Additional methodologies for determining operating reserve requirements for wind integration studies are described in Holttinen et al. (2008) and Ela et al. (2010).

Table 2.6: Incremental reserve requirments, spring base scenario

\begin{tabular}{cr||rrr|r}
\hline Penetration & $\begin{array}{r}\text { Installed } \\
\text { Wind (MW) }\end{array}$ & $\begin{array}{r}\text { Regulation Up } \\
(\text { Avg MW) }\end{array}$ & $\begin{array}{r}\text { Contingency } \\
\text { Reserve (MW) }\end{array}$ & $\begin{array}{r}\text { Load Following } \\
\text { Reserve (MW) }\end{array}$ & $\begin{array}{r}\text { Total } \\
(\mathrm{MW})\end{array}$ \\
\hline No Wind & 0 & 22.2 & 500 & 303.3 & 825.6 \\
$10 \%$ & 444 & 22.2 & 500 & 435.9 & 958.1 \\
$20 \%$ & 1000 & 22.2 & 500 & 789.0 & 1311.2 \\
$30 \%$ & 1714 & 22.2 & 500 & 1290.9 & 1813.2 \\
$40 \%$ & 2667 & 22.2 & 500 & 1979.3 & 2501.5 \\
\hline
\end{tabular}

\subsubsection{Ancillary Services Deployment Energy}

Regulation is assumed to correspond to random fluctuations in net load, and thus over a onehour time step net regulation deployment energy should be approximately zero. When modeled as two separate reserve products, however, as we do here, the hourly average of each is non-zero. For the scheduling run, the regulation up and regulation down deployment fractions are set to 0.2 for all time steps representing the average percentage of regulation capacity that is expected to be deployed during operations. For the operations run, the deployment fraction is modeled as a random normal variable. ${ }^{36}$ Like other model parameters, regulation deployment can vary from one system to another. Only a single trace of regulation deployment is modeled for each scenario. The sensitivity to regulation deployment is not evaluated in our study.

Whereas regulation is being deployed constantly, the contingency reserve is assumed to be deployed much less frequently as it is only to cover major contingency events. When contingency reserves are deployed, it is typically only for a short time period (WECC, 2008). For the schedul-

\footnotetext{
36 Values for the mean and standard deviation are based on an analysis of regulation data from the PJM market for May4-8, 2009; available at http://pjm.com/markets-and-operations/ancillary-services/mkt-basedregulation/archived-mkt-based-regulation.aspx
} 
ing run the spinning reserve is assigned a deployment fraction of 0.02 , and non-spinning has a deployment fraction of 0.01 to represent how much the reserve is assumed to be deployed over the long-term for planning purposes. In the operations run, however, the deployment fraction for each is set to 0 . No contingency event occurs during the run period. The one exception is a single scenario that is run to test the system response to a large contingency event. In this case the deployment fraction for both spinning and non-spinning reserve is set to 0.9 for a single time step representing a $450 \mathrm{MW}$ loss of generation within the BA.

\subsubsection{Scenario Descriptions}

As stated previously, the impacts and value of integrated wind generation and hydropower are dependent on a large number of factors and can vary significantly with seasonal conditions. Many existing wind integrations studies run scenarios for a complete year or multiple years to evaluate the effects over a large range of conditions, often to arrive at an average cost of wind integration. Our approach here is to model specific weeks to evaluate the sensitivity of wind and hydro integration to changes in system conditions with variations in select system parameters. This allows the effects of specific seasonal conditions to be analyzed. Descriptions of the scenarios used to carry this out are given here.

\subsubsection{Base Scenarios: Full Policy}

Three weeks are selected which have different hydrologic and wind conditions as well as having different seasonal non-power constraints in effect. These are modeled with the full policy set in place for the Base scenarios.

\section{Spring Scenario: May 14-20, 2010}

The first scenario is intended to model relatively average stream flow. A minimum spill requirement for fish passage is in effect for the final three reservoirs ( $8 \mathrm{kcfs}$ at Reservoir 1D and 20 kcfs at Reservoirs $1 \mathrm{E}$ and $1 \mathrm{~F})$. A seasonal minimum environmental flow of $65 \mathrm{kcfs}$ is required at Reservoir $1 \mathrm{~F}$. The daily outflow fluctuation at Reservoir $1 \mathrm{~F}$ is limited to $20 \mathrm{kcfs}$. 


\section{Summer High Flow and High Wind Scenario: June 22-28, 2010}

The second scenario represents a high flow scenario with high wind generation, often one of the critical operating conditions for hydropower systems with integrated wind generation. The excess water flowing through the system can increase the need to run the hydropower plants as near to capacity as possible. This can reduce the flexibility for balancing wind generation. While this scenario is constrained by physical conditions, it is the least restricted by non-power policy constraints. In this scenario, minimum spill requirements are in effect for all five dams (15 kcfs at Reservoirs $1 \mathrm{~B}$ and 1C, $20 \mathrm{kcfs}$ at Reservoirs $1 \mathrm{D}$ and $1 \mathrm{E}$, and $25 \mathrm{kcfs}$ at Reservoir 1F), although the reservoirs tend to spill in excess of the minimum even without added wind generation due to the high flows in the system. The maximum allowable spill for TDG limits is increased to $100 \mathrm{kcfs}$ at each dam (75 kcfs at Reservoir 1D). This tends to be the most controlling constraint on the system in this scenario. The maximum allowable daily outflow fluctuation at Reservoir $1 \mathrm{~F}$ is increased to 40 kcfs.

\section{Fall Scenario: October 30-November 5, 2010}

The third scenario is marked by the restricted outflow range at Reservoir $1 \mathrm{~F}$ during daylight hours (55 kcfs-70 kcfs) to protect spawning salmon (based on spawning season requirements in the Hanford Reach Fall Chinook Protection Program Executed Agreement (2004)). This constraint

affects scheduling throughout the system as reservoirs must position themselves during nighttime hours to be able to meet the narrow flow requirements for the following day. This is also the most water limited scenario. There is no minimum spill requirement.

\subsubsection{Reduced Policy Scenarios}

In order to evaluate the impact of modeling non-power policy, the baseline scenarios are compared to the same scenarios with two levels of simplified policy sets. The only non-power constraints in effect for the Unconstrained case are minimum and maximum pool elevations, constant minimum outflows (10 kcfs) for all reservoirs and target ending storages. The Moderate Policy case adds minimum spill and minimum seasonal environmental flow requirements. All power-related 
constraints and objectives are in place for all policy cases. It is necessary to clarify that the reduced policy cases are included only to show the importance of accounting for non-power policy when modeling hydropower systems. They are not included to suggest alternative operating policy for the hydropower system.

\subsubsection{Effects of Wind Forecast Error, Wind Variability and Wind Energy}

The effects of adding wind generation to the power system are divided into three components: energy, variability and forecast error. The effects of the added energy are assumed to be positive in general (though in certain circumstances, particularly high-flow seasons, excess energy can create difficulties for the system). Added energy in the system means more water can be stored in the hydropower reservoirs and used for generation when most beneficial. It can also mean that more energy is available to sell for increased revenue. In general, over a long-term average, wind variability and forecast error are assumed to have negative impacts on the system. The negative impacts are a combination of the increased reserve requirements due to variability and forecast error (see Section 2.4.3.5) and the direct impacts such as the increased ramping and starts and stops from variability or the shifting of operations away from peak efficiency due to forecast error. In the short-term for specific scenarios, however, they may be observed to benefit the system, such as if the variability happens to match up with demand patterns or peak pricing.

In order to isolate the effects of these three components of integrated wind generation, within each scenario four separate cases are modeled: A No Wind case; a Full Wind case ${ }^{37}$ which includes wind generation in the net load including forecast error in the scheduling run; a No Error case ${ }^{38}$ which includes wind generation but uses a perfect wind forecast in the scheduling run; and a No Variability case which includes the equivalent amount of wind energy but without the variability and without forecast error. The net value from each isolated component of wind generation can

\footnotetext{
${ }^{37}$ Each case with wind is modeled at all four penetration levels.

38 No Error here refers to wind forecast error only. Load forecast error is still included in all cases.
} 
then be calculated as shown in equations 2.87 to 2.89 .

$$
\begin{gathered}
\text { NetWindForecastErrorValue }=\text { TotalValue }(\text { FullWind })-\text { TotalValue }(\text { NoError }) \\
\text { NetWindVariabilityValue }=\text { TotalValue }(\text { NoError })-\text { TotalValue }(\text { NoVariability }) \\
\text { NetWindEnergyValue }=\text { TotalValue }(\text { NoVariability })-\text { TotalValue }(\text { NoWind })
\end{gathered}
$$

Dividing each net value by the total wind generation at the given penetration gives the average value of each component per unit of wind generation. Other model outputs can be compared in the same manner. For example, any difference in spill between the Full Wind case and the No Error case can be assumed to be due to wind forecast error. For the No Variability case, rather than model the added wind energy as a constant flat block, the equivalent amount of wind energy over the one-week scenario is shaped to approximately follow the load with a ramping rate approximately equal to that of a coal plant. The energy resource ramps from $60 \%$ capacity during low load hours to $100 \%$ capacity over the hours 5:00 to $9: 00$ and then back down to $60 \%$ over the hours 18:00 to 22:00. A sample time series plot of the No Variability case compared to actual wind for the same scenario is shown in Figure 2.7.

\subsubsection{Alternative Scenarios for Sensitivity Analysis}

In addition to the scenarios described above, the sensitivity of the results to select modeling assumptions and parameter values is evaluated by running alternative scenarios described here. In general, these sensitivities are tested in the spring scenario only, unless specified otherwise.

Real-time market: Two scenarios include a real-time market in the operations run approximated by hourly energy prices. These scenarios are used to evaluate effects of the assumption that all generation is scheduled based on day-ahead forecasts. The availability of a real-time market allows additional capacity scheduled as reserves to be utilized to generate revenue once real-time conditions are known. The two scenarios differ only in their price curve parameters. In one the real-time energy price decreases $5 \%$ per $50 \mathrm{MWh}$ of energy sold. In the second the price decreases $10 \%$ per 50MWh. 


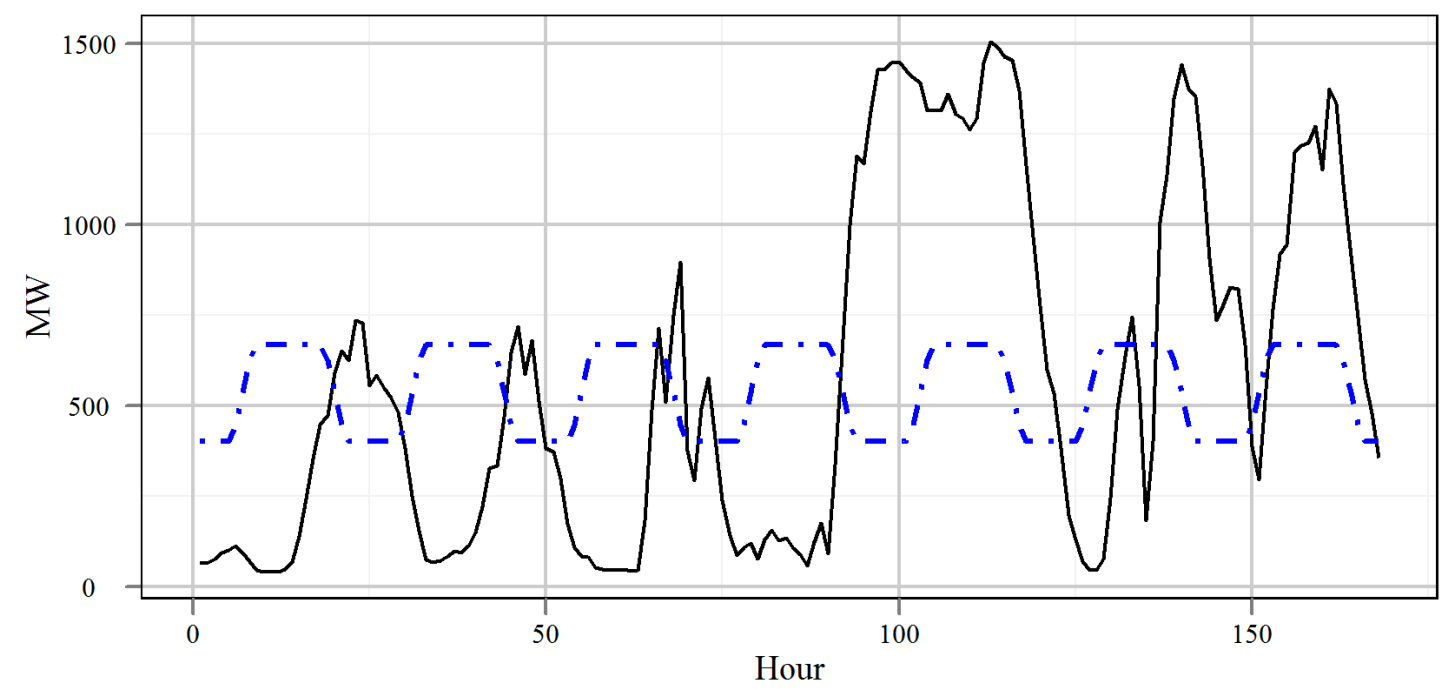

Figure 2.7: Time series of actual wind (solid line) and the corresponding No Variability wind (dashed line) for the same scenario at $30 \%$ penetration

Load following reserve requirement: Two alternative scenarios use exceedence probabilities of 0.98 and 0.99 to calculate the load following reserve requirement (see Section 2.4.3.5). (The Base case uses an exceedence probability of 0.995.) These scenarios test the sensitivity to a major assumption that all reserves are scheduled based on day-ahead forecast error characteristics. This can be interpreted as an approximation of either scheduling generation and reserves in a shorter time horizon or scheduling based on improved forecasts. The forecasts are not actually altered in these scenarios. Only the load following requirement is changed.

Load levels: High and low local load cases are modeled for both the spring and summer scenarios. The High Load case uses $100 \%$ of the smoothed Mid-Columbia request data. The Low Load case uses $50 \%$ of the smoothed Mid-Columbia request. A summary of the load inputs for these scenarios is shown in Table 2.7 .

Transmission constraints: Available transmission is one of the primary constraining factors for large scale wind integration (EnerNex, 2010). A scenario with the transmission limit raised to $2500 \mathrm{MW}$ (from the $1500 \mathrm{MW}$ Base case) is modeled for the spring and summer scenarios, 
Table 2.7: Load input data for alternative load level scenarios

\begin{tabular}{r||rrr|rrr|rrr}
\hline \multicolumn{1}{l||}{} & \multicolumn{3}{c|}{ Base Case } & \multicolumn{3}{c|}{ High Load Case } & \multicolumn{3}{c}{ Low Load Case } \\
\hline & $\begin{array}{r}\text { Avg } \\
\text { Scenario }\end{array}$ & $\begin{array}{r}\text { Avg } \\
(\text { MW }\end{array}$ & $\begin{array}{r}\text { Peak } \\
(\text { Max })\end{array}$ & $\begin{array}{r}\text { Avg } \\
(\text { MW })\end{array}$ & $\begin{array}{r}\text { Avg } \\
(\% \text { Max })\end{array}$ & $\begin{array}{r}\text { Peak } \\
(\text { MW })\end{array}$ & $\begin{array}{r}\text { Avg } \\
(\text { MW })\end{array}$ & $\begin{array}{r}\text { Avg } \\
(\% \text { Max })\end{array}$ & $\begin{array}{r}\text { Peak } \\
(\text { MW })\end{array}$ \\
\hline \hline Spring & 2225 & 55.6 & 2833 & 2617 & 65.4 & 3333 & 1309 & 32.7 & 1667 \\
Summer & 2814 & 70.4 & 3019 & 3311 & 82.8 & 3552 & 1655 & 41.4 & 1776 \\
\hline
\end{tabular}

and unlimited transmission is modeled for the summer scenario to evaluate the extent to which transmission becomes a critical constraining factor in this model.

Low load and increased transmission: The $2500 \mathrm{MW}$ and unlimited transmission cases are combined with the low load case for the spring and summer scenarios to further explore the sensitivity of the results to transmission limits, particularly when there is excess energy in the system.

Market depth: For a Low Market Depth case the day-ahead energy price is reduced by $2 \%$ per $50 \mathrm{MWh}$ (from the Base case of $1 \%$ per $50 \mathrm{MWh}$ ). An Unlimited Market Depth case is also modeled where the price at a given time step does not change regardless of the amount of energy sold.

Contingency reserve: The contingency reserve requirement is reduced to $250 \mathrm{MW}$ (from the Base case of $500 \mathrm{MW}$ ) for one scenario and raised to $1000 \mathrm{MW}$ for another scenario to test the sensitivity to the assumption about how much contingency reserve the hydropower system is required to carry.

Contingency event: A contingency event is modeled that represents the loss of a 450 MW generator in the BA at noon on the fifth day of the run, during a period when the wind is ramping down. This scenario is to evaluate the flexibility of the system to actually provide the necessary balancing generation. Placing the contingency event at the first time step of one of the six hour operations runs prevents any forecast knowledge of the event in the optimization solution so that the system cannot re-position itself to prepare for the contingency event. 
Wind timing: In order to test the effects of the wind timing in relation to load and energy prices, scenarios are run with the wind generation and corresponding forecast shifted back 6,12 and 18 hours. These scenarios intentionally violate the principle of maintaining time-synchronized data in order to specifically evaluate the sensitivity to the timing of inputs.

Summer hydrology with spring prices: The summer scenario is run with spring energy and ancillary services price inputs in order to evaluate the extent to which the changes in system behavior between the spring and summer scenarios are due to the different hydrologic and wind conditions and how much is simply due to different prices. Like the wind timing scenarios, this scenario intentionally violates the principle of maintaining time-synchronized data.

\subsection{Assumptions and Simplifications}

In addition to assumptions and simplifications that have been mentioned in the previous sections, it is important to identify the following assumptions that affect the model results as well as some points on what our methodology does not do.

- Operational costs for ancillary services, such as increased maintenance costs due to wear on equipment, are not included due to lack of sufficient data to model such costs. See Piekutowski et al. (2012) for a description of the sources of potential costs to hydropower resources from increased ancillary services use.

- The hydropower resources in our model are assumed to provide all of the reserves that are required from the integrated wind generation. In some systems, a portion of these reserves may come from thermal resources such as gas turbines.

- The wind generators are not assumed to make any payments for the ancillary services required to balance the wind variability and uncertainty. Some systems may have payment mechanisms in place for such services.

- There are no transmission constraints within the modeled utility. In reality there may be geographic constraints on reserve requirements within a BA related to transmission 
constraints that require certain levels of reserve capacity within specific geographic regions of the BA and not only within the BA as a whole.

- Hydropower unit outages are not considered.

- Each scenario run is deterministic. A single wind generation forecast corresponds to a single realized wind generation time series. Though multiple traces of wind forecast are modeled for each scenario, there is no stochastic optimization within the model.

- Our methodology does not directly model unit commitment or economic dispatch of other generating resources outside of the integrated hydropower and wind system. The effects of a different mix of generating resources in the system are assumed to be approximated by different load and market depth scenarios, though how these scenarios correspond to specific generation portfolios is not explored in depth here.

- Our approach does not make a comparison to wind integration without coordinated hydropower as a balancing reserve as is done in some integration studies (see, for example, Angarita and Usaola, 2007; Benitez et al., 2008; Matevosyan et al., 2009). Rather it uses hydropower without integrated wind generation as the Base case then assesses the net value of wind added to the hydropower system. It does not assess the value of hydropower for balancing wind. 


\section{Chapter 3}

\section{Wind Generation Modeling}

The response of a hydropower system to integrated wind generation depends in part on the amount of installed wind capacity and the total wind generation. It also depends on characteristics such as the variability in wind generation, wind forecasting methods and the resulting forecast error, and the timing of wind generation relative to peaks in load and energy prices. All of these characteristics, in addition to the installed wind capacity, can vary significantly for different geographic regions and for each integrated wind and hydropower system. The methodology we present here does not attempt to model hydropower and wind integration under all possible conditions and wind characteristics. Rather an attempt is made to model realistic scenarios of actual wind generation and wind forecast error recognizing that these represent only a small sample of possible wind generation scenarios and specific results could vary when evaluating wind integration in other systems. A sensitivity analysis is carried out to partially evaluate the effects of varying certain characteristics of the wind inputs. This includes shifting the timing of the wind generation and reducing the amount of reserve capacity scheduled to cover wind forecast errors which would accompany a reduction in wind forecast error. Details of this sensitivity analysis are discussed further in sections 2.4.4.4 and 4.5.3. 


\subsection{Actual Wind Generation Data}

The wind generation data used in this study are based on Bonneville Power Administration (BPA) Balancing Authority total wind generation in 2010. ${ }^{1}$ Numerous wind integration studies have used synthetic wind generation data for actual wind inputs as well as synthetic wind forecast inputs. In the WWSIS, the level of wind capacity to be modeled was well beyond current installed capacity, and thus wind generation time series inputs were simulated based on weather models and power characteristics of sets of wind turbines assumed to be located at selected wind farm locations (GE Energy, 2010). The BPA data are used here for several reasons. First it is a readily available set of actual generation data for an aggregated set of wind farms which contains true characteristics of wind generation. The BPA installed wind capacity from 2010 is approximately equal to the installed capacity for the $40 \%$ penetration case, the highest wind penetration case modeled in our study, and thus there is not a need to produce additional synthetic wind data. Also these wind generation data come from the same region as the hydrologic input data. It can be assumed, then, that correlations that may exist between hydrologic conditions and wind are being captured in these datasets. In addition, much of the BPA wind generation is used to meet California RPS requirements and can therefore have an impact on energy prices in the CAISO market. Using the BPA wind data and the CAISO price data as inputs to our model captures this correlation as well.

Wind penetration can be defined differently for different BAs. Often it is calculated as installed wind capacity as a percent of peak load. Like the BPA balancing area, the system we model here is a net exporter of energy. In this case it is more reasonable to define penetration in terms of installed wind as a percent of peak generation instead of peak load. In our model the peak generation is defined as the maximum hydropower generation plus the maximum wind capacity. The expression for wind penetration is then

$$
W_{p e n}=\frac{P_{p e n, \max }^{W}}{P_{p e n, \max }^{W}+P_{\max }^{H}}
$$

\footnotetext{
${ }^{1}$ http://transmission.bpa.gov/Business/Operations/Wind/default.aspx
} 
where $W_{p e n}$ is wind penetration, $P_{p e n, \max }^{W}$ is the installed wind capacity at the given penetration, and $P_{\max }^{H}$ is the maximum hydropower generation. (Note that the maximum hydropower generation is less than the nameplate capacity.) The installed wind capacity based on a set penetration level is then calculated as

$$
P_{p e n, \max }^{W}=\frac{P_{\max }^{H} W_{p e n}}{1-W_{p e n}}
$$

The modeled wind penetration cases are 10\%, 20\%,30\% and 40\%. The assumed maximum hydropower load is assumed to be $4000 \mathrm{MW}$. The corresponding installed wind capacity amounts are then 444 MW, $1000 \mathrm{MW}, 1714 \mathrm{MW}$ and 2667 MW respectively.

The BPA data are available in five minute intervals and are averaged to hourly values then scaled to represent the desired wind penetration levels used in our study. It is widely recognized that wind generation variability does not remain constant as the level of penetration changes. As increased wind capacity is brought online, the geographical distribution generally increases resulting in less correlation in outputs between individual wind generators; therefore the relative variability for large amounts of wind generation is less than for smaller amounts (Wan, 2004, 2005). In order to account for this it is necessary to scale the variability in the wind data in addition to scaling the mean. The total BPA wind capacity in 2010 was approximately equal to the $40 \%$ wind case in our model. It is therefore necessary to increase the variability from the scaled, observed data for the lower penetration scenarios in order to account for the reduced geographic distribution of wind generation resources associated with decreased installed capacity.

The BPA wind generation data are only available in aggregate, so there are no direct means by which to determine the effect on variability of removing portions of the wind resources and reducing the geographic distribution. As a substitute, to estimate a reasonable change in variability, wind data from select sites in the WWSIS dataset are used. ${ }^{2}$ The WWSIS dataset provides ten minute simulated wind data for sites in the western United States based on weather conditions for 2004, 2005, and 2006. Only the 2006 data are used in our analysis. WWSIS sites are selected such

\footnotetext{
2 The WWSIS dataset is provided by the National Renewable Energy Laboratory, which is operated by the Alliance for Sustainable Energy, LLC for the U.S. Department Of Energy and is available at http://www.nrel.gov/wind/integrationdatasets/western/data.html.
} 
that the total annual mean generation and the annual standard deviation of wind generation at ten minute intervals are approximately equal to the BPA 2010 mean and ten-minute standard deviation $\left(\sigma_{10}\right)$. Then sites are removed from the selected WWSIS sites so that the annual mean generation is equal to that of the $30 \%, 20 \%$, and $10 \%$ wind cases in our model. With each reduction the geographic spread of the wind sites is decreased which has the effect of increasing the relative variability. ${ }^{3}$ The four cases based on the WWSIS data are then converted to hourly wind data, and the annual 60-minute standard deviation $\left(\sigma_{60}^{p e n}\right)$ is calculated for each as well as the 60 -minute standard deviation for the BPA wind data $\left(\sigma_{60}^{B P A}\right)$. The ratio of the synthetic 60 -minute standard deviation to the BPA 60-minute standard deviation is then used to scale the deviation of wind generation from the scaled mean at each time step.

First the mean wind generation value, $\bar{P}_{\text {pen }}^{W}$, for the given penetration level pen is calculated from the installed capacity for that penetration level and an assumed capacity factor for wind, $F_{C}$, which is set to 0.3 , a typical capacity factor for wind generation.

$$
\bar{P}_{p e n}^{W}=P_{p e n, \max }^{W} F_{C}
$$

To calculate the "actual" wind generation, $P_{\text {pen,t }}^{W}$ at time $t$ a second term is added to the mean generation value which represents the scaled deviation from the mean and incorporates the scaled variability.

$$
P_{p e n, t}^{W}=\bar{P}_{p e n}^{W}+\frac{\sigma_{60}^{p e n}}{\sigma_{60}^{B P A}}\left(P_{B P A, t}^{W}-\bar{P}_{B P A}^{W}\right)
$$

$\sigma_{60}^{p e n}$ is the standard deviation of 60 -minute wind generation for penetration pen based on the $W W S I S$ data, and $\sigma_{60}^{B P A}$ is the BPA 60-minute standard deviation. $P_{B P A, t}^{W}$ is the observed BPA wind generation at time $t$, and $\bar{P}_{B P A}^{W}$ is the mean observed BPA wind generation. Appendix B contains a listing of the WWSIS sites used to estimate the standard deviation for each penetration case.

\footnotetext{
3 The relative variability can be measured by the coefficient of variation, the standard deviation divided by the mean: $C O V=\sigma / \mu$. Details from this variability analysis including the $W W S I S$ sites used and the resulting mean, standard deviation and $C O V$ values are shown in Appendix B.
} 


\subsection{Wind Generation Forecast}

The integrated hydropower and wind generation modeling methodology presented in Chapter 2 requires wind generation forecasts ranging from day-ahead to seven days ahead. Wind generation forecast data at these lead times are not available for the actual BPA wind generation data that are used as the basis for the wind generation inputs into our model. In the absence of real forecast data, synthetic wind generation forecasts are produced based on a statistical analysis of combined observed actual wind generation data and wind generation forecast data made available from a set of sample wind farms for the months of August through October. It is recognized that the characteristics of wind generation forecast error can vary from one system to another and may depend on a number of factors including installed wind capacity, geographic distribution of wind generation resources, regional weather patterns, season, and forecasting methodologies employed. The goal with the synthetic wind generation forecast methodology we present here is not to reproduce wind generation forecasts that match those that would have corresponded to the actual BPA wind generation data. Rather the purpose is to produce synthetic wind forecast time series, given an actual wind forecast time series, that provide a reasonable and realistic characterization of real wind generation forecast error recognizing that the specific characteristics of forecast error may be different for other systems. The analysis is carried out on the forecast percent error normalized by the installed wind capacity so that the statistical characteristics can be transferred to another system with a different level of installed capacity and different actual wind generation characteristics.

\subsubsection{Existing Statistical Wind Forecast Methodologies}

Statistically-based synthetic wind forecast models have been developed previously for use in wind integration studies. Two relevant examples, both of which use autoregressive moving average (ARMA) models, are described here. 
Soder (2004) develops an ARMA(1,1) model to produce synthetic wind speed forecast errors for a single wind generation site. An optimization routine is used to fit the ARMA model parameters with an objective of minimizing the squared difference between root mean squared error (RMSE) from the model and RMSE in observed forecasts summed over a range of lead times. This model is applied by Matevosyan et al. (2009) to develop a scenario tree of forecast errors, which when added to a given wind speed forecast, provides a scenario tree of possible wind speed outcomes. The wind speed forecast data are converted to wind generation forecasts based on modeled wind turbine characteristics. The wind forecast and scenario tree are subsequently used in a stochastic optimization algorithm to optimize the coordinated operation of a hydropower system and wind farm.

de Mello et al. (2011) present a similar $\operatorname{ARMA}(1,1)$ model for wind generation forecast errors with several key differences. They define three separate ARMA models each with their own parameter values for real-time (5 minutes ahead), hour-ahead and day-ahead wind generation forecast errors. The hour-ahead model includes a cross-correlation term which correlates the hourahead forecast error with the real-time error from the same time step. The day-ahead model contains a similar term to correlate the error with the hour-ahead model. An optimization algorithm then fits parameters for all three models simultaneously. The other key difference is that the objective for the fitting algorithm does not only minimize the differences between model and observed mean error values. Rather it minimizes a weighted combination of the differences in observed and model values for mean, standard deviation, auto-correlation and cross-correlation. In this manner, it attempts to capture more of the statistical characteristics of forecast error beyond the mean alone.

\subsubsection{Analysis of Observed Wind Generation Forecast Error}

The two ARMA models mentioned in section 3.2.1 assume that wind generation forecast errors are normally distributed with a mean of zero. Hodge and Milligan (2011) show that forecast errors in shorter time frames (five minutes to three hours) are not Gaussian but rather have a higher kurtosis than a normal distribution. In general, a normal distribution would underestimate the 
number of time periods with very small and very large forecast error, and it would over predict the number of time periods with moderate forecast error. de Mello et al. (2011) also mention that the real-time forecast error distribution has more small errors and fewer large errors (greater kurtosis) than a normal distribution and that the distributions for longer time scales are not symmetric, but all of the distributions are assumed to be approximately Gaussian when applying the forecast error model.

The actual wind generation data that have been provided from sample wind farms are at ten minute intervals. These ten-minute data have been averaged into hourly values. The corresponding wind generation forecast data are at fifteen minute intervals. At each interval a wind generation forecast is given for each hour from one hour out to 72 hours out. From these fifteen minute data, forecasts at the top of each hour have been extracted for lead times of 12, 24, 36, 48, 60 and 72 hours and are paired with the corresponding averaged hourly actual generation for that forecast hour. The actual generation is subtracted from the forecast at each hour and for each lead time to produce the forecast error. These error values are then normalize by dividing by the installed wind capacity to produce a time series of wind generation forecast error as a percent of installed capacity for each selected lead time. Histograms of the normalized forecast error at each lead time overlaid with fitted normal distributions are shown in Figure 3.1. Statistics of the normalized forecast error distributions for each lead time are displayed in Table 3.1.

Table 3.1: Statistics of normalized observed wind generation forecast error over a range of lead times

\begin{tabular}{rrrrrrr}
\hline Lead Time & $12 \mathrm{hr}$ & $24 \mathrm{hr}$ & $36 \mathrm{hr}$ & $48 \mathrm{hr}$ & $60 \mathrm{hr}$ & $72 \mathrm{hr}$ \\
\hline MAPE & 0.2120 & 0.2166 & 0.2169 & 0.2270 & 0.2238 & 0.2280 \\
Mean & -0.0219 & -0.0259 & -0.0305 & -0.0355 & -0.0416 & -0.0414 \\
Skew & -0.1664 & -0.1780 & -0.2542 & -0.1331 & -0.2381 & -0.2877 \\
Kurtosis & 0.5054 & 0.4790 & 0.1893 & 0.3756 & 0.2070 & 0.0412 \\
Lag-1 Auto-correlation & 0.8497 & 0.8844 & 0.8798 & 0.8829 & 0.8806 & 0.8911 \\
Lag-2 Auto-correlation & 0.6599 & 0.6961 & 0.6875 & 0.6969 & 0.6976 & 0.7137 \\
\hline
\end{tabular}

The histograms in Figure 3.1 demonstrate that the forecast errors do not conform to a normal 


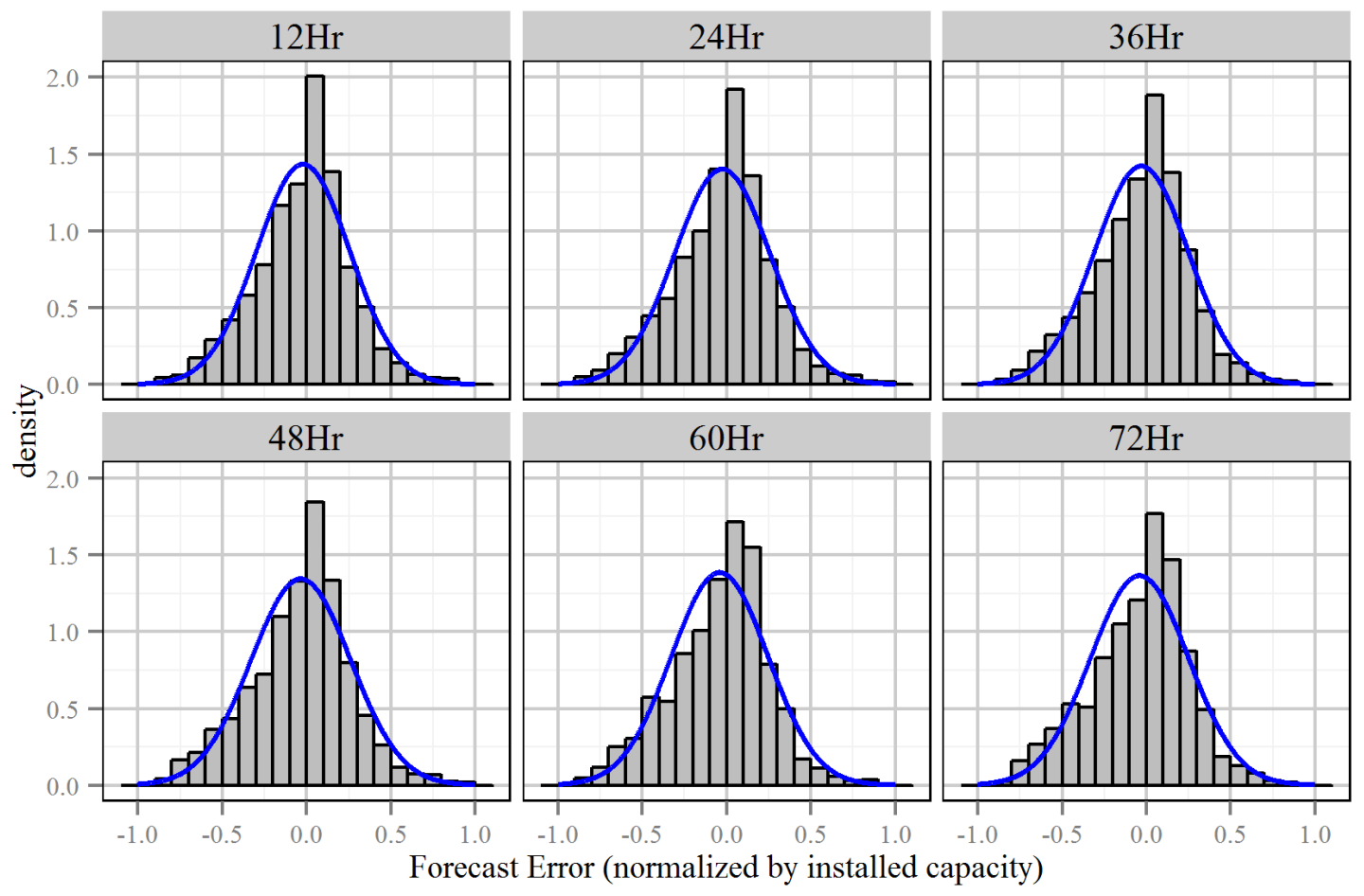

Figure 3.1: Histograms of observed wind generation forecast error (normalized by installed wind capacity) at a range of lead times overlaid with a normal distribution fitted to the data

distribution. Observation of the statistics in Table 3.1 reveals a positive kurtosis indicating that the distribution is more peaked than a normal distribution and has heavier tails. (Note that the kurtosis values displayed here are technically the excess kurtosis, the kurtosis in exceedence of the kurtosis of a normal distribution, which is always 3.) Additionally the forecast error has a negative skew as well as a negative mean, which becomes increasingly negative as the lead time increases. This indicates that the forecast methodology employed for this system has a slight bias to under predict the wind generation. The hourly forecast errors also exhibit a strong lag-1 and lag-2 auto-correlation.

While the hydropower scheduling methodology employed for our study optimizes resources based on net load forecasts for an entire week, generation is only scheduled for a 24 hour horizon, so the model is most sensitive to the characteristics of the day-ahead forecast. It is therefore on the 
day-ahead forecast that the attention is focused in developing the synthetic forecast methodology. Also it is necessary to point out that a day-ahead forecast would technically be a forecast for 24 hours with lead times ranging from 12 to 36 hours (assuming that generation is scheduled at noon for the following calendar day, which is typical for many day-ahead energy markets). Observation of the error distributions for the 12 hour, 24 hour and 36 hour forecasts, however, shows that there are only minor differences in the distributions across these lead times. Using the 24 hour forecast error characteristics as a basis for developing a day-ahead forecast error model is therefore considered a reasonable approximation.

\subsubsection{Day-ahead Wind Forecast Model}

Analysis of the observed forecast error data suggests that existing ARMA models could be improved upon to capture some of the non-Gaussian characteristics of the error distribution. The wind forecast model we employ applies a Box-Cox transformation to the error data before fitting an ARMA model to the transformed error. As described below, this allows a standard ARMA model with a random normal variable to be used to generate a forecast time series with errors that are not normally distributed.

First the forecast error is normalized by the installed capacity.

$$
\epsilon_{t, o b s}^{W}=\frac{P_{t, o b s}^{W F}-P_{t}^{W}}{P_{\max }^{W}}
$$

In equation 3.5, $\epsilon_{t, o b s}^{W}$ is the observed normalized wind forecast error, $P_{t, o b s}^{W F}$ is the observed 24 hour lead time wind generation forecast (MW) for time $t$, and $P_{t}^{W}$ is the actual wind generation (MW) at time t. $P_{m a x}^{W}$ is the installed wind capacity (MW). Then the Box-Cox transformation is applied to the normalized forecast error.

$$
\epsilon_{t, o b s}^{\prime}=\frac{\left(\epsilon_{t, o b s}^{W}+\lambda_{2}\right)^{\lambda_{1}}-1}{\lambda_{1}}
$$

$\epsilon_{t, o b s}^{\prime}$ in equation 3.6 is the transformed error, and $\lambda_{1}$ and $\lambda_{2}$ are parameters of the Box-Cox transformation. The mean is then removed resulting in a distribution that is approximately normal 
centered on a mean of zero.

$$
\epsilon_{t, o b s}^{*}=\epsilon_{t, o b s}^{\prime}-\mu^{\prime}
$$

$\mu^{\prime}$ is the mean of the transformed error, and $\epsilon_{t, o b s}^{*}$ is the mean-removed transformed error to which the ARMA model is fitted. The resulting distributions before and after the Box-Cox transformation are shown in Figure 3.2.
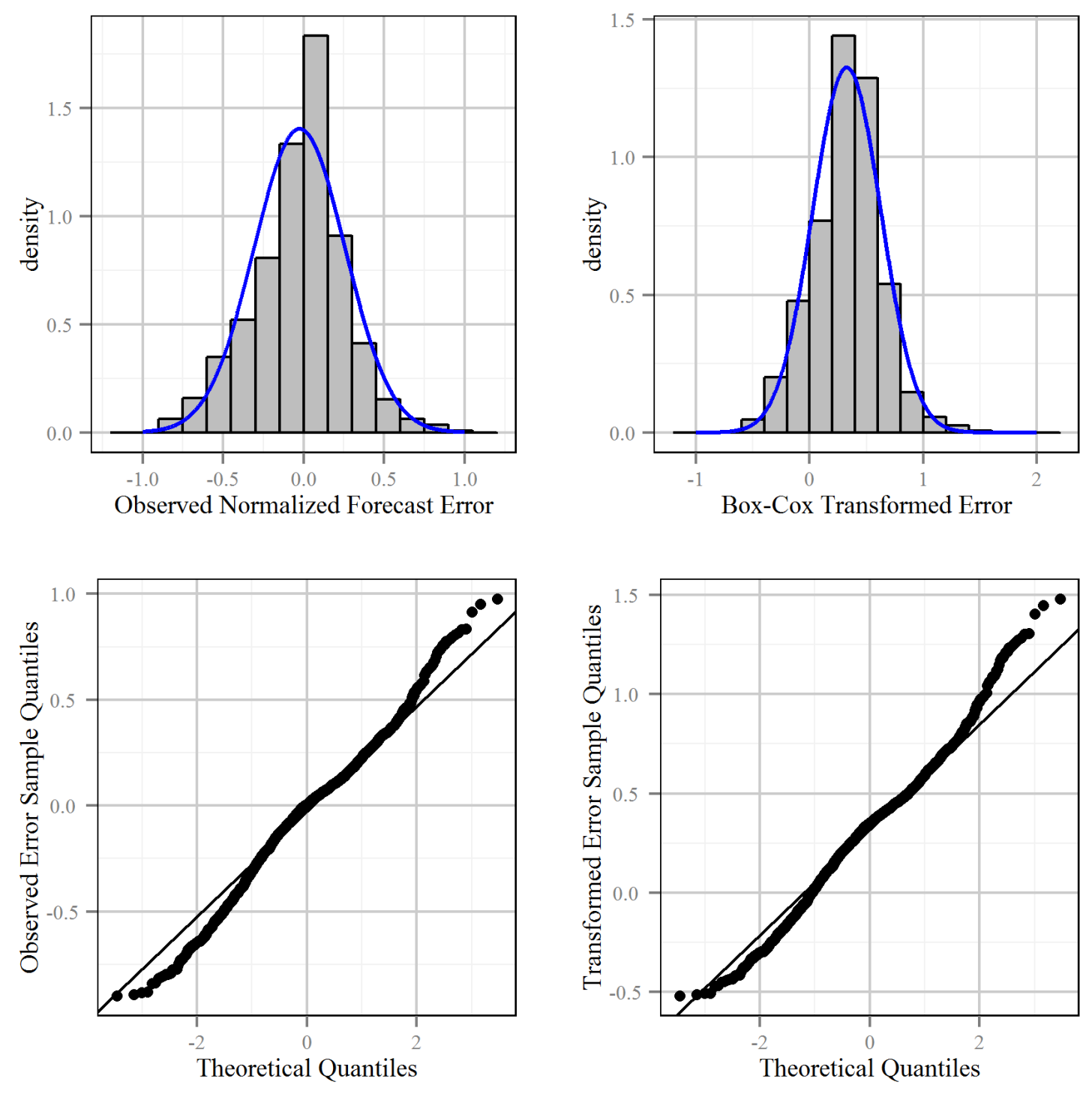

Figure 3.2: Histograms overlaid with normal distributions and QQ-Normal plots of observed wind forecast error and Box-Cox transformed error 
ARMA models up to order $(2,2)$ are then fit to the mean-removed transformed data, and the model with the lowest AIC score is selected as the best fit. A general ARMA model of order (p,q) is shown in equation 3.8 where the $\alpha$ and $\beta$ coefficients are parameters, and $N$ is a random normal variable with mean of 0 and standard deviation of $\sigma$. AIC scores from the model fitting and parameter values of the best fit $\operatorname{ARMA}(2,1)$ model and the Box-Cox transformation are shown in Tables 3.2 and 3.3.

$$
\epsilon_{t}=\alpha_{1} \epsilon_{t-1}+\alpha_{2} \epsilon_{t-2}+\ldots+\alpha_{p} \epsilon_{t-p}+\beta_{1} N_{t-1}(0, \sigma)+\ldots+\beta_{q} N_{t-q}(0, \sigma)+N_{t}(0, \sigma)
$$

Table 3.2: ARMA model fitting AIC values

\begin{tabular}{rr}
\hline Order ARMA(p,q) & AIC \\
\hline$(1,0)$ & -2029.53 \\
$(1,1)$ & -2303.87 \\
$(1,2)$ & -2316.41 \\
$(2,0)$ & -2345.96 \\
$(2,1)$ & -2357.30 \\
$(2,2)$ & -2299.87 \\
\hline
\end{tabular}

Table 3.3: Wind forecast error model Box-Cox and $\operatorname{ARMA}(2,1)$ parameter values

\begin{tabular}{rrrrrrrr}
\hline Parameter & $\lambda_{1}$ & $\lambda_{2}$ & $\alpha_{1}$ & $\alpha_{2}$ & $\beta_{1}$ & $\sigma^{\prime}$ & $\mu^{\prime}$ \\
\hline Value & 1.255 & 1.331 & 1.526 & -0.650 & -0.364 & 0.128 & 0.326 \\
\hline
\end{tabular}

The $\operatorname{ARMA}(2,1)$ model has the lowest AIC score and is therefore used to generate synthetic Box-Cox transformed forecast error.

$$
\begin{gathered}
\epsilon_{t, \text { model }}^{*}=\alpha_{1} \epsilon_{t-1, \text { model }}^{*}+\alpha_{2} \epsilon_{t-2, \text { model }}^{*}+\beta_{1} N_{t-1}\left(0, \sigma^{\prime}\right)+N_{t}\left(0, \sigma^{\prime}\right) \\
\epsilon_{t, \text { model }}^{\prime}=\epsilon_{t, \text { model }}^{*}+\mu^{\prime}
\end{gathered}
$$

The error term from the model is then transformed back and applied to the actual wind generation at the given time step to produce the initial wind forecast value, $P_{t, \text { model }}^{W F^{\prime}}$.

$$
\epsilon_{t, \text { model }}^{W}=\left(\epsilon_{t, \text { model }}^{\prime} \lambda_{1}+1\right)^{1 / \lambda_{1}}-\lambda_{2}
$$




$$
p_{t, \text { model }}^{W F^{\prime}}=P_{t}^{W}+\epsilon_{t, \text { model }}^{W} P_{\max }^{W}
$$

The resulting forecast value is checked for feasibility. If the initial forecast value is negative or greater than capacity, the forecast value, $P_{t, \text { model }}^{W F}$, is set to zero or maximum capacity respectively. The values of $\epsilon_{t, \text { model }}^{*}$ and $N_{t}\left(0, \sigma^{\prime}\right)$ are then adjusted to correspond to this forecast value before applying the ARMA model for the next time step.

$$
p_{t, \text { model }}^{W F}=\left\{\begin{array}{lc}
0, & p_{t, \text { model }}^{W F^{\prime}}<0 \\
p_{t, \text { model }}^{W F^{\prime}}, & 0 \leq p_{t, \text { model }}^{W F^{\prime}} \leq P_{\max }^{W} \\
P_{\text {max }}^{W}, & p_{t, \text { model }}^{W F^{\prime}}>P_{\text {max }}^{W}
\end{array}\right.
$$

Figure 3.3 displays the histograms of the observed 24 hour forecast error and 100 traces of forecast errors generated by applying the forecast model to the same actual wind generation time series. The corresponding statistics are presented in Table 3.4.

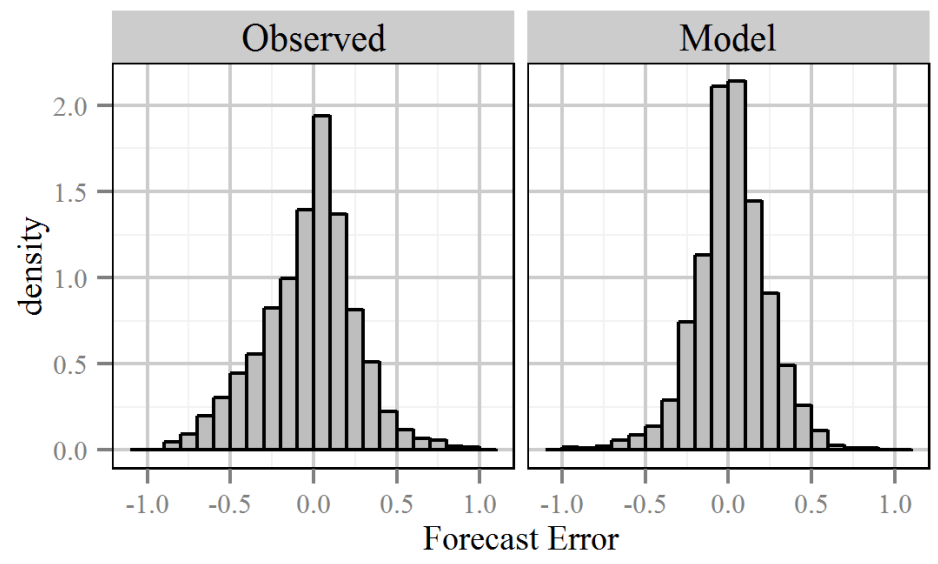

Figure 3.3: Histograms of observed wind generation forecast error and 100 traces of modeled forecast error normalized by installed wind capacity

The results in Table 3.4 indicate that the forecast error model produces less variance in the error outputs than the observed forecast error. It also has a resulting mean that is slightly positive whereas the observed forecast error has a slightly negative mean, and the model skew is not as negative as the observed skew. The model does a better job of reproducing the excess kurtosis than 
Table 3.4: Statistics of observed and model forecast percent error distributions (Model values are mean values from 100 traces.)

\begin{tabular}{rrr}
\hline & Observed & Model \\
\hline MAPE & 0.216 & 0.164 \\
Mean & -0.025 & 0.042 \\
Standard Deviation & 0.284 & 0.213 \\
Skew & -0.185 & -0.010 \\
Kurtosis & 0.487 & 0.639 \\
Lag-1 Auto-correlation & 0.884 & 0.847 \\
Lag-2 Auto-correlation & 0.696 & 0.630 \\
\hline
\end{tabular}

would occur if a normal distribution were assumed, and it does a relatively good job of capturing the lag-1 and lag-2 auto-correlation. Additional analysis suggests that the differences in mean, variance and skew come from the fact that the forecast model does not capture the dependency of forecast error on the actual generation. In cases where the actual wind generation is low, an actual wind forecast will tend to over-predict rather than under-predict the generation. The opposite is the case when actual generation is high. The model does not tend to capture the magnitude of the negative forecast errors very well in the case of high actual wind generation. Further analysis on this is presented in Appendix B.

The wind generation forecast model (equations 3.9 to 3.13 ) is then applied to the actual wind generation time series for the $10 \%$ penetration case for each scenario to be modeled using the parameter values from Table 3.3. The resulting percent error from each trace is then used to calculate the forecast value for each of the remaining penetration levels. The $10 \%$ penetration case has the greatest relative variability, so using this actual wind generation series to generate the forecast error time series assures that infeasible values do not result when scaling the forecast from one penetration level to another. This allows the forecast percent error to remain constant across all penetration levels for a given forecast trace within a given scenario. A time series plot of the actual wind and a single trace of the day-ahead forecast for the spring scenario $30 \%$ penetration case is shown in Figure 3.4. 


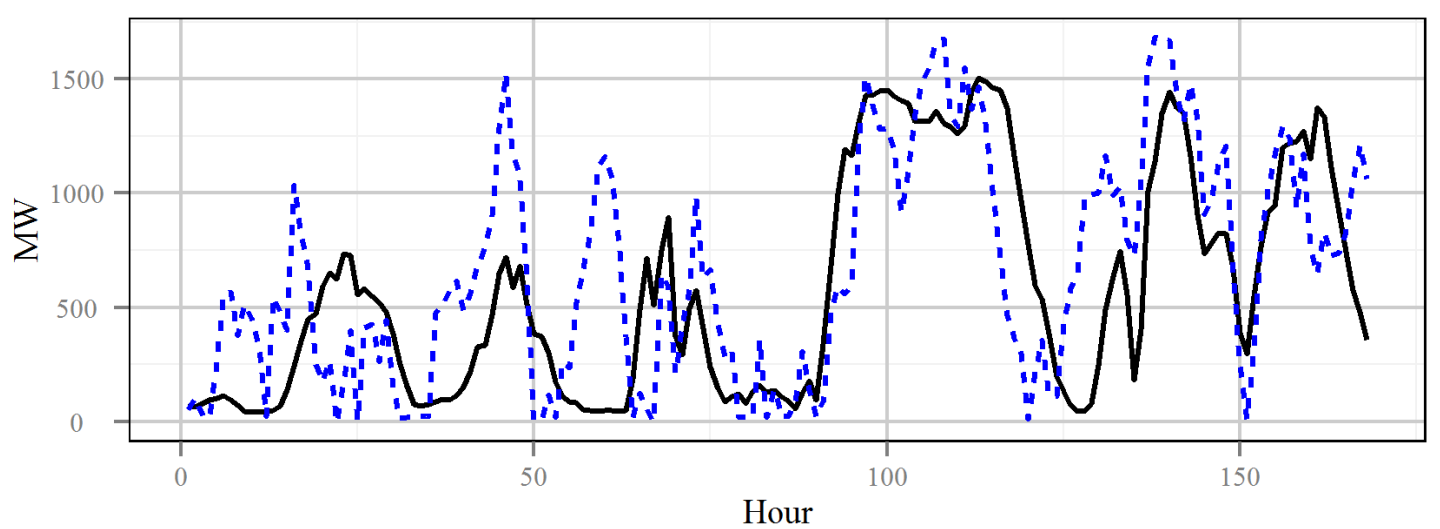

- Actual Wind Generation - - - Forecast Wind Generation

Figure 3.4: Sample trace of model forecast wind generation and actual generation, spring scenario, $30 \%$ penetration

\subsubsection{Wind Forecast Models for Two and Three Days Ahead}

For the two day ahead forecast error, $\epsilon_{t, \text { model }}^{W 2}$, an additional error term, $\epsilon_{t, \text { model }}^{\Delta 2}$ is added to the day-ahead forecast error at that time step.

$$
\epsilon_{t, \text { model }}^{W 2}=\epsilon_{t, \text { model }}^{W}+\epsilon_{t, \text { model }}^{\Delta 2}
$$

The additional error term is generated by an ARMA model based on the differences between observed 48 hour and 24 hour forecasts, $\epsilon_{t, o b s}^{\Delta 2}$.

$$
\epsilon_{t, o b s}^{\Delta 2}=\epsilon_{t, o b s}^{W 48}-\epsilon_{t, o b s}^{W 24}
$$

In this case the additional error is assumed to be normally distributed. Once again an ARMA $(2,1)$ model has the lowest AIC score and is selected as the best model. The model parameters are shown in Table 3.5.

The three day ahead forecast error model is similar. An additional error term is added to the two day error.

$$
\epsilon_{t, \text { model }}^{W 3}=\epsilon_{t, \text { model }}^{W 2}+\epsilon_{t, \text { model }}^{\Delta 3}
$$


where the observed differences between the 72 and 48 hour forecast error are used to fit the model. In this case an $\mathrm{AR}(2)$ model has the lowest AIC score and is used to generate the additional three day forecast error. Histograms of observed forecast error for lead times of 48 and 72 hours along with results from 100 traces of model forecast error for the same lead times (representing two day ahead and three day ahead forecasts) are shown in Figure 3.5 with the corresponding statistics in Table 3.6.

Table 3.5: Parameter values for additional error models for two day ahead and three day ahead wind generation forecasts

\begin{tabular}{rrrrrr}
\hline & $\alpha_{1}$ & $\alpha_{2}$ & $\beta_{1}$ & $\sigma$ & $\mu$ \\
\hline Two-day Forecast & 0.329 & 0.270 & 0.543 & 0.066 & -0.0078 \\
Three-day Forecast & 0.930 & -0.132 & NA & 0.068 & -0.0067 \\
\hline
\end{tabular}

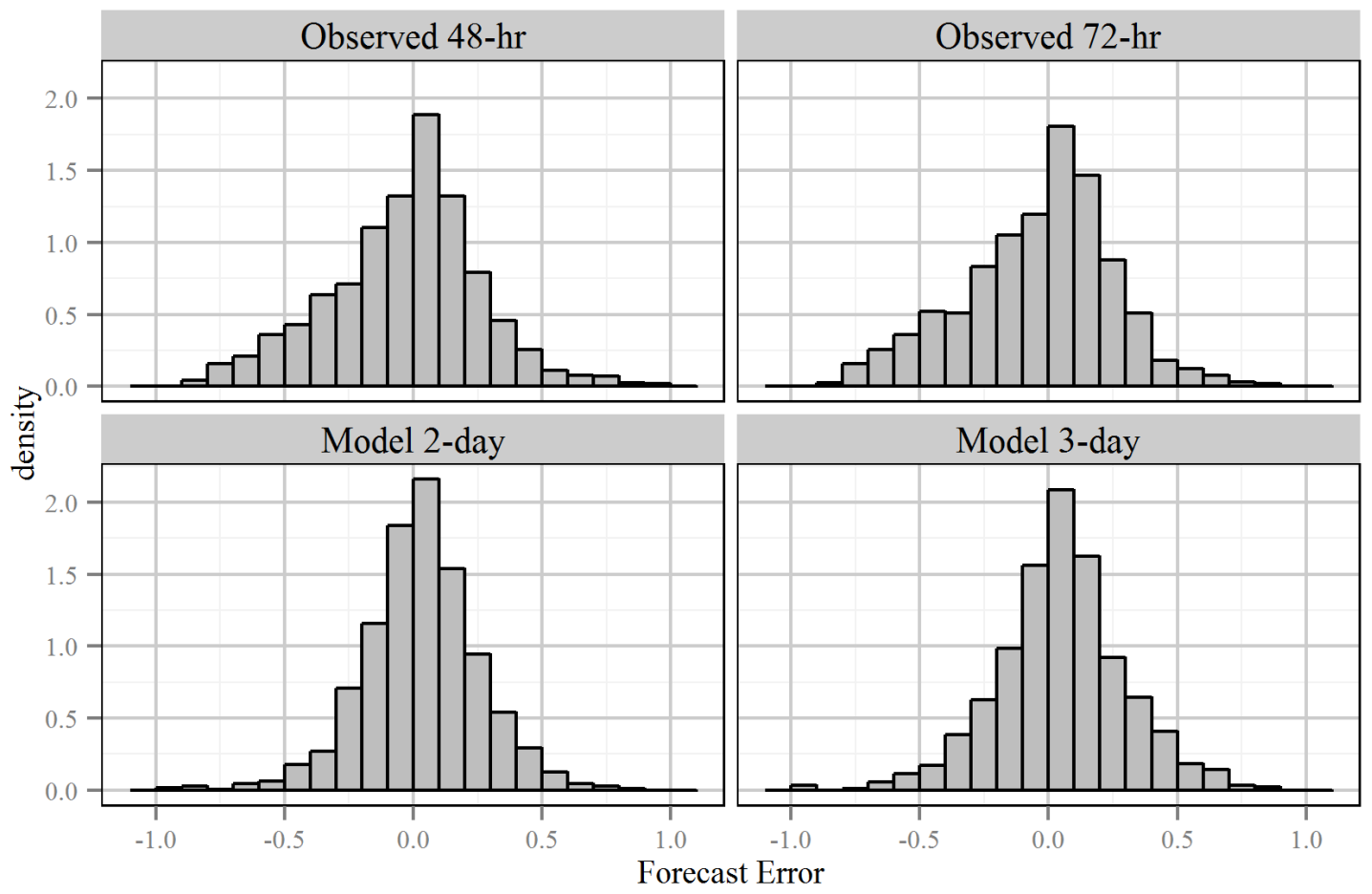

Figure 3.5: Histograms of observed and model 48 hour and 72 hour wind generation forecast error normalized by installed capacity 
Table 3.6: Statistics of observed and model 48 hour and 72 hour wind generation forecast error normalized by installed capacity

\begin{tabular}{r|rr|rr}
\hline & $\begin{array}{r}\text { 48-hour } \\
\text { Observed }\end{array}$ & $\begin{array}{r}\text { 2-day } \\
\text { Model }\end{array}$ & $\begin{array}{r}\text { 72-hour } \\
\text { Observed }\end{array}$ & $\begin{array}{r}\text { 3-day } \\
\text { Model }\end{array}$ \\
\hline MAPE & 0.227 & 0.176 & 0.228 & 0.193 \\
Mean & -0.035 & 0.052 & -0.041 & 0.064 \\
Standard Deviation & 0.297 & 0.226 & 0.292 & 0.243 \\
Skew & -0.133 & -0.040 & -0.288 & -0.056 \\
Kurtosis & 0.376 & 0.685 & 0.041 & 0.563 \\
Lag-1 Auto-Correlation & 0.883 & 0.828 & 0.891 & 0.820 \\
Lag-2 Auto-Correlation & 0.697 & 0.608 & 0.714 & 0.605 \\
\hline
\end{tabular}

The two day ahead model is applied to the day ahead forecast traces used as inputs for the integrated hydropower and wind model to generate the corresponding two day ahead forecast for each trace. The same is done with the three day forecast. The three day ahead forecast for a given trace is used for all lead times of three days or more for that trace. 


\section{Chapter 4}

\section{Assessing the Value of Integrated Hydropower and Wind Generation}

\subsection{Introduction}

\subsubsection{Overview}

The rapid growth in installed wind generation capacity in recent years puts greater demand on power systems to maintain sufficient flexible generating resources and balancing reserves. Hydropower has often been considered one of the best balancing resources to couple with wind generation. Hydro generators have high ramp rates and often have automatic generation control (AGC) capabilities enabling quick response to imbalances in energy supply and demand. Hydropower generation has a low marginal cost, and the fuel supply, water, is a renewable resource. Most conventional hydropower facilities also have the capability to store energy in the form of impounded water allowing for a level of control over the timing of generation, making use of the energy when most needed. ${ }^{1}$ Hydropower, therefore, plays an increasingly valuable role as part of a sustainable energy portfolio by not only producing renewable energy itself, but also by making it possible to incorporate larger amounts of other renewable generation, such as wind, through the flexible balancing resources (ancillary services) it provides.

Unlike other power generating resources, however, hydropower facilities are generally designed and operated to serve multiple objectives. These objectives may include flood control, environmen-

\footnotetext{
${ }^{1}$ Pumped storage hydropower facilities (PSH) provide the added benefit of using excess energy for pumping water to an upper reservoir to be used for generation in times of higher energy demand thus providing additional balancing reserves. Only conventional hydropower is considered in our study, though the methodology could be extended to systems with PSH.
} 
tal objectives including habitat protection and fisheries management, water storage and delivery, navigation, water quality and recreation in addition to power generation. The specific objectives and their relative priorities are unique to each system, but in general water objectives have a higher priority in system operating policy than the power objectives. That is to say that the water management objectives cannot be compromised for power generation purposes. These non-power policies restrict the allowable outflows from hydropower facilities effectively reducing system flexibility. It is necessary to account for this reduced flexibility from non-power policy when evaluating the capacity of the hydropower system for balancing integrated wind generation.

\subsubsection{Existing Wind and Hydropower Integration Studies}

In 2011, the International Energy Agency (IEA) Task 24 Integration of Wind and Hydropower Systems Final Technical Report was released (Acker, 2011a,b). This report brings together case studies from participants in seven countries to investigate wind integration in electric systems with large amounts of hydropower generation. It analyzes benefits, challenges and impacts of wind integration in systems with varied characteristics in their market structures, hydropower systems and electrical systems. A summary of the case studies and their findings is presented in Acker et al. (2012b) and shows that in many cases existing hydropower systems can provide the balancing reserves necessary for integrating large amounts of wind generation. Some case studies expect significant operational challenges for high levels of wind penetration (greater than 20\%). A review of existing literature on wind and hydropower integration, including studies which focus on developing scheduling algorithms for coordinated hydropower and wind generation is provided in Chapter 1.

One other large-scale study of note in regards to wind and hydropower integration is the Western Wind and Solar Integration Study which evaluates the feasibility and impacts of up to $30 \%$ wind and $5 \%$ solar in the WestConnect footprint (GE Energy, 2010). The WWSIS shows a significant increase in operating costs for the entire system if the flexibility of hydropower resources for balancing the variability of renewable generation is removed. One common conclusion from 
both the IEA Task 24 Report and the hydropower analysis from the WWSIS is that there is a need for improved hydropower modeling in wind integration studies. In a companion study that carries out a detailed analysis of the hydropower modeling in the WWSIS, Acker and Pete (2012) find that modeling assumptions at times lead to an overestimation of true hydropower capacity, particularly in low flow years. In another companion report to the WWSIS, Hodge et al. (2011) note the importance of modeling non-power constraints when trying to accurately capture the impacts of wind generation on hydropower systems. The IEA Task 24 Report calls for improvements in the modeling of both physical processes, such as mass balances, and constraints on the system (Acker et al., 2012b).

\subsubsection{Significance of this Research}

The research we present here contributes to improved hydropower modeling for wind integration studies by developing a methodology that not only models the physical processes of the hydropower system but also accounts for the limitations resulting from both power-related and non-power policy constraints. The methodology utilizes the optimization solver in RiverWare, a hydropower modeling tool, to model hydropower operations with a set of prioritized policy constraints and objectives based on realistic policies that govern the operation of actual hydropower systems. This captures the effective capacity and flexibility of a hydropower system that must be considered by system operators when coordinating hydropower resources with wind generation.

In addition to realistic modeling of hydropower operations and policy, the methodology also produces an economic evaluation of integrated wind and hydropower that not only accounts for the value of energy from the integrated system but also for the value of ancillary services provided by the hydropower resources. With the relatively recent development of markets for ancillary services in many regions, ancillary services can carry an explicit economic value in addition to their value in maintaining grid stability. Ancillary services can provide a significant portion of hydropower revenue and should therefore be accounted for when determining the total value of the system.

The end value of integrated hydropower and wind generation is specific to each power system 
and is dependent on numerous factors. In consideration of this, the goal here is not to establish a specific value for integrated wind generation. The point is rather to demonstrate the importance of modeling realistic hydropower operations, including non-power constraints, when considering the value of integrated hydropower and wind generation. In addition the sensitivity of the value of wind integration to various conditions and system parameters is evaluated. To do this, the proposed methodology is applied to a sample hydropower system with integrated wind generation under a range of scenario conditions. By comparing effects on economic outputs, operational conditions and constraint satisfaction from modifying various system parameters, conclusions are drawn about key modeling assumptions and system characteristics and their effect on the evaluation of hydropower and wind integration.

\subsection{Modeling and Methodology}

\subsubsection{Conceptual Model}

The manner in which a hydropower system is scheduled and operated is dependent to a significant degree on the organizational structure of the larger system of which it is a part and the market structures which exist in the system's balancing authority area (BA). The organizational model we propose here is a hybrid of a traditional, vertically integrated utility and a formal energy market. The hydropower system is a sub-system within a BA, such as a large-scale utility which owns multiple hydropower resources. These resources must be dispatched to meet local load and reserve requirements. Additional capacity can be utilized to bid into formal markets for energy and ancillary services. Such a mixed model is representative of organizational structures in the Western

Electricity Coordinating Council (WECC) area where power producers must meet load obligations in their own BA but also have the opportunity to participate in the California Independent System Operator (CAISO) market (Loose, 2011). Energy markets improve conditions for integrating large amounts of wind generation by providing sub-hourly energy markets that redispatch every five to fifteen minutes (Milligan et al., 2009). In our model, participation in the market occurs in only 
one direction. Energy and ancillary services can be sold into the market when the hydro system has excess capacity, but they cannot be purchased from the market to help meet local requirements when capacity is limited. All resources to meet local requirements must come from within the system being modeled. All modeling is at an hourly time step. All sub-hourly processes are therefore approximated by hourly averages.

The utility is assumed to have must-take contracts with a set of geographically dispersed wind farms. Wind is incorporated into the model as negative load. One important note is that no explicit economic value is assigned to the energy and reserves used to meet the local load and reserve requirements. Meeting load and reserve requirements is treated as a constraint for the system. Only the energy and ancillary services sold into the market are given an explicit economic value. Also wind is not assigned a purchase cost. Rather the output returns the net value of added wind generation by comparing the total value from a Base case without added wind generation to the same scenario with wind generation. This is in contrast to many previous studies which have used flat blocks of equivalent wind energy without the variability as the base case to establish the cost of wind integration. Milligan and Kirby (2009) address the issues with using the flat wind block approach.

\subsubsection{Hydropower Modeling in RiverWare}

\subsubsection{RiverWare Optimization}

Hydropower modeling for our study is carried out using the optimization solution in RiverWare, a general tool for hydropower, river and reservoir system modeling developed at the Center for Advanced Decision Support for Water and Environmental Systems (CADSWES) at the University of Colorado. RiverWare's optimization solution is a pre-emptive linear goal program in which the user creates prioritized goals, which are constraints and objectives that represent the operating policy of the system. Then a linear program is solved at each priority level. When the goal is written as a soft constraint (or multiple soft constraints), the objective function of the linear 
program at that priority is to minimize the deviations from the constraint values. In the case that not all soft constraints at a given priority level can be satisfied the optimization minimizes the maximum deviation then re-solves the linear program until all constraints at that priority are satisfied (known as a repeated minimax solution). Once a soft constraint has been satisfied at a higher priority, it will not be violated to meet a lower priority soft constraint or objective. This approach accounts for the fact that not all policy constraints are of equal importance. For example target environmental flow constraints may not be satisfied if it would require violating license minimum or maximum storages (pool elevations), but environmental flow constraints will be satisfied before optimizing power generation. Additional information about optimization in RiverWare, including the formulation of automatic physical constraints, is provided by Eschenbach et al. (2001).

\subsubsection{Power Modeling}

Hydropower generation is modeled at the plant level as a function of operating head and turbine release. Each plant's power characteristics are represented by a set of piecewise linear curves, one for each operating head, with linear interpolation between curves to calculate power at intermediate operating heads. In the optimization solution, operating head is held constant at a user-input estimated value. A post-optimization simulation run uses portions of the optimization solution to set releases. It then uses an iterative solution to calculate the operating head and power generation. This removes the approximation error introduced by assuming a constant operating head in the optimization solution.

\subsubsection{Ancillary Services}

The classification of ancillary services or operating reserves varies in different regions (Ela et al., 2011). Four marketable ancillary services are defined in our model, consistent with the ancillary services products available in the CAISO market: regulation up, regulation down, spinning reserve and non-spinning reserve. Load following reserve is a fifth ancillary service that is modeled only as a local resource and represents all other reserve capacity scheduled to balance any variability 
or forecast error in the net load beyond the regulation time scale. Load following reserve is modeled as a bi-directional reserve and carries the same incremental and decremental capacity. In general, the incremental reserves at a hydropower plant are constrained by

$$
R U_{r, t}+S R_{r, t}+N S_{r, t}+L F_{r, t} \leq H C_{r, t}-P_{r, t}
$$

where $R U_{r, t}, S R_{r, t}, N S_{r, t}$ and $L F_{r, t}$ represent regulation up capacity, spinning reserve, non-spinning reserve and load following reserve respectively, $H C_{r, t}$ represents maximum hydropower capacity at time $t$ and $P_{r, t}$ represents hydropower generation at time $t . H C_{r, t}$ is a function of the operating head and the maximum turbine release at that operating head. Decremental reserves are constrained by

$$
R D_{r, t}+L F_{r, t} \leq P_{r, t}-P_{r, t}^{\min }
$$

where $R D_{r, t}$ is regulation down capacity, and $P_{r, t}^{m}$ in is the minimum allowable generation at the plant. Additional constraints in the policy set further restrict the hydro capacity at certain hydropower plants based on flow restrictions from non-power constraints. This captures the manner in which non-power policy effectively limits the flexibility of certain hydropower plants in providing ancillary services.

If reserve capacity is actually deployed to generate energy (or to reduce generation in the case of regulation down), then this quantity of energy is removed from the reserve capacity to avoid double counting the reserves. The deployment of ancillary services at time scales shorter than one hour, such as regulation, cannot be modeled directly. Instead a deployment fraction is used to represent the fraction of the reserve that is deployed in actual generation on average over the time step. For further details on the ancillary services modeling, refer to Section 2.3.3.

\subsubsection{Economic Modeling}

The value of energy sold at a given time step is based on a piecewise linear function. For each additional block of energy, the unit value decreases. This represents the effect that bidding additional energy that has a low marginal cost (e.g. wind energy or hydropower) has on reducing the 
market clearing price. Because the day-ahead energy bid comes from the scheduling run using dayahead wind forecasts (as described in 2.2), this also approximates the effect high wind generation forecasts can have on reducing energy prices (see Jónsson et al., 2010). The economic value of each ancillary service (regulation up, regulation down, spinning reserve and non-spinning reserve) is modeled similarly to energy using a piecewise linear function; however the value of ancillary services also includes the compensation for the energy generated when the reserve capacity is deployed. The deployment value is based on the real-time market clearing price for energy.

\subsubsection{Other Physical Processes}

RiverWare provides the capability to model numerous physical processes in the hydropower system. Among those included in our model are reservoir mass balances and pool elevations, routing between reservoirs and tailwater elevation. Refer to Zagona et al. (2001) and the RiverWare Documentation (CADSWES, 2012) for details on how these processes are modeled within RiverWare. Section 2.4 contains details on the specific methods used in our model for this study and the parameter values within the model.

\subsubsection{Run Sequence}

The model run sequence consists of a two-stage optimization which is intended to represent actual operations where generating resources are scheduled based on net load forecasts then must be adjusted to meet real-time conditions. First a one week scheduling run is carried out using wind generation forecasts and load forecasts as inputs. This is followed by a 24 hour operations run which uses actual wind generation and load inputs. The objective of the scheduling run is to maximize the combined value of energy and ancillary services sold in the day-ahead market for the entire week after meeting all constraints, including target ending elevations for each reservoir, as well as local load and reserve requirements. The purpose of the one-week scheduling run is for the system to look far enough into the future to position itself to be able to meet anticipated conditions when scheduling for the current day. 
A set of outputs from the first 24 hours of the scheduling run are passed to the operations run. These include energy and ancillary services committed into the day-ahead market, generation, reserves and releases scheduled at each hydropower plant and scheduled pool elevations at each reservoir at the end of each six hour period for the next 24 hours. The objective of the operations run is to maximize the value of energy in storage. The operations run is divided into four individual six hour runs. This division into six hour runs balances a representation of reasonable flexibility of the hydro resources to adjust generation based on updated forecasts without an overestimation of system flexibility from modeling perfect forecast knowledge over the entire 24 hour run period. Alternative scenarios include a real-time market in the operations run with an objective to maximize the value of energy sold in the real-time market. After the operations run, the model advances 24 hours with the ending reservoir storages becoming the new initial conditions. Updated load and wind forecasts are imported before starting a new one-week scheduling run. The process is repeated to produce one full week of operations run outputs. The model run sequence is described in greater detail in Section 2.2.

\subsection{Wind Modeling}

\subsubsection{Actual Wind}

Wind generation data are based on Bonneville Power Administration (BPA) Balancing Authority 2010 total wind generation data. ${ }^{2}$ BPA five minute data are averaged to hourly values for use in our study and then scaled to represent the desired wind penetration levels: $10 \%, 20 \%, 30 \%$, and $40 \%$. The $40 \%$ wind penetration case is recognized as a relatively extreme penetration scenario and is included here only to evaluate system behavior at the upper limits of wind integration. Wind variability does not remain constant as the level of penetration changes. The wider geographical distribution of wind farms typically associated with increased penetration results in less correlation between generators and thus reduced relative variability (Wan, 2004, 2005). In order to account for this, the variability in the wind data is scaled as well as the mean. The total BPA wind capacity

\footnotetext{
${ }^{2}$ http://transmission.bpa.gov/Business/Operations/Wind/default.aspx
} 
from 2010 is approximately equal the capacity for the $40 \%$ penetration case in our model. The relative variability is therefore increased from the scaled observed data for the lower penetration scenarios. The BPA wind generation data are only available in aggregate, so wind data from select sites in the WWSIS 2006 dataset $^{3}$ are used to estimate a reasonable change in variability. WWSIS sites are selected such that the total annual mean generation and the annual standard deviation of wind generation at ten minute intervals are approximately equal to the BPA 2010 mean and ten minute standard deviation. Then sites are removed from the selected WWSIS sites so that the annual mean generation is equal to that of the $30 \%, 20 \%$, and $10 \%$ wind cases. With each reduction the geographic spread of the wind sites is decreased which has the effect of increasing the relative variability. The four cases based on the WWSIS data are then converted to hourly wind data, and the annual 60-minute standard deviation is then calculated for each as well as the 60 -minute standard deviation for the BPA wind data. The ratio of the synthetic 60-minute standard deviation to the BPA 60-minute standard deviation is then used to scale the deviation of wind generation from the scaled mean at each time step.

$$
\begin{gathered}
P_{p e n, t}^{W}=\bar{P}_{p e n}^{W}+\frac{\sigma_{60}^{p e n}}{\sigma_{60}^{B P A}}\left(P_{B P A, t}^{W}-\bar{P}_{B P A}^{W}\right) \\
\bar{P}_{p e n}^{W}=P_{p e n, \max }^{W} F_{C}
\end{gathered}
$$

$P_{p e n, t}^{W}$ is the "actual" wind generation value at time $t$ for the given penetration level pen. $\sigma_{60}^{p e n}$ is the 60-minute standard deviation for penetration level pen based on the WWSIS wind data, and $\sigma_{60}^{B P A}$ is the BPA 60 -minute standard deviation. $P_{B P A, t}^{W}$ is the observed BPA wind generation at time t. $P_{p e n, \max }^{W}$ is the installed wind capacity for penetration pen, and $F_{C}$ is the wind generation capacity factor which is assumed to be 0.3 for all penetration levels.

\footnotetext{
3 The WWSIS dataset is provided by the National Renewable Energy Laboratory, which is operated by the Alliance for Sustainable Energy, LLC for the U.S. Department Of Energy and is available at http://www.nrel.gov/wind/integrationdatasets/western/data.html
} 


\subsubsection{Wind Forecast}

In the absence of real forecast data corresponding to the BPA actual wind generation, synthetic wind generation forecasts are produced based a statistical analysis of combined observed actual wind generation data and wind generation forecast data from a set of sample wind farms. The goal with the synthetic forecast model we use here is not to reproduce forecasts that match those corresponding to the actual BPA wind generation data. Rather the purpose is to produce synthetic wind forecast time series that provide a reasonable and realistic characterization of wind generation forecast error recognizing that the specific characteristics of forecast error may be different for each system.

Previous wind integration studies have used autoregressive moving average (ARMA) models to produce synthetic wind forecasts. Matevosyan et al. (2009) employ an ARMA(1,1) model developed in Soder (2004) to develop a scenario tree of forecast errors for a stochastic optimization algorithm. The ARMA model parameters are fit to minimize the deviation in RMSE from observed forecast error over a range of forecast lead times. de Mello et al. (2011) develop a similar $\operatorname{ARMA}(1,1)$ model for wind generation forecast errors. The fitting of this model, however, also accounts for the cross-correlation between forecast errors at various time frames. The model is fit to minimize the weighted difference in observed and model values for mean, standard deviation, auto-correlation and cross-correlation with the forecast error series of shorter time frame.

ARMA models assume that the forecast error is normally distributed. Hodge and Milligan (2011) show that forecast errors in shorter time frames (five minutes to three hours) are not Gaussian but rather have higher kurtosis than a normal distribution. In general, a normal distribution would underestimate the number of time periods with very small and very large forecast error, and it would over predict the number of time periods with moderate forecast error. Analysis of the forecast error from the sample wind farms similarly reveals a kurtosis greater than that of normally distributed error as well as a negative skew (see Figure 4.1). In order to better capture these characteristics in our model, a Box-Cox transformation is first applied to the forecast error data, and then an 
ARMA model is fit to the mean-removed transformed data. An $\operatorname{ARMA}(2,1)$ model has the lowest AIC score and is thus used for the synthetic forecast model.

The observed normalized wind generation forecast error, $\epsilon_{t, o b s}^{W}$ is defined as

$$
\epsilon_{t, o b s}^{W}=\frac{P_{t, o b s}^{W F}-P_{t}^{W}}{P_{\max }^{W}}
$$

where $P_{t, o b s}^{W F}$ is the observed wind power forecast at time $t, P_{t}^{W}$ is the actual wind power, and $P_{\max }^{W}$ is the maximum wind power (installed capacity). A Box-Cox transformation is then applied to the normalized error with $\lambda_{1}$ and $\lambda_{2}$ as parameters, and the mean of the transformed error, $\mu^{\prime}$, is removed.

$$
\begin{gathered}
\epsilon_{t, o b s}^{\prime}=\frac{\left(\epsilon_{t, o b s}^{W}+\lambda_{2}\right)^{\lambda_{1}}-1}{\lambda_{1}} \\
\epsilon_{t, o b s}^{*}=\epsilon_{t, o b s}^{\prime}-\mu^{\prime}
\end{gathered}
$$

The $\operatorname{ARMA}(2,1)$ model with fitted parameters $\alpha_{1}, \alpha_{2}, \beta_{1}$ and $\sigma^{\prime}$ is then used to generate traces of the Box-Cox transformed forecast error.

$$
\begin{gathered}
\epsilon_{t, \text { model }}^{*}=\alpha_{1} \epsilon_{t-1, \text { model }}^{*}+\alpha_{2} \epsilon_{t-2, \text { model }}^{*}+\beta_{1} N_{t-1}\left(0, \sigma^{\prime}\right)+N_{t}\left(0, \sigma^{\prime}\right) \\
\epsilon_{t, \text { model }}^{\prime}=\epsilon_{t, \text { model }}^{*}+\mu^{\prime}
\end{gathered}
$$

The error term from the model is then transformed back and applied to the actual wind generation at the given time step to produce the wind forecast.

$$
\begin{gathered}
\epsilon_{t, \text { model }}^{W}=\left(\epsilon_{t, \text { model }}^{\prime} \lambda_{1}+1\right)^{1 / \lambda_{1}}-\lambda_{2} \\
p_{t, \text { model }}^{W F}=P_{t}^{W}+\epsilon_{t, \text { model }}^{W} P_{\text {max }}^{W}
\end{gathered}
$$

The resulting forecast value is checked for feasibility. If the forecast is negative or greater than capacity, the forecast value is set to zero or maximum capacity respectively. The values of $\epsilon_{t, \text { model }}^{*}$ and $N_{t}\left(0, \sigma^{\prime}\right)$ are then adjusted to correspond to this forecast value before applying the ARMA model for the next time step. The fitted model parameters are shown in Table 4.1. Histograms comparing 100 traces of the model forecast error distribution to the observed forecast error distribution are shown in Figure 4.1 with the corresponding statistics in Table 4.2. 
Table 4.1: Wind forecast error model Box-Cox and $\operatorname{ARMA}(2,1)$ parameter values

\begin{tabular}{rrrrrrrr}
\hline Parameter & $\lambda_{1}$ & $\lambda_{2}$ & $\alpha_{1}$ & $\alpha_{2}$ & $\beta_{1}$ & $\sigma^{\prime}$ & $\mu^{\prime}$ \\
\hline Value & 1.255 & 1.331 & 1.526 & -0.650 & -0.364 & 0.128 & 0.326 \\
\hline
\end{tabular}

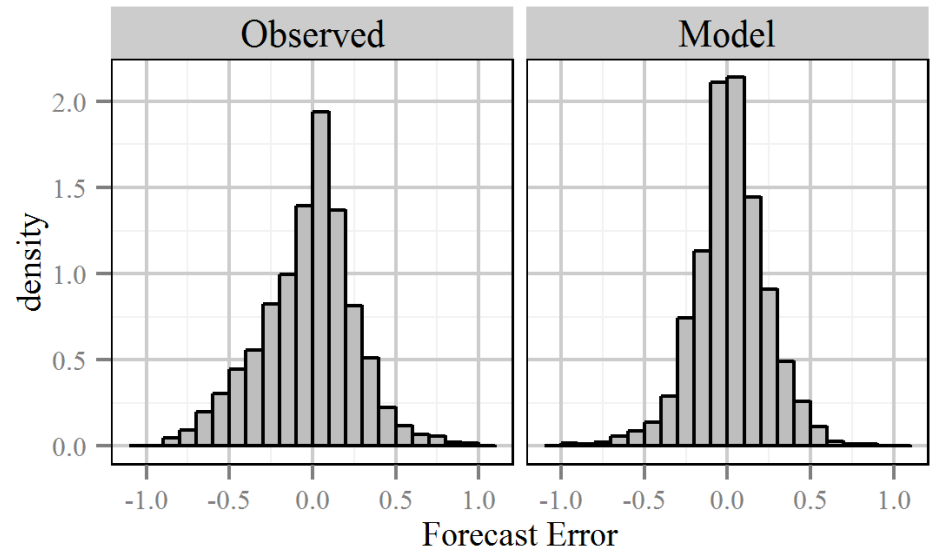

Figure 4.1: Histograms of observed wind generation forecast error and 100 traces of modeled forecast error normalized by installed wind capacity

Table 4.2: Statistics of observed and model forecast error distributions normalized by installed capacity (Model values are mean values from 100 traces.)

\begin{tabular}{rrr}
\hline & Observed & Model \\
\hline MAPE & 0.216 & 0.164 \\
Mean & -0.025 & 0.042 \\
Standard Deviation & 0.284 & 0.213 \\
Skew & -0.185 & -0.010 \\
Kurtosis & 0.487 & 0.639 \\
Lag-1 Auto-correlation & 0.884 & 0.847 \\
Lag-2 Auto-correlation & 0.696 & 0.630 \\
\hline
\end{tabular}

The $\operatorname{ARMA}(2,1)$ model is then applied to the wind generation time series used in our integrated wind and hydropower model scenarios. If it is assumed that power generation is scheduled at noon for the following calendar day, then a true day-ahead forecast would actually span lead times of 12 to 36 hours. Analysis of the observed forecast error data, however, shows little change in mean absolute percent error in forecasts with lead times ranging from 12 to 72 hours. Thus using a 
24 hour lead time forecast is considered here as a reasonable approximation of a day-ahead forecast. For forecasts of lead times greater than one day, an $\operatorname{ARMA}(2,1)$ model is applied to generate the additional error added to the 24 hour error. In this case, it is assumed that the additional error has a normal distribution. Because the scheduling run is scheduling generation for the next 24 hours only, the hydropower model is most sensitive to the day-ahead forecast and much less so to the characteristics of forecasts with longer lead times.

\subsection{Test Case}

\subsubsection{Physical Hydropower System}

The described methodology is applied to a test case system of five hydropower plants on a single river with a combined nameplate capacity of approximately $5000 \mathrm{MW}$ and an assumed maximum load of 4000 MW. A single inflow to the system is modeled. The cascading reservoir system has limited storage, approximately two days of typical flow, but the size of the individual reservoirs varies significantly from 12,760 acre-feet to 168,720 acre-feet. This variation in storage capacity impacts how the system must be operated. For example, Reservoir 1D, the third power reservoir in the system, has very little storage and is therefore operated essentially as a run-ofriver hydropower plant. For this reason, it is excluded from providing ancillary services. All reserves, therefore, must be provided by the remaining four hydropower projects. A depiction of the RiverWare model is shown in Figure 4.2.

\subsubsection{Policy}

The policy set for the hydropower system has a general priority order that sets licensing constraints (minimum and maximum pool elevations) at the highest priority. These are followed by non-power water and environmental policy objectives and then the power objectives to meet load and reserve requirements and to maximize the economic value of energy and ancillary services. A summary of the policy is provided here. 


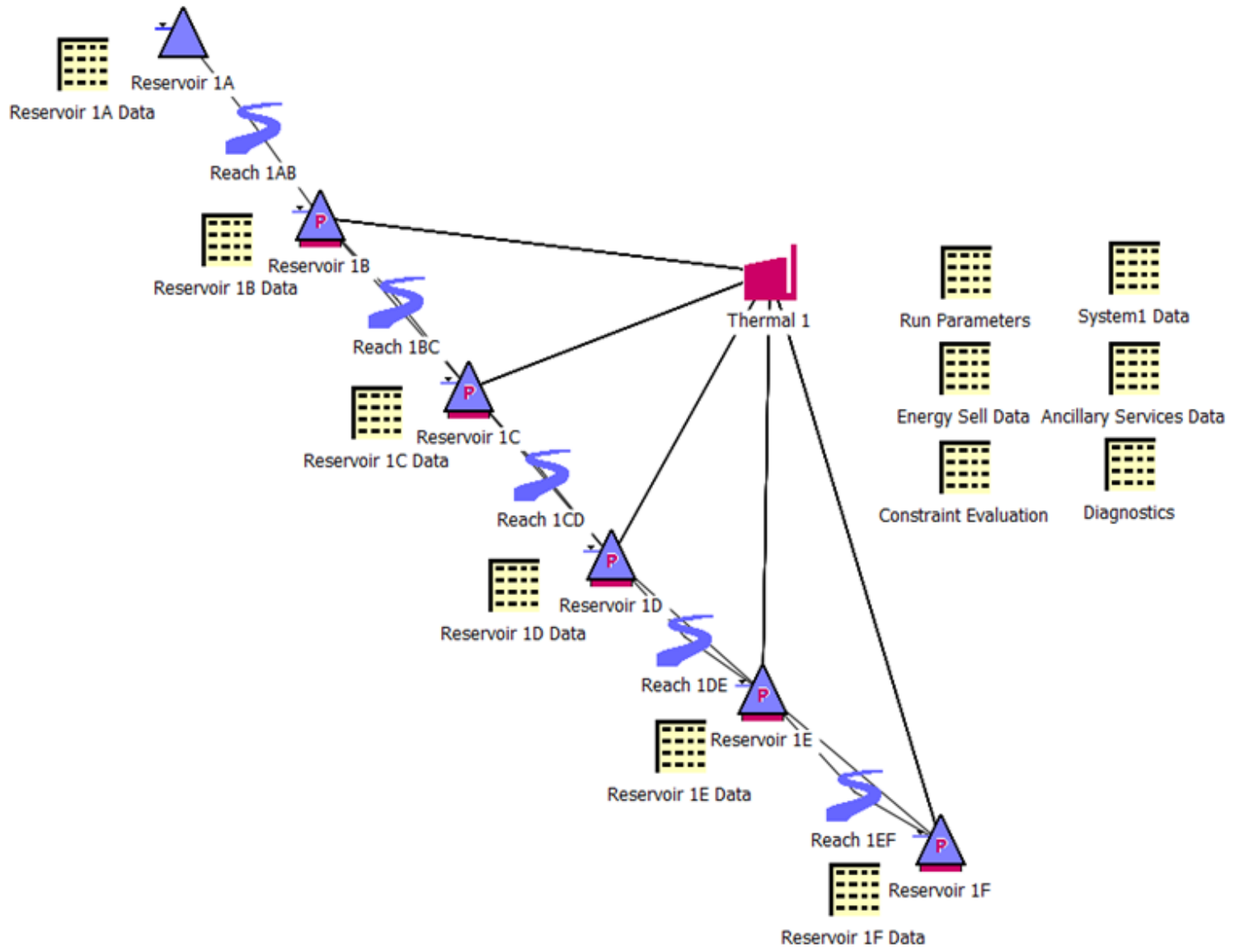

Figure 4.2: RiverWare model workspace showing 5 hydropower projects in a cascading reservoir system; Reservoir $1 \mathrm{~A}$ is a storage reservoir and is included here only to provide the inflows to the hydropower system.

\subsubsection{Non-power Policy}

In reality, each hydropower system has non-power policies unique to that system that limit its operations. While the specific policies vary from one system to another, the common result in regards to hydropower operations is that the non-power policy, in general, reduces the flexibility with which the hydropower system can be operated to meet power objectives. The non-power policy implemented in our model is representative of policy constraints in the Pacific Northwest, though it is not the actual policy of any individual system. Constraints are based on policies such as those coming from biological opinions and other agreements in place primarily to improve fish 
survival rates in the hydropower system. Examples of constraints in the policy set include:

- Minimum flow requirements at all reservoirs with higher minimum flow requirements which vary seasonally for the final downstream reservoir.

- Minimum spill requirements at all reservoirs in the spring and summer to improve fish passage.

- Maximum spill limits at all reservoirs used as a proxy for limits on total dissolved gas (TDG) concentration.

- Limits, which vary seasonally, on the difference between the daily minimum and maximum outflow at the final downstream reservoir.

- Limits on the hourly change in tailwater elevation at the final downstream reservoir.

- Meeting target ending storage levels.

- Minimum pool elevations for recreational purposes at the two largest reservoirs. (Note that this constraint actually comes at a priority below meeting local load and reserve requirements but before the objective to maximize the value of energy and ancillary services sold into the market.)

A number of the policy constraints apply to the final reservoir, $1 \mathrm{~F}$, which must re-regulate the flow passing through the hydropower system. This is typical of many hydropower systems. Due to the essentially run-of-river nature of this system, however, constraints on this reservoir have impacts on operations throughout the system.

\subsubsection{Power Policy and Reserve Requirements}

Power-related policy includes constraints to meet load, reserve (ancillary services) requirements and minimum generation requirements for station service at each reservoir. The operations run also includes additional soft constraints to use the power schedules for each hydropower plant 
from the scheduling run within the tolerances of the scheduled load following reserve and regulation capacity. The use of soft constraints represents the flexibility of the hydropower system to adjust operations to meet real time conditions. The minimax solution, however, will force the actual generation to be as close to the schedule as possible while meeting all higher priority constraints. This prevents the hydropower system from completely re-optimizing during the operations run to meet the new load and thus represents the loss of efficiency that generally occurs when the system must deviate from its schedule to meet changing conditions.

Increased balancing reserve requirements are typically seen as the primary source of wind integration costs, and thus the determination of reserve requirements is a key factor in wind integration studies. Ela et al. (2010) provide a summary of how balancing reserves have been calculated in previous wind integration studies. The requirement for regulation capacity, both up and down, used in our model is based on the method used in the Eastern Wind Integration and Transmission Study (EnerNex, 2010). The regulating reserve requirement is set to $1 \%$ of load which is assumed to be approximately three standard deviations of the one-minute variability separated from the 20minute rolling average. Wind variability is assumed to be insignificant at this timescale and thus does not contribute to the regulation capacity requirement. ${ }^{4}$ For base scenarios the contingency reserve requirement is set to $500 \mathrm{MW}$ which is assumed to represent the portion of the single largest contingency in the BA that must be covered by the hydropower system. A minimum of $50 \%$ of the contingency reserve must come from spinning reserve (WECC, 2008). In alternative, scenarios contingency reserve requirements of $250 \mathrm{MW}$ and $1000 \mathrm{MW}$ are modeled.

In our model, all increases in reserves due to wind are incorporated into the load following reserve requirement. Load following reserve is scheduled to assure there is sufficient capacity, both up and down, to balance longer time scale variability in net load and net load forecast error. Assuming that these two components of load following are uncorrelated, the combined requirement is calculated by taking the root sum of squares of the individual requirements. For

\footnotetext{
${ }^{4}$ The EWITS also includes a term for wind forecast error in the regulating reserve requirement. In our model all increases in reserves due to wind forecast error are incorporated into the load following reserve.
} 
the variability component, the requirement is set equal to the absolute value of the hourly net load change corresponding to an exceedence probability of 0.995 (see Acker et al., 2012b; Holttinen et al., 2008; Robitaille et al., 2012) and is evaluated for each calendar month. The forecast error component is similar. In the absence of empirical forecast data, one hundred traces of day-ahead load and wind forecasts are generated for each month using the synthetic forecast generators described in Sections 3.2 and 2.4.3.1. These are then combined into net load forecasts, and the reserve requirement is calculated as the absolute error magnitude associated with an exceedence probability of 0.995 . Alternative scenarios are tested using exceedence probablilities of 0.99 and 0.98 . The load following reserve requirement is removed in the operations run to simulate the use of the reserve capacity to meet the actual load as it deviates from the forecast.

$$
\begin{gathered}
L F_{S, t}^{r e q}=\sqrt{\left(L F_{N L \Delta}^{r e q}\right)^{2}+\left(L F_{N L F E}^{r e q}\right)^{2}} \\
P\left(\left|L_{S, t}^{n e t}-L_{S, t-1}^{n e t}\right| \leq L F_{N L \Delta}^{r e q}\right)=0.995 \\
P\left(\left|\epsilon_{t}^{L}+\epsilon_{t}^{W}\right| \leq L F_{N L F E}^{r e q}\right)=0.995
\end{gathered}
$$

The economic objective comes at the final priority. For the scheduling run the objective is to maximize total revenue, the combined value of day-ahead energy and ancillary services (capacity and deployment energy value) after meeting all non-power constraints, load and reserve requirements and target ending storages. In addition to the aforementioned constraints, this objective is also subject to a static transmission constraint which limits the combined energy and ancillary services that can be sold into the market. The transmission constraint applies only to energy and ancillary services being sold into the market. No transmission constraints are modeled within the hydropower system. The transmission capacity for the baseline scenarios is $1500 \mathrm{MW}$. Alternative scenarios are also modeled to test the effects of increased transmission capacity.

The objective for the operations run is to maximize the ending energy in storage value after meeting all power and non-power constraints and meeting minimum ending storage targets at each reservoir. The energy in storage metric represents the generating potential of a unit of water as it passes through all downstream hydropower plants and thus gives a higher weight to water stored 
farther upstream in the system. Alternative scenarios implement a real-time energy market in the operations run, and in these cases the objective is to maximize the value of energy sold into the real-time market.

\subsubsection{Scenarios}

The impacts and value of integrated wind generation and hydropower are dependent on a large number of factors and can vary significantly with seasonal conditions. Many previous wind integration studies run scenarios for a complete year or multiple years often to arrive at an average cost of wind integration. The approach we present here is to model specific weeks to evaluate the sensitivity to varied conditions and system parameters. This allows the effects of specific seasonal conditions to be analyzed.

\subsubsection{Base Scenarios: Full Policy}

Three weeks are selected which have different hydrologic and wind conditions as well as having different seasonal non-power constraints in effect. These are modeled with the full policy set in place for the Base scenarios.

\section{Spring Scenario: May 14-20, 2010}

The first scenario is intended to model relatively normal stream flow. A minimum spill requirement for fish passage is in effect for three of the five reservoirs. A seasonal minimum environmental flow of $65 \mathrm{kcfs}$ is required at the final reservoir, $1 \mathrm{~F}$. The daily outflow fluctuation at Reservoir $1 \mathrm{~F}$ is limited to $20 \mathrm{kcfs}$.

\section{Summer High Flow and High Wind Scenario: June 22-28, 2010}

The second scenario represents a high flow scenario with high wind generation, often one of the critical operating conditions for hydropower systems with integrated wind generation. The excess water flowing through the system can increase the need to run the hydropower plants near capacity, which can reduce the flexibility for balancing wind generation. While this scenario is constrained by physical conditions, it is the least restricted by non-power policy constraints. Minimum spill 
requirements are in effect for all five reservoirs. The maximum allowable spill for TDG limits is increased to $100 \mathrm{kcfs}$ at each dam (75 kcfs at Reservoir 1D). The maximum allowable daily outflow fluctuation at Reservoir $1 \mathrm{~F}$ is increased to $40 \mathrm{kcfs}$.

\section{Fall Scenario: October 30-November 5, 2010}

The third scenario is marked by a restricted outflow range at Reservoir $1 \mathrm{~F}$ during daylight hours (55 kcfs-70 kcfs) to protect spawning salmon. This constraint affects scheduling throughout the system. This is also the most water limited scenario.

\subsubsection{Simplified Policy Scenarios}

In order to evaluate the impact of modeling non-power policy, the Base scenarios are compared to the same scenarios with two levels of simplified policy sets. The only non-power constraints in effect for the Unconstrained case are minimum and maximum pool elevations, constant minimum outflows for all reservoirs and target ending storages. The Moderate Policy case adds minimum spill and seasonal environmental flow requirements. All power-related constraints and objectives are in place for all policy cases.

\subsubsection{Isolated Effects of Wind Forecast Error, Wind Variability and Wind Energy}

The effects of adding wind generation to the power system are divided into three components: energy, "predictable" variability and forecast error. In general added wind energy is considered to have a positive impact while variability and forecast error have negative impacts, though in certain cases the opposite may be true in the short term. In order to evaluate the isolated effects of these components each Base scenario is also run without wind forecast error and then without wind variability. All other inputs, including load forecast error, remain the same as the Base case. For the No Variability case, rather than model the added wind energy as a constant flat block, the equivalent amount of wind energy over the one-week scenario is shaped to approximately follow the load in a regular diurnal pattern with a ramping rate approximately equal to that of a coal plant. This represents a generating resource that is able to add energy to system when, in general, it is 
beneficial. Comparing the No Forecast Error case with the Base case with forecast error isolates the impacts of wind forecast error, comparing the No Variability case with the No Forecast Error case isolates the impacts of predictable wind variability, and comparing the No Variability case with the No Wind case isolates the effects of wind energy.

\subsubsection{Alternative Scenarios for Sensitivity Analysis}

In recognition of the fact that wind integration effects are specific to each system and scenario conditions, a sensitivity analysis is carried out on select conditions and parameters within the scenarios described above. A description of specific characteristics that are evaluated in the sensitivity analysis is given in Section 4.5.3.

\subsubsection{Input Data}

In order to create realistic scenarios, input data for load, wind, prices and inflows are based on synchronized actual observed data from select weeks in 2010 as described here. Inflow data are taken from Grand Coulee Dam hourly outflow data. ${ }^{5}$ This represents the fact that operators of run-of-river hydropower systems often have to manage variable flows resulting from releases from upstream hydropower projects. Load data are based on Mid-Columbia hourly combined non-federal request data. The Mid-Columbia request data display greater intra-hour variability than typical load for a BA, so a five hour rolling average is applied to smooth the data. This results in a load profile that is more representative of a typical load. The data are scaled for different scenarios to evaluate the sensitivity to various levels of local load. Synthetic load forecasts are generated using an $\operatorname{ARMA}(2,1)$ model for the day-ahead forecast similar to the synthetic wind forecast. For the load, however, forecast error is assumed to be normally distributed with a mean of zero. The day-ahead MAPE for forecast error is $2.5 \%$, a typical value for load forecast error (Taylor and McSharry, 2007).

The initial market clearing prices for energy and ancillary services are taken from observed

\footnotetext{
${ }^{5}$ http://www.nwd-wc.usace.army.mil/ftppub/water_quality/10data/
} 
CAISO MCPs. ${ }^{6} 7$ Day-ahead hourly prices correspond to the same time periods in 2010 as the load, wind and hydrologic data. For the scenarios which include a real-time energy market, the average hourly real-time MCPs from the same dates and hours in 2008 are used. ${ }^{8}$ These initial data are then used to generate a price curve at each time to represent the market depth for each product. For day-ahead energy, the prices are set for $50 \mathrm{MWh}$ blocks and the modeled MCP decreases by $1 \%$ for each additional $50 \mathrm{MWh}$ sold based on an approximation of results observed by Jónsson et al. (2010). The real-time market is assumed to have less depth than the day-ahead market, and thus the reduction factor is set to $5 \%$ per $50 \mathrm{MWh}$. The prices for ancillary services are based on $10 \mathrm{MW}$ blocks, with a reduction factor of $4 \%$ per $10 \mathrm{MW}$ for regulation down and 5\% per $10 \mathrm{MW}$ for regulation up, spinning reserve, and non-spinning reserve. The price curve for a given product may vary considerably from one market to another particularly dependent on the mix of available generation resources and their associated marginal costs. The sensitivity to the price curve parameters for the day-ahead and real-time energy markets are tested in alternative scenarios.

\subsection{Discussion of Results}

\subsubsection{Impact of Non-power Policy}

This section describes the effects of non-power policy on the behavior of the integrated hydropower and wind system as observed in the model results. First the effects on the total economic value of the system are analyzed for the three different seasonal hydrologic scenarios. Results indicate that the impacts of accounting for non-power policy in wind and hydropower integration analysis can vary substantially, even within a single system, depending on the scenario conditions. Model outputs demonstrate how complex interactions in the integrated system can cause a nonlinear response to increased wind penetration. This is followed by an assessment of how wind

\footnotetext{
${ }^{6}$ http://www.caiso.com/market/Pages/ReportsBulletins/ReportsBulletinsArchive/Default.aspx

7 http://oasis.caiso.com/mrtu-oasis $/$ ?doframe=true\&serverurl=http $\% 3 a \% 2 f \% 2 f f r p t p 09 \% 2$ eoa $\% 2$ ecaiso $\% 2$ ecom $\% 3$ a8000\&volume $=$ OASIS

${ }^{8}$ http://oasishis.caiso.com/index.jsp
} 
integration affects the ability of the hydropower system to satisfy all policy constraints.

\subsubsection{Economic Outputs}

Figure 4.3 shows the total system value for each scenario at the three different policy levels. Results shown are the mean values from running 10 traces of wind forecasts for each scenario. In the spring scenario the non-power policy has a very noticeable effect, most prominent at $30 \%$ penetration. The large difference in value between the Unconstrained and Moderate Policy cases is primarily due to the inclusion of the minimum spill requirement in the Moderate Policy. When the spill requirement is removed the water is available for increased generation. The differences between the Moderate Policy and Full Policy cases are due to reductions in system flexibility due to more stringent flow constraints, primarily at Reservoir $1 \mathrm{~F}$, where the most stringent flow restrictions apply. These flow constraints reduce the amount of energy sold in two manners. First, inclusion of non-power policy constraints in the calculation of hydro capacity directly limits both the energy that can be generated and the reserves that can be provided by Reservoir $1 \mathrm{~F}$. This means that more reserve capacity must be provided by upstream reservoirs reducing their generating potential. Second, due to the run-of-river nature of the system, the flow requirements at Reservoir $1 \mathrm{~F}$ have a strong influence on the timing of flows throughout the entire system reducing the overall system flexibility. (An example of this type of interaction is illustrated in Figure C.4 in Appendix C.) This effect would not be captured without modeling both the hydraulic relationships of the complete hydropower system and its non-power constraints.

The Full Policy case for the spring scenario demonstrates a complex interaction between the physical system and policy constraints showing a decrease in total system value from $20 \%$ to $30 \%$ penetration and then an increase again at $40 \%$ penetration. The dip in total value at $30 \%$ penetration corresponds to a reduction in the amount of day-ahead energy sold into the market (see Figure 4.4). At this level of wind, the load following requirement becomes large enough that scheduled hydropower generation must be reduced in order to maintain sufficient reserves. The occurrence and magnitude of the decrease in value are highly sensitive to assumptions about the 

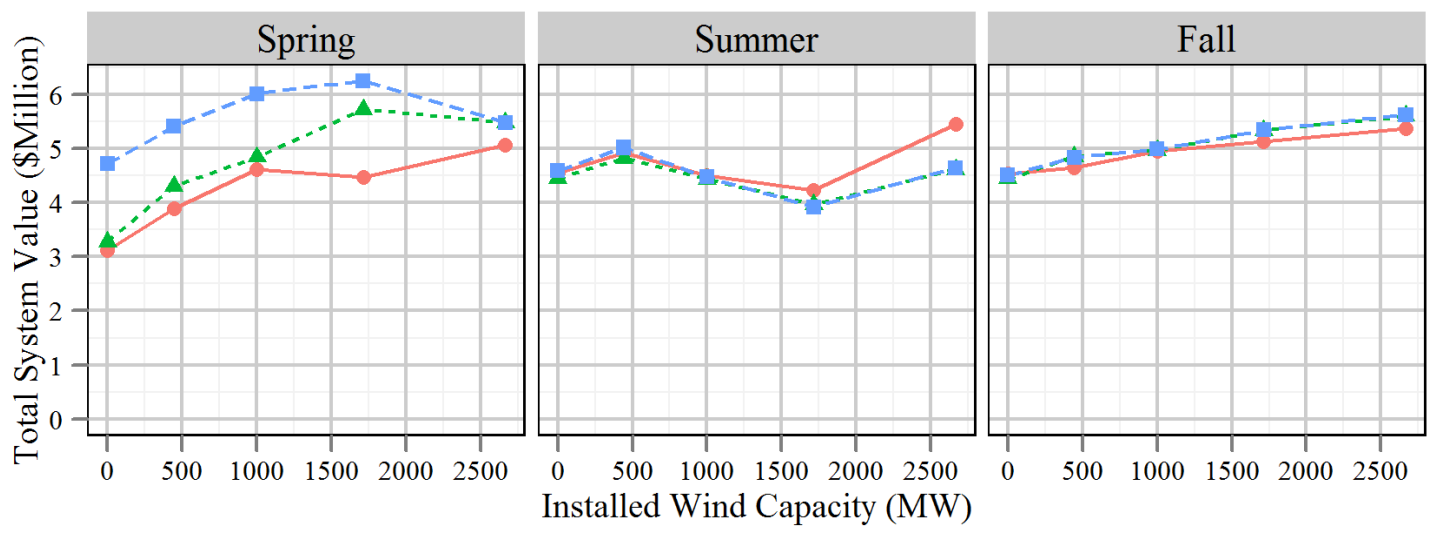

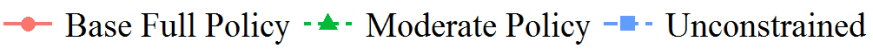

Figure 4.3: Total system value for full policy and simplified policy cases, 3 scenarios; data points correspond to No Wind, $10 \%, 20 \%, 30 \%$ and $40 \%$ penetration

scheduling of hydropower generation and load following reserves as well as market structures which are analyzed in Section 4.5.3. In the Unconstrained and Moderate Policy cases, this same decrease in value is not observed until $40 \%$ wind penetration. When the non-power constraints are relaxed, the system has more flexibility to provide the necessary reserves at $30 \%$ penetration. There is essentially no difference in value in the reduced policy cases in the $40 \%$ wind case. At this level of wind penetration, the load following requirement limits generation in the Unconstrained case even without the spill requirement in place.

In order to explain some of the results, including the increase in total system value observed at $40 \%$ wind penetration, we first explain a complex policy interaction present in many systems that is reflected in the model. In certain conditions system operators may be required to meet a high priority constraint that has an effect of actually improving a low priority objective. For example, the need to maintain spill below a maximum allowable limit may force more water to be sent through the turbines. This results in increased generation which leads to more revenue. This may come at the expense, however, of violating a medium level constraint. In this case, the 
increased turbine release forces the generating units to operate closer to their maximum output, in which case they may not maintain sufficient reserve capacity. Without the high priority spill constraint, the system would meet the reserve requirement and actually generate less. With the spill constraint in place, the system gets the side benefit of increased generation but at the expense of violating the reserve requirement.

Utilization of goal programming makes it possible to model such operational conditions and interactions of policy. In goal programming, the addition of a high priority constraint can cause a medium priority constraint to be relaxed or further relaxed. Together, the feasible region may be reduced in some places but increased in others. Thus a low priority objective function may either increase or decrease as a result of this change. In this sense, goal programming has an apparent paradox that adding high priority constraints can improve a low priority objective function. This is not really a paradox because the change also increases violation of a medium priority constraint and reduces the desirability (or utility) of the solution.

The increase in total system value observed at $40 \%$ wind penetration in the spring Full Policy scenario is an example of when the requirement to meet a high priority environmental constraint has the side effect of improving the economic objective. In this case, the high priority constraint is the maximum spill limit for TDGs, and the medium priority constraint is the load following reserve requirement. The reservoirs are full and already spilling at the constraint limit. All additional water must pass through the turbines. This violates the load following reserve requirement because the additional water forces the units to generate closer to capacity. Sufficient reserve capacity is not maintained. This benefits the low priority economic objective because more energy is being generated, which can then be sold. This comes at the expense, however, of violating the load following reserve requirement.

In the summer Full Policy case a reduction in total value is observed at $20 \%$ wind penetration, as opposed to $30 \%$ in the spring scenario. The higher flows in the summer scenario result in excess energy in the system even before wind generation is added. The load following reserve requirement again limits any additional generation. It is not immediately evident if the changes in system 
behavior between the spring and summer scenarios are due primarily to the different hydrologic and wind conditions or simply due to different energy prices. In order to evaluate what is driving the different system behavior, an alternative summer scenario is run using the spring price inputs. Results show slight differences in total system value with the different prices, but total hydro generation, spill and the average value of the added wind generation are the same as in the case with the actual summer prices. This indicates that the changes in system behavior are primarily due to the hydrologic and wind conditions. Results from these two scenarios can be seen in Figure C.14 in Appendix C.

In the summer scenario the Full Policy case results in a higher total value than the reduced policy cases for $30 \%$ and $40 \%$ wind penetration. This is another example where meeting a high priority constraint has a side effect of improving the economic objective. Once again the TDG constraint forces more water through the turbines at the expense of violating the load following requirement. In this scenario, an increase in value at $40 \%$ wind is observed even in the reduced policy cases. In the reduced policy cases the high priority constraint causing this effect is the physical maximum spill limit. The system violates the load following requirement as opposed to exceeding the spill capacity.

In the fall scenario, added wind capacity continues to add value to the system even at high penetrations. (Note while the installed capacity is the same across all scenarios for a given penetration, the actual wind generation in the fall scenario is approximately one-third of the spring and summer scenarios.) In the Full Policy case, non-power constraints reduce the total economic value from the reduced policy cases at high wind penetrations when greater flexibility is required.

In Figure 4.4, the total values from the spring and fall Base cases are divided into their three individual components: day-ahead energy value, ancillary services value and the future value of energy in storage. The future value of energy in storage refers to the potential revenue that can be generated with the water that is stored in the reservoirs at the end of the run. This quantity is referred to hence forth as the energy in storage value. In the spring scenario the largest contributor to differences in the total value with changing wind penetration is the day-ahead energy. Similar 
results are observed for the summer scenario with the total value curve following the general shape of the day-ahead energy value curve. In the fall scenario, day-ahead energy revenue increases at low penetrations, then decreases at $30 \%$ wind, similar to the spring scenario. There is less excess energy in the water-limited fall scenario, however, so the decrease in value is not as sharp. The corresponding increase in energy in storage value results in a net increase in total system value even at high penetrations. One assumption that should be noted here is that the unit value of energy in storage does not vary with wind penetration. Conclusions about the relative contribution of energy in storage to the total value, therefore, should be treated with some caution.

While ancillary services make a smaller contribution to total value than day-ahead energy, they still provide approximately $20 \%$ of the total revenue in the spring No Wind case. This value goes to nearly zero by the $30 \%$ case. The need for more reserve capacity to meet the local requirements with higher wind penetration leaves no excess capacity to bid into the ancillary services market. It is important to note that all of the ancillary services revenue is being provided by the hydropower system, whereas all of the additional energy revenue beyond the No Wind case is assumed to come from wind generation.

In summary, non-power policy can have a significant impact on the economic value of integrated wind and hydropower. The relative impact of policy constraints, however, is dependent on seasonal conditions. Complex interactions between the physical system and the policy governing operations can have the effect of both benefitting and reducing the economic outputs. Limitations on system flexibility can reduce revenue, but at times the need to satisfy certain high priority policies, such as maximum spill limits for TDGs, can result in improved economic benefits by forcing more generation. This may come at the expense, however, of violating medium priority constraints.

\subsubsection{Constraint Satisfaction}

In addition to the economic outputs, the model results provide information on how wind integration impacts the ability of the hydropower system to satisfy all power and non-power policy constraints. For power-related constraints, it was mentioned previously that high wind penetra- 


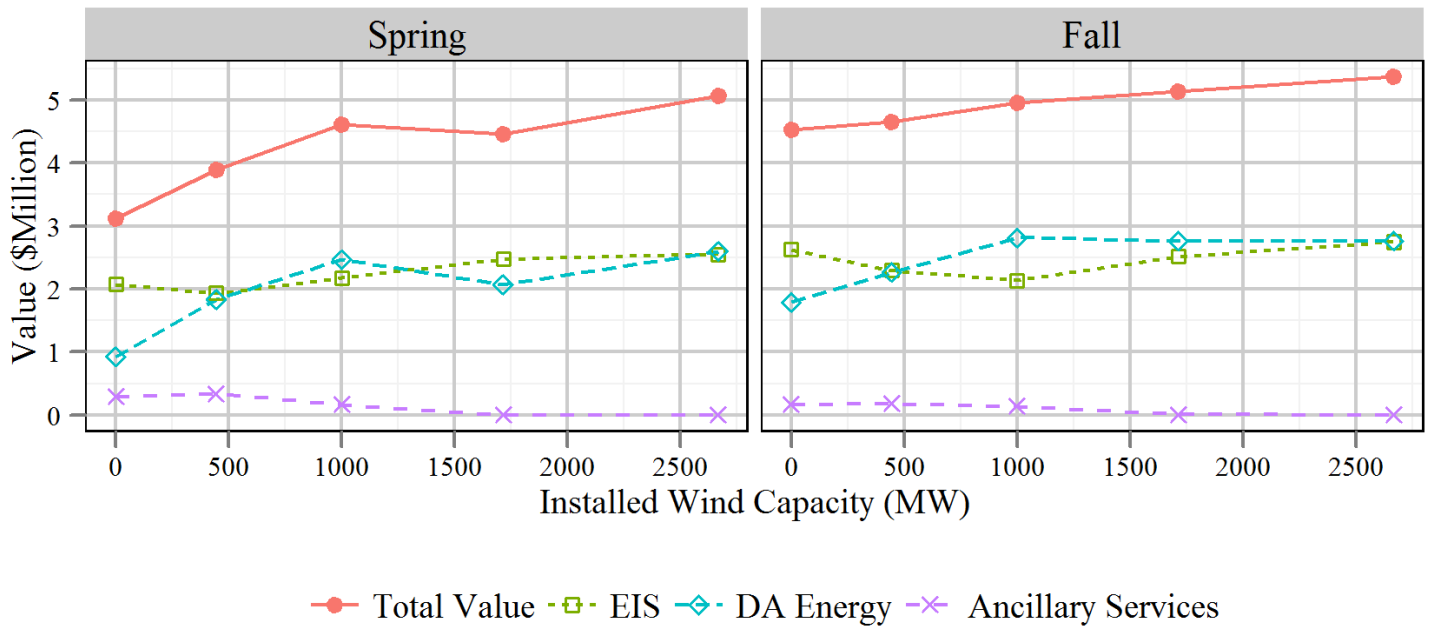

Figure 4.4: Components of the total system value for the spring and fall base scenarios: total value, ending energy in storage value, day-ahead energy value, and ancillary services value

tion causes violations of the load following requirement. Additionally, higher wind penetration is observed to increase the number of hours with unmet load in the spring and fall scenarios. The number of violations are shown in Appendix C in Figure C.6. In the fall scenario there are no hours with unmet load in the No Wind Case and an average of 12 and 15 hours with unmet load for the $20 \%$ and $30 \%$ wind cases respectively. In the spring scenario, the $20 \%$ case has an average of 6 hours when the hydropower system cannot meet its scheduled load. Analysis shows that these periods of unmet load are due to wind forecast error. The same violations do not occur for the same scenarios with perfect wind forecasts. The summer scenario shows no difficulties in meeting load, other than the $40 \%$ wind case, but the regulation down requirement is violated an average of 6 hours for the $20 \%$ case, 46 hours for the $30 \%$ case, and 57 hours at $40 \%$ wind. In this scenario, excess water in the system prohibits any reduction in generation to provide regulation down because it would lead to further violation of the TDG limits. Unmet regulation down is also seen in the spring scenario at $40 \%$ wind.

Violations of non-power constraints are shown in Figure 4.6. Occasional violations of the 


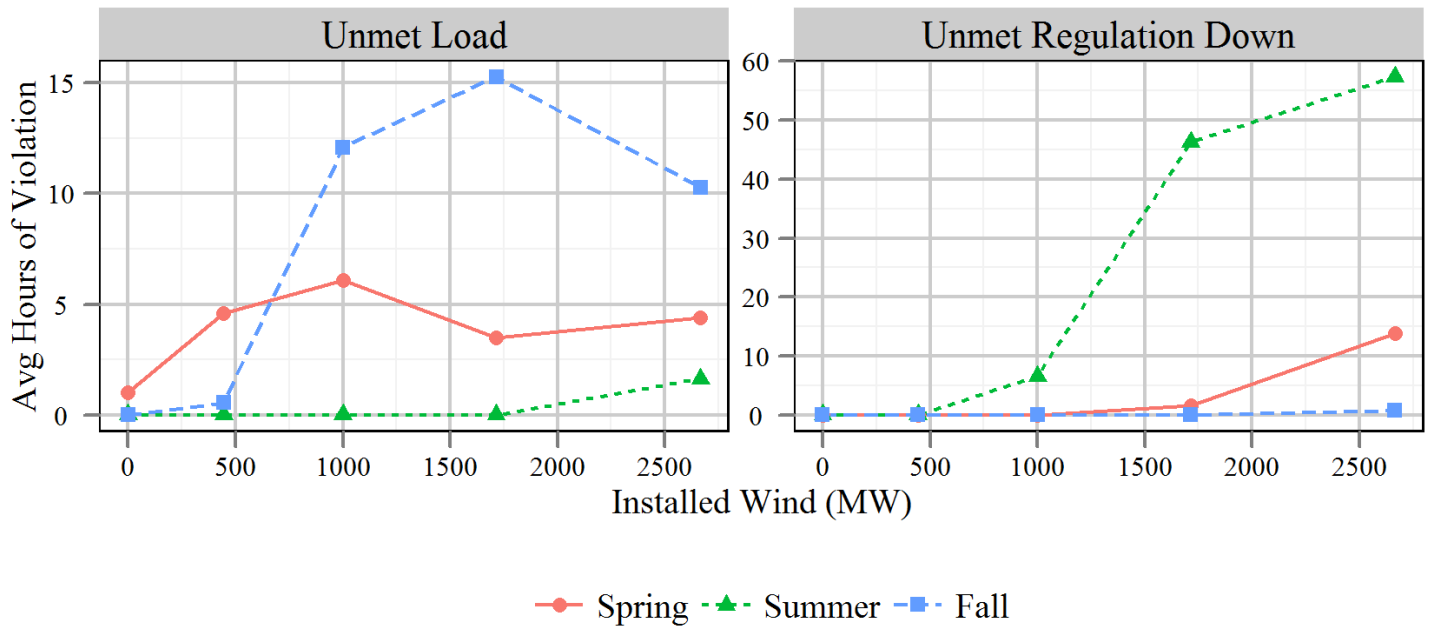

Figure 4.5: Power-related constraint violations: Unmet load and unmet regulation down capacity for spring, summer and fall base case scenarios

minimum flow requirements at Reservoir $1 \mathrm{~F}$ are observed for the spring and fall scenarios with an average of just over 2 hours with violations in the $30 \%$ case. In the summer high flow scenario, the spill limit for TDGs is the primary constraint that causes difficulties for system operations. Beginning with the $20 \%$ wind case occasional violations occur. This increases to 29 hours over the spill limit at all reservoirs with $30 \%$ wind and 48 hours at $40 \%$ wind. These violations of TDG constraints tend to correspond to periods of negative forecast error as illustrated in Figure 4.7. When more actual wind arrives than what is forecast, the reservoirs, which are already full, are forced to spill beyond the allowable limit. 


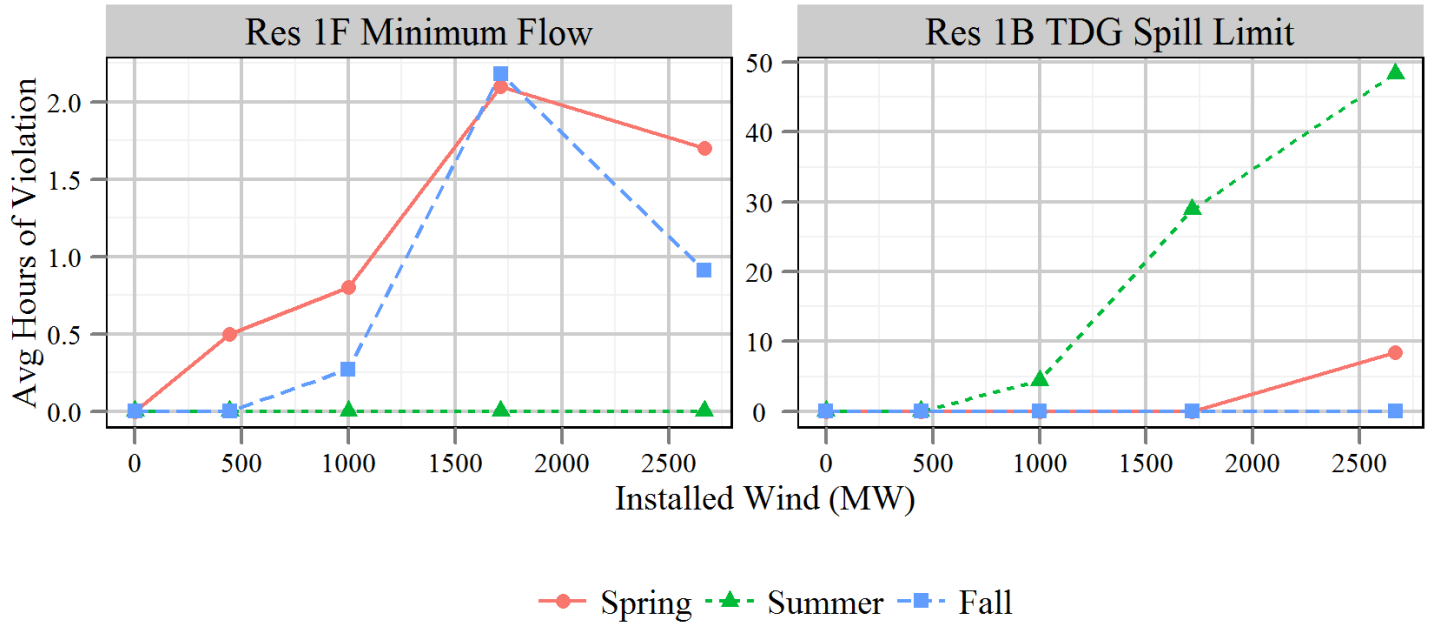

Figure 4.6: Non-power constraint violations: Reservoir 1F Minimum flow requirement and Reservoir 1B maximum spill limit for TDGs (TDG spill violations are similar for the other reservoirs.)
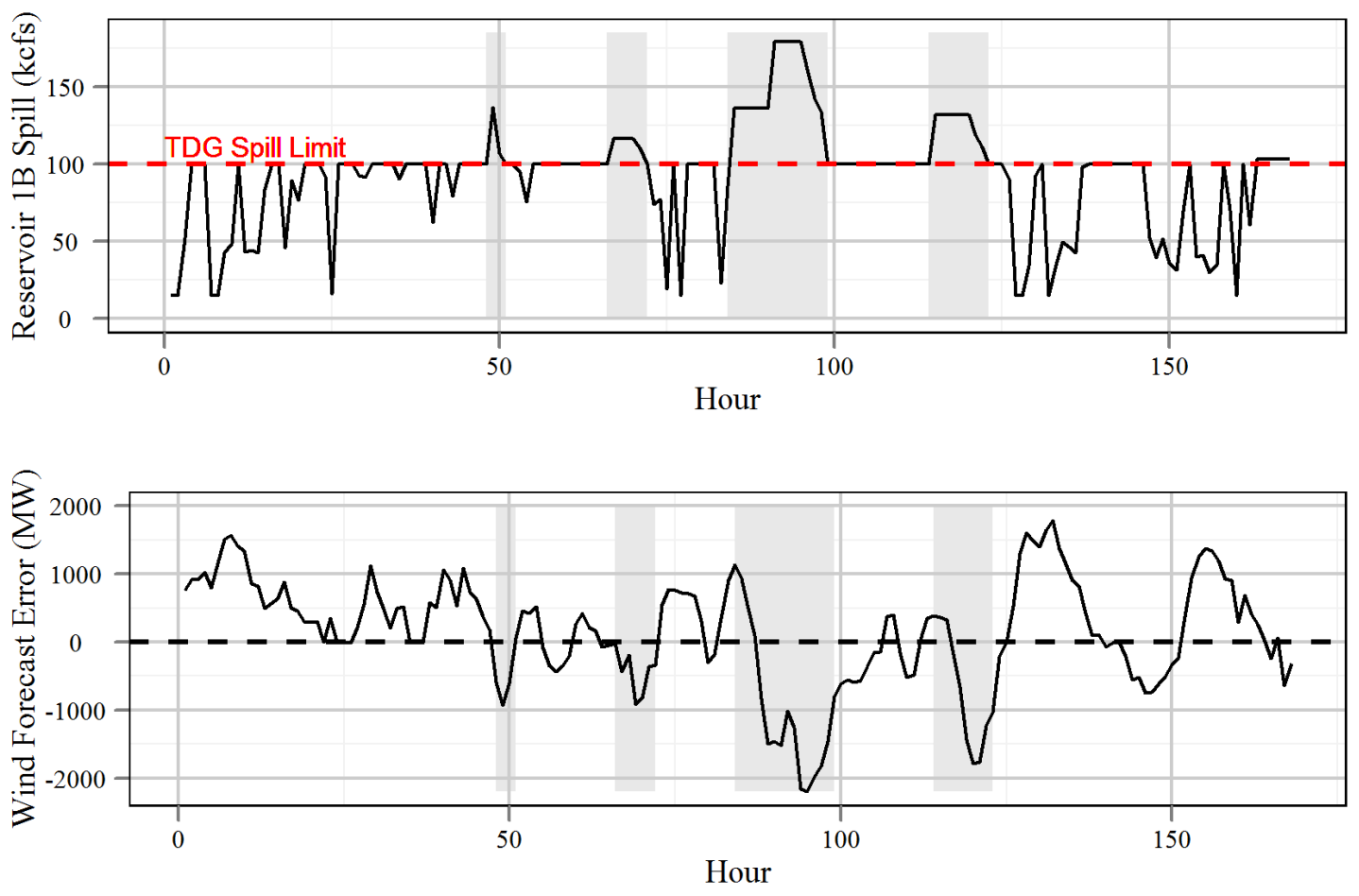

Figure 4.7: Reservoir 1B spill and wind forecast error for a single forecast trace from the summer scenario, $40 \%$ wind penetration, showing periods of spill exceeding the TDG limit corresponding to periods of negative wind forecast error 


\subsubsection{Isolated Effects of Wind Forecast Error, Variability and Energy}

Average total system value for the spring Base case and for the No Wind Forecast Error and No Wind Variability cases are shown in Figure 4.8(a). Figure 4.8(b) shows the resulting isolated value of each wind component: energy, variability and forecast error. In general, as expected, removing forecast error and variability have a beneficial impact on total system value. This comes primarily from removing the load following reserve requirements associated with wind variability and wind forecast error. Results from running the No Error and No Variability cases with the full load following reserve requirement display total values very similar to the Base case. (Additional sensitivities to the load following reserve requirement are examined in the following section.) The largest impact is due to wind forecast error. This can be observed clearly in the plot of spill (converted here to the equivalent energy) for the Base, No Forecast Error and No Variability cases (Figure 4.8(c)). All additional spill in the Base case beyond that in the No Error case is the result of wind forecast error and its associated reserve requirement.

In some cases, the value of additional wind capacity decreases even when the negative contributions of variability and forecast error are removed. When the isolated positive contribution of wind energy is plotted as the average value per MWh of wind generation (Figure 4.8(d)), it shows that the value of added wind energy varies depending on scenario conditions. Note that the wind energy value shown in Figure 4.8(d) is not the complete wind generation value. It is the isolated value of the positive contribution of the energy component with the negative components of variability and forecast error removed. In the spring scenario, which is more energy limited, the average unit value of wind energy remains relatively constant at high levels of wind penetration dropping only slightly at $40 \%$ penetration. In the summer, high-flow scenario, which has excess

energy in the system, the average value of wind energy decreases significantly as the amount of installed wind increases. 
(a)

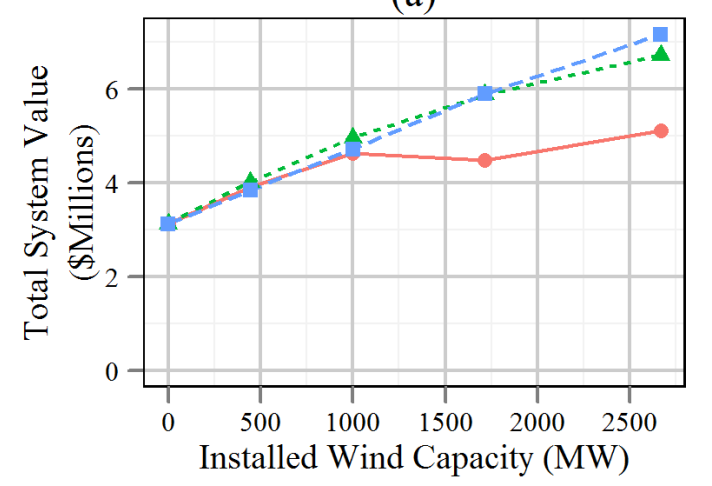

$\rightarrow-$ Base - - No Error - - No Variability

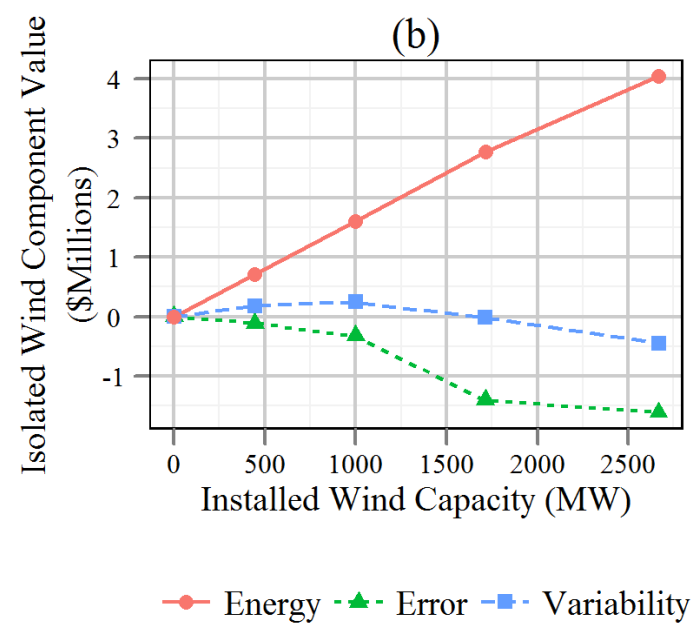

(b)

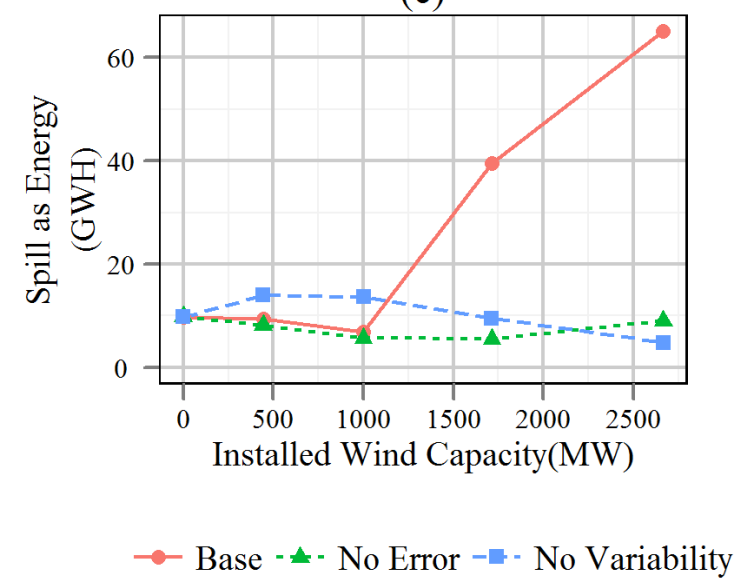

(d)

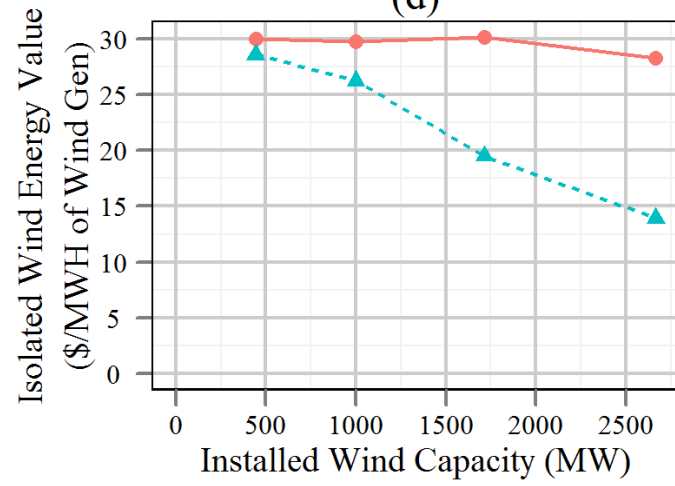

$\multimap$ Spring -- Summer

Figure 4.8: Effects of isolated wind energy, variability and forecast error, spring scenario: (a) Total system value for runs with full wind (Base), wind with no forecast error and wind energy with no variability; (b) Isolated value of wind energy, variability and forecast error; (c) Spill converted to equivalent energy for Base, No Forecast Error and No Variability cases; (d) Average value of isolated wind energy (with forecast error and variability effects removed) per unit of generation for spring and summer scenarios

\subsubsection{Sensitivities to System Characteristics}

The results presented above suggest that the hydropower system response to wind integration and the total value of the system are heavily dependent on assumptions regarding the scheduling of 
generation and reserves. A primary assumption in the reserve requirement calculation is that the hydropower system is scheduled based on day-ahead forecasts, and that schedule is not updated prior to operations. This requires the load following reserves to be sufficient to cover all likely wind forecast error (exceedence probability of 0.995) for a 24-hour lead time. Wind forecast error decreases significantly for lead times less than eight hours (Acker, 2011a), so allowing the generation schedule to be adjusted with updated wind forecasts could allow for a reduction in the load following requirement in the shorter scheduling time frame. This may result in improved efficiency in the hydropower system; however, with the economic value in the model coming entirely from the dayahead market, the value of this improved efficiency would likely not show up significantly in the short term. The energy committed in the day-ahead market is still based on the day-ahead forecast and would have to account for the day-ahead forecast error. Allowing energy to be sold in the real-time market, however, makes use of the additional capacity freed up with the reduction of the load following reserve requirement in the short term when the wind forecast error is reduced. This is tested by approximating a real-time market by hourly average prices and energy. (Actual real-time markets in the U.S. clear every five to fifteen minutes (Milligan et al., 2009).)

Real-time market price data were not available for the dates corresponding to the scenarios modeled in our study. Instead prices from the same days in 2008 are used. There was also a lack of reliable data on which to base the market depth parameters. Instead, two different market depth cases are tested. The RT High and RT Low cases modeled here correspond to 5\% and $10 \%$ reductions in real-time prices for every additional $50 \mathrm{MWh}$. Due to the lack of reliable real-time price data, the real-time market is included as an alternative scenario in the sensitivity analysis as opposed to being included in the base scenarios.

The inclusion of a real-time market in the operations run results in improved total system value at high wind penetrations as illustrated in Figure 4.9(a). This is consistent with previous wind integration research (see Milligan et al., 2009; Acker et al., 2012a). The results indicate that the added value is sensitive to assumptions about the depth of the real-time market. The availability of the real-time market also reduces spill (here converted the equivalent energy) to approximately the 
same level as the No Wind case as seen in Figure 4.9(b). It is necessary to clarify that in the Base case without a real-time market generation must meet and not exceed the load in the operations run. The power grid cannot absorb extra energy. The real-time market provides the opportunity to make use of extra capacity in the operations run allowing more water to be passed through the turbines when the reservoirs are full.
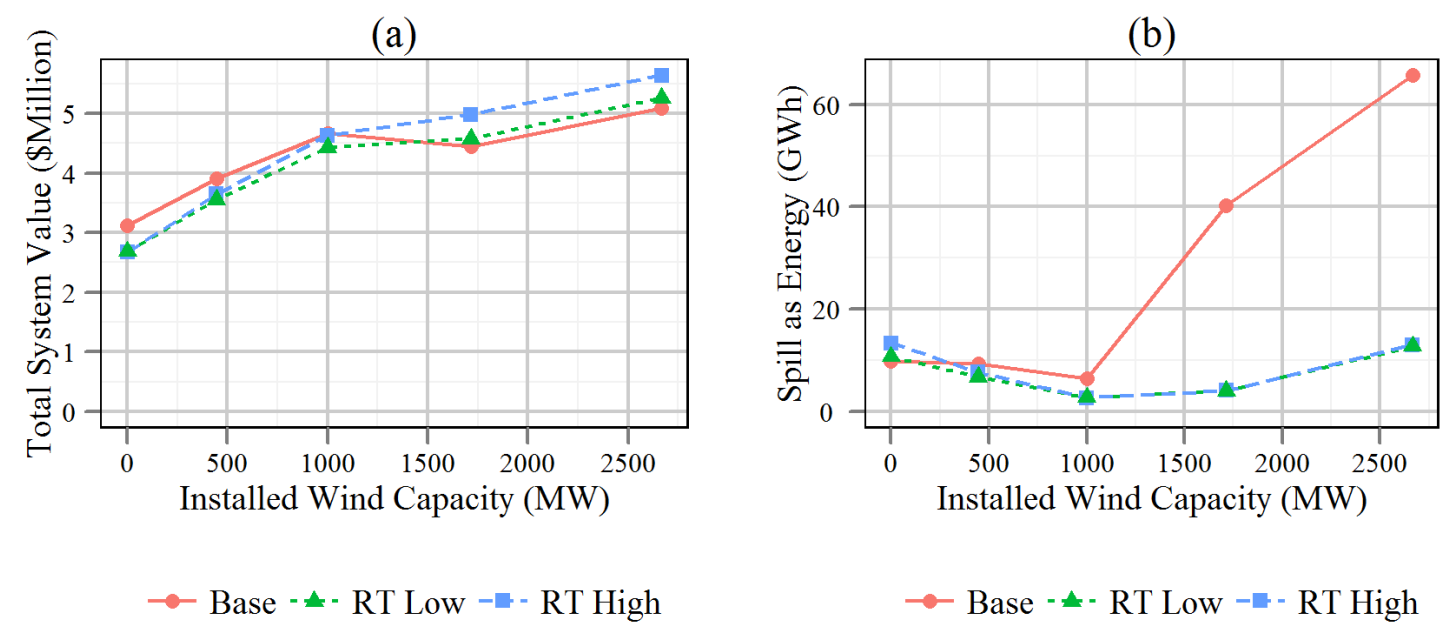

Figure 4.9: Real-time market impacts on (a) Total system value and (b) Spill as energy. RT Low represents a $10 \%$ decrease in RT MCP for each additional $50 \mathrm{MWh}$ of energy. RT High represents a $5 \%$ decrease for each $50 \mathrm{MWh}$.

The real-time scenarios actually show a reduction in total system value for the No Wind and $10 \%$ Wind cases. This is because the day-ahead and real-time markets are not being co-optimized. The objective to maximize the value of real-time energy in the operations run tends to leave the system with less water and thus less available capacity to sell as energy in the day-ahead market.

Assumptions about the load following reserve requirement are also tested by simply reducing the requirement. The net load variability and net load forecast error components are set to exceedence probabilities of 0.98 and 0.99 . (The Base case exceedence probability is 0.995 .) This can be assumed to be somewhat equivalent to assuming an improved wind forecast and thus a reduction in the amount of load following reserve required to cover potential forecast errors. The results 
shown in Figure 4.10 reveal little change at low wind penetrations but a significant impact at $30 \%$ penetration, increasing total system value by $13 \%$ and increasing the average value of added wind per unit of installed wind by $37 \%$ when using an exceedence probability of 0.98 . This comes at the expense, however, of an increase in the number of unmet load violations from 5 in the base $30 \%$ case to 10 in the LF0.99 case and 15 in the LF0.98 case. With the reduced load following reserve requirement the system is committing more capacity in the day-ahead market. This results in higher revenue, but at hours when significantly less wind arrives than was forecasted it no longer has enough reserve capacity to cover the full forecast error. These represent periods where it may be necessary to purchase energy at high prices to prevent loss of load events. In the model, unmet load is penalized at the initial MCP, representing a purchase cost for this energy. The sensitivity of the results to this penalty value is not evaluated here. The point of the analysis here is solely to test the sensitivity of the results to assumptions about the amount of scheduled reserves. The analysis indicates that at high wind penetration, the results are indeed quite sensitive to how the amount of required reserve capacity is determined and when the reserves and generation are scheduled. There is no difference in value at $40 \%$ wind across the three cases. This is again because the load following requirement is being violated to meet the spill limit constraint, so the system behaves the same even when the reserve requirement is higher.

A summary of sensitivities to variations in other system characteristics is provided here. For more detail on the analysis of these sensitivities refer to Appendix C. The value of added wind is sensitive to the level of the local load (see Figure C.15 in Appendix C). The wind generation adds greater value to the system in High Load scenarios. When more energy is being used to meet the local load and less is being committed in the market, the energy sold in the market has a higher marginal price. In the Low Load scenarios when there is already an excess of energy in the system, any additional energy sold into the market is at a lower marginal price. When variations in the transmission constraint are added to the different load scenarios (Figure C.16), the only benefit from added transmission is observed in the Low Load cases. In these cases, increasing the transmission limit adds value to the system by allowing additional excess energy to be bid into 
(a)

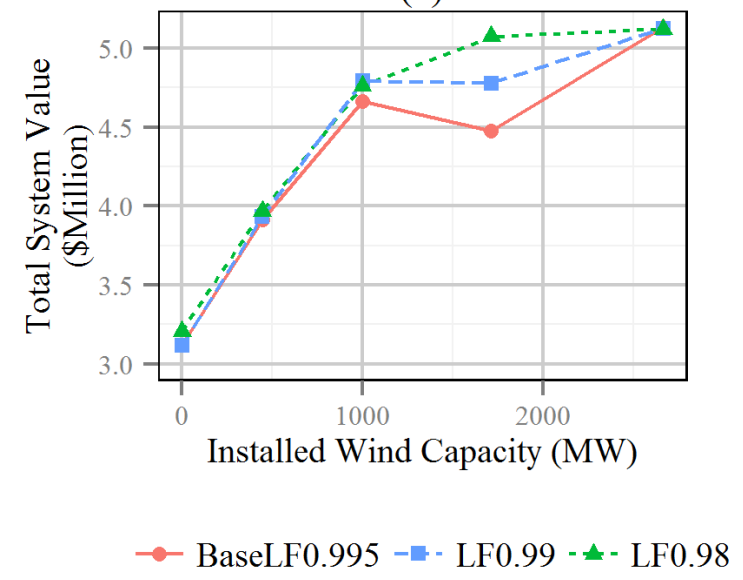

(b)

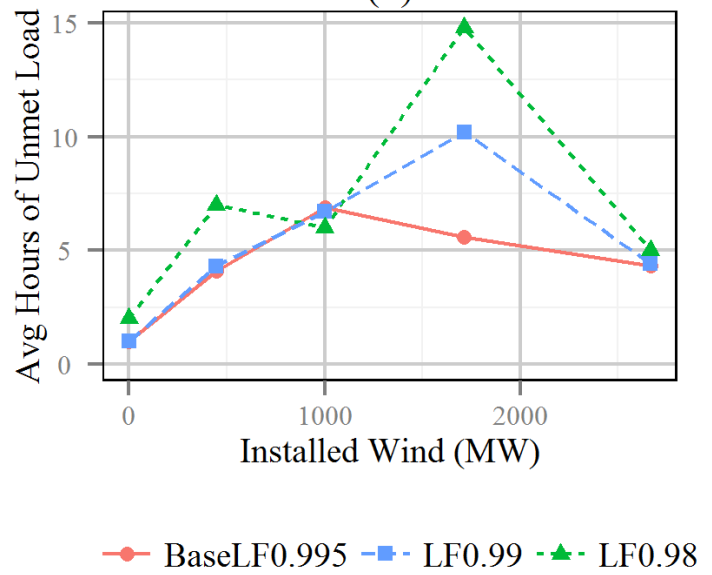

Figure 4.10: Comparison of different load following requirements for spring scenario on (a) Total system value and (b) Hours of unmet load (Note that in (a) the y-axis does not begin at zero.)

the market. Increased transmission capacity has no added value for the Base cases. Sensitivity to market depth is tested by adjusting the amount by which the day-ahead energy market clearing price decreases as more energy is sold into the market. While this does change the total system value due to the change in prices, the only change in system behavior is a slight shift in capacity away from day-ahead energy into ancillary services for the case with reduced depth in the energy market and the opposite shift when unlimited market depth is assumed (see Figure C.17).

The effects of integrated wind and hydropower can also be sensitive to the timing of the wind generation, whether the wind tends to be generally in phase or out of phase with load and prices. The wind in this system is generally in phase with load. This may not be the case for all geographic regions. In the No Wind case, there is a strong correlation, 0.79, between hydro generation and day-ahead energy prices. At $30 \%$ wind penetration, this correlation is reduced to only 0.23 because the added wind generation tends to displace a large portion of the hydropower generation during peak hours. The effects of wind timing are tested by running the Base scenario with the wind and associated forecasts shifted back 6,12 and 18 hours. All other inputs remain the same. When 
the wind is shifted, total system value decreases as the wind generation does not tend to coincide as well with peak pricing (see Figure C.18 in Appendix C). Effects on the correlation of hydro generation with energy prices are shown in Table 4.3. With a six hour shift, the correlation of hydro generation with prices does not decrease as much as in the Base case. A correlation of 0.42 at $30 \%$ is higher than the $30 \%$ Base case but still much lower than the No Wind case. Correlations in the 12-hour Shift case are similar to the Base case, while the 18-hour Shift case reduces the hydro generation and price correlation even more than the Base case.

Table 4.3: Correlation of hydro generation with day-ahead energy prices for various wind timing cases

\begin{tabular}{rrrrr}
\hline & Base & 6-hr Shift & 12-hr Shift & 18-hr Shift \\
\hline No Wind & 0.787 & 0.787 & 0.787 & 0.787 \\
$10 \%$ & 0.814 & 0.827 & 0.825 & 0.798 \\
$20 \%$ & 0.636 & 0.723 & 0.662 & 0.642 \\
$30 \%$ & 0.225 & 0.422 & 0.226 & 0.038 \\
$40 \%$ & 0.122 & 0.414 & 0.149 & -0.102 \\
\hline
\end{tabular}

\subsection{Conclusions}

A methodology has been developed to model integrated hydropower and wind generation using RiverWare modeling software that captures the effects on operations and economic value from both varied physical conditions and non-power policy. The methodology has been applied to a realistic test case over a range of scenarios to observe sensitivities to changes in physical inputs, system characteristics and modeling assumptions. Results indicate that the value of integrated wind and hydropower can be significantly impacted by the inclusion of non-power policy constraints, and a failure to account for such policy could lead to a misrepresentation of both the operational capacity for integrating wind and the economic value of the integrated system, particularly at high wind penetrations. The relative impact of non-power constraints, however, varies widely depending on wind and hydrologic conditions and the specifics of the policy. This suggests that it is difficult to generalize the impacts of non-power policy without running specific scenarios with actual policy 
constraints in place.

The integrated system does not have a linear response to changes in wind penetration. The interactions between the physical hydropower system and policy constraints can be quite complex. Model results, in general, are not proportional to changes in a single parameter. Rather sensitivities in the system are observed for combinations of factors together. This reinforces the importance of realistic modeling of the hydropower system. Without fully representing the complete, integrated system, the effect of these complex interactions on how the system responds to changing conditions may be overlooked.

Modeling results are sensitive to assumptions about the scheduling of generation and reserves in the hydropower system, namely that schedules are based on day-ahead forecasts. Altering these assumptions or including real-time market opportunities can increase the value of integration significantly. The benefits shown by real-time market participation are consistent with previous wind integration research.

Suggested extensions for this research include applying this methodology for a wider range of scenarios to further evaluate sensitivities in the system and modeling assumptions. In addition applying this approach for hydropower systems with different physical configurations, namely a hydro system with large storage reservoirs and pumped storage, would provide additional insight into the benefits and limitations for integrating wind and hydropower. This methodology could be incorporated into larger wind integration studies to provide an improved representation of hydropower and its role in balancing integrated wind generation. 


\section{Chapter 5}

\section{Conclusions and Recommendations}

\subsection{Summary}

A methodology has been developed for modeling integrated hydropower and wind generation that not only provides a representation of the physical characteristics of the hydropower system but also accounts for realistic non-power policy and how it limits hydropower's flexibility for balancing variable and uncertain wind generation. The methodology utilizes the optimization solver in RiverWare to carry out a two-stage optimization, a one week scheduling run followed by a one day operations run. This captures effects of adjusting the hydropower operations in real-time to balance deviations in the realized wind generation from the forecast. The methodology also incorporates the modeling of ancillary services into the framework. This is a significant component because one of the primary impacts of high levels of wind generation is the increased need for ancillary services that it places on the wider power system. The model captures how using hydropower to provide those services affects system operations.

In addition to providing a realistic representation of the physical hydropower system and its operating policy, the methodology also models the economic value of the integrated wind and hydropower system. This valuation includes energy, both day-ahead and real-time, sold into a power market as well as the economic value of ancillary services provided by the hydropower system. Rather than calculate an integration cost, the total value of the integrated system is calculated at different wind penetrations to determine the net value of wind added to the system. The economic evaluation also accounts for the impact that bidding additional energy and ancillary services into 
the market has on reducing market prices and thus approximates the effects that wind generation forecasts can have on market prices.

A wind generation forecast model was developed that can be applied to a known actual wind generation time series to produce forecast traces for day ahead, two day ahead and three day ahead forecasts. The forecast model uses an ARMA model fitted to observed wind generation forecast error from sample wind farms in order to capture statistical characteristics of the forecast error. The model captures the lag-1 and lag-2 autocorrelations well but tends to underestimate the variance in the forecast error.

The modeling methodology was applied to a test case integrated wind and hydropower system which includes five hydropower plants in a run-of-river configuration and wind penetrations ranging from $10 \%$ to $40 \%$. The model was run for scenarios with a range of hydrologic and wind conditions as well as with varying levels of policy constraints. In addition a sensitivity analysis was carried out on select system characteristics and model parameters to determine their impacts on model results.

\subsection{Conclusions}

Model results indicate that wind generation generally adds economic value to the integrated system at low penetrations. Also, at low penetrations, the hydropower system can handle the balancing requirements of the added wind generation without excessive difficulty. As the installed wind capacity increases, additional wind generation has diminishing returns, and at high enough penetrations, additional wind actually has a negative value for the system. As penetration increases, the added wind also causes greater operational difficulties for the system. One manner in which this observed is in an increase in the number of policy constraint violations for higher wind penetrations. The specific penetration level at which the added wind generation begins to cause operational difficulties and decrease in value depends on hydrologic and wind conditions. Results suggest, however, that in general it is between $20 \%$ and $30 \%$ wind penetration that either the total system value decreases or constraint violations due to wind begin to occur. This is consistent with previous 
hydropower and wind integration research. Previous studies have suggested that installed wind capacity less than approximately $20 \%$ can be handled by hydropower's balancing capabilities, but above $20 \%$ significant operational difficulties are expected to occur (see, for example, Acker et al., $2012 \mathrm{~b})$. This is not to say that installed wind capacity above $20 \%$ is not feasible or even potentially beneficial. High penetrations may, however, require additional economic consideration and modified operational procedures in order to benefit the power system.

The modeling results show that the effects of non-power policy constraints on integrated hydropower and wind generation are dependent on specific scenario conditions. The physical system and policy constraints interact with one another in a complex manner. The need to satisfy high priority environmental constraints can at times result in improved economic performance, but this comes at the expense of violating other system operational constraints. Utilization of goal programming makes it possible to capture the effects of these complex interactions. The response of the integrated system to changes in wind penetration or other individual parameters is nonlinear. This non-linear response and the complex interactions that produce this response emphasize the need for realistic hydropower modeling. Without complete modeling of both the physical interactions throughout the integrated hydropower system and its policy constraints, many of these effects would be missed.

The response of the integrated system to three different hydrologic and wind scenarios was investigated in this work. The spring scenario modeled relatively average stream flows. Nonpower constraints in place included elevated minimum environmental flow requirements at the final reservoir as well as limits on the within-the-day flow fluctuations at the same reservoir. In addition, minimum spill requirements for fish passage were in place for three reservoirs and maximum spill limits for TDG formation at all reservoirs. In this scenario, non-power constraints significantly reduced total system economic value. Their impacts on the effective system flexibility for providing reserves combined with the additional reserve requirements due to added wind generation resulted in a reduction of the capacity available to generate energy to sell into the market at high wind penetrations. This resulted in a negative net value for added wind at high (30\%) penetrations. 
The negative net value suggests that there may be an economic limit to the amount of added wind generation that is beneficial to the system that is different than the technical limit. Where this limit is, however, is dependent on a number of factors and modeling assumptions that have only been explored in part in this work. An increase in value at $40 \%$ in this scenario was one example where satisfying a high priority constraint (a maximum spill limit) improved the economic objective by violating a medium level reserve requirement. Occasional violations of the minimum flow requirement were observed in the spring scenario beginning at $10 \%$ wind and increasing at $20 \%$ and $30 \%$.

The summer scenario represented high flow and high wind conditions. This is often one of the critical operating conditions for hydropower systems with integrated wind generation. Minimum spill and minimum flow constraints were in place, but these had no impact on results because the system was already forced to pass excess water due to the high flow conditions. The controlling constraint for this scenario was the maximum spill limit for TDGs. Satisfaction of this higher priority spill constraint again resulted in more capacity being used for generation at the expense of violating reserve requirements. In the summer scenario this resulted in the Full Policy case returning a higher total system value than the reduced policy cases where the spill limit was not in effect. These situations in which the reserve requirements are violated to meet higher priority constraints represent periods when it might be necessary to either secure additional reserves from outside the system or curtail wind generation.

The fall scenario had the lowest flows and the least wind generation. The primary non-power constraint in this scenario limited outflows at the final reservoir to a narrow range during daylight hours. There were no minimum spill requirements. The non-power constraints in this this scenario had a much less noticeable effect on the system, reducing total system value only a small amount at the highest penetration levels. In this specific scenario wind generation continued to add value to the system at high penetrations.

Inclusion of non-power constraints in the spring scenario was observed to increase the variance in total system value across multiple wind forecast traces. This is illustrated in Figure C.1 in 
Appendix C. This same increase in variance was not observed for the summer and fall scenarios. Results with a wide variance indicate that running a single trace of forecast error is not sufficient for establishing a representative system response with a given set of policy constraints. Often constraints have a complex interaction with the physical system. Flow restrictions at one reservoir may impact operations and limit flexibility throughout the system. (An example of this is illustrated in Figure C.4 in Appendix C.) Modeling of the actual constraints within specific scenarios is required in order to assess their expected influence on system response to integrated wind generation.

A sensitivity analysis revealed that the results are highly influenced by assumptions about system scheduling and market structures. The assumption that the system is scheduled based on day-ahead forecasts and that schedule is not updated prior to operations results in a large load following requirement. At high levels of wind penetration (30\% in the spring scenario and $20 \%$ in the summer scenario), this high reserve requirement resulted in a decrease in total system value with added wind. Reducing the load following requirement allowed additional capacity to be used for generation at some time steps, increasing the total value for high wind cases, but this also resulted in more hours when the system could not meet the load. Adding participation in a realtime energy market represented the opportunity to schedule additional generation when a shorter forecast horizon is assumed. The shorter forecast horizon reduces the amount of reserve capacity required making it possible to utilize a portion of the scheduled reserve capacity for generation in the operations run. This also resulted in a substantial increase in system value at high wind penetrations but again increased the number of hours with unmet load by leaving the system with less water to use for generation at later times. Assumptions about how generation and reserves are scheduled would be specific to each hydropower system and would be dependent on the market structures available within each BA. Additional sensitivities that were evaluated are summarized in Appendix C. 


\section{$5.3 \quad$ Future Work}

The work presented here holds a number of opportunities for further research. One extension would be to apply the methodology to additional systems with different characteristics. Namely a hydropower system with large storage reservoirs is suggested. It would be insightful to analyze how the response of the run-of-river system modeled here compares to that of a system with significant storage. It would be helpful to assess how much additional flexibility the storage would add to the system. This would also provide opportunity to explore the impacts of different types of non-power policy. The operating objectives for a hydropower system with large storage would likely differ significantly from the run-of-river system modeled here. It may include operational objectives such as flood control and water delivery which were not considered in this model. Another valuable extension would be to add a pumped storage hydropower (PSH) facility into the model. PSH is expected to play a valuable role as a balancing reserve for high levels of renewable generation. Detailed analysis of a PSH facility within a realistic hydropower model would help quantify these potential benefits.

Another natural extension of the modeling framework presented here would be to apply it to a wider range of scenarios. One way to do this that would provide an added benefit would be to run the model for a longer time frame, such as a full year. The benefit of using one-week scenarios as we did here is that it allows for a detailed analysis of the sensitivities to changes in specific conditions is the system. One shortcoming of using one-week scenario runs is that effects from the week-ahead forecasts never get realized. It is not clear if the storage volumes at the end of the first week leave the system in a favorable position with sufficient flexibility to handle all possible realizations of conditions in the following week. Nor is it clearly evident how added wind generation affects this longer-term flexibility. An extended time horizon would allow for some of these longer term effects to be realized along with making it possible to model additional scenario conditions. From a functional standpoint, the current methodology would only require slight modifications to how data are imported into and exported from the model to make such extended runs feasible. 
Also, the current model results suggest that it would be beneficial to focus future work on wind penetration levels from $20 \%$ to $30 \%$. The system was generally able to handle wind generation at levels below $20 \%$ penetration without much difficulty. There tended to be a significant change in model outputs, however, when going from $20 \%$ to $30 \%$. Modeling intermediate wind levels between these two points would provide more complete information about critical transition points in system behavior and where they are located.

Sensitivities to additional modeling and policy assumptions could be explored further. For example, results from early provisional runs suggested that the tolerances which set how closely reservoir storage levels must meet target storages can have a significant effect on how the system responds to changing forecasts. This sensitivity was not evaluated systematically, however, with the final version of the model or with final input data. Similar sensitivities, such the tolerances for meeting scheduled generation and reserves in the operations run could be explored as well.

The wind forecast model could be improved to better capture the statistical characteristics of the wind forecast error. Specifically, an improved model could better capture the negative correlation of forecast error with actual wind generation. A possible approach would be to use a regression with correlated errors, though the results of using such a model have not been evaluated here. An improved methodology for producing synthetic wind forecast traces would be of benefit for the wider research topic of wind generation and its integration into the power grid beyond the specific application of hydropower and wind integration modeling.

As mentioned previously, the modeling results are sensitive to assumptions about system scheduling and its resulting impact on reserve requirements, primarily load following reserves in this model. These effects should be explored further. This could be done in several manners, and the specific approach would depend on the operational procedure of the actual system being modeled. One approach would be to use a wind forecast with reduced average error representing a forecast with shorter lead time and approximating a shorter scheduling horizon. This would tend to reduce the amount of reserve capacity that must be maintained in the system. Another approach would be to use the model to estimate the appropriate amount of reserves to schedule. 
The model could be run over a range of scenarios using various parameter values in the setting of load following requirements. Reliability metrics such as occurrences of unmet load could then be used to determine the appropriate load following requirement for the system. A final suggested approach that would require modification to the current modeling framework would be to include an intermediate run between the scheduling run and operations run with an updated forecast, presumably with less error, that would allow the system to be re-scheduled and would relax the load following reserve requirement due to the improved forecast. An additional point about scheduling assumptions in regards to actual operations of hydropower systems should also be made. This research suggests that in hydropower systems with large amounts of integrated wind generation, it may be beneficial or even necessary to modify scheduling procedures. Methodologies for system planning that were employed previously may no longer be sufficient in the context of increased variability and uncertainty. System operators may need to look to strategies such as updating schedules more frequently based on updated forecasts in order to meet the increasing demand on the hydropower system to provide flexible generation.

One final extension of this research would be to incorporate the hydropower modeling methodology into a larger wind integration study. This would address the original motivation behind this work and recommendations from previous large-scale wind integration studies such as the WWSIS (GE Energy, 2010) and the IEA Task 24 Final Report (Acker, 2011a,b) that hydropower modeling be improved in future wind integration studies. While the methodology presented here provides realistic modeling of the hydropower system, it does not model other generating resources directly. Combining the realistic hydropower model with a unit commitment model for the larger power system would provide a more complete representation of the response of the entire power system, including thermal generation sources and transmission, to integrated wind generation. Such model integration would be a work of much larger scope than the previously mentioned future work. It would present more technical challenges and would likely require collaboration from multiple parties with different modeling specialties. This type of collaboration and advancement in model integration will likely become more important moving forward, however, as the feasibility and benefits of 
integrating larger amounts of renewable generation into the power grid are explored. 


\section{Bibliography}

(2004), Hanford Reach Fall Chinook Protection Program Executed Agreement, available at http://www.nwd-wc.usace.army.mil/tmt/documents/wmp/2006/draft/app7.pdf.

Acker, T. (2011a), IEA Wind Task 24 Integration of Wind and Hydropower Systems, Volume 1: Issues, Impacts and Economcis of Wind and Hydropower Integration, Tech. Rep. NREL/TP5000-50181, National Renewable Energy Laboratory, Golden, Colorado.

Acker, T. (2011b), IEA Wind Task 24 Integration of Wind and Hydropower Systems, Volume 2: Participant Case Studies, Tech. Rep. NREL/TP-5000-50182, National Renewable Energy Laboratory, Golden, Colorado.

Acker, T., and C. Pete (2012), Western Wind and Solar Integration Study : Hydropower Analysis, Tech. Rep. NREL/SR-5500-53098, National Renewable Energy Laboratory, Golden, Colorado.

Acker, T. L., J. T. Buechler, K. Knitter, K. J. Conway, and R. Noteboom (2012a), Wind Integration Impacts on Hydropower Grant County PUD, Wind Engineering, 36(1), 81-96.

Acker, T. L., A. Robitaille, H. Holttinen, M. Piekutowski, and J. O. G. v. Tande (2012b), Integration of Wind and Hydropower Systems : Results of IEA Wind Task 24, Wind Engineering, 36(1), $1-18$.

Angarita, J. L., J. Usaola, and J. Martínez-Crespo (2009), Combined hydro-wind generation bids in a pool-based electricity market, Electric Power Systems Research, 79, 1038-1046, doi: 10.1016/j.epsr.2009.01.002.

Angarita, J. M., and J. G. Usaola (2007), Combining hydro-generation and wind energy biddings and operation on electricity spot markets, Electric Power Systems Research, 77, 393-400, doi: 10.1016/j.epsr.2006.03.019.

Belanger, C., and L. Gagnon (2002), Adding wind energy to hydropower, Energy Policy, 30, 12791284, doi:10.1016/S0140-6701(03)83022-9.

Benitez, L. E., P. C. Benitez, and G. C. van Kooten (2008), The economics of wind power with energy storage, Energy Economics, 30(4), 1973-1989, doi:10.1016/j.eneco.2007.01.017.

Black, M., and G. Strbac (2006), Value of storage in providing balancing services for electricity generation systems with high wind penetration, Journal of Power Sources, 162, 949-953, doi: 10.1016/j.jpowsour.2005.07.020. 
Bonneville Power Administration, U.S. Bureau of Reclamation, and U.S. Army Corps of Engineers (2009), 2010 Water Management Plan, available at http://www.nwdwc.usace.army.mil/tmt/documents/wmp/2010/final/app4.pdf.

Bueno, C., and J. A. Carta (2006), Wind powered pumped hydro storage systems, a means of increasing the penetration of renewable energy in the Canary Islands, Renewable and Sustainable Energy Reviews, 10(4), 312-340, doi:10.1016/j.rser.2004.09.005.

CADSWES (2012), RiverWare Documentation, available at http://cadswes.colorado.edu/PDF/RiverWare/documentation/.

de Almeida, A. T., P. S. Moura, A. S. Marques, and J. L. de Almeida (2005), Multi-impact evaluation of new medium and large hydropower plants in Portugal centre region, Renewable and Sustainable Energy Reviews, 9(2), 149-167, doi:10.1016/j.rser.2004.01.015.

de Mello, P. E., N. Lu, and Y. Makarov (2011), An optimized autoregressive forecast error generator for wind and load uncertainty study, Wind Energy, 14(February), 967-976, doi:10.1002/we.

DeCesaro, J., and K. Porter (2009), Wind Energy and Power System Operations : A Review of Wind Integration Studies to Date, Tech. Rep. NREL/SR-550-47256, National Renewable Energy Laboratory, Golden, Colorado.

Ela, E., M. Milligan, E. Lannoye, D. Flynn, M. O'Malley, and B. Zavadil (2010), Evolution of Operating Reserve Determination in Wind Power Integration Studies, in 2010 IEEE Power 8 Energy Society General Meeting, March 2011, National Renewable Energy Laboratory, Minneapolis, Minnesota.

Ela, E., M. Milligan, and B. Kirby (2011), Operating Reserves and Variable Generation Operating Reserves and Variable Generation, Tech. Rep. NREL/TP-5500-51978, National Renewable Energy Laboratory, Golden, Colorado.

Electric Power Research Institute (2011), MISO Energy Storage Study Phase 1 Report, Tech. Rep. 1024489, Electric Power Research Institute, Palo Alto, California.

EnerNex (2010), Eastern Wind Integration and Transmission Study, Tech. Rep. NREL/SR-550047078, Enernex Corporation, National Renewable Energy Laboratory, Golden, Colorado.

Eschenbach, E. A., T. Magee, E. Zagona, M. Goranflo, and R. Shane (2001), Goal Programming Decision Support System for Multiobjective Operation of Reservoir Systems, Journal of Water Resources Planning and Management, 127(2), 108-120, doi:10.1061/(ASCE)07339496(2001)127:2(108).

Eto, J. H., J. Undrill, P. Mackin, R. Daschmans, B. Williams, B. Haney, R. Hunt, J. Ellis, H. Illian, C. Martinez, M. O'Malley, K. Coughlin, and K. H. LaCommare (2010), Use of Frequency Response Metrics to Assess the Planning and Operating Requirements for Reliable Integration of Variable Renewable Generation, Tech. Rep. LBNL-4142E, Lawrence Berkeley National Laboratory, Berkeley, California.

GE Energy (2010), Western Wind and Solar Integration Study, Tech. Rep. NREL/SR-550-47434, GE Energy; National Renewable Energy Laboratory, Golden, Colorado. 
Hodge, B.-M., and M. Milligan (2011), Wind Power Forecasting Error Distributions over Multiple Timescales Preprint, Tech. Rep. NREL/CP-5500-50614, National Renewable Energy Laboratory, Golden, Colorado.

Hodge, B.-M., D. Lew, and M. Milligan (2011), The Impact of High Wind Power Penetrations on Hydroelectric Unit Operations in the WWSIS, Tech. Rep. NREL/TP-5500-52251, National Renewaable Energy Laboratory, Golden, Colorado.

Holttinen, H., and G. Koreneff (2012), Imbalance Costs of Wind Power for a Hydro Power Producer in Finland, Wind Engineering, 36(1), 53-68, doi:10.1260/0309-524X.36.1.53.

Holttinen, H., M. Milligan, B. Kirby, T. Acker, V. Neimane, and T. Molinski (2008), Using Standard Deviation as a Measure of Increased Operational Reserve Requirement for Wind Power, Wind Engineering, 32(4), 355-377, doi:10.1260/0309-524X.32.4.355.

Hug-Glanzmann, G. (2011a), A Hybrid Approach to Balance the Variability and Intermittency of Renewable Generation, in PowerTech, 2011 IEEE Trondheim, June, pp. 3-10, Trondheim, Norway.

Hug-Glanzmann, G. (2011b), Predictive Control for Balancing Wind Generation Variability using Run-of-River Power Plants, in IEEE Power Engineering Society General Meeting, 2011, pp. 1-8.

Jaramillo, O. A., M. A. Borja, and J. M. Huacuz (2004), Using hydropower to complement wind energy: a hybrid system to provide firm power, Renewable Energy, 29(11), 1887-1909, doi: 10.1016/j.renene.2004.02.010.

Jónsson, T., P. Pinson, and H. Madsen (2010), On the market impact of wind energy forecasts, Energy Economics, 32(2), 313-320, doi:10.1016/j.eneco.2009.10.018.

Kaldellis, J., M. Kapsali, and K. Kavadias (2010), Energy balance analysis of wind-based pumped hydro storage systems in remote island electrical networks, Applied Energy, 87(8), 2427-2437, doi:10.1016/j.apenergy.2010.02.016.

Karki, R., P. Hu, and R. Billinton (2010), Reliability assessment of a wind integrated hydro-thermal power system, in Probabilistic Methods Applied to Power Systems (PMAPS), 2010 IEEE 11th International Conference on, pp. 265-270, IEEE.

Klinge Jacobsen, H., and E. Zvingilaite (2010), Reducing the market impact of large shares of intermittent energy in Denmark, Energy Policy, 38(7), 3403-3413, doi:10.1016/j.enpol.2010.02.014.

Leite da Silva, A. M., M. A. Rosa, W. S. Sales, and M. Matos (2011), Long term evaluation of operating reserve with high penetration of renewable energy sources, in 2011 IEEE Power and Energy Society General Meeting, pp. 1-7, Ieee, doi:10.1109/PES.2011.6039333.

Loose, V. W. (2011), Quantifying the Value of Hydropower in the Electric Grid : Role of Hydropower in Existing Markets, Tech. Rep. SAND2011-1009, Sandia National Laboratories, Albuquerque, New Mexico.

Matevosyan, J., and L. Söder (2007), Short-term hydropower planning coordinated with wind power in areas with congestion problems, Wind Energy, 10(3), 195-208, doi:10.1002/we. 
Matevosyan, J., M. Olsson, and L. Soder (2009), Hydropower planning coordinated with wind power in areas with congestion problems for trading on the spot and the regulating market, Electric Power Systems Research, 79(1), 39-48, doi:10.1016/j.epsr.2008.05.019.

Milligan, M., and B. Kirby (2009), Calculating Wind Integration Costs : Separating Wind Energy Value from Integration Cost Impacts, Tech. Rep. NREL/TP-550-46275, National Renewable Energy Laboratory, Golden, Colorado.

Milligan, M., B. Kirby, R. Gramlich, and M. Goggin (2009), Impact of Electric Industry Structure on High Wind Penetration Potential Impact of Electric Industry Structure on High Wind Penetration Potential, Tech. Rep. NREL/TP-550-46273, National Renewable Energy Laboratory, Golden, Colorado.

Milligan, M., E. Ela, B.-M. Hodge, B. Kirby, D. Lew, C. Clark, J. DeCesaro, and K. Lynn (2011), Cost-Causation and Integration Cost Analysis for Variable Generation, Tech. Rep. NREL/TP5500-51860, National Renewable Energy Laboratory, Golden, Colorado.

Morales, J. M., A. J. Conejo, and J. Pérez-ruiz (2009), Economic Valuation of Reserves in Power Systems With High Penetration of Wind Power, IEEE Transactions on Power Systems, 24(2), 900-910.

North American Electric Reliability Corporation (2007), NERC Standard BAL-001-0a - Real Power Balancing Control Performance, available at http://www.nerc.com/files/BAL-001-0a.pdf.

Ortega-Vazquez, M. A., and D. S. Kirschen (2009), Estimating the Spinning Reserve Requirements in Systems With Significant Wind Power Generation Penetration, IEEE Transactions on Power Systems, 24(1), 114-124, doi:10.1109/TPWRS.2008.2004745.

Parsons, B., M. Milligan, J. Smith, E. DeMeo, B. Oakleaf, K. Wolf, M. Schuerger, R. Zavadil, M. Ahlstrom, and D. Nakafuji (2006), Grid Impacts of Wind Power Variability: Recent Assessments from a Variety of Utilities in the United States; Preprint, Tech. Rep. NREL/CP-500-39955, National Renewable Energy Laboratory (NREL), Golden, Colorado.

Piekutowski, M., A. Halley, and S. Denholm (2012), The Future Role of Hydro Plant in Maximising the Integration of Wind Generation The Tasmanian Case Study, Wind Engineering, 36(May 2005), 19-34.

Robitaille, A., I. Kamwa, A. H. Oussedik, M. de Montigny, N. Menemenlis, M. Huneault, A. Forcione, R. Mailhot, J. Bourret, and L. Bernier (2012), Preliminary Impacts of Wind Power Integration in the Hydro-Quebec System, Wind Engineering, 36(1), 35-52, doi:10.1260/0309524X.36.1.35.

Soder, L. (2004), Simulation of Wind Speed Forecast Errors for Operation Planning of MultiArea Power Systems, in 8th International Conference on Probabilistic Methods Applied to Power Systems, pp. 723-728, Ames, Iowa.

Taylor, J. W., and P. E. McSharry (2007), Short-Term Load Forecasting Methods : An Evaluation Based on European Data, IEEE Transactions on Power Systems, 22(4), 2213-2219.

Troy, N., E. Denny, and M. O'Malley (2010), Base-Load Cycling on a System With Significant Wind Penetration, IEEE Transactions on Power Systems, 25(2), 1088-1097, doi: 10.1109/TPWRS.2009.2037326. 
Tuohy, A., and M. O'Malley (2009), Impact of pumped storage on power systems with increasing wind penetration, in 2009 IEEE Power 85 Energy Society General Meeting, pp. 1-8, Ieee, doi: 10.1109/PES.2009.5275839.

Wan, Y. (2005), A Primer on Wind Power for Utility Applications A Primer on Wind Power for Utility Applications, Tech. Rep. NREL/TP-500-36230, National Renewable Energy Laboratory, Golden, Colorado.

Wan, Y.-h. (2004), Wind Power Plant Behaviors : Analyses of Long-Term Wind Power Data, Tech. Rep. NREL/TP-500-36551, National Renewable Energy Laboratory, Golden, Colorado.

Western Electricity Coordinating Council (2008), WECC Standard BAL-002-WECC-1 Contingency Reserves, available at http://www.nerc.com/files/BAL-002-WECC-1.pdf.

Zagona, E. A., T. J. Fulp, R. Shane, T. Magee, and H. M. Goranflo (2001), RiverWare: A generalized tool for complex reservoir system modeling, Journal of the American Water Resources Association, 37(4), 913-929.

Zima-Bockarjova, M., J. Matevosyan, M. Zima, and L. Soder (2010), Sharing of Profit From Coordinated Operation Planning and Bidding of Hydro and Wind Power, IEEE Transactions on Power Systems, 25(3), 1663-1673, doi:10.1109/TPWRS.2010.2040636. 
Appendix A

Plant Power Tables 
Table A.1: Plant power table for Reservoir 1B

\begin{tabular}{crr||crr}
\hline $\begin{array}{c}\text { Operating } \\
\text { Head }(\mathrm{ft})\end{array}$ & $\begin{array}{r}\text { Turbine } \\
\mathrm{Q}(\mathrm{kcfs})\end{array}$ & $\begin{array}{r}\text { Power } \\
(\mathrm{MW})\end{array}$ & $\begin{array}{c}\text { Operating } \\
\text { Head }(\mathrm{ft})\end{array}$ & $\begin{array}{r}\text { Turbine } \\
\mathrm{Q}(\mathrm{kcfs})\end{array}$ & $\begin{array}{r}\text { Power } \\
(\mathrm{MW})\end{array}$ \\
\hline 45 & 0.0 & 0.0 & 63 & 0.0 & 0.0 \\
45 & 115.0 & 393.8 & 63 & 119.5 & 573.7 \\
45 & 200.0 & 600.6 & 63 & 200.0 & 848.5 \\
47 & 0.0 & 0.0 & 65 & 0.0 & 0.0 \\
47 & 115.5 & 413.1 & 65 & 120.0 & 594.5 \\
47 & 200.0 & 627.9 & 65 & 200.0 & 876.3 \\
49 & 0.0 & 0.0 & 67 & 0.0 & 0.0 \\
49 & 116.0 & 432.6 & 67 & 120.5 & 615.5 \\
49 & 200.0 & 655.3 & 67 & 197.9 & 897.7 \\
51 & 0.0 & 0.0 & 69 & 0.0 & 0.0 \\
51 & 116.5 & 452.3 & 69 & 121.0 & 636.6 \\
51 & 200.0 & 682.8 & 69 & 195.7 & 918.5 \\
53 & 0.0 & 0.0 & 71 & 0.0 & 0.0 \\
53 & 117.0 & 472.2 & 71 & 121.5 & 657.8 \\
53 & 200.0 & 710.2 & 71 & 193.6 & 939.0 \\
55 & 0.0 & 0.0 & 73 & 0.0 & 0.0 \\
55 & 117.5 & 492.2 & 73 & 122.0 & 679.3 \\
55 & 200.0 & 737.8 & 73 & 191.4 & 959.0 \\
57 & 0.0 & 0.0 & 75 & 0.0 & 0.0 \\
57 & 118.0 & 512.3 & 75 & 122.5 & 700.8 \\
57 & 200.0 & 765.4 & 75 & 189.3 & 978.5 \\
59 & 0.0 & 0.0 & 77 & 0.0 & 0.0 \\
59 & 118.5 & 532.6 & 77 & 123.0 & 722.6 \\
59 & 200.0 & 793.1 & 77 & 187.1 & 997.6 \\
61 & 0.0 & 0.0 & 79 & 0.0 & 0.0 \\
61 & 119.0 & 553.1 & 79 & 123.5 & 744.5 \\
61 & 200.0 & 820.8 & 79 & 185.0 & 1016.3 \\
\hline \hline Operating & Head for Opt & $71(\mathrm{ft})$ & & & \\
\hline & & & & &
\end{tabular}


Table A.2: Plant power table for Reservoir 1C

\begin{tabular}{crr||crr}
\hline $\begin{array}{c}\text { Operating } \\
\text { Head }(\mathrm{ft})\end{array}$ & $\begin{array}{r}\text { Turbine } \\
\mathrm{Q}(\mathrm{kcfs})\end{array}$ & $\begin{array}{r}\text { Power } \\
(\mathrm{MW})\end{array}$ & $\begin{array}{c}\text { Operating } \\
\text { Head }(\mathrm{ft})\end{array}$ & $\begin{array}{r}\text { Turbine } \\
\mathrm{Q}(\mathrm{kcfs})\end{array}$ & $\begin{array}{r}\text { Power } \\
(\mathrm{MW})\end{array}$ \\
\hline 73 & 0.0 & 0.0 & 87 & 0.0 & 0.0 \\
73 & 115.0 & 646.7 & 87 & 120.0 & 803.5 \\
73 & 200.0 & 988.2 & 87 & 200.0 & 1185.9 \\
75 & 0.0 & 0.0 & 89 & 0.0 & 0.0 \\
75 & 115.7 & 668.5 & 89 & 120.7 & 826.7 \\
75 & 200.0 & 1016.2 & 89 & 197.5 & 1204.0 \\
77 & 0.0 & 0.0 & 91 & 0.0 & 0.0 \\
77 & 116.4 & 690.5 & 91 & 121.4 & 850.2 \\
77 & 200.0 & 1044.4 & 91 & 195.0 & 1221.6 \\
79 & 0.0 & 0.0 & 93 & 0.0 & 0.0 \\
79 & 117.1 & 712.6 & 93 & 122.1 & 873.9 \\
79 & 200.0 & 1072.6 & 93 & 192.5 & 1238.6 \\
81 & 0.0 & 0.0 & 95 & 0.0 & 0.0 \\
81 & 117.9 & 735.0 & 95 & 122.9 & 897.8 \\
81 & 200.0 & 1100.8 & 95 & 190.0 & 1255.0 \\
83 & 0.0 & 0.0 & 97 & 0.0 & 0.0 \\
83 & 118.6 & 757.6 & 97 & 123.6 & 921.9 \\
83 & 200.0 & 1129.1 & 97 & 187.5 & 1270.8 \\
85 & 0.0 & 0.0 & 99 & 0.0 & 0.0 \\
85 & 119.3 & 780.5 & 99 & 124.3 & 946.2 \\
85 & 200.0 & 1157.5 & 99 & 185.0 & 1286.0 \\
\hline \hline Operating & Head for Opt & $91(\mathrm{ft})$ & & & \\
\hline \multicolumn{5}{r}{} & \multicolumn{3}{c}{}
\end{tabular}


Table A.3: Plant power table for Reservoir 1D

\begin{tabular}{|c|c|c|c|c|c|}
\hline $\begin{array}{l}\text { Operating } \\
\text { Head (ft) }\end{array}$ & $\begin{array}{l}\text { Turbine } \\
\text { Q (kcfs) }\end{array}$ & $\begin{array}{l}\text { Power } \\
\text { (MW) }\end{array}$ & $\begin{array}{l}\text { Operating } \\
\text { Head (ft) }\end{array}$ & $\begin{array}{l}\text { Turbine } \\
\text { Q (kcfs) }\end{array}$ & $\begin{array}{l}\text { Power } \\
\text { (MW) }\end{array}$ \\
\hline 18 & 0.0 & 0.0 & 36 & 0.0 & 0.0 \\
\hline 18 & 115.0 & 155.6 & 36 & 119.5 & 323.9 \\
\hline 18 & 200.0 & 236.9 & 36 & 200.0 & 478.3 \\
\hline 20 & 0.0 & 0.0 & 38 & 0.0 & 0.0 \\
\hline 20 & 115.5 & 173.7 & 38 & 120.0 & 343.4 \\
\hline 20 & 200.0 & 263.5 & 38 & 200.0 & 505.4 \\
\hline 22 & 0.0 & 0.0 & 40 & 0.0 & 0.0 \\
\hline 22 & 116.0 & 191.9 & 40 & 120.5 & 363.0 \\
\hline 22 & 200.0 & 290.2 & 40 & 197.9 & 528.7 \\
\hline 24 & 0.0 & 0.0 & 42 & 0.0 & 0.0 \\
\hline 24 & 116.5 & 210.3 & 42 & 121.0 & 382.8 \\
\hline 24 & 200.0 & 316.9 & 42 & 195.7 & 551.6 \\
\hline 26 & 0.0 & 0.0 & 44 & 0.0 & 0.0 \\
\hline 26 & 117.0 & 228.8 & 44 & 121.5 & 402.8 \\
\hline 26 & 200.0 & 343.7 & 44 & 193.6 & 574.1 \\
\hline 28 & 0.0 & 0.0 & 46 & 0.0 & 0.0 \\
\hline 28 & 117.5 & 247.5 & 46 & 122.0 & 422.9 \\
\hline 28 & 200.0 & 370.5 & 46 & 191.4 & 596.2 \\
\hline 30 & 0.0 & 0.0 & 48 & 0.0 & 0.0 \\
\hline 30 & 118.0 & 266.4 & 48 & 122.5 & 443.2 \\
\hline 30 & 200.0 & 397.4 & 48 & 189.3 & 618.0 \\
\hline 32 & 0.0 & 0.0 & 50 & 0.0 & 0.0 \\
\hline 32 & 118.5 & 285.4 & 50 & 123.0 & 463.6 \\
\hline 32 & 200.0 & 424.3 & 50 & 187.1 & 639.3 \\
\hline 34 & 0.0 & 0.0 & 52 & 0.0 & 0.0 \\
\hline 34 & 119.0 & 304.6 & 52 & 123.5 & 484.2 \\
\hline 34 & 200.0 & 451.3 & 52 & 185.0 & 660.1 \\
\hline Operating & $\mathrm{d}$ for Opt & $36(\mathrm{ft})$ & & & \\
\hline
\end{tabular}


Table A.4: Plant power table for Reservoir 1E

\begin{tabular}{|c|c|c|c|c|c|}
\hline $\begin{array}{l}\text { Operating } \\
\text { Head (ft) }\end{array}$ & $\begin{array}{l}\text { Turbine } \\
\text { Q (kcfs) }\end{array}$ & $\begin{array}{l}\text { Power } \\
\text { (MW) }\end{array}$ & $\begin{array}{l}\text { Operating } \\
\text { Head }(\mathrm{ft})\end{array}$ & $\begin{array}{l}\text { Turbine } \\
\text { Q (kcfs) }\end{array}$ & $\begin{array}{l}\text { Power } \\
\text { (MW) }\end{array}$ \\
\hline 55 & 0.0 & 0.0 & 75 & 0.0 & 0.0 \\
\hline 55 & 105.0 & 437.5 & 75 & 108.8 & 619.9 \\
\hline 55 & 180.0 & 655.6 & 75 & 180.0 & 903.2 \\
\hline 57 & 0.0 & 0.0 & 77 & 0.0 & 0.0 \\
\hline 57 & 105.4 & 455.2 & 77 & 109.2 & 638.9 \\
\hline 57 & 180.0 & 680.2 & 77 & 180.0 & 928.2 \\
\hline 59 & 0.0 & 0.0 & 79 & 0.0 & 0.0 \\
\hline 59 & 105.8 & 473.0 & 79 & 109.6 & 658.0 \\
\hline 59 & 180.0 & 704.8 & 79 & 180.0 & 953.3 \\
\hline 61 & 0.0 & 0.0 & 81 & 0.0 & 0.0 \\
\hline 61 & 106.2 & 490.9 & 81 & 110.0 & 677.1 \\
\hline 61 & 180.0 & 729.4 & 81 & 180.0 & 978.4 \\
\hline 63 & 0.0 & 0.0 & 83 & 0.0 & 0.0 \\
\hline 63 & 106.5 & 509.0 & 83 & 110.4 & 696.5 \\
\hline 63 & 180.0 & 754.1 & 83 & 177.5 & 994.3 \\
\hline 65 & 0.0 & 0.0 & 85 & 0.0 & 0.0 \\
\hline 65 & 106.9 & 527.1 & 85 & 110.8 & 715.9 \\
\hline 65 & 180.0 & 778.8 & 85 & 175.0 & 1009.7 \\
\hline 67 & 0.0 & 0.0 & 87 & 0.0 & 0.0 \\
\hline 67 & 107.3 & 545.4 & 87 & 111.2 & 735.5 \\
\hline 67 & 180.0 & 803.6 & 87 & 172.5 & 1024.4 \\
\hline 69 & 0.0 & 0.0 & 89 & 0.0 & 0.0 \\
\hline 69 & 107.7 & 563.9 & 89 & 111.5 & 755.2 \\
\hline 69 & 180.0 & 828.4 & 89 & 170.0 & 1038.6 \\
\hline 71 & 0.0 & 0.0 & 91 & 0.0 & 0.0 \\
\hline 71 & 108.1 & 582.4 & 91 & 111.9 & 775.0 \\
\hline 71 & 180.0 & 853.3 & 91 & 167.5 & 1052.2 \\
\hline 73 & 0.0 & 0.0 & 93 & 0.0 & 0.0 \\
\hline 73 & 108.5 & 601.1 & 93 & 112.3 & 795.0 \\
\hline 73 & 180.0 & 878.2 & 93 & 165.0 & 1065.2 \\
\hline Operating & for Opt & $85(\mathrm{ft})$ & & & \\
\hline
\end{tabular}


Table A.5: Plant power table for Reservoir 1F

\begin{tabular}{crr||crr}
\hline $\begin{array}{c}\text { Operating } \\
\text { Head }(\mathrm{ft})\end{array}$ & $\begin{array}{r}\text { Turbine } \\
\mathrm{Q}(\mathrm{kcfs})\end{array}$ & $\begin{array}{r}\text { Power } \\
(\mathrm{MW})\end{array}$ & $\begin{array}{c}\text { Operating } \\
\text { Head }(\mathrm{ft})\end{array}$ & $\begin{array}{r}\text { Turbine } \\
\mathrm{Q}(\mathrm{kcfs})\end{array}$ & $\begin{array}{r}\text { Power } \\
(\mathrm{MW})\end{array}$ \\
\hline 64 & 0.0 & 0.0 & 78 & 0.0 & 0.0 \\
64 & 115.0 & 564.8 & 78 & 120.0 & 717.5 \\
64 & 200.0 & 862.4 & 78 & 200.0 & 1058.5 \\
66 & 0.0 & 0.0 & 80 & 0.0 & 0.0 \\
66 & 115.7 & 586.0 & 80 & 120.7 & 740.2 \\
66 & 200.0 & 890.3 & 80 & 197.5 & 1077.5 \\
68 & 0.0 & 0.0 & 82 & 0.0 & 0.0 \\
68 & 116.4 & 607.3 & 82 & 121.4 & 763.1 \\
68 & 200.0 & 918.2 & 82 & 195.0 & 1095.9 \\
70 & 0.0 & 0.0 & 84 & 0.0 & 0.0 \\
70 & 117.1 & 629.0 & 84 & 122.1 & 786.2 \\
70 & 200.0 & 946.1 & 84 & 192.5 & 1113.8 \\
72 & 0.0 & 0.0 & 86 & 0.0 & 0.0 \\
72 & 117.9 & 650.8 & 86 & 122.9 & 809.5 \\
72 & 200.0 & 974.1 & 86 & 190.0 & 1131.1 \\
74 & 0.0 & 0.0 & 88 & 0.0 & 0.0 \\
74 & 118.6 & 672.8 & 88 & 123.6 & 833.0 \\
74 & 200.0 & 1002.2 & 88 & 187.5 & 1147.8 \\
76 & 0.0 & 0.0 & 90 & 0.0 & 0.0 \\
76 & 119.3 & 695.1 & 90 & 124.3 & 856.8 \\
76 & 200.0 & 1030.3 & 90 & 185.0 & 1164.0 \\
\hline \hline Operating & Head for Opt & $84(\mathrm{ft})$ & & & \\
\hline \multicolumn{5}{r}{} & \multicolumn{3}{c}{}
\end{tabular}




\section{Appendix B}

\section{Wind Modeling Supplemental Analysis}

\section{B.1 Wind Variability Analysis}

Table B.1 shows the site ID numbers for the selected sites used from the WWSIS 2006 dataset $^{1}$ used to scale the variability of wind at each penetration (see Section 3.1). Sites for each penetration include all of the sites from lower penetrations as well (i.e. the $40 \%$ case uses all sites in the table).

Table B.1: Site ID numbers for WWSIS sites used for wind variability analysis

\begin{tabular}{rr|rr|rr|rr}
\hline $10 \%$ & Sites & $20 \%$ & Sites & $30 \%$ & Sites & $40 \%$ & Sites \\
\hline 29026 & 28978 & 29027 & 28931 & 28731 & 27310 & 29025 & 28799 \\
29028 & & 29044 & 28932 & 28732 & 27312 & 29029 & 28800 \\
29059 & & 29045 & 29759 & 28733 & 27346 & 29043 & 28917 \\
28933 & & 29060 & 29766 & 28745 & 27347 & 29046 & 28977 \\
28934 & & 29061 & 29779 & 27846 & 27348 & 29084 & 28979 \\
28935 & & 29062 & 29789 & 28747 & 27349 & 29085 & 27475 \\
28947 & & 28898 & 27292 & 28762 & & 29086 & 27476 \\
28948 & & 28899 & 27293 & 28763 & & 29087 & 27477 \\
28949 & & 28900 & 27311 & 28764 & & 28789 & 27519 \\
28950 & & 28918 & & 28780 & & 28790 & 27520 \\
28963 & & 28919 & & 28781 & & 28791 & 27521 \\
28964 & & 28920 & & 28782 & & 28797 & 27559 \\
28965 & & 28921 & & 27291 & & 28798 & 27560 \\
\hline
\end{tabular}

WWSIS sites were selected so that the aggregated mean and the 10-minute coefficient of

\footnotetext{
1 The WWSIS dataset is provided by the National Renewable Energy Laboratory, which is operated by the Alliance for Sustainable Energy, LLC for the U.S. Department Of Energy and is available at http://www.nrel.gov/wind/integrationdatasets/western/data.html.
} 
variation $\left(C O V_{10}=\sigma_{10} / \mu\right)$ for the full 2006 dataset were approximately equal to the mean and 10-minute $C O V$ of the obeserved BPA wind generation data from 2010. This was considered to represent the $40 \%$ case. Mean, standard deviation and $C O V$ for the 10 -minute wind generation data from the seleceted WWSIS sites for the 2006 dataset along with the BPA 2010 data are shown in Table B.2.

Table B.2: 10-minute wind generation data statistics for one full year of BPA observed data from 2010 and penetration levels based on the 2006 scenario for selected WWSIS sites (see Table B.1)

\begin{tabular}{rrrrrr}
\hline & $10 \%$ & $20 \%$ & $30 \%$ & $40 \%$ & BPA \\
\hline Mean, $\mu$ (MW) & 135.65 & 296.91 & 484.90 & 796.77 & 787.46 \\
Std Dev, $\sigma_{10}(\mathrm{MW})$ & 150.50 & 317.21 & 511.75 & 833.29 & 824.57 \\
$C O V_{10}$ & 1.109 & 1.068 & 1.055 & 1.046 & 1.047 \\
\hline
\end{tabular}

Ten-minute wind data were averaged to hourly data, and again the mean, standard deviation and $C O V$ were calculated. The hourly standard deviation values shown in Table B.3 are the values used in equation 3.4 to scale the wind variability for each penetration level.

Table B.3: Hourly wind generation data statistics for selected WWSIS sites (2006) and BPA observed data (2010)

\begin{tabular}{rrrrrr}
\hline & $10 \%$ & $20 \%$ & $30 \%$ & $40 \%$ & BPA \\
\hline Mean, $\mu$ (MW) & 135.65 & 296.91 & 484.90 & 796.77 & 787.46 \\
Std Dev, $\sigma_{60}(\mathrm{MW})$ & 149.54 & 315.71 & 509.72 & 830.37 & 822.91 \\
$C O V_{60}$ & 1.102 & 1.063 & 1.051 & 1.042 & 1.045 \\
\hline
\end{tabular}

\section{B.2 Wind Forecast Model Analysis}

Analysis of the observed wind generation forecast error shows that there is a strong correlation between actual wind generation and wind generation forecast error. When the actual wind generation is low, the forecast tends to over-predict the wind, and thus the forecast error tends to be positive. When the actual wind is high, the opposite is true. The forecast tends to underpredict the wind, and the error is negative. Figure B.1 illustrates this. While there is a correlation between 
the forecast and the actual wind (0.4), there is a stronger correlation between the forecast error and actual wind (-0.7).

(a)

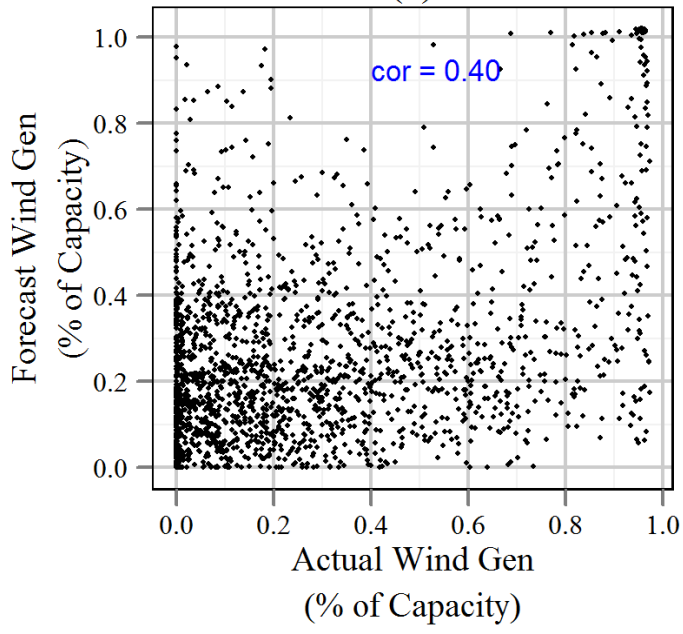

(b)

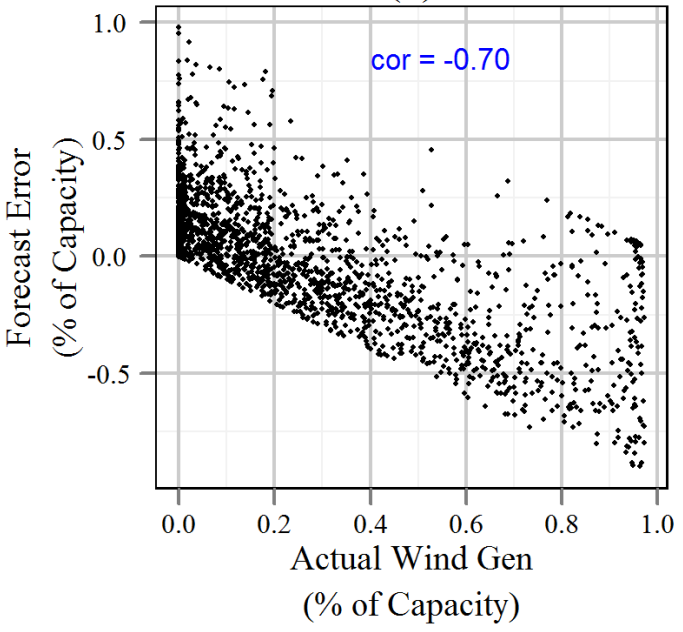

Figure B.1: Scatter plots of (a) Observed wind generation forecasts vs. actual wind generation and (b) Observed forecast error vs. actual wind generation

The dependency of wind forecast error on the actual generation can also be seen by examining the distribution of wind forecast error classified by the actual wind generation level. Figure B.2 divides the forecast error data by tercile of the corresponding actual wind generation. It is clear that the forecast error distributions change significanty with the actual wind generation with the lower regime error displaying a positive mean and positive skew and the upper regime error displaying a negative mean.

Figure B.2 also shows the distribution of the model forecast error, and it is clear that while the model does a decent job of capturing the distribution for the lower and mid regimes, it does not capture the distribution of the error for the upper regime as well. This is the primary reason that the model forecast error has less variance and a reduced skew when compared with the observed error.

An improved model would better capture the dependency of forecast error on the actual wind 


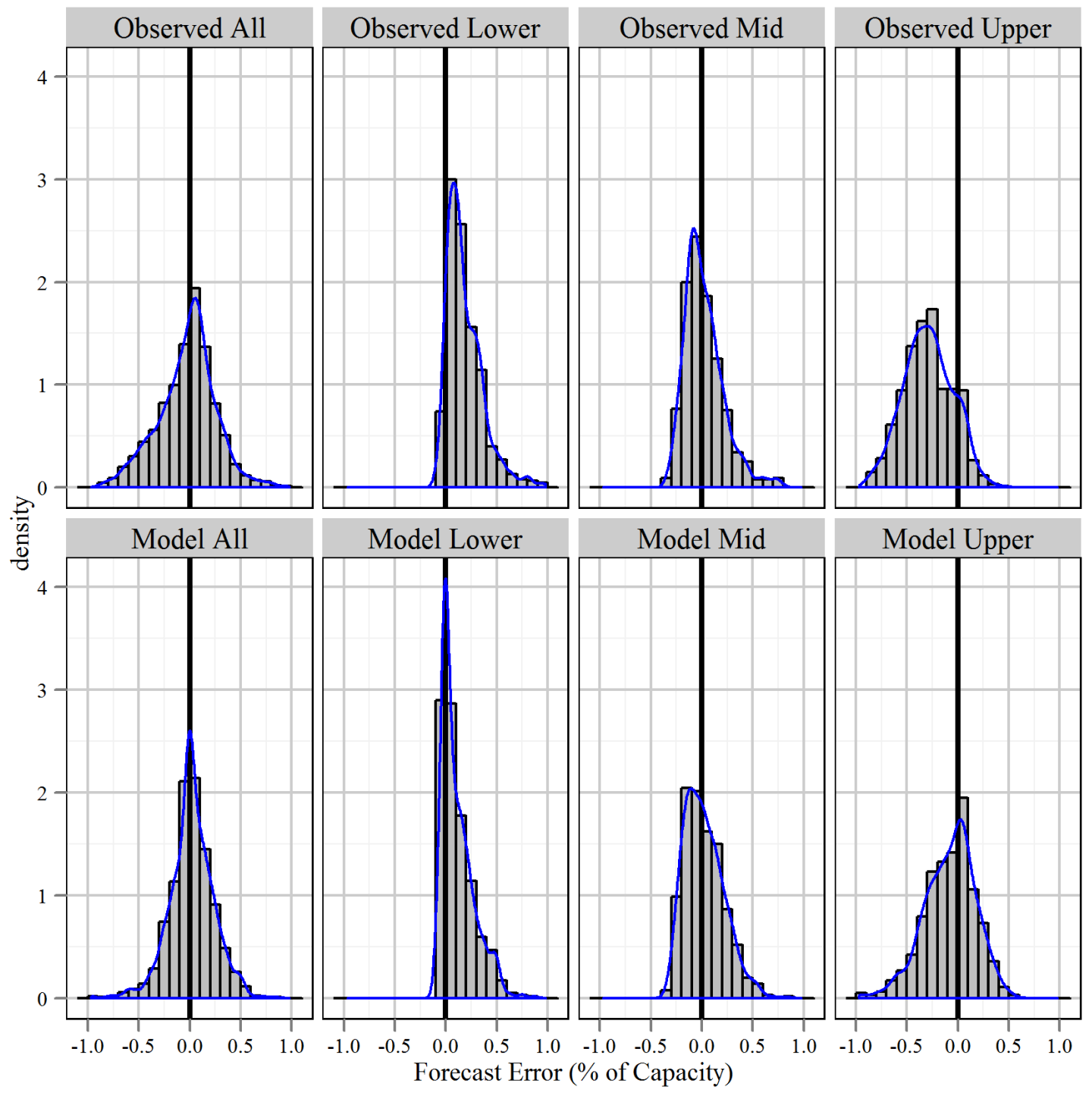

Figure B.2: Histograms of observed and modeled wind forecast percent error showing all data and classified by the actual wind generation level divided in terciles

generation. A suggested alternative would be to use a regression with correlated errors, though the results from using such a model are not evaluated here. 


\section{Appendix $\mathrm{C}$}

\section{Additional Model Results and Analysis}

\section{C.1 Policy Effects on System Economic Value}

The non-power policy not only affects the average system value with wind integration. It may also increase the variance in value across multiple traces. Figure C.1 shows that the variance in total system value for the ten forecast traces modeled in spring scenario is larger in the Base Full Policy case than the redued policy cases. Slight changes to inputs may have a larger effect on the system when it is more restricted leading to more variation in the response. This same effect is not observed as clearly for the summer and fall scenarios, however. The reduced policy scenarios tend to have a wider variance for these scenarios. Table C.1 shows the standard deviation of total system value for all three scenarios and all penetration levels.

A wide variance among forecast traces would suggest that it is not sufficient to run a single trace or even a small number of traces when attempting to characterize the expected response of the system in a given scenario. Running a single trace in the spring Full Policy case could result in a misrepresentation of the expected behavior of the system for those seasonal conditions. 

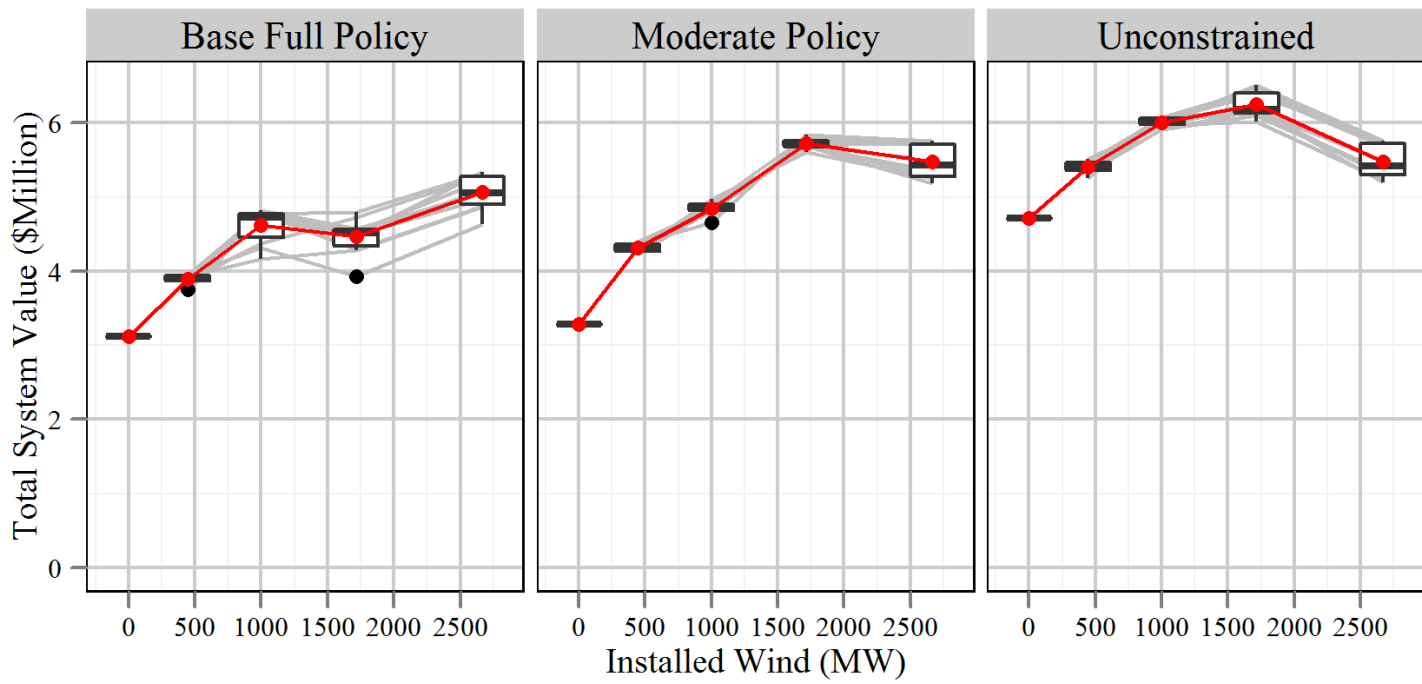

Figure C.1: Spring scenario variance in total system value for multiple wind forecast traces (gray lines) with full and reduced policy: Red points represent the mean values. Boxes correspond to the inter-quartile range (IQR, 25th to 75th percentiles). Black bars are at the median value. Whiskers extend to the largest and smallest values within $1.5^{*} \mathrm{IQR}$. Black points are outliers, outside of $1.5^{*} \mathrm{IQR}$.
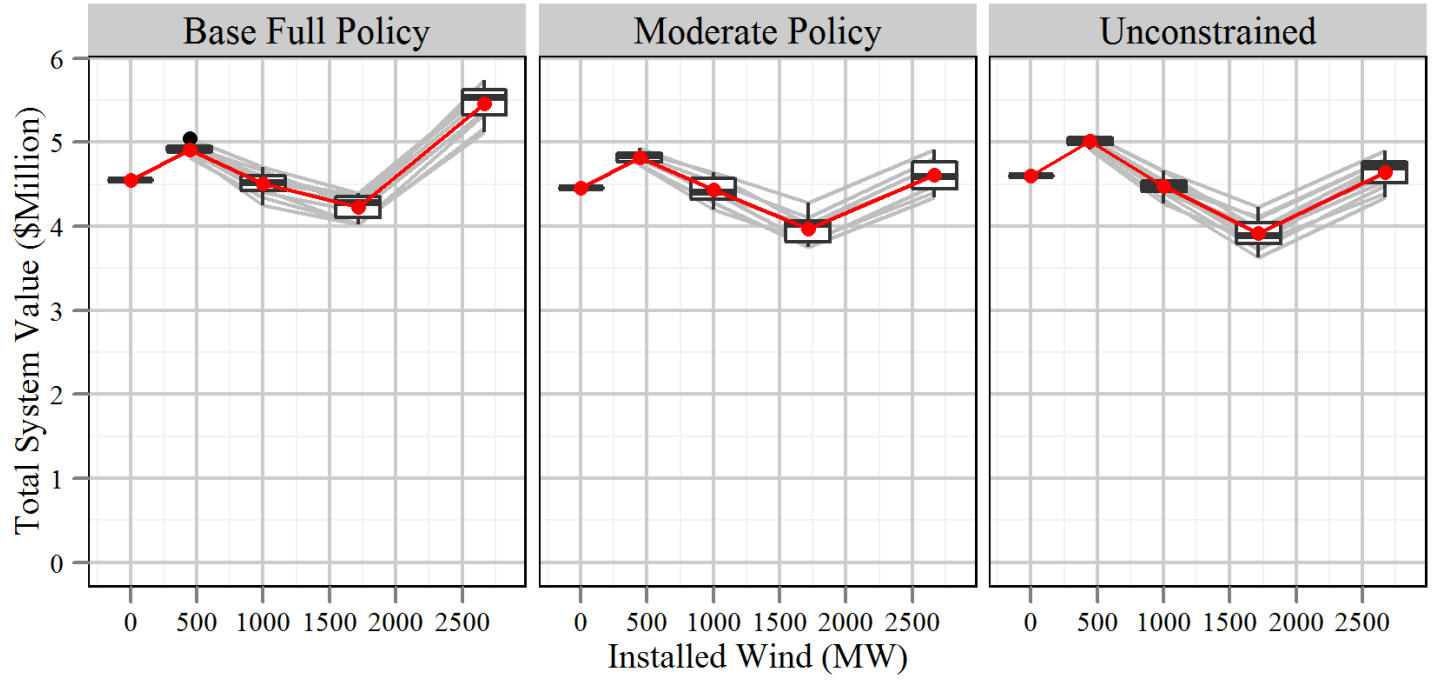

Figure C.2: Summer scenario variance in total system value for multiple wind forecast traces (gray lines) with full and reduced policy: Red points represent the mean values. Boxes correspond to the inter-quartile range (IQR, 25th to 75th percentiles). Black bars are at the median value. Whiskers extend to the largest and smallest values within $1.5^{*} \mathrm{IQR}$. Black points are outliers, outside of $1.5^{*} \mathrm{IQR}$. 

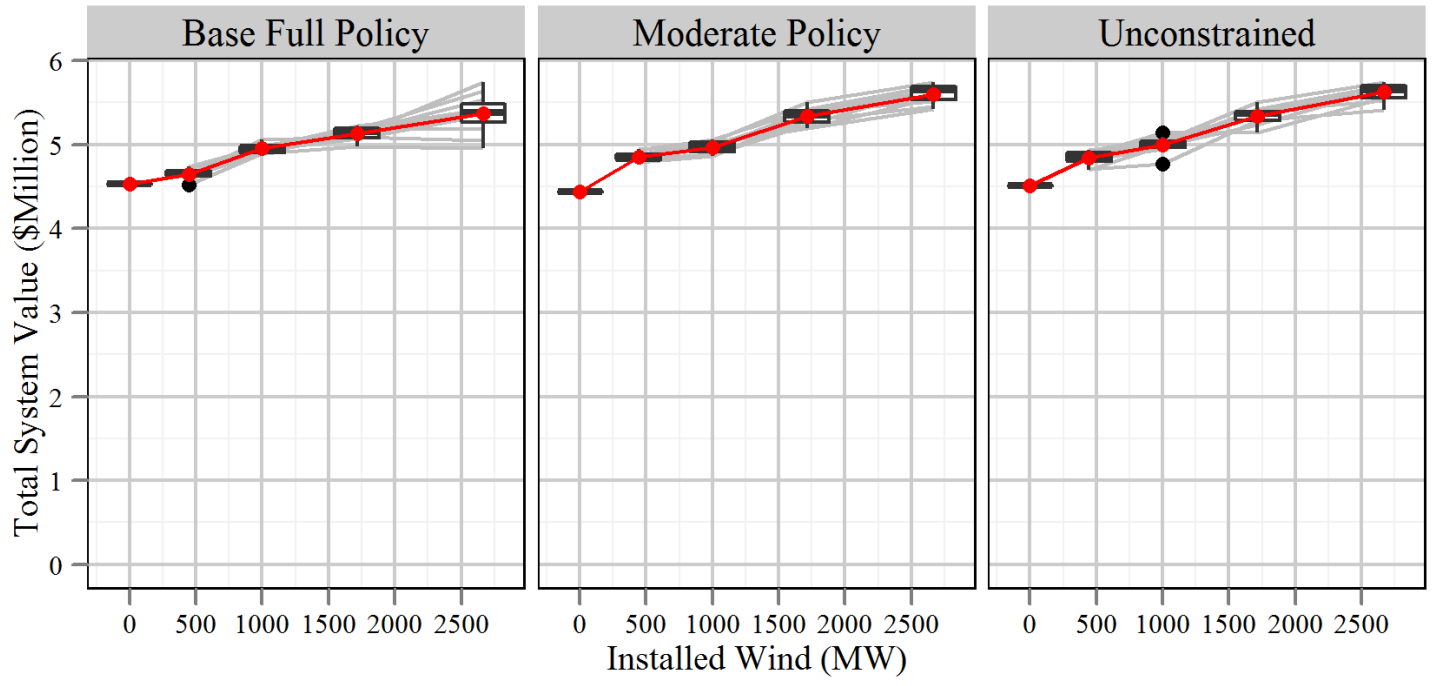

Figure C.3: Fall scenario variance in total system value for multiple wind forecast traces (gray lines) with full and reduced policy: Red points represent the mean values. Boxes correspond to the inter-quartile range (IQR, 25th to 75th percentiles). Black bars are at the median value. Whiskers extend to the largest and smallest values within $1.5^{*} \mathrm{IQR}$. Black points are outliers, outside of $1.5^{*} \mathrm{IQR}$.

Table C.1: Standard deviation (in dollars) of total system value across multiple traces for each hydrologic scenario

\begin{tabular}{rrrr}
\hline & Base Full Policy & Moderate Policy & Unsconstrained \\
\hline Spring 10\% & 78022 & 61564 & 78939 \\
Spring 20\% & 237532 & 84204 & 63990 \\
Spring 30\% & 249793 & 68730 & 169575 \\
Spring 40\% & 233484 & 227945 & 231597 \\
\hline Summer 10\% & 58935 & 75543 & 61192 \\
Summer 20\% & 136906 & 167012 & 121482 \\
Summer 30\% & 147013 & 187765 & 182315 \\
Summer 40\% & 208154 & 213254 & 179559 \\
\hline Fall 10\% & 64808 & 51859 & 88868 \\
Fall 20\% & 50889 & 67939 & 103983 \\
Fall 30\% & 73354 & 97652 & 107004 \\
Fall 40\% & 234144 & 109137 & 105823 \\
\hline
\end{tabular}




\section{C.2 Impacts of Flow Constraints on Upstream Reservoir Operations}

Flow restrictions at one reservoir can have an impact on operations and thus flexibility throughout the system. An example of this is illustrated in Figure C.4. The three panels show operational outputs from a 40 hour sequence in a single trace of the spring scenario at $10 \%$ wind penetration. In (a) a time series of Reservoir $1 \mathrm{C}$ spill shows three individual spill events. The time series of Reservoir 1C pool elevation in (b) shows that this spill is not due to the reservoir being full (maximum elevation is $420 \mathrm{ft}$ ). In fact the reservoir is near its minimum elevation (416 ft) during these spill events. Examination of the time series of Reservoir $1 \mathrm{~F}$ pool elevation for the same hours in (c) reveals that it is also at or near its minimum elevation $(192 \mathrm{ft})$; however the outflow cannot be reduced due to environmental flow requirements. So water must be delivered from upstream to maintain the required minimum flow and prevent the reservoir from being emptied. This forces the spill at Reservoir $1 \mathrm{C}$, the third reservoir upstream from $1 \mathrm{~F}$, to send sufficient water through the system to refill Reservoir $1 \mathrm{~F}$ and maintain the environmental flows. 

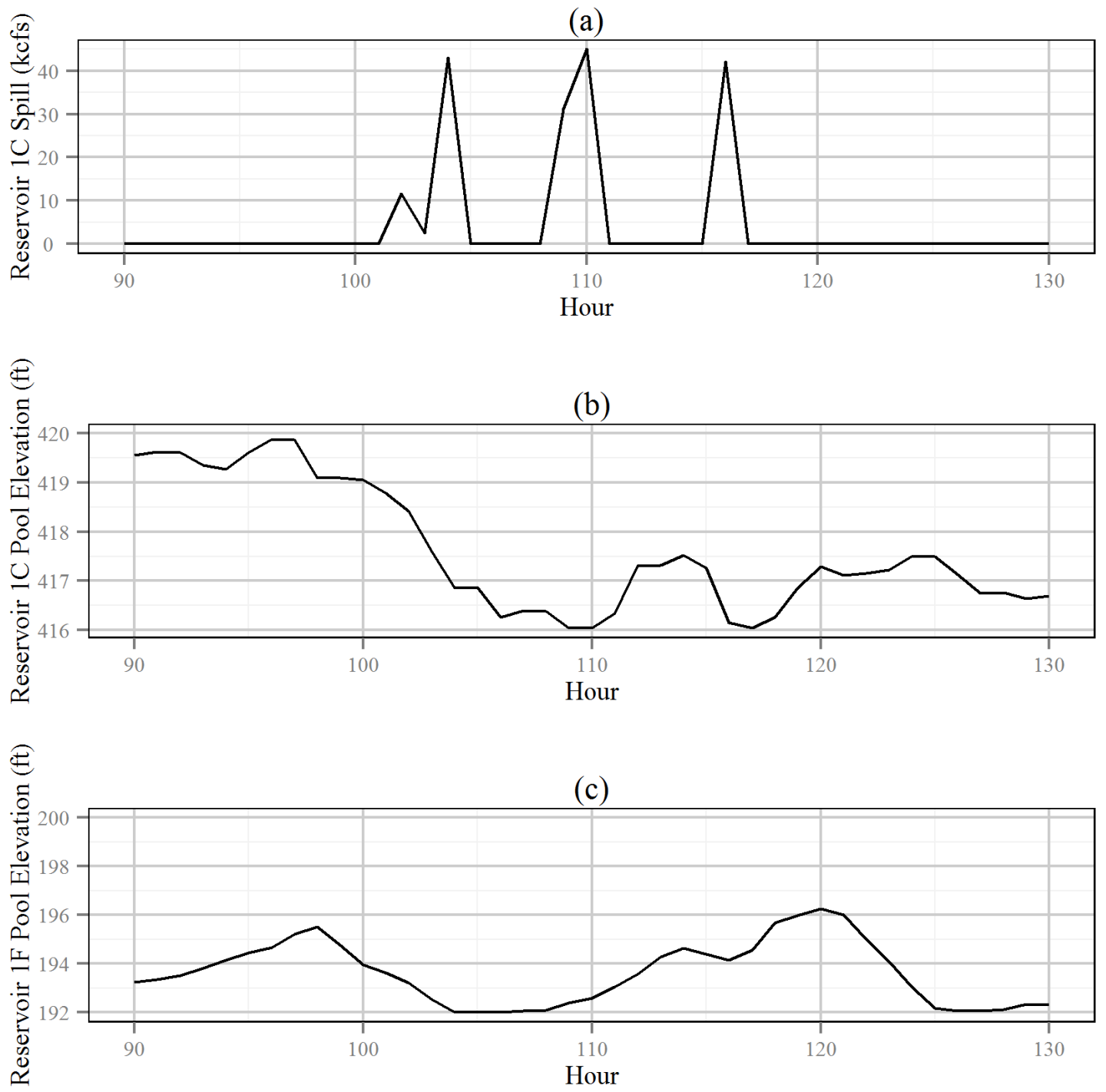

Figure C.4: Time series plot illustrating flow requirements at Reservoir $1 \mathrm{~F}$ forcing operations upstream in the system: (a) Reservoir 1C spill, (b) Reservoir 1C pool elevation, (c) Reservoir 1F pool elevation in the spring scenario at $10 \%$ wind penetration 


\section{C.3 Constraint Violations}

A summary of observed constraint violations from the spring, summer and fall base cases with full policy is shown in Figure C.5. Plots show averages for ten traces. For the spring and fall scenarios, the main non-power constraint that is violated is the minimum environmental flow requirement at Reservoir $1 \mathrm{~F}$. This constraint is violated an average of two hours out of the one-week scenario for both the spring and fall $30 \%$ case. Individual traces for the fall scenario had as many as four violations. For the summer high flow scenario, the spill limit for TDGs is the non-power constraint that is violated, with as many as five hours with excessive spill at $20 \%$ penetration. This increases to an average of nearly 30 hours at $30 \%$ wind. With high flows and high winds, this is the controlling constraint in the system. (Similar spill violations are observed at the remaining three reservoirs that are not shown here.) The $40 \%$ wind case also produces excessive spill in the spring scenario. In the summer scenario there are also deficiencies in regulation down capacity. In the high flow conditions, the system cannot reduce generation to provide regulation down without further violating the higher priority spill limits for TDGs, and so the regulation down requirement is violated.

Figure C.6 shows the violations of the load constraint in terms of (a) hours of violation and (b) the total amount of energy deficiency. The spring and fall scenarios both show increased hours with unmet load as installed wind increases. The number of violations actually decreases for the highest penetrations because the system is carrying more reserves, but when evaluated in terms of energy it indicates that the individual violations are more severe at high penetrations. When the same scenarios are run with no forecast error for the wind (but including the predictable wind variability), there are no unmet load violations. This indicates that the forecast error is the source

of the unmet load. At certain time steps when the realized wind generation is significantly less than the forecast, the hydropower system is unable to make up the entire difference. This results in the unmet load violations. 


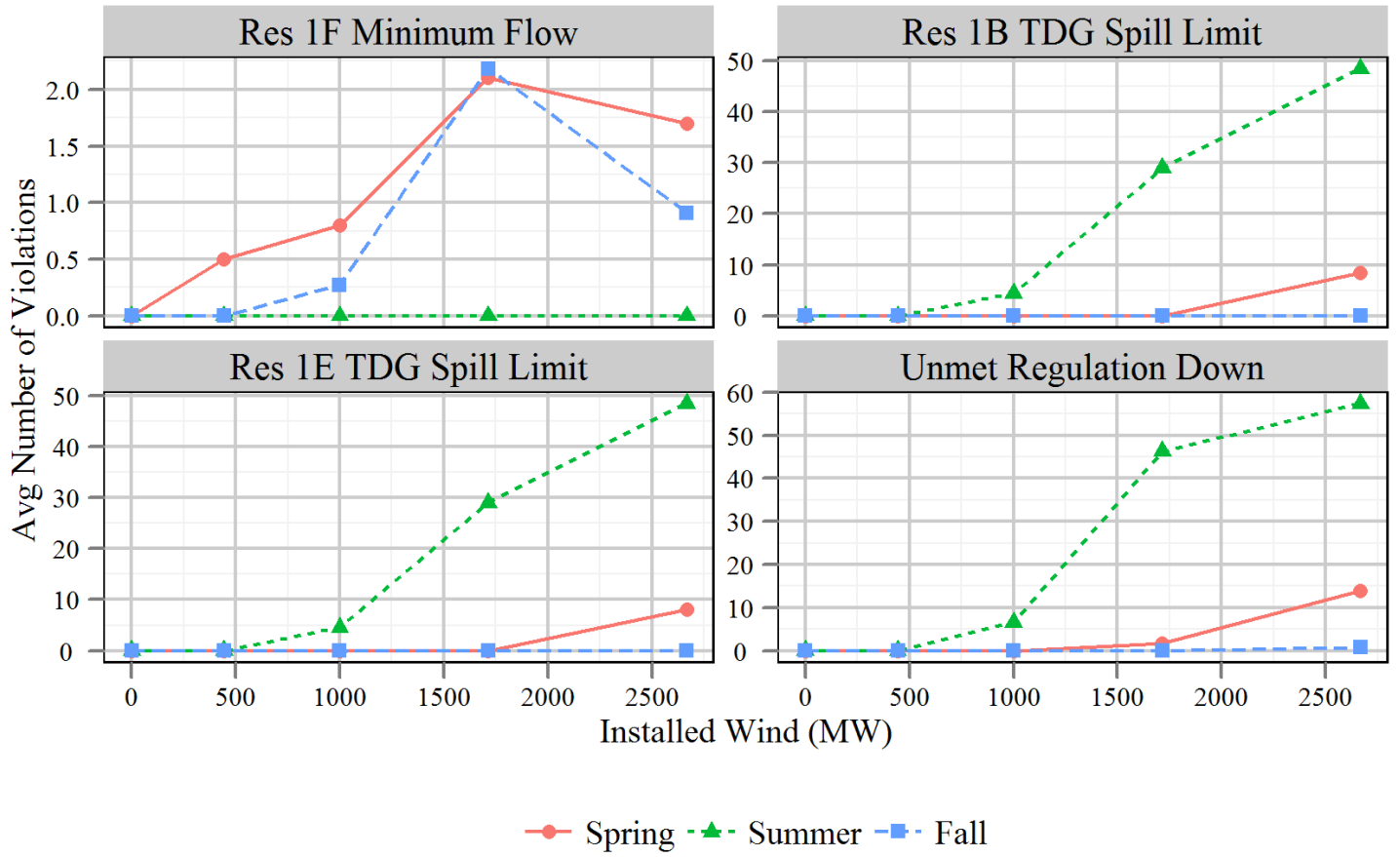

Figure C.5: Summary of constraint violations for base scenarios

(a)

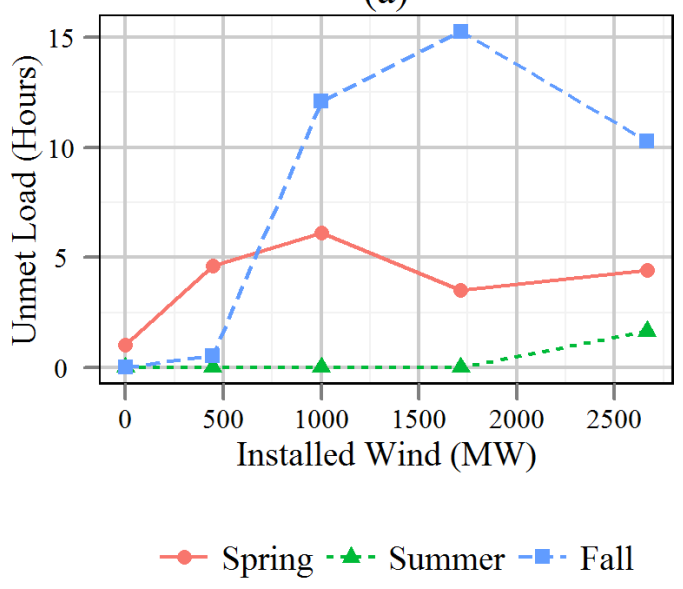

(b)

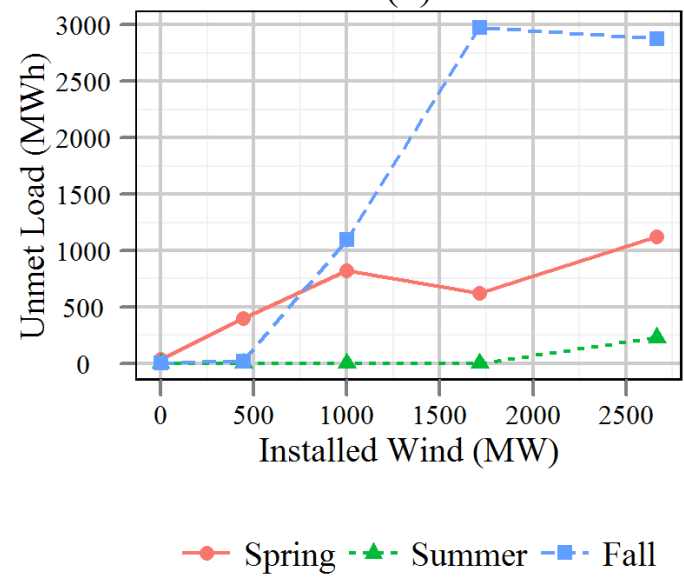

Figure C.6: Unmet load violations for base scenarios in terms of (a) hours of violations and (b) energy deficiency 


\section{C.4 Effects of Wind Forecast Error, Variability and Energy}

The effects of adding wind generation to the power system are divided into three components: energy, variability and forecast error. The energy component is generally a positive contribution. Variability and forecast error are generally negative contributions. The effects of each of these three components isolated from one another are shown in Figure C.7 for the spring scenario. (Details about how these quantities are calculated are given in Section 2.4.4.3.) Note that while the longterm average effects of forecast error and variability are assumed to be negative, in the short-term they can show positive impacts. This is observed for the isolated net value of variability in (c) and (d), which has a positive value for the $10 \%$ and $20 \%$ penetration cases. This is due to the variability of the wind generation roughly matching peak pricing.

Additional analysis examines whether the effects of wind generation forecast error on system value and spill are due to the forecast error itself or the additional load following requirement associated with forecast error. For the No Error case in Figure C.7 the load following requirement is reduced to account for the improved net load forecast when wind forecast error is removed (the

$\epsilon_{t}^{W}$ term in equation 2.85 is zero). The same scenario is run with no wind forecast error but keeping the same load following requirement from the Base case with forecast error. The results in Figure C.8 indicate that the impacts on system value and spill generally come from the additional load following requirement associated with forecast error and not the forecast error itself. The case with no forecast error but the full load following requirement shows nearly identical results to the Base case with forecast error. The same is not true, however, when considering constraint violations. Figure C.9 shows that even with the full load following requirement, the No Error case tends to have fewer violations than than the Base case with error. This suggests that the constraint violations are due to the forecast error itself, operational difficulties when the actual conditions deviate from the forecast, and not the additional reserve requirement. This also suggests that while the reserve capacity helps in meeting power constraints, it does not necessarily prevent violations of non-power constraints. 
(a)

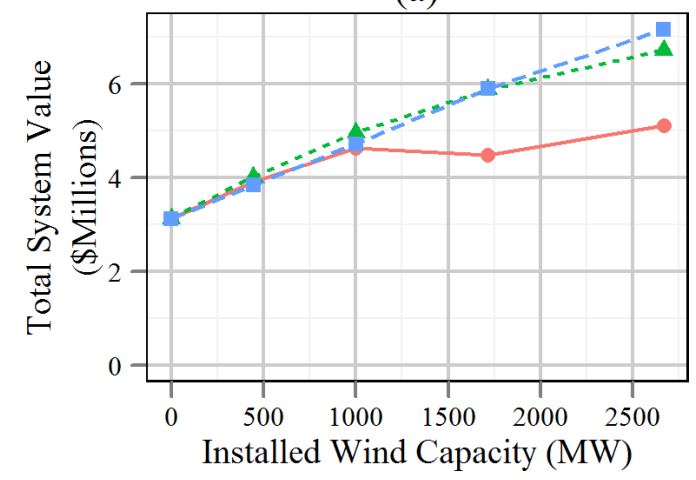

$\rightarrow-$ Base - - No Error -E- No Variability

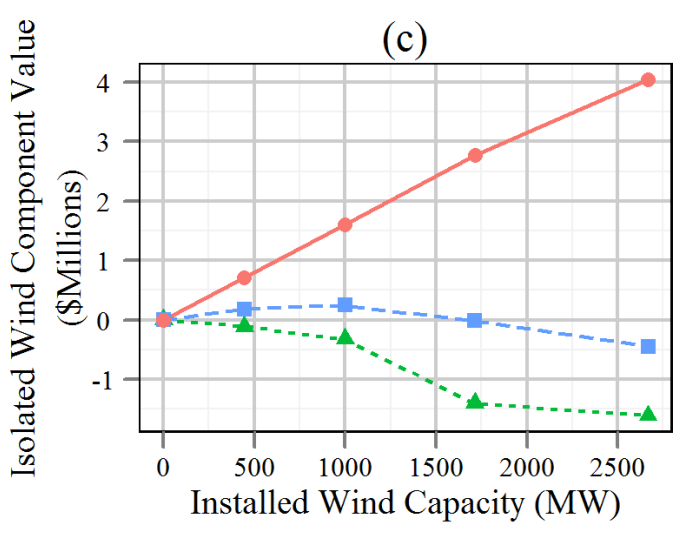

$\rightarrow-$ Energy - Error - - Variability (b)

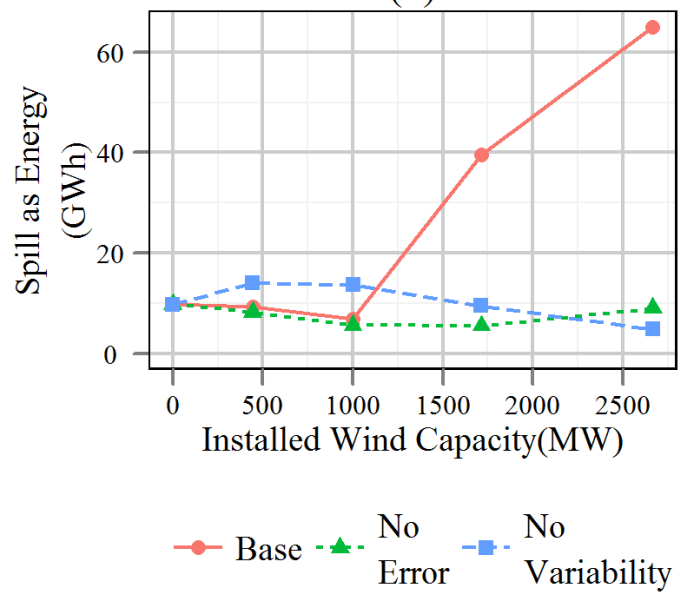

(d)

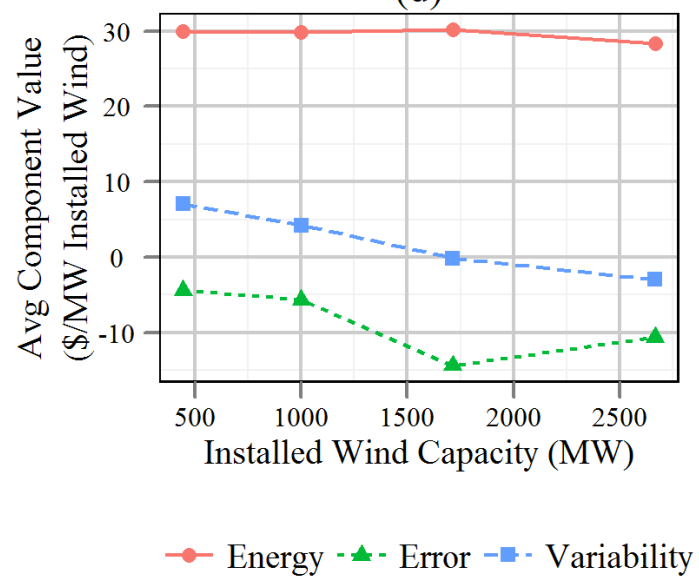

Figure C.7: Spring scenario effects of wind forecast error, variability and energy on (a) Total System Value and (b) Spill as Energy with (c) the isolated value of each component and (d) the isolated average value per MW of installed wind 


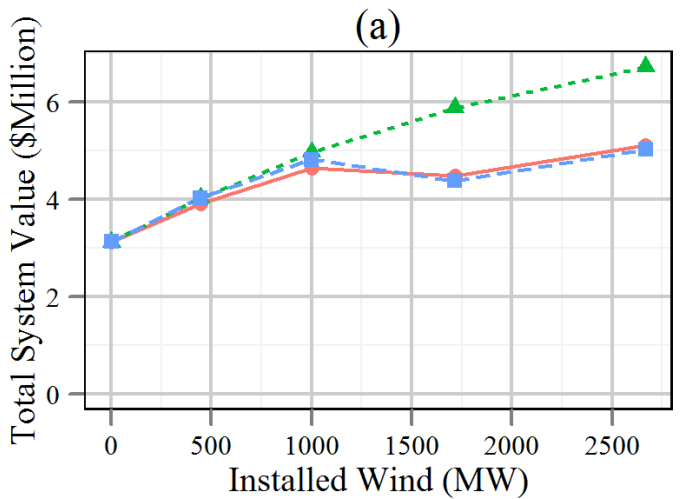

$\rightarrow$ Base $-=\begin{array}{ll}\text { No Error } & \text { No Error } \\ \text { Reduced LF }\end{array} \stackrel{-}{\text { Full LF }}$ (b)

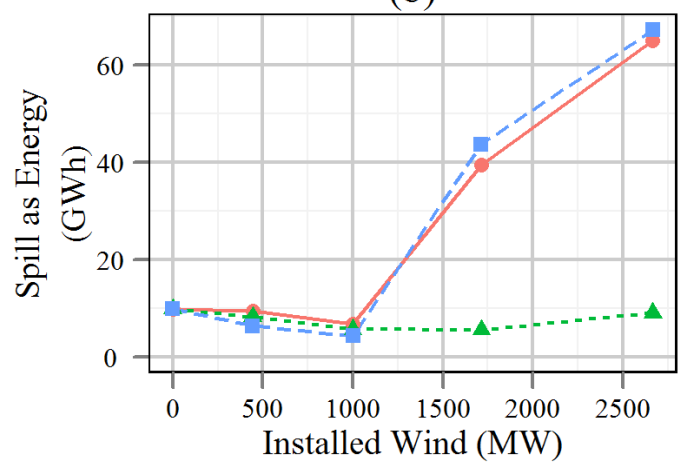

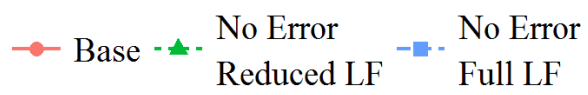

Figure C.8: Results from the spring scenario No Wind Forecast Error cases with full load following requirement and reduced load following requirement compared to the Base case showing effects on (a) Total System Value and (b) Spill (converted to equivalent energy)

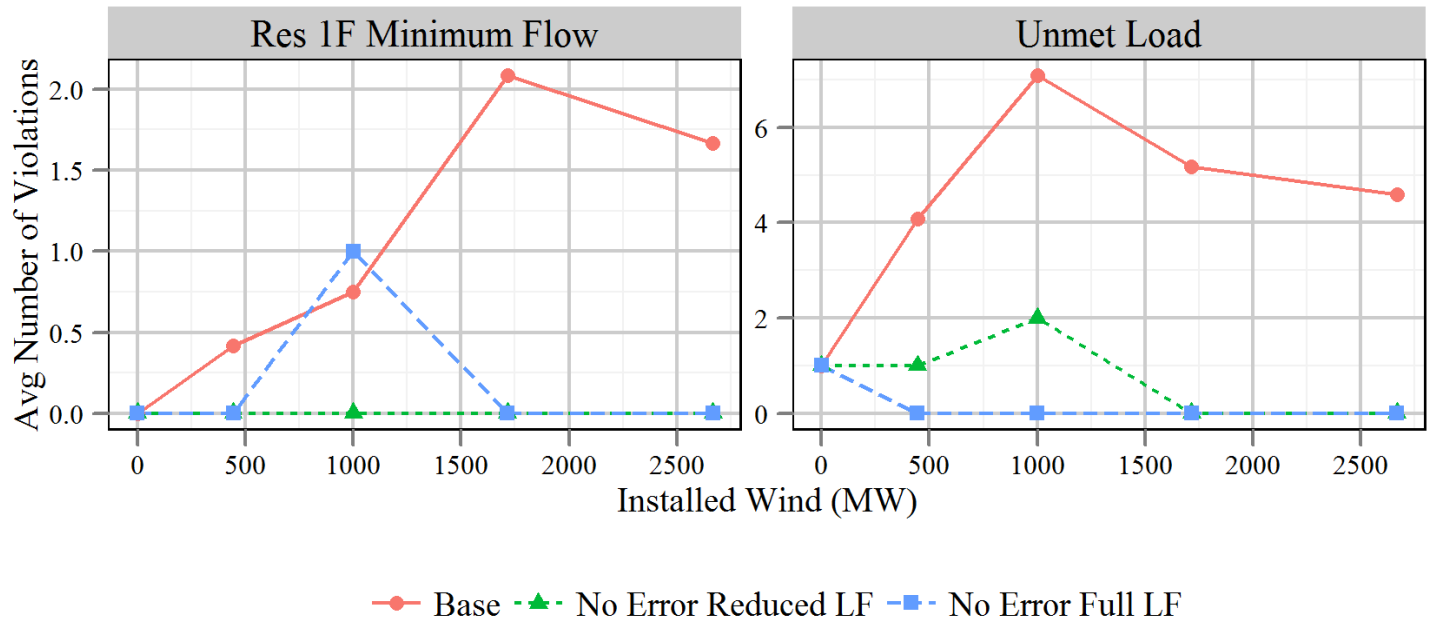

Figure C.9: Constraint violations from the spring scenario No Wind Forecast Error cases with full and reduced load following requirement compared to the Base Case 
Figures C.10 and C.11 show the effects of wind error, variability and energy for the summer and fall scenarios respectively. In Figure C.10(d) for the summer scenario, it is observed that additional wind energy has a decreasing average value even when the negative components of forecast error and variability are removed. This is due to the excess of energy already in the system, and thus additional generation added must be sold at lower marginal prices. Variability and forecast error both have negative values at all penetration levels for the summer scenario. In Figure C.11(d) for the fall scenario, the variability component actually has a positive value at $10 \%$ penetration. This is due to the timing of the wind variability matching up with peak prices. At higher penetrations, the additional reserve requirements associated with the variability outweigh this positive impact and the average variability value is negative, as would be expected. In the fall case, which is more energy limited, the value of the energy component does not decrease significantly at high penetrations. The additional wind energy is still benefitting the system. 
(a)

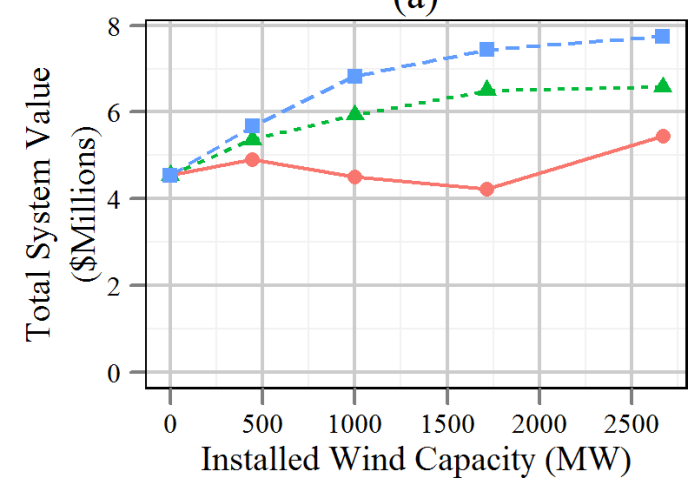

$\rightarrow-$ Base - - No Error -E- No Variability

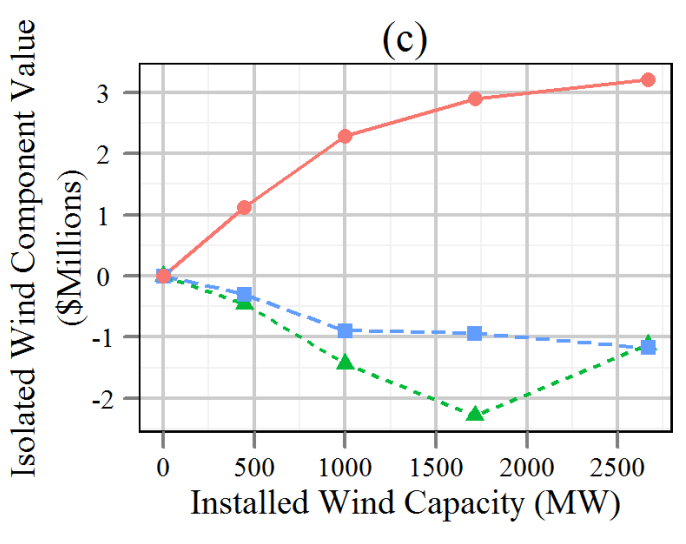

$\rightarrow-$ Energy - - Error - - Variability (b)

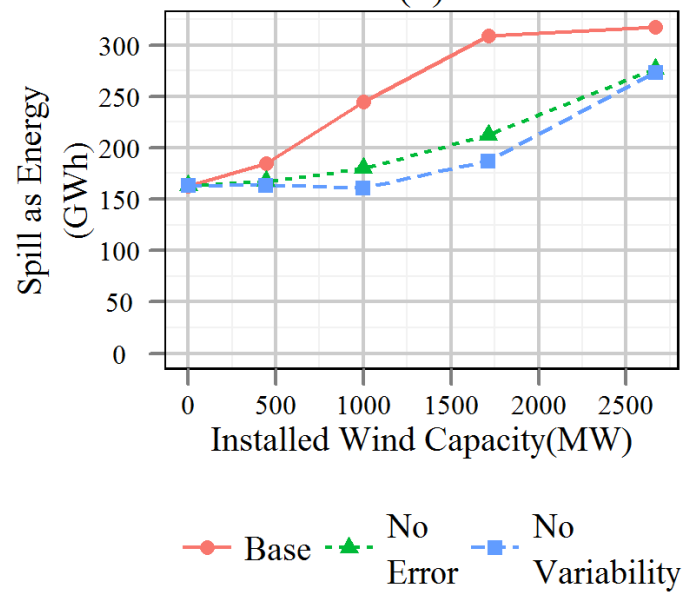

(d)

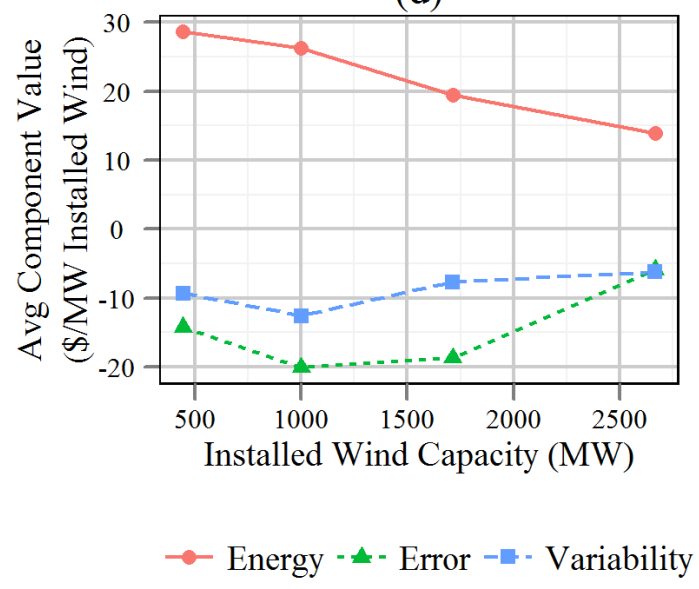

Figure C.10: Summer scenario effects of wind forecast error, variability and energy on (a) Total System Value and (b) Spill as Energy with (c) the isolated value of each component and (d) the isolated average value per MW of installed wind 
(a)

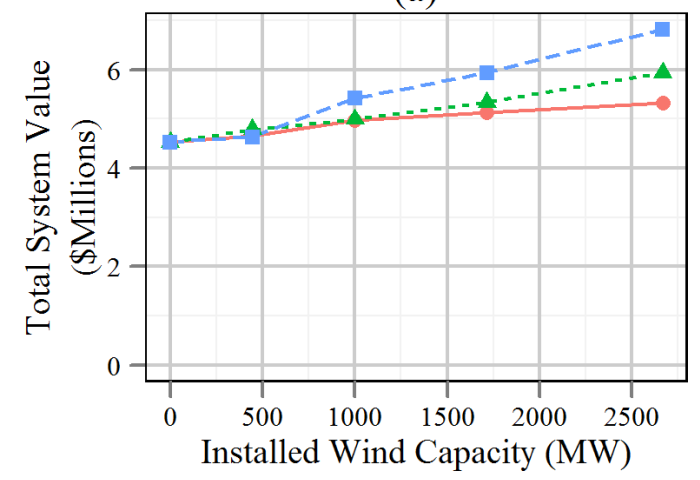

$\rightarrow-$ Base - No Error - - No Variability

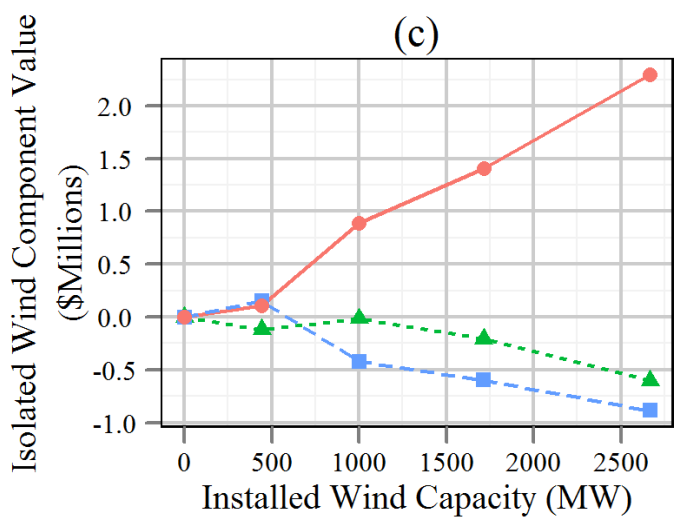

$\rightarrow-$ Energy - - Error - - Variability (b)

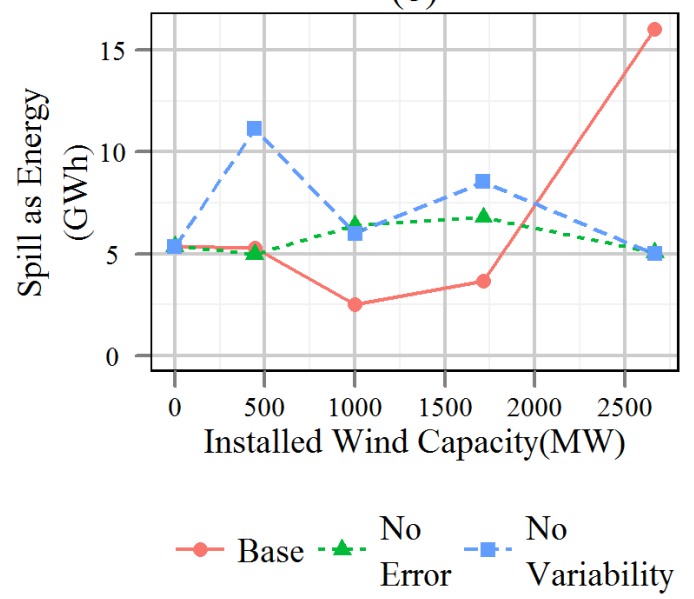

(d)

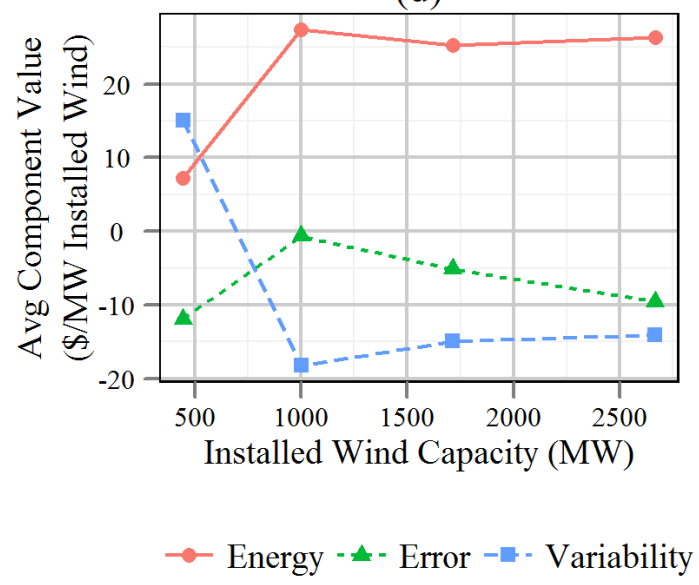

Figure C.11: Fall scenario effects of wind forecast error, variability and energy on (a) Total System Value and (b) Spill as Energy with (c) the isolated value of each component and (d) the isolated average value per MW of installed wind 


\section{C.5 Effects of Reserve Requirements}

Figure C.12 compares the load following requirement with increasing installed wind capacity with the average realized generation for the spring scenario. The graph shows that the load following reserve requirement increases at a rate greater than the actual average realized generation. It is because the reserve requirement increases at a faster rate that added wind generation is observed to have a negative value in some high penetration scenarios. While increased wind penetration adds energy to the system, at high penetrations this can be outweighed by the reduction in energy from the hydropower system due to the capacity that must be maintained as load following reserve.

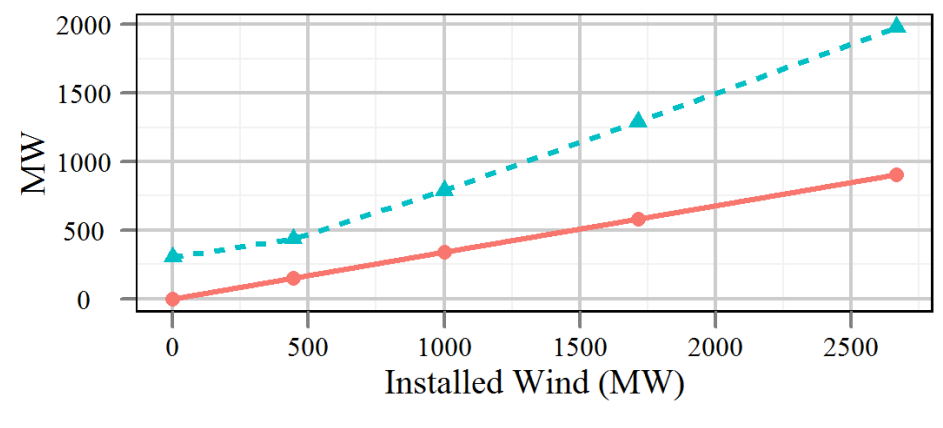

$\because$ Avg Wind Gen =^ = Load Following Requirement

Figure C.12: Load following reserve requirement vs. installed wind capacity compared to the average wind generation vs. installed wind capacity

Section 4.5.3 discusses how reducing the load following reserve requirment increases total system value at $30 \%$ wind penetration (see Figure 4.10). Figure C.13 shows that reducing the contingency reserve requirement from the baseline of $500 \mathrm{MW}$ to $250 \mathrm{MW}$ has a similar effect as reducing the load following reserve requirement. It frees up additional capacity that can be used for generation. Raising the reserve requirement to $1000 \mathrm{MW}$ has the expected opposite effect, limiting the value of added wind generation even at lower penetrations. Actual allocation of contingency reserves would be dependent on the nature of the generating resources within the BA. 


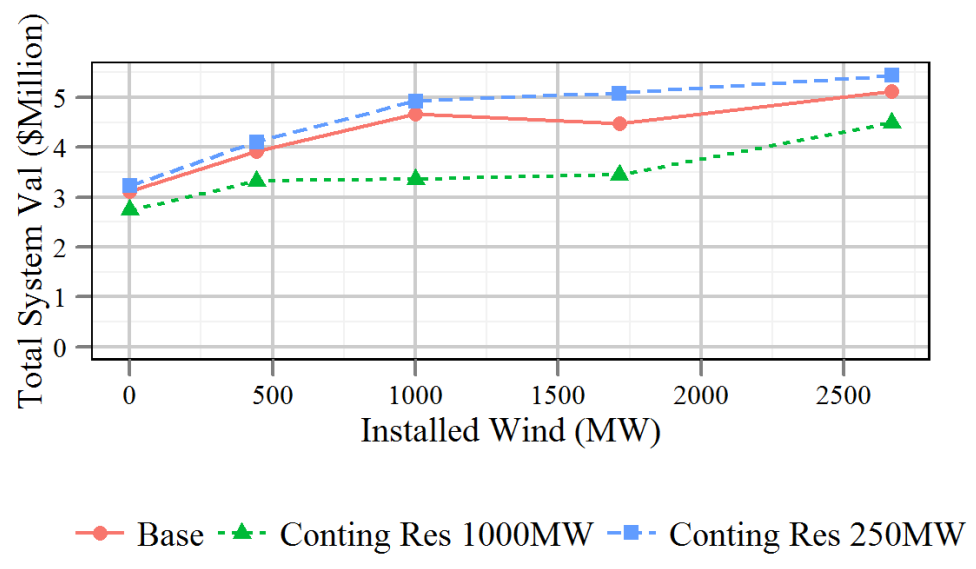

Figure C.13: Senstivity of system total value to three levels of contingency reserve requirements (spring scenario) 


\section{C.6 Summer Scenario with Spring Prices}

Figure C.14 shows results from the summer Base case scenario and the same scenario run with day-ahead energy market prices from the spring scenario to evaluate whether changes in system behavior between the spring and summer scenarios are driven more by differences in hydrologic and wind conditions or more by price differences. All other inputs are the same as for the base summer scenario. Model outputs from the scenarios indicate that there is little difference in system operations with the two price cases, only a shift of capacity away from the ancillary services market to the energy market when the energy market prices are higher. The differences in total system value are primarily due to the difference in ending energy in storage value which is based on the energy prices. The physical ending storage is shown to be the same in both cases. Total hydro generation and spill are nearly identical between the two cases. This reveals that the changes in system behavior in the summer scenario, as they differ from the spring scenario, are primarily due to the hydrologic and wind conditions and not the energy prices. 

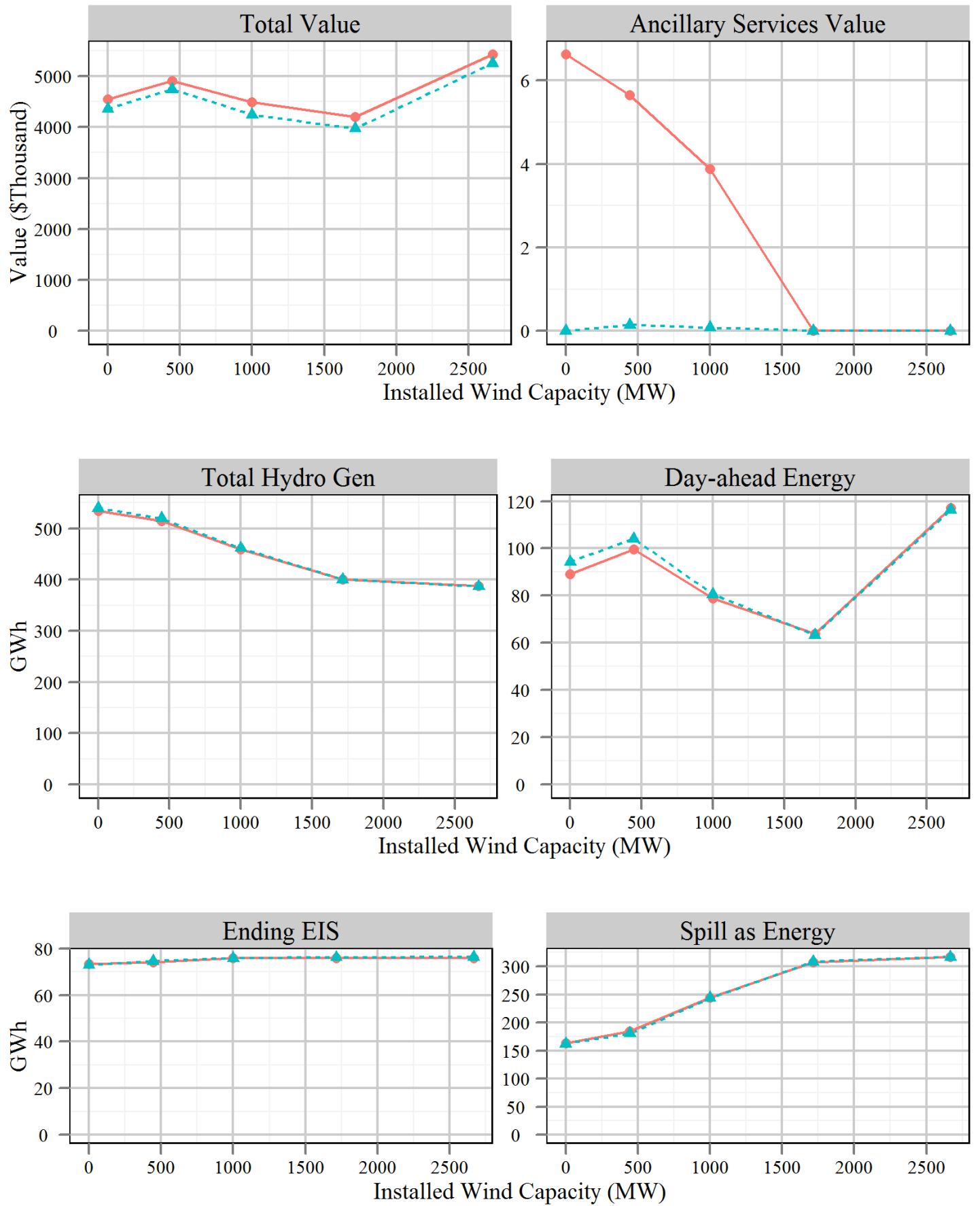

$\multimap$ Base Summer - $\_$Summer Hydrology-Spring Prices

Figure C.14: Results from the summer scenario with energy prices from the spring scenario 


\section{C.7 Effects of Load Levels and Transmission Limits}

Figure C.15 shows the expected outcome that the total system value (a) is higher when the local load is lower (note that energy to meet local load is not given an explicit economic value in the model). More capacity is available to sell in the market either as energy or ancillary services. The net contribution of wind, however, shown by its average value per installed capacity (b) is greater when the local load is higher and the additional generation is more necessary. When the local load is low, more energy is sold from the hydropower system alone before adding wind generation; therefore the added energy from wind is sold at a lower marginal price resulting in a lower net value. The opposite is true in the high load case. The added wind energy is sold at a higher marginal price. $^{1}$ These results suggest there can be a large difference in the value of added wind generation in different systems depending on how much energy they are already selling in the market and the characteristics of the load they must meet.
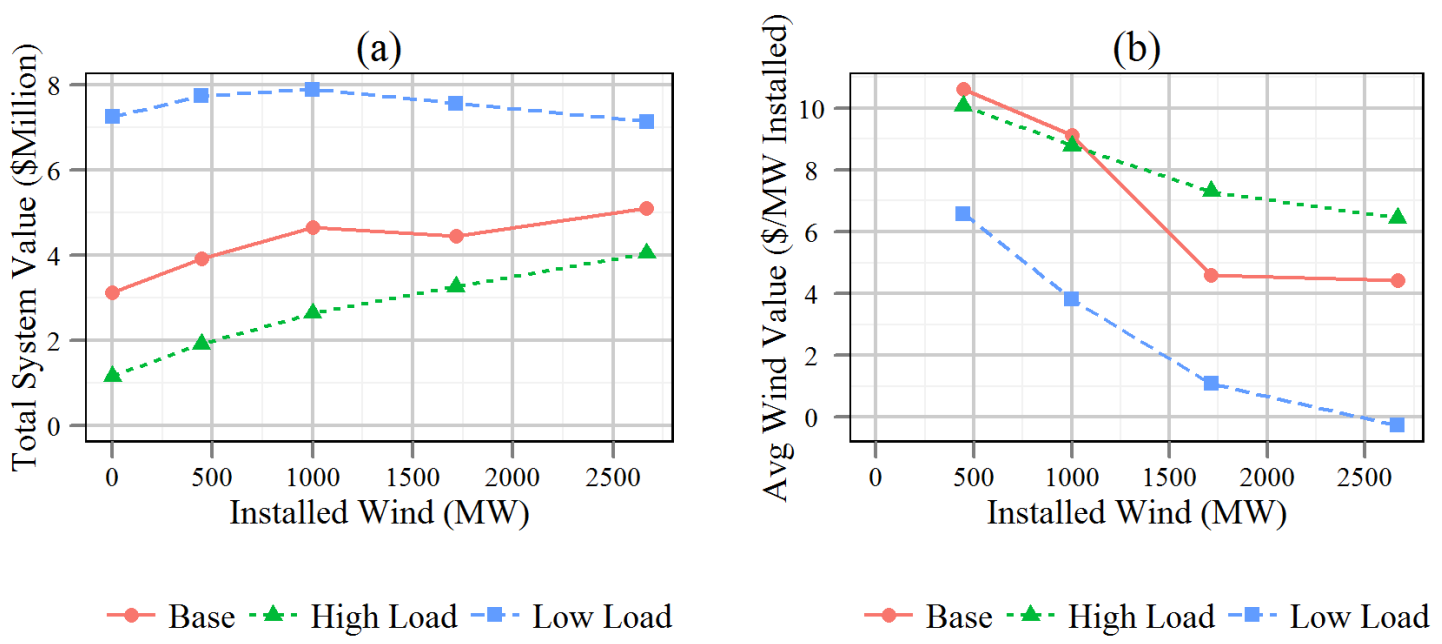

Figure C.15: Spring scenarion load level effects on (a) Total System Value and (b) Average Wind Value

\footnotetext{
${ }^{1}$ It should be clarified here that we are not making a statement about which generating source has priority and should receive a higher marginal price. The energy in our model is sold as a single quantity without distinction of the generating source. The use of terminology that suggests one generating source sells into the market first is only for the purpose of explaining the results between the different load level scenarios.
} 
When alternative transmission limits are combined with the load level cases (Figure C.16) it is seen that increasing the transmission capacity from $1500 \mathrm{MW}$ to $2500 \mathrm{MW}$ has no effect for the Base case. Transmission is not a constraining factor in the Base case. The added transmission only has value when there is excess energy in the system. For the Low Load case the added transmission provides added value at high wind penetrations in the spring scenario (a). The added transmission allows more energy to be sold into the market as more wind generation is added. In the summer low load scenario, the added value from increased transmission is only observed at low penetrations ${ }^{2}$ (b). Even at low penetrations there is excess energy in the system due to the high flows, and the additional transmission allows for more of this energy to be sold into the market. At high wind penetrations the reserve requirements become the constraining factor, not transmission, and thus there is no added value from increased transmission. Results from the load and transmission combinations suggest that it is not the sensitivity to a single parameter in isolation that is significant but rather a combination of factors in the system. Here there is no sensitivity to added transmission for the Base case loads. There is only a sensitivity to transmission limits when other factors change as well, such as load, hydrologic conditions or wind.

\footnotetext{
${ }^{2}$ Runs for the summer $40 \%$ wind case with low load and a 1500 MW transmission limit encountered infeasibility issues when trying to accomodate high levels of wind and did not run to completion. For this reason, there is no data point at $40 \%$ wind in the Low Load 1500 case.
} 

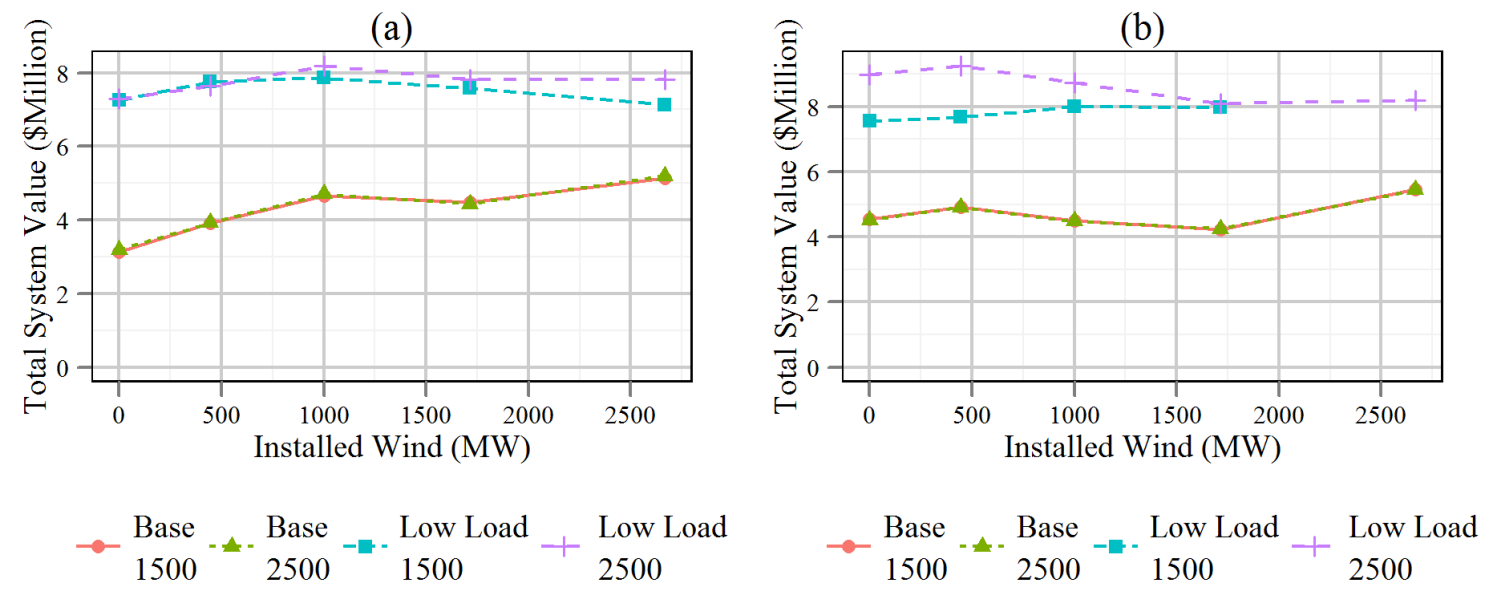

Figure C.16: Transmission and load combinations for the (a) spring and (b) summer scenarios and their effects on Total System Value 


\section{C.8 Market Depth Effects}

Market depth influences total system value. In the Base case, the day-ahead energy market clearing price is assumed to decrease $1 \%$ with each additional $50 \mathrm{MWh}$ of energy sold into the market. The Low Market Depth case increases this to 2\%. For the Unlimited Market Depth case, the price at a given hour does not change regardless of the amount of energy. The total system value changes with market depth as expected as seen in Figure C.17(a), but the total hydro generation (b) is nearly identical for all three cases suggesting there is little change in operational behavior and the change in total value is simply due to the change in prices. There is an observed shift in capacity away from ancillary services to energy when energy prices are higher in the Unlimited Depth case and the opposite shift toward ancillary services when the energy prices are lower (c).
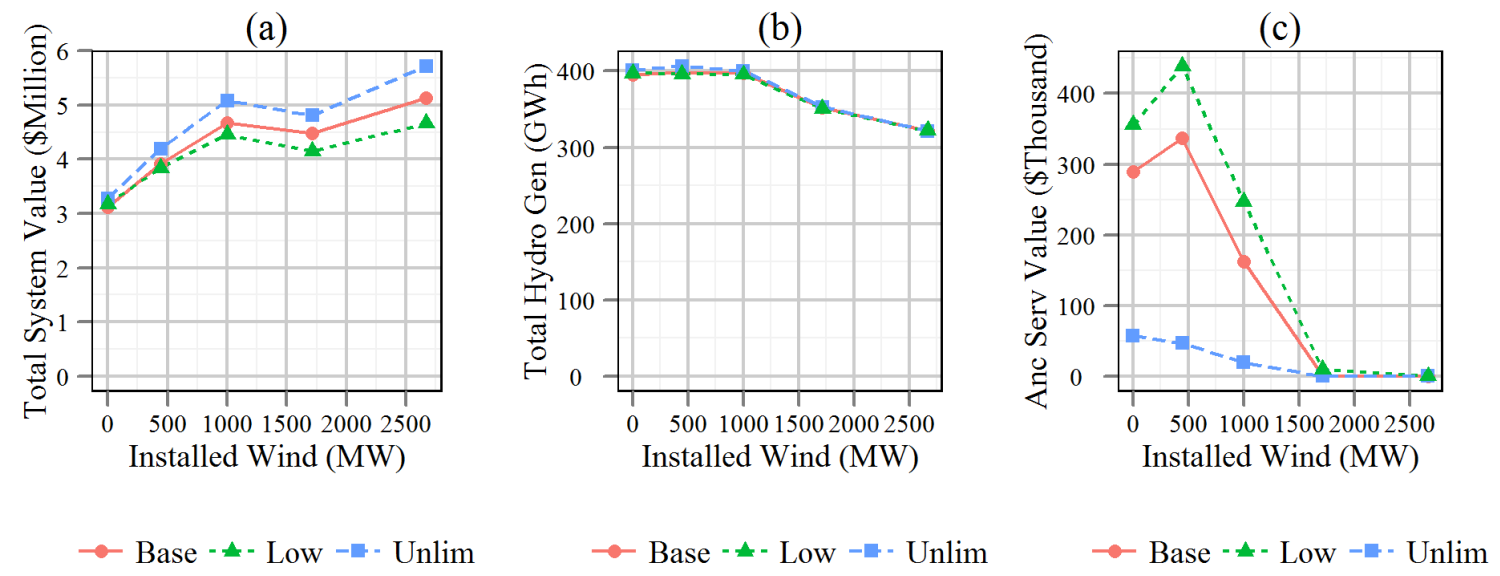

Figure C.17: Spring scenario market depth effects on (a) Total System Value, (b) Hydro Generation and (c) Ancillary Services Value

\section{C.9 Effects of Wind Timing and Correlation with Prices}

A description of the impacts on the integrated system when the timing of wind generation is shifted is given in Section 4.5.3. The wind in this system is generally in phase with peak load 
and price patterns. Figure C.18 shows that shifting the timing of wind generation back 6,12 and 18 hours reduces the total system value (a) compared to the Base case even though there is little change in total hydro generation (b). The reduction in value is due to the added wind generation no longer matching peak pricing when the timing is shifted.
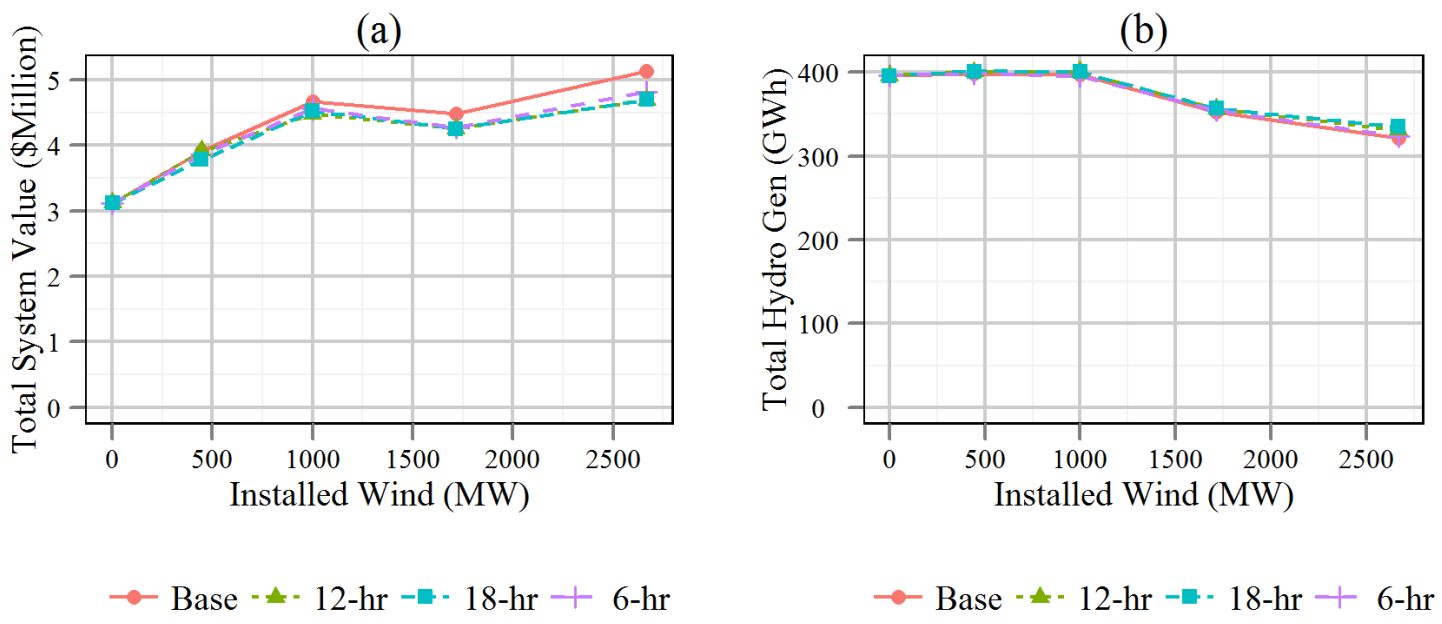

Figure C.18: Effects of shifting wind timing on (a) Total System Value and (b) Total Hydro Generation for the spring scenario 